\title{
The importance of pore throats in controlling the permeability of magmatic foams
}

DOI:

10.1007/s00445-019-1311-z

\section{Document Version}

Accepted author manuscript

Link to publication record in Manchester Research Explorer

\section{Citation for published version (APA):}

Baker, D., Brun, F., Mancini, L., Fife, J. L., LaRue, A., O'Shaughnessy, C., Hill, R. J., \& Polacci, M. (2019). The importance of pore throats in controlling the permeability of magmatic foams. Bulletin of Volcanology. https://doi.org/10.1007/s00445-019-1311-z

\section{Published in:}

Bulletin of Volcanology

\section{Citing this paper}

Please note that where the full-text provided on Manchester Research Explorer is the Author Accepted Manuscript or Proof version this may differ from the final Published version. If citing, it is advised that you check and use the publisher's definitive version.

\section{General rights}

Copyright and moral rights for the publications made accessible in the Research Explorer are retained by the authors and/or other copyright owners and it is a condition of accessing publications that users recognise and abide by the legal requirements associated with these rights.

\section{Takedown policy}

If you believe that this document breaches copyright please refer to the University of Manchester's Takedown Procedures [http://man.ac.uk/04Y6Bo] or contact uml.scholarlycommunications@manchester.ac.uk providing relevant details, so we can investigate your claim.

\section{OPEN ACCESS}




\section{Bulletin of Volcanology \\ The importance of pore throats in controlling the permeability of magmatic foams --Manuscript Draft--}

\begin{tabular}{|c|c|c|}
\hline Manuscript Number: & \multicolumn{2}{|l|}{ BUVO-D-18-00145R1 } \\
\hline Article Type: & \multicolumn{2}{|l|}{ Research Article } \\
\hline Corresponding Author: & \multicolumn{2}{|l|}{$\begin{array}{l}\text { Don Baker } \\
\text { McGill University } \\
\text { Montreal, Quebec CANADA }\end{array}$} \\
\hline \multicolumn{3}{|c|}{$\begin{array}{l}\text { Corresponding Author Secondary } \\
\text { Information: }\end{array}$} \\
\hline \multirow[t]{7}{*}{ Order of Authors: } & \multicolumn{2}{|l|}{ Don Baker } \\
\hline & \multicolumn{2}{|l|}{ Francesco Brun } \\
\hline & \multicolumn{2}{|l|}{ Lucia Mancini } \\
\hline & \multicolumn{2}{|l|}{ Alexandra LaRue } \\
\hline & \multicolumn{2}{|l|}{ Cedrick O'Shaughnessy } \\
\hline & \multicolumn{2}{|l|}{ Reghan J. Hill } \\
\hline & \multicolumn{2}{|l|}{ Margherita Polacci } \\
\hline Funding Information: & $\begin{array}{l}\text { Natural Sciences and Engineering } \\
\text { Research Council of Canada }\end{array}$ & professor Don Baker \\
\hline Abstract: & \multicolumn{2}{|c|}{ 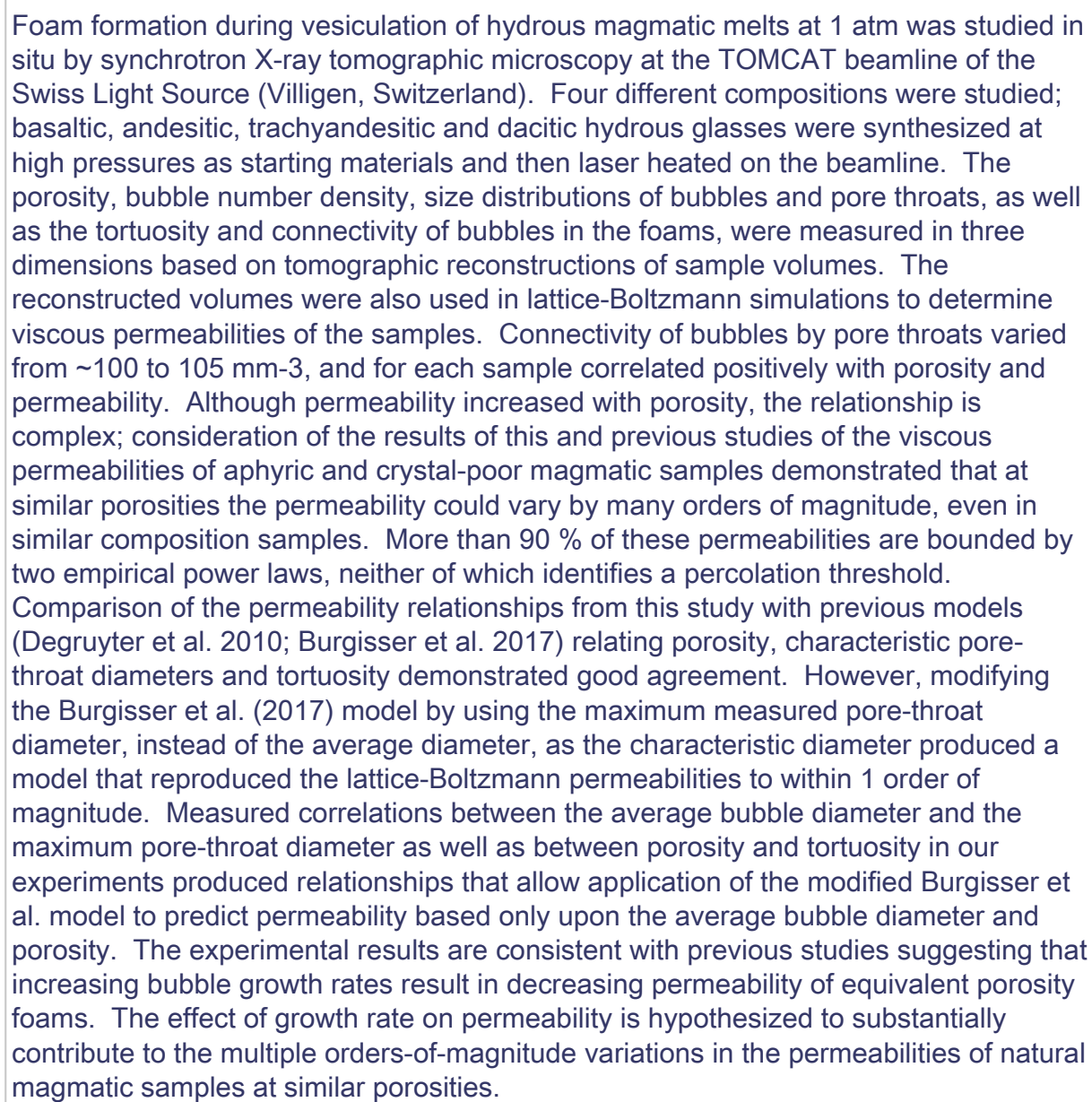 } \\
\hline
\end{tabular}


Please note that in the attached file the Editor's and the reviewers' comments are in italics, quotations from the previous version of the manuscript are in bold, and our responses are in regular text; this file is linked to the submission package but does not print with it. The locations of changes in the revised text are highlighted in the attached file, and the line numbers refer to the version of the manuscript where the changes have been made, but not accepted, so that it is easier to directly compare the previous version of the manuscript to the revised one.

Editor's and reviewers' comments:

Editor:The reviewers agree that this revised manuscript is improved relative to the original, but that there are still some important issues that need to be addressed. I am recommending moderate revisions, although I do not see the need for another round of reviews. However, I hope that you will address the comments and suggestions provided by myself and the two thoughtful reviews.

First and foremost, the manuscript requires a better discussion of how the vesiculationby-heating experiments presented here can be related to the vesiculation-bydecompression conditions that accompany most volcanic eruptions. This is not just a matter of converting an equivalence between heating rate and decompression rate, but instead requires some assessment of the effect of heating on melt viscosity and, in turn, on the kinetics of bubble growth and coalescence (pore development).

Response: The revised manuscript now has a section that implicitly discusses the differences between isobaric heating and isothermal decompression vesiculation experiments on lines $364-418$.

Another important point relates to the measurement and spatial location of maximum pore throat size. How are pore throat sizes measured?

Response: The pore throat sizes were measured using the "concept of a maximal inscribed sphere (Hildebrand and Rüegsegger 1996)" as stated in the previous version of the manuscript (I. 229-230). We have slightly expanded this explanation in the revised manuscript on lines 254-264; additional details are provided in Hildebrand and Rüegsegger. Because these samples can be modeled as random media the location of the maximum pore size does not have to be specified for the type of modeling presented in this research (e.g., lines 361-362 in the revised manuscript).

How large a volume needs to be measured to accurately assess the maximum size?

Response: This is a very important question; the maximum size of either a pore (vesicle) or pore throat was measured in the central volume of the expanding sample. For any specific sample (chosen from each sample to be most representative of the central volume of the sample) we find the maximum sized objects in it, not in the entire experimental sample. Clearly the sample needs to have multiple bubbles and pore throats within it to be measured and modeled and the maximum size of the pores and pore throats must be significantly less than the volume of the sample. We have checked this and all samples, except possibly the dacite with the highest permeability fit these requirements. The impact of the size of the sample on lattice-Boltzmann permeabilities is assessed in lines 334-355; further details can be found in Bai et al. (2010), which is cited in the text.

Isn't the spatial distribution of pore throats important?

Response: Porous media are some of the classic examples of random networks in which the pores and the connections between them (pore throats) are randomly distributed (e.g., Stauffer and Aharony 1994). Although locally the locations of pores and pore throats are important, when the sample is large enough the locations do not matter. In this study, the observations of no preferential orientation of pores or pore throats, and that the permeability varies by less than a factor of 2 between the three orthogonal directions, indicates no preferential alignment of pores or pore throats and thus supports the treatment of these samples as random networks. This argument is 
presented on lines 356-362 in the revised manuscript.

That is, is the largest pore throat is connected to the porous network via a series of smaller pore throats, then presumably it would be the smallest pore throat in the connected network that would be limiting?

Response: Flow rate control by the smallest pore diameter would occur if the pores are connected in a series. In the samples studied the connectivity is high and there are multiple interconnecting pore throats, so that transport through the pores is a parallel (rather than a sequential) process. In such a case transport appears controlled by the largest diameter pore. We have expanded this discussion in lines 876-892 in the revised manuscript:

Finally, the paper is still very long. Tightening up both the overall structure and shortening the discussion would make this much more readable. As a component of this, the terminology should be chosen carefully. As I comment on below, permeabilities that are described in the text as "determinations" or even "measurements" are actually Lattice-Boltzmann simulations from small sub-volumes. It is perfectly valid to compare these simulations with results from models (e.g., Degruyter, Burgisser). But the do refer to them as simulations and not as measurements, for the sake of clarity.

Response: We have both tightened up the overall structure and reduced the length of the manuscript, as well as changing the terminology as requested. In particular, we have removed both the discussion of Namiki and Manga model from the revised manuscript and the comments on inertial viscosity.

I. 124-127 A comment: permeability anisotropy may also be important in gas loss from volcanic conduits (e.g., Schneider et al., 2012).

Response: We have now referenced the paper by Schneider et al. (2012) on line 819, but in this contribution we are only dealing with permeability in isotropic samples.

I. 145- I infer from Fig. 2 that the sample was allowed to expand freely? This should be stated, as free vs. confined expansion is an important distinction from the perspective of bubble-bubble interaction and anisotropy generation.

Response: The samples freely expanded; this has been explicitly stated in the revised manuscript on line 183.

I. 166-176 See comments above, and of one reviewer, about changes in melt viscosity during heating, which is also relevant to the thermal gradients mentioned in I. 204.

Response: The revised manuscript now has a section that explicitly discusses the differences between isobaric heating and isothermal decompression vesiculation experiments on lines 364-418 that includes a presentation of the changes in melt viscosity and water diffusivity during bubble growth.

I. 204-225 By my calculation, it looks like all of your experiments were at a resolution of $2.89 \mu \mathrm{m} / \mathrm{pixel}$, or $24 \mu \mathrm{m} 3 / \mathrm{voxel}$ ? Is the criterion of $>2$ voxels $(>\sim 50 \mu \mathrm{m} 3)$ sufficient to image pore throats in all samples?

Response: Yes, we were able to image all pore throats whose sizes were greater than 2 voxels in the samples. However, small pore throats below the imaging resolution could not be detected.

Another question about resolution: how do the volumes used for the LB simulations compare with the mean and maximum bubble sizes?

Response: With only one exception, the highest porosity dacitic sample, the maximum bubble volumes are less than $6 \%$ of the volume used for the LB permeability simulations. This has been stated in the text on lines 467-470.

It would help to provide a slightly more detailed description of the "inflation of spheres" 
method. Inflation seems an odd word to use... from an image processing perspective, is this a dilation? It is also a bit difficult to see the difference between Figs. $1 \mathrm{f}$ and $\mathrm{g}$... can you provide a quantitative comparison as well as a visual?

Response: We chose the word "inflation" explicitly to imply that the spheres are enlarged isotropically until they touch the walls of the bubble or the pore throat. We did not use the word "dilation" because dilation can be anisotropic. We have now used the phrase "isotropic inflation" because one of the reviewers wondered if the inflation was isotropic (line 271 in the revised manuscript).

I. 264 Define the Betti number

Response: We have deleted use of the term Betti number in the revised text as one of our space-saving modifications so a definition is no longer needed.

The section on Lattice-Boltzmann modeling of permeabilities contains quite a long discussion of Bai et al. (2012); can you shorten to the most salient points?

Response: The discussion has been shortened (lines 334-355 in the revised manuscript).

Figure 2 is not particularly informative (although interesting that the sample maintains sharp edges during expansion), and it is not possible to see the details of bubble growth (e.g., I. 342). My suggestion would be to use fewer images, but to include labels in each image of sample volume and/or vesicularity. Also useful would be a diagram showing the nominal change in melt viscosity over the temperature interval of 600 $1100^{\circ} \mathrm{C}$.

Response: We have modified Figure 2 and added vesicularity of the subsample investigated, as well as estimated viscosities (calculated following Giordano et al. 2008) into it.

I. 349 What is meant by "Bubbles coalesced and typically grew to a maximum size, creating a foam of thin-walled bubbles"? Are you simply saying that heating under free expansion creates high vesicularities? I note, however, that the maximum vesicularities reported in Table 2 range from $64-84 \%$, which are not unusually high, with the lower not technically a foam.

Response: Yes, that is what we mean. We have changed our wording, but retain the use of the word foam in some instances throughout the manuscript to describe the samples.

I.356 If surface area is important, did you experiment with different resolutions of tomographic images?

Response: We did not experiment with different resolutions. We used the maximum possible resolution on the beamline in order to obtain the best images possible of the representative sample volumes.

The section on BNDs, BSDs, and PTDs could be condensed.

Response: This section has been condensed, primarily by removing the sentences describing average values in the samples.

I must be missing something... in Table 2, the superscript 1 is supposed to be for the volumes measured, but I don't see any column that reports this; the only reported volumes appear to be those used for permeability measurements.

Response: This was my mistake. I submitted an older version of Table 2 that included other mistakes. The table has been revised in the current version of the manuscript.

I. 368 I don't see the connection between continuous nucleation (during heating) and water content? 
Response: There has been some discussion in the literature concerning continuous nucleation versus a single nucleation event in the experimental literature. Because this topic is not particularly important to the study at hand we have removed this sentence to shorten the manuscript.

I. 381-383 Why present mean and standard deviation of bubble sizes when you have already stated that none of the distributions are Gaussian? The mode (or even the median) is probably more useful.

Response: The mean and standard deviation were supplied to provide the reader with a means to compare the current results with those of previous studies (see lines 378382 in previous version of the manuscript). We have now also provided the median value of the bubble and throat diameters in Table 2. And, we provide all of our measurements in the Supplementary data table so that readers can make their own calculations.

Also important are the volume-based (rather than number-based) distributions (e.g., Klug et al., 2002), as these will give a sense of the samples where a few large bubbles contribute much of the volume; this may be important for thinking about sub-sampling for permeability calculations.

Response: We certainly agree that volume-based distributions such as those used in Klug et al. (2002), are extremely useful. However, in the context of this manuscript we do not think that their inclusion would help the reader better understand the experiments, and the discussion of volume-based distributions would add unnecessary length to the manuscript.

Additionally, for both number- and volume-based distributions, I find it easier to compare samples if you put several together on a cumulative distribution plot, rather than numerous separate histograms. Cumulative distributions also circumvent problems of binning, and allow direct comparison of median values.

Response: We have now put the cumulative distributions back into Figures 3-6 because we agree that the cumulative distributions circumvent the problems of binning. We did not include a single plot with the cumulative distributions in this manuscript previously because we did not concentrate our investigation on the changes in the distributions, and a single plot containing all of the cumulative distributions is extremely "busy". Because all of the data are included in the Supplementary Materials the reader can create such a plot if they are interested.

I. 402-412 I'm a bit confused. You explicitly state that you avoided counting "anomalously" large bubbles, and then focus on the large number of small bubbles (which, by definition, are the only ones you are counting?)

Response: We have rewritten these sentences to stress that we chose representative volumes of the sample near its the center and avoided regions with anomalous bubbles that were found near sample edges (lines 516-520 in revised text).

I. 445 Your reported $\beta$ values are very high, which presumably relates to the volume normalization? I don't have an intuition for this number (or its purpose)... average coordination numbers of 4-6 are interesting and actually lower than expected theoretically for uniform spheres (which should be 12). I am a bit puzzled by the maximum number of 600 , however... is this an artefact of the processing or is it simply one very large bubble in a mesh of tiny bubbles?

Response: The high numbers come from large bubbles with a mesh of interconnected tiny bubbles. A two-dimensional example of this can be seen in Figure 1. See lines 576-578 of the revised manuscript.

1.470-487 You are mixing apples and oranges here. Relevant for your analysis are samples that have experienced only simple vesiculation histories, in the absence of abundant crystals or bubble deformation. The low percolation value from Saar and Manga, in contrast, is a sample from a basaltic lava flow that is both highly crystalline and has lost most of its original porosity. What is the rationale for extending even your 
andesite line to infinitely small porosity (which would require crack-like geometries), rather than assuming that samples with lower porosities are actually not permeable (that is, lack a connected bubble network)?

Response: As stated on lines 482-483 in the previous version of manuscript and in multiple places in the revised manuscript, the critical porosity threshold for any specific sample is unknown. This is due to the size distribution and shape of the vesicles (mentioned on lines 479-480 and more thoroughly discussed on lines 575-600 of the previous version and lines 602-612 in the revision). Because the critical porosity threshold is unknown for our samples we chose to use an empirical fit as done in some previous studies (see lines 484-485 of the previous version and lines 602-612 in the revision) and acknowledge that other studies have estimated the critical porosity and used it in their fitting (lines 485-487 in the previous version and 606-612 in the revision). In the previous version we also included a fit with a critical porosity to the andesitic data (see line 491 of the previous version) and keep that fit in the included revision (line 616).

As mentioned above, I found the discussion of Fig. 7 confusing, in that you discuss simulated porosity-permeability relations as if they were measured. Be clear about this, especially as this section follows a long section on measurements, and you don't let the reader know that you are now switching to LB simulations.

Response: We have now reworded the revised version to make clear in the discussions of Figures $7 \& 8$ that the reported permeabilities come from lattice Boltzmann simulations.

Regarding Fig. 8; again, I would suggest being more discriminating here, so that you make your comparisons only with crystal-poor samples that have experienced a simple vesiculation history (which is the more appropriate comparison for your samples).

Response: Following this suggestion we have modified Figure 8 to present only aphyric-to-crystal-poor samples. The changes in Figure 8 have no effect on the conclusions reached in this study, although the power-laws that encompass the measurements change from when both aphyric-to-crystal-poor samples and crystalrich samples are included.

Discussion - at almost 19 pages, the discussion is too long, even for someone like me who is interested in the topic!

Response: We have significantly shortened the discussion in the revised version of the manuscript and removed the discussion of the Namiki and Manga model. The discussion has been reduced to 11 pages in the revised manuscript.

I would start by shortening the section on permeability "determinations". This is an odd word, because it sounds like you measured them... I would use the word "simulated".

Response: We have shortened the section and changed our wording to make clear that our permeabilities are the product of lattice Boltzmann simulations.

I don't know the Bai et al. paper well so I won't comment on these data. However, the highly variable permeabilities at very low porosities in the Saar and Manga data set are certainly crack-dominated, as mentioned about (they are completely solidified and mostly degassed lava flow interiors). Similarly, as we have pointed our (Rust and Cashman, 2004; Wright et al., 2009; see also Wright et al., 2014), the permeability of effusive samples and/or densified (domes, flows, welded tuffs, etc.) samples is the end result of both vesiculation (sometimes in the presence of numerous crystals) and compaction, gas loss (densification) and, sometimes, later crack formation. For this reason, it does not make sense to fit them all to the same porosity-permeability law. Finally, the hard sphere percolation concept neglects the fact that bubbles become permeable either by deforming (as in tube pumice) or by coalescing; the latter requires melt film thinning and rupture, which will occur at rates that depend on melt viscosity and the pressure differential across the melt film (rate of bubble-bubble expansion). Setting out these general constraints at the beginning of the section would allow you to reduce, substantially, the amount of time spent discussing individual sample suites. 
Response: We have followed these suggestions in the revised version of the manuscript by only discussing aphyric-to-crystal-poor samples and avoided "effusive samples and/or densified samples". We also do not think that the hard-sphere percolation threshold is important, but think that the readers need to be informed of its existence so we have kept it in the revised version.

The section on model comparisons could also be shortened, as there are places that are repetitive.

Response: We have shortened the section on model comparisons.

I. 657 Define "channel circularity". To me this is a 2D parameter based on a comparison with a circular cross-section. How, then can this have the value of 10 ? What sort of geometry does this imply (if it is even physically reasonable?)

Response: Channel circularity was defined in the previous version in Equation 4 (Line 658 and in the revised version in Equation 7, line 841-844). It is defined by both the equivalent circle radius of the throat, $r$, and its major axis, I, following Degruyter et al. (2010), so it is basically a 2D parameter measuring deviations from circularity.

Note that for a major axis 4 times the equivalent circle radius the value of $X$ is 16 . Also, we now discuss the value of 10 as a functionof $2 \times$ a fitting constant of 5 .

I. 676-678 Here again you refer to simulation results as "measurements". As noted above, this would be clearer if you used the term simulation.

Response: We have made the changes to indicate the permeabilities are from latticeBoltzmann simulations.

I.682-734 This is an excessively long paragraph. L.682-692 could be omitted, as a start.

Response: We have shortened this paragraph greatly in the revised manuscript (Lines 743-774), but think we need to include the description of percolation theory in the beginning of this paragraph to help the reader better understand the discussion.

Explain how the largest pore throat can dominate permeability. As noted above, it doesn't matter how large the PT is, if it is connected to the larger network by only tiny PTs. Indeed, we know that permeability is not controlled by the largest bubble. The more common way to think about this is that the largest(most permeable) pathway dominates.

Response: Our experiments, with their high connective densities, provide evidence the the bubbles in the samples are multiply connected and that therefore they can be modeled as parallel circuits. In such a parallel circuit the transport will be dominated by the pathways of lowest resistance, the largest pore throats. (see lines 876-892 in the revised manuscript)

Figure 9c. Again, I don't know the Bai data very well but they do not show a very convincing fit (wrong shape?)

Response: We agree that the Bai et al. data do not show a convincing fit and wish that the Bai et al. data did fit the model better. However, they are some of the few data to which we can compare our model, and we think it important to present the comparison between the model and the Bai et al. (2010) data. In the previous version of the manuscript we made the quantitative comparison between the model and data and commented upon its limitations (Lines 718-728 in the previous version and lines 914923 in the revised version). We specifically stated that the model accuracy was degraded when using equation 6 to estimate the permeability and demonstrated that the predictions were only accurate to approximately 1 order of magnitude.

I.729 The caveats are good but belong earlier! 
Response: We moved the caveats to an earlier position in the discussion (lines 867875 in the revised manuscript).

I. 748 I would expect a relation between bubble growth rate and pore throat size simply on the basis of the relation between bubble growth rate and $\Delta \mathrm{P}$ (e.g., Klug and Cashman 1996).

Response: That certainly makes sense, but unfortunately our results are too limited to test this hypothesis.

Omit the discussion of inertial permeability, and reference Zhou et al. (2019) instead.

Response: This has now been done in the Introduction where we also discuss Polacci et al. (2014) who found the same relationship as Zhou et al. (2019). Please see lines 135-139 in the revised manuscript.

Again, it would make more sense to use a porosity-permeability model based only on expanding (vesiculating) crystal-poor samples in the Namiki and Manga model, rather than muddying the waters with all measured data. Additionally, with the very slow rates of magma ascent used, both two-phase flow (in low viscosity melts) and loss of gas to walls rocks (e.g., Schneider et al.) could be important.

Response: We have now dropped this discussion. Note that our bounding values for the crystal-free to crystal-poor permeability-porosity data are not significantly different from found when crystal-bearing samples are also used for the fit.

I also have a question about the modelling, particularly with reference to Fig. 10 . The patterns of gas flow shown here, with peak Vgas $/ V$ melt for basalt at $7 \mathrm{~km}$ depth and $12 \mathrm{~km}$ for rhyolite, do not make sense with respect to the stated range of water solubilities (70 MPa and $180 \mathrm{MPa}$; which are equivalent to 2.8 and $7.2 \mathrm{~km}$ for a generously low crustal density of $2500 \mathrm{~kg} / \mathrm{m} 3$ ). Is this all a function of reduced density in the conduit because of the steady state assumption?

Response: Yes, the density becomes quite low because of the bubbles in the magma.

It would be helpful to include a plot of exsolved gas fraction as a function of P. Also, you might state that the form of the Vgas/Vmelt curve is a direct reflection of the assumed shape of the permeability curve, which increases by several orders of magnitude between 0.0 and 0.07 volume fraction porosity.

As you note, however, if this were actually the case, then we'd never see bubbly magma reach the surface. This does not necessarily mean, however, that your average permeability curve is correct. A more plausible explanation for the abundance of bubbly samples of all compositions is the existence of a porosity threshold for the onset of permeable gas loss.

Response: The Vgas/Vmelt curve is a direct reflection of the assumed permeability curve shape, which is why only the highest values of permeability cross the threshold for potential gas loss. What is interesting is that irrespective of the exact type of porosity/permeability relationship, the threshold is crossed only for the most permeable magmas and this permeability threshold is approximately $1013 \mathrm{~m} 2$ (see Figs. 10 and 8 in previously submitted version of the manuscript). The Namiki and Manga model is very interesting and predicts very interesting magma behavior that is far beyond the scope of the current manuscript. Therefore, because we cannot fully discuss the model under different porosity-permeability conditions without making the manuscript substantially longer (e.g., comparing magmas with and without porosity thresholds for permeable behavior), with regret that we have dropped the Namiki and Manga model from the revised manuscript.

Klug, C., Cashman, K.V, Bacon, C.R. (2002) Structure and physical characteristics of pumice from the climactic eruption of Mt. Mazama (Crater Lake), Oregon. Bulletin of Volcanology 64:486-501.

Schneider, A., Rempel, A.W., and Cashman, K.V. (2012) Conduit degassing and 
thermal controls on eruption styles at Mount St. Helens. Earth and Planetary Science Letters 357-358: 347-354.

Wright, H.M.N and Cashman, K.V. (2014) Compaction and gas loss in welded pyroclastic deposits: evolution of porosity and permeability in the Shevlin Park Tuff Geological Society of America Bulletin 126: 234-247. doi:10.1130/B30668.1

Reviewer \#3: Review of "The importance of pore throats in controlling the permeability of magmatic foams" by Baker and others.

This paper presents results of new incremental heating vesiculation experiments of crystal-poor volcanic samples. The samples are progressively imaged using threedimensional tomography in order to characterize the evolution of pore size, connectivity and volume through time. The authors present the results of image analyses, permeability modeling, comparison with published measurements for other volcanic samples, and apply the results to models of syn-eruptive conduit flow.

The one major shortcoming of this paper is the lack of direct connection between the experiments presented herein and their applicability to natural systems. Furthermore, although the authors repeatedly suggest that porosity is not the only control on permeability, the discussion applies a single fit (and bounding upper and lower limits) between porosity and permeability to all data from the literature. No attempt is made to separate out subgroups lumped according to hypothesized controlling factors (bubble growth rate, crystal content). As such, the application of the final model from Namiki and Manga (2008) feels disconnected from the experimental results presented herein.

Thanks for the opportunity to review, Heather Wright

Please find detailed comments below and new plots created from data in Table 2 presented in the attached excel file.

Line 75. Loss of gas can occur through porous networks or via two-phase flow. Perhaps reword this statement by saying 'gas loss' and removing the porous network portion of the sentence.

Response: We have changed the sentence following the reviewer's suggestion (Lined $82-83$ in the revised manuscript)

Line 80. This is true only when bubbles cannot move through the melt on their own. As above, two-phase flow can also prevent pressurization that causes explosions.

Response: We have slightly changed the sentence in question to: "Relatively impermeable magmas can lead to violent eruptions whereas permeable ones may not (Sparks 2003; Mueller et al. 2005, 2008)." on lines $87-88$ of the revised manuscript.

Line 89. This sentence is vague. How about: 'proposed separate power-law relationships"...

Response: In order to condense the manuscript this line was deleted from the revised version.

Line 99. Change number of pore throats to number density of bubbles?

Response: Line 98 of the previous manuscript (which is part of the same sentence as line 99) mentions the importance of bubble size distributions and we decided to keep the phrase "the number and size of pore throats" because of their importance in the understanding of the permeabilities of the studied samples".

Line 134. Is there any additional information about the chosen samples? Where is the MORB sample from? Are the Atkan andesite and dacite samples from historic 
Response: We do not have data on the exact location of MORB. The Atka andesite and dacite are not from historic eruptions and we do not know their exact localities. We also do not have measures of the crystallinities of these samples before totally melting them during the high pressure experiments reported in the manuscript.

Line 221. The precision of what method of vesicularity measurements? What sample is the 0.503 porosity measurement from?

Response: We have now specified that at this point in the manuscript we are discussing the precision of vesicularity measurements using X-ray microtomography. Baker et al. (2011), specifically investigated the reproducibility of porosity measurements using X-ray tomography, and the sample used in that study is unrelated to those studied in this manuscript. We have rewritten this sentence to make this more clear (lines 245-248).

Line 223. Why do you expect similar uncertainties? Vesicularity is dominated by large bubbles. Pore throat numbers and sizes are not necessarily dictated by largest bubbles.

Response: Similar uncertainties are expected because the same techniques are used for the measurements and in many cases the sizes are similar. We have rewritten this sentence to make this more clear (lines 250-251).

Line 239. How are branch diameters calculated/estimated? If I understand correctly, branch diameter $=$ pore throat diameter. In $1 \mathrm{~h}$, each branch looks like a line with single pixel width. Are pore throats define by maximum inscribed cylinders that connect spheres?

Response: Figure $1 \mathrm{~h}$ only shows the skeleton, which by definition is 1 voxel in width. The pore throat diameters are defined by the diameter of a sphere that can be fit through their narrowest part. We do not define the length of the pore throat so the idea of fitting them with a cylinder is not valid We have explained our techniques more fully on lines 254-259 of the revised manuscript. Please also see the caption to Figure 1.

Line 240. Can a cartoon be added here to help the reader follow this discussion? How does pore shape affect the overlapping sphere distinction? What if pores are nonspherical? Are all pores that have begun to coalesce then grouped as a single pore?

Response: We have not included a cartoon, but in the revised text refer the reader to Figure 1 in the revised text (lines 270, 274-275). The steps in this process are graphically shown in the example presented in Figures $1 \mathrm{~d}-1 \mathrm{~h}$.

Line 243. What is the formula for degree of inflation? What does 'the amount of inflation can be controlled' mean? Is the inflation degree a proxy for the size of bubble centered on each node? Is this inflation process isotropic?

Response: Spheres were inflated isotropically; this is now stated in the revised manuscript (line 271). The papers cited in this section of the manuscript provide many more details of the techniques used for the measurements used in this study than can be included in this manuscript.

Line 247. What does maximum inflation mean? Further, you state that "this parameter only weakly affects the computed values"... computed values of what?

Response: Maximum inflation is largest sphere that would fit the bubble, but this size sphere can underestimate the number of bubbles (as written on lines 245-246 on the previous version of the manuscript), so typically a value less than the maximum is used, as explained in the manuscript. The "computed values" are those of the bubble and throat numbers and sizes. This is now stated in the revised text on lines 257-258.

In Figure 1h, it looks like the use of spheres that completely fit within bubbles creates 
underfit bubble sizes. So bubble sizes are minima? But pore throat sizes may be maxima? (Line 252). Is that correct?

Response: No, bubble sizes fit to the maximum of the pore and so are the average pore throat sizes because the same techniques are used to measure both (see lines 227-230 in the previous version of the manuscript and lines 255-259 in the revised version). This is a standard technique. We have changed the sentence on lines 255259 to make this more clear. All details of the techniques are provided in the reference at the end of the sentence in the manuscript (Hildebrand and Rüegsegger 1997), which is a often-used reference for such measurement techniques ( 1435 citations as of 20 April 2019). Another of the standard references cited in this portion of the text is Lindquist 2002 (45 citations).

Line 277. This may be simpler to read if written in equation form.

Response: Great idea. We have made the change (line 309-313 of revised manuscript).

Line 296. In the Bai et al. study, it appears that the difference between modeled and measured permeability is minimized at $<65 \%$. Where does $50 \%$ come from?

Response: We only chose $50 \%$ for comparison because we thought the reader might be interested in the comparison of measured and modeled permeabilities in the exact center of the permeability range. Obviously, this comparison is confusing the reviewer and will probably confuse many readers so we have deleted the sentence.

Line 298. From Bai et al. "The ability of simulations to predict the macroscale Darcian permeability is limited by two independent characteristics. The first is the intrinsic grid resolution, as measured by the physical voxel size a with respect to the characteristic length of the pores (e.g., as determined by bubble size, surface curvature and surface roughness). The second is the physical sample size (quantified by the product of the number of lattice points along each edge NL and the voxel size a ), with respect to the geometrical correlation length (e.g., as determined by bubble connectivity and volume fractions)". This statement suggests that the important parameter is the ratio of voxel edge length to edge length of subvolume with respect to geometrical correlation length, essentially a measure of sample heterogeneity. How does that play in here?

Response: The intrinsic grid resolution of these measurements is at least as fine as that used in Bai et al. (2010) because the of the higher resolution imaging and, in most cases similar ranges of bubble sizes and sample sizes used for lattice Boltzmann simulations. Additionally, the mean bubble diameters in this study are similar, or smaller, as those measured in Bai et al. (2010), indicating that the geometrical correlation lengths in both studies are similar. Therefore, the arguments in this section of the manuscript support the use of lattice Boltzmann permeabilities based upon the findings of Bai et al. (2010). Please also note that, following the Editor's suggestion, this section of the manuscript has been significantly condensed in the revision.

Line 310. Even if there are no anomalously large bubbles, isn't the controlling factor the range in bubble size distribution between subvolumes?

Response: One of the controlling factors is the range in the bubble size distributions between different subvolumes, but the importance of pore throat size distributions is stressed in this manuscript. Please note that in order to save space this sentence has been deleted in the revised manuscript.

Line 315. In order to apply the test for effect of lattice size used in Bai et al. 2010, don't the geometrical correlation lengths need to be similar? Is that so?

Response: Yes, please see response above to comment on line 298.

Line 319. Does no significant difference with respect to orientation mean that permeability values were within a factor of 2 of each other? Factor of 3 ?

Response: We have explicitly state what we mean by a "significant" difference, a 
factor of 2 on line 358 of the revised manuscript.

Line 327. Does this mean that there was a delay? Was the delay due to heating time? Or was there a further delay after the sample reached $\mathrm{Tg}$ ?

Response: We could only estimate minimum glass transition temperatures at the heating rates used for these experiments. We have changed this discussion concerning nucleation delay in the revised manuscript (lines 438-442)

Line 329. What is 'onset of glass transition temperature', do you mean simply 'glass transition temperature' or onset of ductile behavior?

Response: The glass transition does not occur at a single temperature but over a range of temperatures that is influenced by the heating, or cooling, rate (Moynihan et al., Journal of Physical Chemistry, 1984, 78:2673-2677; Giordano et al. 2005-reference in the manuscript). The onset is the first evidence of the glass transition during heating as shown in Giordano et al. (2005--reference in manuscript). We have referenced Giordano et al. (2005) after use of this terminology in the manuscript (Line 428)

Line 362. Put in a distance here...? How many um?

Response: We have now put in the approximate distances away from the edges. See line 466 of the revised manuscript.

Line 365. This is true for each individual sample, not for aggregate of samples.

Response: We have now started this sentence with the words "In general, in each sample the ". Line 470 in the revised manuscript.

Line 400. Perhaps add a clause to the beginning of this statement, like "If the 2 samples can be thought to represent points on a single evolutionary trend". But note that bubble size doesn't even increase between basaltic runs (see graphs in my attached excel file).

Response: We have added the suggested wording (line 513-514 in the revised text). We agree that the bubble size does not increase between the two runs, in fact the maximum bubble size and the average bubble size decreases as shown in Figure $3 a$.

Line 418. "evenly distributed" meaning what?

Response: We have rewritten the sentence on this line to make the meaning more clear (lines 534-536 of the revised manuscript).

Line 422. Using a single bubble as indicative of process is less convincing than multiple... can you broaden the size bin to $<15$ um?

Response: We prefer to keep our bin size at approximately the same value as the imaging resolution. However, we agree that one bubble is not convincing (which is why we specifically stated that the interpretation was based upon one bubble in the previous version of the manuscript). We have slightly modified the sentence to indicate that the one bubble "suggesting continuing bubble nucleation" (line 539-541 of the revised text).

Line 425. Is this the spatial density?

Response: Yes it is because the figures are bubble number densities. To make our meaning of the sentence more clear we have added "in the same sized volume" to the sentence (line 543).

Line 505. What is the uncertainty on permeability measurements? Have you tried calculating permeability of 2 subvolumes in the same sample in order to characterize variability? Is the difference between these permeabilities actually 'significant'? 
Response: Our uncertainties in the permeability measurements are discussed on lines 348-352 of the revised manuscript. We have not calculated the permeabilities on 2 subvolumes from the same sample because our goal was to fully characterize the subvolumes we thought best represented each sample's center, not investigate the variations in the sample properties (see lines 205-206 in the previous version and lines 230-231 in the revised manuscript).

Line 507. Why surprisingly?

Response: We wrote "surprisingly" because the lowest porosity dacitic foams display lattice Boltzmann permeabilities similar to the andesites but the two highest porosity ones do not (see Figure 7). We have deleted the word "surprisingly" from the revised text (line 637).

Line 515. Incomplete sentence fragments. This paragraph from Line 510 on is discussion and should be removed from this section.

Response: This "paragraph from Line 510" presents our observation that viscosity does not appear to simply control permeability at equivalent porosities. We have moved this paragraph into the beginning of the Discussion section (Lines 669-676 in the revised version).

Line 519. This paragraph is the place for general observations that could be displayed in figures. For example, in single sample experiments as average bubble sizes increase, pore throat increases, in some cases linearly (see andesite sample for example). In general, pore throat diameters are about half the diameter of bubbles (using values from Table 2, see excel file). What are the implications of this relationship? Figure $1 \mathrm{~h}$ doesn't seem consistent with pore throats being half the diameter of bubbles though...

Response: We prefer to keep the simple summary paragraph at the end of the "Results" section on lines 519-527 of the previous manuscript. We don't want to discuss the average bubble sizes and pore-throat diameters because we don't think the averages are meaningful and only included them so that readers can compare those values with similar values published in other studies. Figure $1 \mathrm{~h}$ does not present all of the pore throats found throughout the sample, which is why the average porethroat diameters do not appear to be $1 / 2$ those of the bubbles.

Line 532. Mention the other controlling variables here that you discuss later.

Response: We have done this in the revised text (lines 663).

Line 536. Be clear here that you are discussing experimentally reheated aphyric basalts and dacites, not basaltic and dacitic eruptive products in general.

Response: This line has been deleted in the revised text.

Line 546. The previous discussion does not address the fit of new data to power-law relationship in general. The dataset presented here appears to follow a non-power law trend. In the attached excel file, an exponential fit to the andesite porosity-permeability appears to fit the data better. Why would that be? Is it due to experimental reheating in contrast to natural decompression? Note that other experimental reheating of clasts has led to bubble collapse in other experiments (Kennedy et al. 2016).

Response: We agree with the reviewer that the andesitic data can be better fit with an exponential relationship, but there is no theoretical foundation of which we know to support such a fit. On the other hand there are theoretical foundations for a power-law fit and that is why we chose such a fit. We are aware of the Kennedy et al. study on the collapse of porous samples, but think that discussion of it will not significantly enhance this manuscript and will unduly lengthen the manuscript.

Line 573. "appears to account for..." - can you quantify this relationship instead? Use a plot of BND? 
Response: No, we cannot quantify the relationship between the high bubble number and pore throat number densities of the trachyandesitic sample with a porosity of 0.35 in any meaningful way. However, we do present the BND and the PTD in Table 2 and display the size distributions in Figures $5 \mathrm{a}$ and $5 \mathrm{~b}$ of the manuscript. Note that this sentence has been deleted from the revised manuscript.

This section is verbose; shortening the text would add clarity.

Response: The section has been shortened.

Line 575-600. Repetitive of the section at Line 475; simplify and condense. Do you have data to support determination of percolation threshold?

Response: We have modified this section in response to this comment, but think that the discussion of percolation theory and its predictions of interconnectivity of objects is needed. The data for the determinations of the percolation threshold of random objects in 3 dimensions is provided in the references provided in this part of the text. Our studies have not identified a percolation threshold in the samples investigated; all of our experimental samples are permeable, with the exception of one sample containing a single bubble and another whose permeability could not be measured due to its extremely fine structure. However, we reference other studies of permeability in magmatic foams that have found percolation thresholds in both the previous and revised version of the manuscript.

Line 581. "statistical nature of percolation threshold" - what does this mean?

Response: Mathematically, true percolation thresholds are only exact for infinite systems. Finite systems can display variations about these thresholds (as discussed in the references on line 582 of the previous version of the manuscript). We have modified this sentence to make this more clear (Lines 747-751 in the revised text).

Line 606. I don't think you can generalize about basaltic bubble growth from these four distinct runs (as in attached excel sheet that shows no bubble size pattern between the 4 volumes). This is an over-interpretation of this increase.. delete Lines 606-612?

Response: We think that it is important to discuss these experimental results on the basaltic bubble growth and we conclude this paragraph by stating that we do not think the permeability increase between 0.5 and 0.55 porosity seen in the basalts is significant, so we are in agreement with the reviewer. We also note that while the mean bubble sizes in experiments at 0.5 and 0.55 are similar, the bubble size distribution changes significantly (Fig. 3a).

Line 622. Again, this could be combined with discussion above and shortened significantly. If your data does not add to the discussion - delete?

Response: We have made the combination as suggested by this reviewer (new version on lines 731-742).

line 692. Delete sentence "Such a calculation..." - unnecessary.

Response: We have removed the sentence and modified the previous on slightly (Lines 883-887)

Line 728. Add demonstration of the improvement of this model over the DeGruyter or Burgisser models for all of the samples plotted here (Fig. 9C).

Response: We have modified the text above line 728 in the previous version of the text to indicate that our quantitative comparisons are for all of the samples, except for when explicitly stated otherwise (Lines 920-923). We do not have the data for the Bai et al. (2010) samples to make a comparison with the Degruyter et al. and Burgisser et al. models. We have now added a sentence comparing the results of this model to the results reported by Burgisser et al.

Line 732 . Why 0.3 ? 
Response: We chose 0.3 because it is the sphere percolation threshold and have now specified the reason this in the revised manuscript. This paragraph has been moved to earlier in the section following the suggestion of the Editor (Line 872).

Line 738. You say that you concur; on what basis? Or perhaps rephrase.

Response: We have rephrased this sentence. Our reasons for concurrence are detailed later in the same paragraph (line 937-942 of the revised manuscript).

Line 749. Again, without an estimate of uncertainty of your permeability calculations, the relative difference between samples is difficult to characterize.

Response: The uncertainties in the permeability calculations were stated in the experimental techniques section (lines 315-317 in the previous version of the manuscript) and in this new version (lines 348-352). Additionally, the caption to Figure 7 states that the permeability uncertainties are "typically the same size, or smaller than, the symbols".

Line 757. What composition is this melt film thickness threshold estimated for? And is that for crystal-free melt?

Response: We have specified that the melt composition was rhyoltic in the revised text (line 958 in the revised text).

Line 771. Perhaps 'compared' is more apt than 'correlated' here... several authors examined covariations between the two parameters in order to better understand the role of path effects on permeability.

Response: This section on inertial permeability has been deleted. Nevertheless, we think that because the "earlier" studies produced mathematical relationships between viscous and inertial permeability the use of the verb correlated is correct.

Line 774 . Is it only pore shape and size? Could it not also be connectivity?

Response: This line (entire section) has been deleted from the revised manuscript

Line 777. What composition of pumice? Crystal-rich or crystal-poor?

Response: This line (entire section) has been deleted from the manuscript.

Line 783. As above - is that for all data? Composition? Crystal content?

Response: This line (entire section) has been deleted from the manuscript.

Line 793-7. It's not clear why these sentences are here. Inertial permeability is not considered in modeling later in paper. Perhaps remove these sentences and justify lack of inertial permeability in models.

Response: This line (entire section) has been deleted from the manuscript.

Line 847. Again, why use all data lumped together? You state earlier that there are many controls on permeability, including crystal content and bubble growth rate. Given these variations, why not simplify the discussion in this paper and focus solely on aphyric samples with bubble growth rates that you think are similar to your experiments?

Response: This line (entire section) has been deleted from the manuscript. In the revised manuscript we only used aphyric-to-crystal-poor samples.

Line 869. How do these results present an improvement over the Namiki and Manga (2008) conclusions? What is the take-home message from using different porositypermeability relationships than the Rust and Cashman formulation? 
Response: This line (entire section) has been deleted from the manuscript. In the previous version of the manuscript we used the model of Namiki and Manga to demonstrate to the reader that only high-permeability magmas would be expected to lose their driving gas and instead that most magmas would retain it. This was not done by Namiki and Manga. The difference from the Rush and Cashman formulation is that the equations provided in the manuscript demonstrate the wide range in permeability possible at a single given porosity.

Line 885. This introductory sentence to your conclusions is in contrast to your application of general porosity-permeability relationships that encompass all data from the literature, without separating datasets based on your assessment of the factors that control permeability most strongly.

Response: Our application of the general results was not to demonstrate that they could be applied to any specific magma, but instead to show that they constrained the range of possible permeabilities at any specified porosity. In the previous version of the manuscript we used the model of Namiki and Manga to demonstrate to the reader that only high-permeability magmas would be expected to lose their driving gas and instead that most magmas would retain it.

Line 891. This study does not prove a primary bubble growth rate control on permeability.

Response: We have changed this line to state that our results "are consistent with" (line 1093 in the revised manuscript).

Line 896. Is this a remarkable finding of the original Namiki and Manga paper or one asserted for the first time here?

Response: This is a remarkable finding of this work. But we have deleted this line because we no longer discuss the Namiki and Manga model in the revised manuscript.

Table 2. Note that average bubble diameter and average throat diameter for dacite samples are the exact same numbers - one of the columns must be incorrect for dacite samples?

Response: Yes, this was an error that has been corrected and for which I apologize.

Figure 1. Add a scale bar parallel to the front face of imaged volumes.

Response: Done

References:

Kennedy, Ben M., et al. "Surface tension driven processes densify and retain permeability in magma and lava." Earth and Planetary Science Letters 433 (2016): 116-124.

Reviewer L. Chevalier

Dear editor,

I have carefully read the paper from Baker et al., and detail in the following letter my comments and

suggestions on their work. I hope this will help you to decide whether it should be published in the Bulletin of Volcanology.

The work presented in this paper aims at better understanding the diversity of permeability-porosity relationships from the analysis of the foam structure for samples of basaltic to dacitic compositions. In-situ micro-tomography scans of vesiculating basaltic to dacitic foams were analyzed for characterizing the foam structure, and used for estimating the samples permeability from lattice- Boltzmann flow simulations. The resulting data provide the chance to follow the evolution of the foam structure and sample permeability with time for these different compositions, which would be of great interest to the readership of the Bulletin of Volcanology. The analysis of these data 
highlights the dominating role of large pore-throats in permeable flow. The study also supports the idea that the bubble growth rate impacts significantly the development of permeability. Readers interested in magma permeability development and degassing processes will be particularly interested in these results. Finally, the study provides results from a numerical model that demonstrate that only the most permeable magmas would be able to lose gas while ascending in the conduit. This is of high interest to readers interested in understanding the evolution of eruption intensity and its link with gas loss.

Since I got the chance to review a first version of this paper about a year ago, I could notice important changes and improvements in the paper:

- The methods section gained a lot in clarity and precision, and is now very easy to read. The results section and the first parts of the discussion were also intensively revised.

- Although the importance of pore throats for understanding the porosity-permeability relationship remains a result of first importance, it is now suggested that the maximum pore- throat diameter, rather than the cumulative pore-throat area, should be used for estimating permeability.

-The influence of the melt composition on permeability development became of minor importance in this new version, and bubble connectivity is much less highlighted.

In general, the research is presented in a clear and accurate way, with developments of high interest and potentially high outreach. I feel, however, that the soundness and coherence of the article could be improved to emphasize and strengthen the ideas developed here, and increase its impact in the following way:

1.Considering the important changes in the paper, I think that the introduction should focus more on the link between foam structure and permeability, and less on the question of composition, which looks to be no more priory in the rest of the article.

Response: We have made the suggested changes in the Introduction (lines 98-106 in the revised manuscript).

2. The experimental procedure, which provides the exiting possibility of following permeability evolution with time, would worth some more discussion to highlight its complementarity and differences from the more common decompression experiments.

Response: We have added an entire new section comparing our isobaric heating experiments to the more usual isothermal decompression experiments (lines 364-418)

3.Using the maximum pore-throat diameter as the characteristic diameter for permeability raises some concerns to me that I detail below. In my opinion, some questions need to be answered and discussed to strengthen this interpretation of the data and its future impact in the community.

These main comments are detailed below, and other comments and suggestions are listed in the following pages. Overall, I think that this paper, providing some modifications, has the potential to be a great contribution to the understanding of magma permeability evolution. I therefore recommend considering it for publication after a moderate revision. Laure Chevalier

Main comments

Introduction

Considering the changes made in this new version of the paper, I think that the introduction needs some adaptation to refocus on the message delivered in this paper and emphasize its importance. Although the introduction addresses the very interesting question of the influence of melt composition, it is no more a priory result in this new article version (see the other comments section below). Conversely, the influence of the pore-throat maximum diameter, which is a key result, is emphasized in the introduction. I think that focus should be redirected to introduce and emphasize the message delivered in the article. Besides, a review of the influence of porous network 
parameters in permeability development would strengthen the introduction of the work on foam structure presented here (most of the relevant references are already cited in the article).

Heating procedure for bubble growth

With increasing heat, melt viscosity decreases. Lindoo et al. (2016) conclude from experiments using various melt compositions that viscosity has no effect on the percolation threshold, but may significantly impact permeability. Melt viscosity evolution with heating raises some questions on the experimental procedure that would worth some discussion:

- Could you estimate the influence of this viscosity evolution on bubble coalescence and permeability development along with porosity increase ? Does the bubble growth rate increases as the temperature increases? Could you estimate the relative importance of such viscosity changes (after vesiculation starts), compared with differences of viscosity due to composition?

Response: We have added an entire new section comparing our isobaric heating experiments to the more usual isothermal decompression experiments (lines 364-418) We see no evidence of increasing bubble growth rate as temperature increases. We add some estimations of viscosity changes during heating and vesiculation, but state that there are uncertainties in the values for intermediate temperatures and time (lines 373-378).

- How far is viscosity limited bubble growth (vesiculation due to heating) comparable with solubility controlled bubble growth (vesiculation due to decompression) ? What about pore throat development in each condition?

Response: We have added an entire new section comparing our isobaric heating experiments to the more usual isothermal decompression experiments (lines 364-418)

- If temperature was maintained constant at the end of the rhyolite experiment, would bubbles have been visible after some time?

Response: No. The sample could not be heated because it did not absorb the laser radiation. This is written in line $213-215$ of the revised manuscript:

\section{Maximum pore throat diameter}

Using the maximum pore-throat diameter as the characteristic diameter for permeability raises some questions to me.

- How well is this maximum pore-throat diameter representative for the sample ? If it is the real maximum value (of a single pore), increasing the sample size may favor the presence of an even larger pore throat, leading to an increased sample permeability ? What if the larger pore throat is in a series with very small ones?

Response: The maximum pore throat diameter in the specific subvolume analyzed is characteristic of that subvolume, and that subvolume was chosen to be representative of the central portion of the sample (far from sample surfaces). We agree that increasing the largest pore throat would increase the sample permeability at constant porosity as our model indicates. Such a problem also occurs using the model of Degruyter et al. (2010) or of Burgisser et al. (2017), where if a larger volume was to be chosen and a larger average pore throat diameter is found the permeability would increase. If the largest pore throat is in series with very small ones, then the small ones would control the permeability, however our analysis of the samples indicates that there are mulitple pore throat connections between bubbles and that permeability correlates with the largest pore throat. Regretfully, our samples are too small to chose significantly larger volumes.

- Playing Devil's advocate: Would andesitic permeability retrieval also fit that well if the volume location had changed between the different measurements? Is there any risk that the importance of the maximum pore throat is a singularity present for this experiment that would reveal not so good for others? 
Response: It might, but we could not effectively test this as we chose the largest volume possible that we thought was representative of the sample's center for all of the measurements and the lattice-Boltzmann simulations of the permeability.

- If, instead of the maximum throat diameter, a value deduced from bubble size distribution (e.g. 90 th percentile) was used, would results still be satisfying ? How does the number of large pore throats affect permeability?

Response: We did not test these hypotheses in this manuscript because they are beyond the scope of this manuscript. We would not be surprised if the 90th percentile distribution of the bubble sizes would produce a satisfying model and expect that if a sample has many large pore throats that the permeability at equal porosity would be greater than for a sample with only one large port throat in parallel with smaller ones.

- I compared the maximum pore-throat diameter value obtained from bubble average diameter using eq. 6 with actual pore-throat average sizes, and 84th percentile value for pore-throat sizes (meaning that $84 \%$ of pore sizes are below this value, and the rest is above), given for isotropic samples in Burgisser et al. (2017, Supplementary Information). The values obtained with eq. 6 range from -8 to about $30 \mu \mathrm{m}$, and correlate rather with the average pore-throat size than with the 84th percentile size given in Burgisser et al. (2017). Have you tried estimating permeability for the Burgisser et al. (2017) samples using the 84th percentile value for pore-throat size value they give? How would fig.9c be modified?

Response: In think the reviewer is referring to the 84th percentile of the bubble diameter, not the pore diameter. The Supplementary Information for Burgisser et al. (2017) does not include the 84th percentile for pore throat sizes, instead they only show the 84th percentile for bubble (pore) sizes. Additionally, we found a typo in Equation 6 of the older version of the manuscript (now corrected and now Eq. 9 in the revised version). This mistake of ours led to the low values calculated by the reviewer. The included table is the comparison of the average bubble size (which we used-see line 714 in the previous version of the manuscript), the mean bubble size, the average bubble size of the 84th percentile, and the throat diameter from Burgisser et al. (2017) together with the estimated maximum pore throat diameter from Equation 9 of the revised manuscript.

This table supports the reviewer's conclusion that the maximum pore throat calculated by our equation is similar to the average bubble diameter in the 84th percentile. Thus we expect that using the diameter of the $84 \%$ percentile would yield results similar to using the calculated maximum throat size. However, we have not done this because the $84 \%$ percentile is rarely reported in the literature and to our knowledge is rarely measured, whereas we chose to use the average bubble size to estimate the maximum throat size because the average bubble size is commonly reported.

Other comments

quotation comment

Lines 51-52: Connectivity [...] for each sample correlated positively with porosity and permeability. Although this result is really interesting, it is only implicit in the rest of the article (Lines 462-465 "The relationship between increasing porosity and decreasing tortuosity ... and a correlation between increasing connectivity and decreasing tortuosity" and Lines 525-527 "Increasing vesicularity increases connectivity and decreases tortuosity. All of these changes in foam structure result in higher permeabilities that are not simply related to the melt compositions investigated."). Could you highlight this result in the article by giving more details on your observations of connectivity-permeability correlation?

Response: We have now included Supplementary Figure 1 that presents the connectivity-porosity relations for each sample and therefore, because permeability increases with porosity (Fig. 7) an idea of the connectivity-permeability relations. Supplementary Figure 1 demonstrates that increasing connectivity is, in general, associated with increasing porosity (and permeability) for each of the compositions studied. However the relationship is not clear because high permeability basaltic samples have lower connectivities than lower-permeability andesitic ones (Supplementary Figure 1 and Figure 7). Because of this lack of simple correlation we 
chose not to expand our discussion of connectivity-permeability relationships in the text to minimize the length of the manuscript.

Line 75 : The competition between magma expansion due to exsolution of gas, and the loss of that gas ...

In natural systems, magma expansion is also tightly linked to gas expansion due to decompression.suggest to rephrase as : « magma expansion due to gas exsolution and inflation »

Response: We have made a change similar to the suggested one (line 82 in the revised manuscript).

First paragraph :

The first paragraph of the introduction addresses the very interesting question of the influence of melt composition on bubble network and permeability development. Having four different compositions is a strength of this article, however in this new version the question of its influence on permeability development is not priory, and results do not evidence a strong influence on permeability. The evolution of connectivity and bubble connection with composition is no more addressed, and as the maximum pore-throat diameter is now used instead of the cumulative pore throat area, its implication for permeability development looks very minor. I would therefore suggest either to refocus the introduction on the more general influence of porous network parameters, or to give more visibility to the observed influence or absence of influence of composition in the rest of the article (for this last option, it would be useful to add the connectivity and connection figures in supplementary material, for example).

Response: The Introduction has been revised in response to this suggestion. We have now added a supplementary figure with the connectivity versus the porosity to the manuscript.

Lines 98-100 : This study arose from the hypothesis that foam properties, including the bubble size distribution, tortuosity, the number and size of pore throats, and the degree of interconnectivity between bubbles, play an important role in controlling the permeability of magmatic foams.

The introduction should put into perspective and emphasize the importance of the work presented. A lot of work has been done for studying this link between foam structure and permeability development, and should be reviewed here. Most of the relevant references are already cited in the introduction. However, the enhanced understanding of the link between permeability and foam structure through these studies should be precised here. The reader needs to understand what has already been done, what questions arose from it, and what still needs to be understood,that the particularities and interesting results (about pores-throats) of the study presented here, as well as the way it contributes to precedent work are emphasized.

Some references supporting the link between composition and foam structure would also welcome here.

Response: We have tried to succinctly inform the reader of previous research demonstrating the importance of parameters other than just porosity that are important in controlling the permeability (lines 98-106).

Lines 107-109: All attempts to study a rhyolitic melt failed due to the inability of the laser furnace used in the experiments (Fife et al. 2012) to heat this composition to temperatures high enough for gas exsolution and bubble formation.

What would be a temperature high enough for rhyolite melting and bubble expansion ? Would a rhyolite with more water have been able to vesiculate at lower temperatures?

Response: The rhyolitic sample would simply not attain temperatures necessary for bubble growth. This has now been stated on line 215 of the revised manuscript.

Lines 110-111: The viscous permeabilities of the foams were determined from tomographic reconstructions of sample volumes using lattice-Boltzmann techniques Non-specialist readers may think that lattice-Boltzmann techniques were used to 
reconstruct sample volumes from tomography. Adding a coma after "sample volumes" may be enough for dissipating any doubt.

Response: This sentence has been revised to make our techniques more clear to nonspecialists (line 128-129 in the revised manuscript).

Lines 121-122: We further demonstrate that characterizing the structure of the foam, and particularly the size distribution of pore-throat diameters and their cross-sectional areas, is critical

I think this result concerning pore throats should be more emphasized in the introduction. (cross sectional area, however, is no more exploited in the paper).

Response: We have modified the introduction to stress the importance of pore throats (Lines 99-106 of the revised manuscript).

Lines 137-138 : trachyandesite and dacite contained $3 \mathrm{wt} \%$. water. The only successful experiment with the andesite contained $5 \mathrm{wt} \%$ water.

Could you precise how the water concentration is calibrated?

Response: We have now stated that the water concentrations are based upon the weight fractions of water used in the synthesis experiments (lines 159-160 of the revised text).

Lines 177 - 178 : Data acquisition was initiated at the first visible onset of vesiculation, bubble formation, and sample expansion.

Do you mean that you look for the first onset either of vesiculation or bubble formation or sample expansion, or that bubble formation and sample expasion are visible onsets of vesiculation ? In the first case, I suggest to replace "and" by "or" In the second case, I suggest to replace the coma after vesiculation by ":", or add "such as" before bubble formation.

Response: We have followed the reviewer's suggestion (line 201 of the revised manuscript).

What is the difference between vesiculation and bubble formation?

Response: None in this case. Following the reviewer's advice from the previous comment we think we have made this clear in the revised manuscipt.

Line 197 : These experiments span much of the range of porosities Are these different porosities obtained because of different ending conditions (then it should be mentioned) or because there was, for example, some gas loss ?

Response: Bubble growth stopped due to gas loss from the sample. This has been added in the revised manuscript (line 222).

Line 232-233 : Objects, bubbles and pore throats, were not counted unless their size was greater than two voxels.

Could you recall here the size of a voxel $\left(2.89 \times 2.89 \times 2.89 \mu \mathrm{m}^{\wedge} 3\right)$, to facilitate fig. 3-6 reading?

Response: We have followed the reviewer's suggestion (line 245 of revised manuscript).

Line 236 : The geometrical determination of pores and throats is ...

The word "pore" is sometimes associated with bubbles, and sometimes with porethroats, which is a little bit confusing, especially in this paragraph and in the following. Would it be possible to homogenise this in the article, so that "pore" is always associated either with "bubble" or with "pore-throat" ? If the presence of this word here is necessary for explaining the measurement method, it may be helpful to replace it by another synonym that does not appear elsewhere in the article (e.g. vesicle, hole, void, space).

Response: We have gone through the manuscript to ensure that the term "pores" is 
Lines 252-254 : Practically, this means that the throat-size distribution may present some large- valued outliers due to the consideration of branches that do not represent physical channels.

Could you give more details on how throat sizes are estimated ? Are these large-value outliers removed before taking the maxium pore throat diameter value for permeability calculation

Response: We have now provided more details on how the throat sizes are estimated (lines 255-259 in the revised text). There is one basaltic experiment with 0.55 porosity that contains one pore throat that is anomalously larger then the next largest one (see Fig. 3b). Whereas we cannot completely discard the possibility that the largest pore throat in this sample is an artefact, we think that size difference between the largest pore throat and the others in the distribution is not sufficiently large to discard the measurement (lines 504-509 in the revised manuscript). Please note that the latticeBoltzmann permeability determinations do not involve the measurements of the pore throat diameters and are performed directly using the tomographic reconstructions. Also note that if we use the next-largest pore throat for this experiment in our modified Burgisser model, the estimated viscous permeability is still within 1 log unit of the Lattice-Boltzmann permeability.

Lines 255-256 : The final assessment of pore (bubble) size is based on the diameter of the maximal inscribed sphere centered on the center of mass of the cluster of the overlapping inflated bubbles (Fig. 1H ).

and Lines 1109-1111: The size of the bubble to be counted is determined by a maximal inscribed sphere that is centered on the center of mass determined from the merged bubbles.

Might this method underestimate the bubble size ? If yes, what is the error made using this method? If you sum up the bubble volumes obtained this way, and then divide it by the total sample volume, do you get back to the measured porosity?

Response: No, the method of maximal inscribed spheres is the standard method used when a skeletonization approach is used to assess the bubble size distribution (see Hildebrand and Rüegsegger 1997 for details of the method-reference in text). The sum of the bubble volumes divided by the sample volume do not yield the total vesicularity because our techniques do not measure the volume of the pore throats only their diameter.

Lines 326-327 : No evidence of a significant nucleation delay (i.e., longer than a few seconds) was detected in any sample.

If there was any, what would you expect to see ? What are the criteria that you use to estimate whether there is a delay or not?

Response: We have deleted this sentence and discussed possible nucleation delay after the presentation of the estimated glass transition temperatures (lines 438-442 of the revised manuscript).

Lines 335-340: We estimate a minimum onset of the glass transition in our samples with $3 \mathrm{wt} \%$ water (MORB, trachyandesite, and dacite) at $460{ }^{\circ} \mathrm{C}$ and in the andesite with 5 wt $\%$ water at $440{ }^{\circ} \mathrm{C}$. However our heating rates were approximately 3 to 15 times more rapid than used in Giordano et al.'s experiments, thus the observed vesiculation temperatures were significantly above these minimum glass transition temperatures, in the range of 616 to $900{ }^{\circ} \mathrm{C}$ (Table 2).

Are these differences between the observed temperatures and the theoretical one due to observation limitations (e.g. determine vesiculation onset), in which case this should be mentioned, or should it be considered as a delay in vesiculation ? In this later case, this sounds contradictory with lines 326-327. Do you expect vesiculation as soon as the glass transition is crossed ? I think some clarifications are needed here.

Response: We clarified and moved our presentation of the glass transition and possible nucleation delays to lines $426-442$ in the revised text. 
Line 387 : the BNDs are in the thousands

BNDs are most often indicated in the literature as a number of bubbles per $\mathrm{m}^{\wedge} 3$. Here I understand that you talk about a number of bubbles per $\mathrm{mm}^{\wedge} 3$ (maybe because this is sample volume order of magnitude ?). This unit should be indicated here.

Response: The units have now been specified (line 492 in the revised manuscript)

Lines 387-388: The two samples with the lowest porosities (0.52 and 0.50 ) are from the same experiment and demonstrate that with time the number The fact that the samples with porosities of 0.52 and 0.50 are consecutive in time should be explicitely mentioned somewhere. For even more clarity, I think that the fact they are from the same experiment should be recalled in fig. 3 legend, otherwise it is not straightforward to understand why these samples are not sorted by increasing porosity.

Response: This has now been stated on lines $496-498$ of the revised manuscript.

Lines 403-404 : the sample volume quantified varied slightly between different time steps to avoid counting anomalously large bubbles.

What would happen if you considered such a sample (with anomalously large bubbles) and use the permeability formula with the largest pore throat size ?

Response: Although we haven't done this because we concentrated on using the most representative portions of the interiors of the samples, we think our model would work on any sample where the appropriate values for Equation 9 are known.

Line 406 : about andesite samples BSDs

For the three first andesitic samples presented in fig.4, the porosity evolves from 0.17 in the first sample to 0.28 in the third one. However, looking at the bubbles size distributions, all per $\mathrm{mm}^{\wedge} 3$, the number of bubbles observed in the first sample (porosity 0.17 ) is higher than that observed in the two other samples (porosities of 0.28 ), for every bubble size bin. Were the porosity and BSDs measured for the same volumes ? Were bubbles counted in the whole volume whereas porosity corresponds to connected porosity only ? This evolution is quite surprising, and I think some explanations would be welcome here (or in the fig.4 legend).

Response: There was a mistake in constructing this plot and it has now been fixed and the other plots checked.

Line 419 : a single bubble and Line 422 : one appears at $\Phi=0.64$

Would it be possible to mention the volume of the sample here (about $0.05 \mathrm{~mm}^{\wedge} 3$ ), so that it is more straitforward to make the link between this single bubble and the $\sim 20$ bubbles shown in fig.5?

Response: This has now been done on line 537 of the revised manuscript.

Lines 429-430 : up to a maximum of almost $400 \mu \mathrm{m}$ at $\Phi=0.84$ (Fig. 6a). and lines 436-437 : The PTDs of the dacitic sample demonstrate growth of larger pore throats up to a porosity of 0.87 followed by a decrease in that value as the sample reached $\Phi=0.84$ (Fig 6b).

It could be usefull to indicate in the fig.4-6 legends that the different panels correspond to successive times

Response: This has been done for the revised manuscript. Please see the caption for Figure 3.

Lines $445-446$ : values in the hundreds to thousands

The unit should be precised here.

Response: This has been done in the revised manuscript (lines 565-566)

Lines 449-456: The average coordination number (or number of bubbles surrounding a specified bubble) [...] and the maximum coordination numbers are often near 100 and can reach almost 600 (Table 2). 
Could you be more precise in the definition of the coordination number ? Does it count bubbles that are in direct contact with the specified bubble (and maybe connected)? Or does it count bubbles in the proximity of the bubble of interest ? In this case, up to which distance is a bubble considered to be in proximity?

Response: This was been specified on line 449 of the previous version of the manuscript as the number of bubbles surrounding a specified bubble. In the revised manuscript we have expanded our explanation of the average coordination number on lines 569-570.

Lines 462-465 : about connectivity

I remember that there was a figure showing connectivity results in the first version of this paper. Could this figure be added to the supplementary material ? I think this would be of interest for readers.

Response: We added this figure to the manuscript as supplementary material; we note that interested readers can easily construct it from Table 2 in the manuscript.

Lines $480-482$ : Permeability thresholds in natural and experimental magmatic foams can vary from below $\sim 0.03$ (e.g., Saar and Manga 1999; Bai et al. 2010) to values in excess of 0.63 (Lindoo et al. 2016).

The experimental data from Takeuchi et al. (2009) could also be of interest here : They studied magma permeability development from experimentally decompressed rhyolitic melts, and found that samples with a porosity below $80 \%$ were almost impermeable.

These data were fitted by Rust and Cashman (2011) (see their fig.5), with a power law relationship similar to the one you give (lines $475-476$ ), in which $\Phi$ is $78 \%$.

Response: The crystal-free and crystal-poor permeability measurements of Takeuchi et al $(2008,2009)$ have now been included. See also line 606 in revised manuscript.

Lines 575-600 : Percolation theory and particularly Lines 591-592 : Thus, the size distribution of spherical bubbles is expected to have a minor effect on the percolation threshold.

Although the percolation theory is a strong tool for understanding percolation and permeability development, it does not account for magma and bubble dynamics, nor for bubble interactions. I think that the limits of this approach should be discussed later in the section. How did natural and experimental observations temper this interpretation ? Particularly, Burgisser et al. (2017), found that the bubble size distribution does have a significant effect on percolation development in experimentally decompressed rhyolitic melts, which is contradictory with the results from Consiglio et al. (2003).

Response: A complete discussion of percolation theory and bubbles is far beyond the scope of this manuscript. Our goal was only to present the very basic aspects of percolation theory and compare its percolation thresholds to the experimentally measured ones. We have now added Burgisser et al.'s (2017) observations to our discussion of the effects of size distribution on the percolation threshold (line 764-766).

Lines 623-624 : Each specific sample may have its own critical porosity based upon the size distribution and shape of the bubbles and pore throats in the sample, as discussed above. And Line 639 : such as the size distributions of bubbles and pore throats

It looks like the conclusion above was that bubble size distribution should have a minor influence on the percolation threshold, which looks contradictory to the idea you express here. Or do you refer to somewhere else in the text? Could you indicate more precisely the section where to look for this discussion?

Response: Lines 575-600 are discussing the conclusions from percolation theory and lines $623-624$ and 639 are discussing what is seen the permeability measurements and what we think is affecting the permeabilities. We try to make clear in this section and in subsequent sections of the manuscript that percolation theory provides important ideas about the development of permeability with porosity, but that there are many details concerning bubble interactions that remain to be discovered. 
Lines 683-686 : Applying this paradigm, the porous network in foams can be envisioned as resistors interconnected to one another to create a continuous circuit that when the permeability threshold is exceeded allows fluid to flow across the sample. The connections between resistors in the network can be either in parallel or in series (Stauffer and Aharony 1994).

Blower et al. (2001) should also be cited here.

Response: Blower et al. (2001) has now been cited in the revised text on lines 877 , 881.

Lines 699-701: Based upon this observation, an empirical value of $X=10$ (5 times the original value of $X=2$ ) was chosen, and the resulting fit of the Burgisser et al. model to the data is remarkable (Fig. 9b), with all but one of the measurements reproduced to within one log unit and a chi-squared value of 0.01 .

$X$ refers to the permeable pathway circularity. In the case of spheres its value should be 2. Changing its value to 10 to fit the data looks confusing to me, why not introducing a fitting parameter instead ?

Response: In the revised text we have indicated that the value of 10 can be thought of as a fitting factor of 5 times the value for a circular cross-section on line 896 .

Lines 708-710 : Additionally, we found that the maximum throat diameter can be related to the average bubble size, davg bubble, by

The values obtained with eq. 6 range from -8 to about $30 \mu \mathrm{m}$ for Burgisser et al. (2017) isotropic samples, and correlate rather with the average pore-throat size than with the 84th percentile size given in Burgisser et al. (2017). See main comments.

Response: Please see our response in the main comments section of this reviewer.

Line 770 : A brief discussion of inertial permeability

Have you heard about this paper from Zhou et al. (2019) that came out very recently? It relates inertial and viscous permeabilities for a wide range of geological materials. It is much more general and less precise than the relationships you cite here, but could be of interest for introducing the general apparent existence of a link between viscous and intertial permeabilities.

Response: We have now deleted this section from the revised manuscript and discuss Zhou et al. (2019) in the introduction of the revised manuscript (lines 135-139). We have kept the discussion of Zhou et al. succint in our efforts to shorten the length of the manuscript.

Lines $872-875$ : Gas lost to the conduit walls would imply a larger volume gas loss that would still affect the permeability-porosity relations of the magmatic foam. Such gas loss through the conduit walls would have implications on transitions in eruptive style. Thus, the fundamental results of the Namiki and Manga (2008) model appear sound. These few sentences sound a little bit contradictory to me, as I understand that you frist say that gas loss to the country rock could have a significant influence, and then deduce from this that the model from Namiki and Manga (2008), that does not account for it, is sound. Could you explain why the model from Namiki and Manga (2008) is sound despite of not considering gas loss to the rock? Or rephrase or rearrange this parragraph so that in does not sound that contradictory?

Response: We have now dropped the section on the Namiki and Manga model.

Lines 1097-1098 : with a smaller 160160 pixel region used for quantitative measurements.

Is the $160 \times 160$ pixel region the one used for permeability measurements using latticeBoltzmann techniques ? It appears to contain very large bubbles (that cover in length more than half of the sample). Are permeability measurements still reliable in this case ? Blower (2001) provide evidence that the permeability-porosity relationship is independent on bubble and sample size as long as the bubble radius is smaller than $0.1 \mathrm{X}$ (with $\mathrm{X}$ the size of the volume considered), in the case of a mono-disperse size distribution. This can be interpreted as the sample dimensions should represent at least 5-10 bubbles to be representative. How many bubbles are contained in every 
dimensions of your samples ? Were any test on permeability vs size of the sample done? This may provide another argument for representativeness. Are such big bubbles present in every analysed sample, or only in the most porous ones? In this case, I would suggest, if available, to put images less controversial here (same sample at a lower porosity for example).

Response: The techniques used by Blower (2001) are significantly different from those used in this study and the constraints provided by Blower don't necessarily apply to the lattice Boltzmann simulations used in this study, although they are good starting guidelines. Instead, we have compared our simulations in terms of sample size, bubble fraction, and resolution to those of Bai et al. (2010) and found that we were within the range of those parameters where the results of lattice Boltzmann simulations are reliable. (see lines 334-355)

Lines 1099-1101: only considering larger interconnections between bubbles. e) The same reconstruction also considering smaller interconnections and main text. Could you give some more details on what is the difference between large and small interconnections ? What does the tunning parameter select ? Is it considering only connections when the pore throat is above a critical size ? What is the criteria that is changed?

Response: Practically the algorithm considers a downscaled (averaging multiple voxels together and replacing them by one pixel equivalent in volume to the sum of the averaged voxels) or upscaled version (finest resolution) of the volume. In a downscaled version of the volume, small interconnections disappear (and the algorithm is also faster). In an upscaled version of the volume (similar to using a digital zoom of a camera), small interconnection results are represented with more voxels and therefore the medial axis (which is a digital object having exactly one voxel thickness) can "pass through" that small interconnection. The changed criterion is the up- or down-scaling of the input volume. This has been explained a little more in the caption to Figure 1 in the revised text.

Line 1103 : pore throats (yellow) measured

What do you mean by pore throats here ? What is the pore throat size measured ? Is it the length of these yellow lines ? Or is it the diameter of the pore ? In this last case, how is it measured?

Response: The caption to Figure 1 has been modified to indicate that the yellow lines are the 1 -voxel skeleton. We do not measure the length of the yellow lines, nor does it make sense to do so because many of them are inside the bubbles, as shown by comparison of figures $1 \mathrm{~d}, \mathrm{e}, \mathrm{f}, \mathrm{g}$ and $\mathrm{h}$. Details concerning the measurement of bubble and pore throat diameters are provided in the text (see lines 254-264 in the revised text).

Lines 1104-1105: To reduce the number of over-counted nodes, spheres are centered at each node and inflated by differing degrees

Does this inflation process accounts for existing bubble walls? Or may bubbles close to each other, but not connected, merge artificially ?

Response: The inflation process accounts for bubble walls; bubbles close to each other, but not connected, cannot merge artificially.

Caption of fig.7 : The blue line (andesitic data fit) has no legend. Could you add some?

Response: The significance of the blue line has been added to the figure caption in the revised version of the manuscript (lines Figure 7 caption).

Figure $2 \mathrm{a}$ : Could you add a scaling bar of $1 \mathrm{~mm}$ on the first picture ?

Response: That has been done. 
Figure $2 \mathrm{~b}$ : A scaling bar would also be great here (the scale is not the same as in $a$, is it ?).

Response: That has been done.

Figure 3 : Could you precise in the legend that the first two panels are successive in time, and from the same sample?

Response: This has been done (revised manuscript Figure 3 caption).

Table 2 : Foot notes references on the table look to start at five (and I did not found the reference to footnote 1). Would it be possible to name them in a more instinctive way? References on table 1 are very easy to follow.

Response: This was my mistake in the previous version and has been corrected.

Typos add remove

Line 136 : capsules-CORRECTED

Line 301 : The ratio had no effect of on the permeability-CORRECTED

Line 688 : the Carmean-Kozeny-CORRECTED

Line 755 : The formation of a pore-CORRECTED

Line 768 : Lindoo et al. (2017) (about the influence of crystals)-CORRECTED

Line 791 : vary by only by about-CORRECTED/DELETED

Line 824 : Liu et al. (2005)-CORRECTED/Deleted

Line 1160 : either the Carman-Kozeny equation or the Carman-Kozeny equation of Degruyter et al. (2010) and that of Burgisser et al. (2017).-This sentence has been modified.

\section{References}

Blower JD (2001) Factors controlling permeability-porosity relationships in magmas. Bull Volcanol 63:497-504

Burgisser A, Chevalier L, Gardner JE, Castro JM (2017) The percolation threshold and permeability evolution of ascending magmas. Earth Planet Sci Lett 470:37-47

Lindoo, A., Larsen, J.F., Cashman, K.V., Dunn, A.L., Neill, O.K., 2016. An experimental study of permeability development as a function of crystal-free melt viscosity. Earth Planet. Sci. Lett.435, 45-54. http://dx.doi.org/10.1016/j.epsl.2015.11.035.

Rust, A.C., Cashman, K.V., 2011. Permeability controls on expansion and size distributions of pyroclasts. J. Geophys. Res.116.

Takeuchi, S., Tomiya, A., Shinohara, H., 2009. Degassing conditions for permeable silicic magmas: implications from decompression experiments with constant rates. Earth Planet. Sci. Lett.283, 101-110.

Zhou, J.-Q., Chen, Y.-F., Wang, L., \& Cardenas, M. B. (2019). Universal relationship between viscous and inertial permeability of geologic porous media. Geophysical Research Letters, 46. https://doi.org/10.1029/2018GL081413

\section{Author Comments:}

Note that two copies of Table 1 and of Table 2 have been included in this submission. One copy of each table is in .pdf format so that it can be easily read and the other copy is in .xls format 
Earth and Planetary Sciences McGill University 3450 University Street

Montreal, QC, Canada H3A 2A7
Sciences de la terre et des planètes Université McGill 3450 rue University Montréal (Québec) Canada H3A 0E8
Tel./Tél.: (514) 398-7485

Fax/Télé : (514) 398-4680

don.baker@mcgill.ca

http:// www.mcgill.ca/eps

3 May 2019

Professor Katharine Cashman

Editor of The Bulletin of Volcanology

University of Bristol

Dear Kathy,

Enclosed you will find our revisions to manuscript BUVO-18-00171. In response to the comments of the reviewers we have made substantial modifications to the manuscript and have reduced the Discussion from 19 pages to $\sim 11$.

We thank you for your continued evaluation of this manuscript for publication the The Bulletin of Volcanology.

Sincerely,

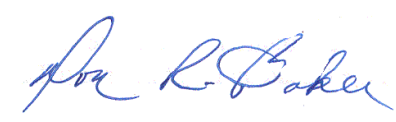

Don Baker, McGill University 


\section{The importance of pore throats in controlling the permeability of magmatic foams}

$14{ }^{4}$ Previously with: Laboratory for Synchrotron Radiation, Swiss Light Source, Paul Scherrer Institut, 155232 Villigen, Switzerland

$16{ }^{5}$ Currently: Department of Earth Sciences, University of Toronto, 22 Russell St., Toronto, M5S 3B1

$17{ }^{6}$ Department of Chemical Engineering, McGill University, 3610 rue University, Montreal, QCutebec, 18 H3A 0E5, Canada.

$19{ }^{7}$ School of Earth and Environmental Sciences, University of Manchester, Manchester, U.K.
Corresponding author: don.baker@mcgill.ca

1-514-398-7485

\author{
BUVO-S-18-00171 \\ Revised 3 May 2019 \\ 9 December 2017 \\ Revised 17 July 2018 \\ Revised 4 December 2018 \\ Revised April 2019
}




\section{Abstract}

46 Foam formation during vesiculation of hydrous magmatic melts at 1 atm was studied in situ by

47 synchrotron X-ray tomographic microscopy at the TOMCAT beamline of the Swiss Light Source

48 (Villigen, Switzerland). Four different compositions were studied; basaltic, andesitic, trachyandesitic

49 and dacitic hydrous glasses were synthesized at high pressures as starting materials and then laser

50 heated on the beamline. The porosity, bubble number density, size distributions of bubbles and pore

51 throats, as well as the tortuosity and connectivity of bubbles in the foams, were measured in three

52 dimensions based on tomographic reconstructions of sample volumes. The reconstructed volumes

53 were also used in lattice-Boltzmann simulations to determine viscous permeabilities of the

54 samples.foams. Connectivity of bubbles by pore throats varied from $\sim 100$ to $10^{5} \mathrm{~mm}^{-3}$, and for each

55 sample correlated positively with porosity and permeability. Although permeability increased with

56 porosity, the relationship is complex; consideration of the results of this and previous studies of the

57 viscous permeabilities of aphyric and crystal-poor magmatic foamssamples demonstrated that at similar

58 porosities the permeability could vary by many orders of magnitude, even in similar composition

59 samplesfoams. More than $90 \%$ of these permeabilities are bounded by two empirical power laws,

60 neither of which identifies a percolation threshold.

61 Comparison of the permeability relationships from this study with previous models (Degruyter et al.

62 2010; Burgisser et al. 2017) relating porosity, characteristic pore-throat diameters and tortuosity

63 demonstrated good agreement. However, modifying the Burgisser et al. (2017) model by using the

64 maximum measured pore-throat diameter, instead of the average diameter, as the characteristic

65 diameter improved the fit of theproduced a model that reproduced the lattice-Boltzmann permeabilities

66 to within 1 order of magnitudewith respect to permeability determinations. Measured correlations

67 between the average bubble diameter and the maximum pore-throat diameter as well as between 
68 porosity and tortuosity in our experiments produced relationships that allow application of the modified

69 Burgisser et al. model to predict permeability based only upon the average bubble diameter and

70 porosity. The experimental results are consistent with previous studies suggesting that increasing

71 bubble growth rates result in decreasing permeability of equivalent porosity foams. The effect of

72 growth rate on permeability is hypothesized to substantially contribute to the multiple orders-of-

73 magnitude variations in the permeabilities of natural magmatic foamssamples at similar porosities.

74 Applying the steady-state magma ascent and eruption model of Namiki and Manga (2008), we find that

75 enly magmatic foams with the highest permeabilities will lose their expanding gases, and, therefore,

76 the driving force for their ascent and eruption. The Namiki and Manga (2008) model also indicates that

77 gas loss can oceur at low perosities in highly permeable magmas.

78 Keywords: magmatic foam, permeability, bubble and pore throat sizes, bubble connectivity,

79 synchrotron X-ray tomography

\section{Introduction}

82 The competition between magma expansion due to gas exsolution ef gasand expansion, and gas loss;

83 and the loss of that gas through the porous network created by vesiculation in volcanic conduits, exerts

84 a significant control on the explosivity of volcanic eruptions (e.g., Sparks 2003; Spieler et al. 2004,

85 Mueller et al. 2005, 2008). This competition is profoundly influenced by the permeability of the

86 magmatic foam (a mixture of gas-filled bubbles and melt that may contain crystals and if quenched

87 would produce a scoria or a pumice). Relatively impermeable magmasfoams can lead to violent

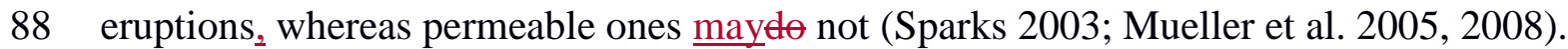

89 Understanding the development of porosity, $\Phi$, and permeability, $\mathrm{k}$, during magma vesiculation is one

90 of the keys to quantitative modeling of volcanic processes that hold the promise of a better 
91 understanding of volcanic eruptions and their precursors (Fagents et al. 2013). Due to the significance

92 of permeability, many studies characterized the porosities and permeabilities of natural samples and

93 experimental run products and demonstrated orders-of-magnitude differences in permeability at similar

94 porosities, finding useful relationships between porosity and permeability (e.g., Klug and Cashman

95 1996; Saar and Manga 1999; Blower 2001; Rust and Cashman 2004; Mueller et al. 2005, 2008; Bouvet

96 de Maisonneuve et al. 2008; Wright et al. 2009; Degruyter et al. 2010; Bai et al. 2010; Polacci et al.

97 2014; Farquharson et al. 2015; Kushnir et al. 2016; Lindoo et al. 2016; Burgisser et al. 2017).

98 The structural details of porous media, such as tortousity and the size of the bubbles and pore throats

99 that connect them have long been known to significantly influence permeability (Carman 1937; Archie

100 1942). Polacci et al. (2008) suggested that "a few large vesicles, exhibiting mostly irregular, tortuous,

101 channel-like textures" in scoria from Stromboli volcano (Italy) were the preferential pathways used for

102 gas escape from the magma. Degruyter et al. (2010) and Burgisser et al. (2017) both demonstrated that

103 the tortuosity of the sample and the characteristic diameter of the pore throats played significant roles

104 in controlling magmatic permeability. These publications demonstrated that the size of the bubbles, the

105 pore throats that connect them, and the ways in which bubbles are interconnected (tortuosity and either

106 in a series or parallel configuration) are significant controls on gas transport in magmatic systems.

107 Using then available measurements, Bai et al. (2010) propesed one general peresity permeability

108 relationship for basaltic foams and another for dacitic-to-rhyolitic ones. Although this compositionat

109 systemization of porosity permeability relationships provides a useful reference, more recent studies of

110 porosity and permeability have indicated that Bai et al.'s (2010) binary division of porosity

11 permeability relationships into basaltic and silicic foams breaks down (e.g., Burgisser et al. 2017).

112 Nevertheless, the permeabilities of basaltic foams are generally greater than those of more silicic foams

113 at similar porosities, suggesting that melt chemistry plays a role in the development of the foam 
structure, and that this structure in turn controls the permeability (e.g., Blower 2001; Wright et al.

115 2009; Bai et al., 2010).

116 This study arose from the hypothesis that foam properties, including the bubble size distribution,

117 tortuosity, the number and size of pore throats, and the degree of interconnectivity between bubbles,

118 play an important role in controlling the permeability of magmatic foams. Here we report results of a

119 series of high-temperature, in situ X-ray tomographic microscopy experiments studying the

120 development of crystal-free, vesiculating samplesfoams of silicate melt at 1 atm. Although in the experiments bubbles are formed during heating at constant pressure (rather than during decompression

122 at approximately constant temperature in volcanic systems), the development of the interconnections

123 between bubbles provides important information on the formation of magmatic foams and development 124 of their permeability. Four melt compositions were studied: a mid-ocean ridge basalt (MORB), a 125 trachyandesite, an andesite, and a dacite. All attempts to study a rhyolitic melt failed due to the 126 inability of the laser furnace used in the experiments (Fife et al. 2012) to heat this composition to

127 temperatures high enough for gas exsolution and bubble formation.

128 The viscous permeabilities of the foams were determined by usingfrom tomographic reconstructions of 129 sample volumes as the input formsing lattice-Boltzmann techniquesimulations of fluid flow (Hill et al.

130 2001; Hill and Koch 2002) with the goal of creating a general model that can be used for the

131 ealculation of silicate foam permeability. We concentrated on the viscous permeability $\mathrm{k}_{1}$, and the

132 applicability of the Carman-Kozeny equation to magmatic foams (Carman 1937). Although we have

133 not investigated the inertial permeabilities, $\mathrm{k}_{2}$, in our samples, relationships between viscous and

134 inertial permeability have been previously determined (e.g., Rust and Cashman 2004; Yokoyama and

135 Takeuchi 2009; Bai et al. 2010; Polacci et al. 2014; Burgisser et al. 2017). Most recently Zhou et al.

136 (2019) proposed a universal power-law equation relating viscous and inertial permeabilities for all 
137 geologic porous media with parameters equivalent to those previously published by Polacci et al.

138 (2014) for volcanic samples. Thus, knowledge of the viscous permeability allows calculation of the

139 inertial permeability using the relationships in Polacci et al. (2014) and Zhou et al. (2019).-Better

140 knowledge of the foam structure holds the promise of increasing our understanding of the relationship

141 between viscous and inertial permeability with the goal of a universally applicable relationship between

142 the two.

143 We show that the viscous permeability of the studied foams is complexly controlled by their structure.

144 We further demonstrate that characterizing the structure of the foam, and particularly the size

145 distribution of pore throat diameters and their cross-sectional areas, is critical to predicting viscous

146 permeabilities to within an order of magnitude. The results are also found to support previous

147 hypotheses concerning the role of bubble growth rate on silieate form permeability. Combining fits to

148 porosity permeability data and a model of magmatic foam ascent demonstrates that only the most

149 permeable samples appear to have the potential to lose the gases that drive their ascent, and that this

50 loss of gas can occur at low porosities.

\section{Methods}

\section{Hydrous glass preparation}

154 Samples of MORB, trachyandesite, andesite, and dacite were chosen for these experiments (Table 1).

155 The MORB is a dredge haul sample graciously donated by C. Langmuir; the trachyandesite is a scoria

156 from the 2010 eruption of Eyjafjallajökull, Iceland, and the andesite and dacite compositions were from

157 Atka Island, Alaska, USA. Each sample was ground to less than $50 \mu \mathrm{m}$ in diameter and dried at $110^{\circ} \mathrm{C}$

158 before use. Approximately $70 \mathrm{mg}$ of powder plus distilled water were loaded into $3 \mathrm{~mm}$ diameter $\mathrm{Pt}$

159 capsules and welded closed in a water bath without volatile loss. Water concentrations are based upon 
160 the water added to the capsules and was $3 \mathrm{wt} \%$ in successful experiments with MORB, trachyandesite 161 and dacite-contained 3 wt \% water. The only successful experiment with the andesite contained $5 \mathrm{wt}$ $162 \%$ water. The rock plus water mixtures were melted above their liquidi in a piston-cylinder apparatus

163 at a temperature of $1250{ }^{\circ} \mathrm{C}$ or $1200{ }^{\circ} \mathrm{C}$ (the trachyandesite only), and a pressure of $1.0 \mathrm{GPa}$ for a

164 duration of $2 \mathrm{~h}$ or of $1 \mathrm{~h}$ (again only the trachyandesite) in $19.1 \mathrm{~mm} \mathrm{NaCl}$-pyrex assemblies (Baker 1652004 ) and quenched isobarically. Subsamples with volumes of approximately $\sim 1$ to $2 \mathrm{~mm}^{3}$ of these 166 crystal-free glasses were used for the synchrotron X-ray tomographic microscopy experiments.

168 In situ synchrotron X-ray tomographic microscopy

169 In situ synchrotron X-ray tomographic microscopy was performed at the TOMCAT beamline of the 170 Swiss Light Source at the Paul Scherrer Institut (Villigen, Switzerland) using a laser-based heating 171 system (Fife et al. 2012) and the ultra-fast endstation (Mokso et al. 2010, see Mokso et al. 2017 for 172 current capabilities of the ultra-fast endstation that differ from the description below and allow more 173 rapid acquisition of images than possible when this study was performed). The laser system comprises 174 two, class four diode lasers of $980 \mathrm{~nm}$ wavelength on opposite sides of, and $40 \mathrm{~mm}$ away from, the 175 sample; these each provide up to $150 \mathrm{~W}$ of power to heat the sample. A pyrometer was used to 176 measure the temperature. The ultra-fast endstation incorporated a pco.DIMAX camera, which acquires 177 and transfers data orders of magnitude faster than traditional CCD technology (Mokso et al. 2010). To 178 reach the highest possible temperature, the lasers were pointed just below the sample holder on the 179 zirconia rod that connected the sample holder to the rotation stage below. The temperature was 180 increased until it reached approximately $600{ }^{\circ} \mathrm{C}$ at which point the sample was lowered into the laser

181 beams. Samples were then heated at either $1^{\circ} \mathrm{C} \mathrm{s}^{-1}$ or $6{ }^{\circ} \mathrm{C} \mathrm{s}^{-1}$ to the maximum temperature of the 182 experiment, resulting in sample vesiculation and creation of a silicate foam under open-system 
183 conditions such that the sample was free to expand and exsolved gas escaped the system. Initially a

184 programmed heating rate of $\sim 6^{\circ} \mathrm{C} \mathrm{s}^{-1}$ was chosen as the best compromise between instantaneous

185 heating of the sample and the need for bubbles to grow slowly enough to be successfully imaged. Due

186 to many experimental failures, a slower programmed heating rate of $\sim 1{ }^{\circ} \mathrm{C} \mathrm{s}^{-1}$ was found to produce

187 more successful experiments (Table 2). Measurement of the time-temperature histories of the

188 experiments demonstrated that the heating rates were often $\sim 20$ percent slower than the programmed

189 ones (Table 2).

190 Experiments were performed by isobaric heating at atmospheric pressure, with a time-temperature-

191 pressure path distinctly different from bubble formation during near isothermal decompression in

192 natural systems, because a high-pressure furnace is not available on the TOMCAT beamline.

193 Subtracting atmospheric pressure from the hydrous melt supersaturation pressure (Table 2) yields the

194 difference between the starting pressure of vesiculation and its final pressure $(0.1 \mathrm{MPa})$ when water

195 was exsolved from the melt. Dividing this pressure drop by the duration of the isobaric heating (Table

196 2) yields equivalent decompression rates of approximately 0.1 to $2 \mathrm{MPas}^{-1}$. Although these equivalent

197 decompression rates are considered only rough approximations for comparison of the experiments with

198 nature, the low values are similar to decompression rates found by Ferguson et al. (2016) for eruptive

199 products of Kilatea voleano and the high values to decompression rates found by Humphreys et at.

200 (2008) for the May 18, 1980 plinian eruption of Mt. St. Helens.

201 Data acquisition was initiated at the first visible onset of vesiculation, such as bubble formation; and 202 sample expansion. During data acquisition, samples reached a maximum temperature between $~ 950$

$203{ }^{\circ} \mathrm{C}$ and $\sim 1200{ }^{\circ} \mathrm{C}$. Polychromatic X-rays were filtered to $5 \%$ power, generating 3 ms exposure times, 204 and 701 projections were captured over an angular range of 180 degrees during continuous rotation.

205 The microscope used for these scans incorporated a specially designed, high numerical aperture 
206 objective lens with itsfour-fold magnification-set to four-fold for these experiments. This corresponded

207 to a $2.89 \mu \mathrm{m} \times 2.89 \mu \mathrm{m}$ pixel size and a $5.83 \mathrm{~mm} \times 5.83 \mathrm{~mm}$ field of view. The optics were coupled to

208 a LuAG:Ce $100 \mu \mathrm{m}$ thick scintillator screen. Reconstructions were performed using a modified

209 GRIDREC algorithm (Dowd et al. 1999; Rivers and Wang 2006; Marone and Stampanoni 2012)

210 coupled with Parzen filtering of the sinograms.

211 Many bubble-growth experiments were performed, but only a few were successful. The most

212 significant problem was image blurring due to sample motion caused by rapid vesiculation and bubble

213 growth that rendered the tomographic reconstructions useless for this study. Other problems were

214 samples that failed to heat to temperatures high enough to vesiculate-(e.g., all rhyolitic samples and

215 some of the other compositions) (which included all rhyolitic samples investigated) and samples that

216 cracked into small pieces during heating.

217 Of the 62 experiments performed, only one dynamic experiment on the andesitic composition, one on

218 the trachyandesitic composition, and one on the dacitic composition yielded 3D reconstructions that

219 could be used to extract quantitative data. Bubble growth in all dynamic experiments on the MORB

220 composition was so rapid and the motion artifacts so severe that no successful reconstructions were

221 made. However, the final steps of 4 experiments on the MORB composition were successfully imaged

222 as bubble growth slowed or stopped due to gas loss from the sample. These experiments span much of

223 the range of porosities measured in the successful tomographic scans on the andesite, trachyandesite,

224 and dacite, and allow comparison between the four different melt compositions. Even the successful

225 experiments contained some image artefacts due to sample movement during bubble growth. These

226 aretefacts were avoided during the sample analysis discussed below.

227

228 Image analysis and quantification 
229 The bubble distributions in the samples were not homogeneous because of thermal gradients in the 230 laser furnace. Thus, only selectedrepresentative central portions of the samples, far from their edges 231 and the capsule walls, were analyzed, and the measurements reported are not representative of the 232 entire sample, but only of the volume investigated. The tomographic reconstructions were inspected 233 with ImageJ and subvolumes from the most vesicular portions of the samples were chosen; in most 234 cases they were $256 \times 256 \times 256$ voxels in volume (Fig. 1a-c). Because these volumes were too large 235 for lattice-Boltzmann determination of their permeability (discussed below) representative subvolumes 236 of $370 \times 370 \times 370 \mu \mathrm{m}^{3}\left(128 \times 128 \times \square 128\right.$ voxels, trachyandesite EFJ-8a), or $462 \times 462 \times 462 \mu \mathrm{m}^{3}$ $237(160 \times 160 \times 160$ voxels, andesite DRB2012-2a, dacite DRB2012-6e-8, -9, -10, MORB DRB2012-7a$2382,-3$, -cf $)$ or $578 \times 578 \times 578 \mu^{3}(200 \times 200 \times 200$ voxels, dacite DRB2012-6e-07, MORB

239 DRB2012-7f-10) were used for all quantitative analyses with the Pore3D software library (Brun et al. 240 2010; Zandomeneghi et al. 2010). An edge-preserving smoothing filter (Tomasi and Manduchi 1998) 241 was applied followed by a 3D, manually selected, global fixed threshold to separate pore space from 242 glass. This segmentation process was adopted for all datasets.

243 The vesicularity was computed as the number of voxels belonging to the pore space with respect to the 244 total number of voxels in the object. Objects, bubbles and pore throats, were not counted unless their 245 size was greater than two voxels, where each voxel was $2.89 \times 2.89 \times 2.89 \mu \mathrm{m}^{3-}$ in volume. Baker et 246 al.'s (2011) study of the reproducibility of porosity measurements using X-ray microtomography

247 Multiple measurements by different investigaters hademonstratedve demenstrated that the precision of 248 vesicularity measurements is approximately 0.01 ; this estimate is based upen multiple $3 \mathrm{D}$ 249 measurements of the same sample in Image J that produced an average porosity of 0.503 with a 250 standard deviation of 0.010 (Baker et al. 2011). We expect similar uncertainties for pore throats 251 because the same techniques were used for measurements of vesicles and pore throats. The 
interconnected porosity was determined from the same images using the ObjectCounter3D plugin in

253 ImageJ (https://imagej.net/3D_Objects_Counter).

254 A family of descriptors based on skeleton analysis (Lindquist and Lee 1996) was applied to derive

255 bubble number and pore throat number density as well as bubble- and pore throat-size distributions. As

256 in our previous research (Baker et al. 2012), the skeletonization algorithm of Brun and Dreossi (2010)

257 was applied.; Eachand_bubble diameters and- the minimum thickness of each pore throat thicknesses

258 wasere computed using the concept of a maximal inscribed sphere, which was moved through the pore

259

260

261

262

263

264

265

266

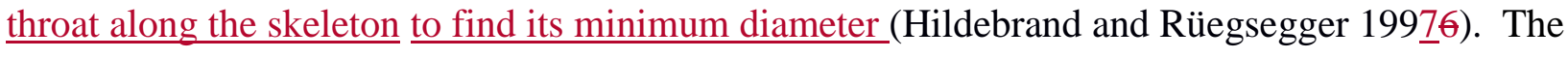
skeletonization algorithm used in this study offers a tuning parameter to control the amount of branches in the output skeleton. Figures $1 d \&$ e present the differences between a case where only the most significant (and larger) interconnects are considered and a case where the smallest interconnects are considered and a skeleton branch is added to the skeleton network for each of them. This parameter is tuned to select the maximum number of branches in this study.

The geometrical determination of pores bubbles and throats is difficult because there is no unambiguous geometrical definition of where a pore bubble ends and a connecting channel begins. Conceptually, the skeleton nodes correspond to pere- bubbles(bubble) bodies, and the branches of the pore-space skeleton correspond to the channels (or paths) connecting the peresbubbles. However, a typical pore/node correction has to be applied because several skeleton nodes may occur in the same pore bubble body (Lindquist 2002), as can be seen in Figures 1d and 1e. In this work, a criterion based on the isotropic inflation of spheres centered on each node was used. If two or more spheres overlap, they are considered part of the same porebubble. The number of identified pores bubbles is actually the number of independent clusters of overlapping inflated bubbles. The amount of inflation can be controlled and acts as a parameter for the merging criterion. The steps in this process are 
275 graphically shown in the example presented in Figures 1d-1h. An insufficient inflation value

276 overestimates the number of pores, while the maximum inflation to fill the entire bubble might

277 underestimate this value if several spurious branches result after skeletonization. In this work a level of

278 inflation equal to $85 \%$ of the maximum was used. Variations around this value were also considered,

279 and it was found that this parameter only weakly affects the computed values of the bubble and pore

280 throat numbers and sizes, as seen in Figures 1f \& $\mathrm{g}$ that presents two examples with different levels of 281 inflation.

282 Some concerns still remain for the throat size determinations; while very short branches are usually

283 disregarded, because some maximal spheres centered at the skeleton nodes may completely enclose the

284 short branches, incorrect channels may still be considered. Practically, this means that the throat-size

285 distribution may present some large-valued outliers due to the consideration of branches that demay not 286 represent physical channels.

287 The final assessment of pore-bubble(bubble) size is based on the diameter of the maximal inscribed sphere placedeentered aten the center of mass of the cluster of the overlapping inflated bubbles (Fig. $1 \mathrm{~h}$

290 identified after skeletonization by the investigated volume. The uncertainties in the numbers of

291 bubbles and of pore throats can be estimated as the square root of the number of each object type

292 counted by application of Poisson statistics.

293 The connectivity, $\beta$, is a standard topological property that measures the number of interconnections (in 294 this study pore throats) between objects (in this case bubbles). Connectivity analysis of tomographic 295 reconstructions was pioneered in studies of bone structure (Odgaard and Dundersen 1993) and of 296 porous media (Thovert et al. 1993). Following these two publications Fo measure $\beta$ we ealculated the 297 first Betti number following Thovert et al. (1993)_and then divided that value by the sample volume to 
298 measurecalculate the connectivity per unit volume $\left(\mathrm{mm}^{3}\right.$ in this study), $\underline{\beta}$ or connective density, as done 299 by Odgaard and Gundersen (1993) using:

$$
\beta=\frac{\# \text { pore throats }-\# \text { bubbles }+1}{\text { sample volume }}
$$

301 This topological measure may yield both negative and positive values per unit volume. As an

302 illustration, consider a simplified example of 4 bubbles in a volume of $0.5 \mathrm{~mm}^{3}$. In the case of no

303 interconnections, or pore throats, between the bubbles, the value of $\beta$ is $-6 \mathrm{~mm}^{-3}$. In cases where only

304 single connections are allowed between bubbles (i.e., two pore throats cannot connect the same two

305 bubbles), a system with two interconnections yields a $\beta$ value of $-2 \mathrm{~mm}^{-3}$, if there are three

306 interconnections $\beta=0 \mathrm{~mm}^{-3}$, and if there are four $\beta=2 \mathrm{~mm}^{-3}$. Thus, a $\beta$ value of $0 \mathrm{~mm}^{-3}$ is the

307 minimum threshold at which the pore throats interconnect the bubbles in this example. This threshold

308 value can exceed 0 if we relax the constraint that bubbles can only be connected by a single pore throat

309 or that all pore throats must interconnect bubbles within the volume of interest. Following basic

$\beta 10$ statistical rules, the uncertainties in the values of $\beta$ are calculated from the uncertainty in the number of

$\beta 11$ bubbles, $\delta$ bubbles, and the uncertainty in the number of pore throats, $\delta$ pore throats, and the sample

312 volume by by

$$
\delta \beta=\frac{\sqrt{(\delta \text { bubbles })^{2}+(\delta \text { pore throats })^{2}}}{\text { sample volume }}
$$

B14 dividing the square root of the sum of the square of the uncertainty in the number of bubbles and the

315 square of the uncertainty in the number of pore throats by the sample volume investigated.

316 Tortuosity in this research is defined as the average distance a particle would travel between two

$\beta 17$ opposite sides of the sample by the pore network divided by the EuclideanEuclidian distance between

318 the opposite sides. The MATLAB® code TORT3D (Al-Raoush and Madhoun 2017) was used for all

319 tortuosity measurements. The tortuosity was determined in three orthogonal directions and then 
320 averaged; the average values are reported in Table 2.

321 The 3D visualization of reconstructed and analyzedanalysed volumes was obtained by the commercial 322 software VGStudio Max 2.0 (Volume Graphics).

\section{Lattice-Boltzmann modeling of permeabilities}

325 Because of the dynamic nature of the experiments and the collapse of the samples with loss of 326 vesicularity near to, or at, their termination, sample permeabilities could not be measured directly.

327 Instead, lattice-Boltzmann modeling of permeabilities was performed using a modified version of an 328 established lattice-Boltzmann code (Hill et al. 2001; Hill and Koch 2002). Details of the permeability 329 modeling applied to tomographic reconstructions can be found in Bai et al. (2010), in which the 330 accuracy of the viscous permeabilities calculated by modeling was directly compared against measured

331 permeabilities and shown to typically be within a factor of 11 for porosities from 0.05 to 0.87 .

\$32 Although, for porosities less than 0.50 the difference between measured and modeled permeability is 333 less than a factor of 7.

334 Bai et al. (2010) demonstrated that the ratio of the tomographic reconstruction resolution (voxel edge 335 length) to the lattice size (edge length of subvolume) and the simulation size could influence the 336 calculated permeability. The resolution-to-lattice-size ratios used in this study are in the range where 337 Bai et al. (2010) demonstrated that the value of the ratio had no effect-of on the calculated permeability. 338 Bai et al. (2010)-studied five samples at different lattice sizes; in three samples they found that 339 decreasing the lattice size below $762 \mu \mathrm{m}$ increased the calculated permeability by a factor of less than

B40 20.- Two of these three samples that display a significant effect of lattice size on calculated

341 permeability contained a giant bubble with a volume of more than $10^{9} \mathrm{\mu m}^{3}$ and more than 3 orders of 342 magnitude larger than the $2^{\text {nd }}$ largest bubbles in the sample (Bai et al. 2008). This largest bubble was 
343 not spherical, but its equivalent radius (i.e., radius of an equivalent volume sphere) was of the same

344 order of magnitude as the recommended lattice size of Bai et al. (2010). We attribute the sensitivity of

345 the calculated permeability in these samples of Bai et al. (2010) to the presence of the large bubble, and

346 note that none of the samples in the present study contain such an anomalously large bubble. The third

347 sample of Bai et al. (2010) that displays a significant lattice size effect does not contain an anomalously

348 large bubble, but has a porosity of only 0.286 . The atAnalysis-of this sample of Bai et al.'s (2010) results

349 indicatessuggests that using the smallest lattice size in this study $(370 \mu \mathrm{m})$ may, at most, overestimate

350 the permeability by a factor of $\sim 3$ for low porosity samples. The other, larger, simulations tested for

351 the effects of lattice size on permeability determinations in Bai et al. (2010) demonstrateused in this

352 study are expected to have uncertainties less than $20 \%$ (Bai et al. 2010). no significant effect of lattice

353 size (i.e., greater than -20 percent). Based upon Bai et al. (2010), we apply an uncertainty of 20

354 percent to the permeability calculations, but recognize that for lower porosity samples the calculated

355 permeabilities may be higher by a factor of 3 .

356 Permeabilities in the three orthogonal directions of each subvolume were calculated. Because the

357 maximum difference between the lattice-Boltzmann permeabilities measured in three orthogonal

358 directions of the samples was less than a factor of 2 , we concluded that there was no significant

359 variation in the permeability as a function of direction and report nosignifieant differences with respect

360 to orientation were detected, the average-of the permeability values for each sample-is reperted in Table

361 2. This lack of orientation effects supports the modeling of our samples as random networks of bubbles

362 interconnected by pore throats.

B64 Bubble growth during isobaric heating versus isothermal decompression

365 Experiments in this study were performed by isobaric heating at atmospheric pressure because a high- 
\$66 pressure furnace was not available on the TOMCAT beamline. The resulting time-temperature-

367 pressure path in these experiments is distinctly different from bubble formation during near-isothermal 368 decompression in natural systems and in many experiments (e.g., Burgisser and Gardner 2004; Lindoo 369 et al 2016, 2017; Mueller et al. 2005; Spieler et al. 2004; Takeuchi et al. 2009).

370 Although bubble nucleation and growth during isothermal decompression and isobaric heating 371 experiments are both driven by supersaturation of the melt with a volatile, the viscosity of the melt 372 increases during isothermal decompression (due to water loss to the bubbles) and may either increase 373 (due to water loss) or decrease (due to increasing temperature) during isobaric heating. For example, in 374 these experiments the andesitic melt begins vesiculation at $900{ }^{\circ} \mathrm{C}$ with a water concentration of $5 \mathrm{wt} \%$ 375 and a viscosity of $\sim 750 \mathrm{~Pa} \mathrm{~s}$ (calculated following Giordano et al. 2008). If this melt lost all its water

376 by the end of the experiment at $1100{ }^{\circ} \mathrm{C}$, the viscosity would be $\sim 6700 \mathrm{~Pa} \mathrm{~s}$. Although it is difficult to 377 estimate the water concentration in the melt during vesiculation, we estimate that approximately $1 \mathrm{wt} \%$ 378 water remains in the melt at $\sim 1000{ }^{\circ} \mathrm{C}$, which would yield a melt viscosity $\sim 3100 \mathrm{~Pa}$ s. During these 379 experiments the diffusivity of water in the melt also is controlled by a combination of heating and 380 dehydration. Using the equations of Ni and Zhang (2018), the water diffusivity at the start of 381 vesiculation is expected to be $\sim 3 \times 10^{-13} \mathrm{~m}^{2} \mathrm{~s}^{-1}$, decreasing to $\sim 1 \times 10^{-14} \mathrm{~m}^{2} \mathrm{~s}^{-1}$ when water is lost. On 382 the contrary, for isothermal decompression at $1100^{\circ} \mathrm{C}$ of the same melt composition, the viscosity 383 would increase from $32 \mathrm{~Pa} \mathrm{~s}$ at the start of vesiculation to $\sim 6700 \mathrm{~Pa}$ s if all of the water is exsolved 384 from the melt, and the water diffusivity would decrease from $\sim 3 \times 10^{-12} \mathrm{~m}^{2} \mathrm{~s}^{-1}$ to $\sim 1 \times 10^{-14} \mathrm{~m}^{2} \mathrm{~s}^{-1}$. 385 These differences in the history of the melt viscosity and diffusivity will influence the rates of bubble 386 growth and coalescence of neighboring bubbles. Following Navon and Lyakhovsky (1998) the radius 387 of an individual bubble during its initial stages of growth will be significantly affected by the melt 388 viscosity: 
390 where $\mathrm{r}$ is the bubble radius, $\Delta \mathrm{P}$ is the supersaturation pressure, $\eta$ is the melt viscosity, and $\mathrm{t}$ is the time.

391 As the bubble grows and supersaturation decreases, the bubble radius is described by a law containing

392 the square root of the product of the volatile diffusivity in the melt and time (Equation 36 of Navon and

393 Lyakhovsky 1998). Melt viscosity also exerts control on the time necessary for interacting bubble

394 walls to fail and coalescence to begin, $\tau_{\mathrm{df}}$ :

395

$$
\tau_{d f}=\frac{3 \eta r^{2}}{4 \Delta P h_{\min }^{2}}
$$

396 where $\mathrm{h}_{\min }$ is the critical thickness at which the walls fail (Navon and Lyakhovsky 1998). Equations 3

397 and 4 demonstrate that the rates of bubble growth and coalescence during the early stages of isobaric

398 heating experiments should be slower than those in isothermal decompression experiments because of

399 the higher viscosities and lower water diffusivities in the melt early in the isobaric heating experiments.

400 However, as both types of experiments reach the end of bubble growth and water loss, the rates at

401 which bubble growth and coalescence occur should converge.

402 Both isothermal decompression and isobaric heating experiments are expected to produce similar,

403 random bubble, or foam, structures, due to the stochasticity in both the location and timing of bubble

404 nucleation in the melts. Because of the higher viscosities and lower diffusivities of isobaric heating

405 experiments, the vesicularity and interconnectivity of bubbles (due to coalescence) may be expected to

406 be smaller than in isothermal decompression experiments of similar, short durations (Equations 3 and

407 4). Because of these differences between isothermal decompression and isobaric heating experiments

408 we did not study bubble growth and coalescence rates, but instead concentrated on the development of

409 porosity and permeability of the foams, while fully recognizing that the values we determined may be

410 minimal ones. 
411 Despite the differences between isothermal decompression and isobaric heating experiments, we can

412 make a comparison between the rates of magma ascent and the rates of degassing during isobaric

413 heating experiments by dividing the melt supersaturation pressure (Table 2) by the duration of the

414 isobaric heating (Table 2) to yield equivalent decompression rates of approximately 0.1 to $2 \mathrm{MPa} \mathrm{s}^{-1}$.

415 Although these equivalent decompression rates are only rough approximations, the low values are

416 similar to decompression rates found by Ferguson et al. (2016) for eruptive products of Kilauea

417 volcano and the high values to decompression rates found by Humphreys et al. (2008) for the May 18,

4181980 plinian eruption of Mt. St. Helens.

420 Results

421 Visual observations

422 Bubble growth in the samples occurred rapidly at temperatures above $600{ }^{\circ} \mathrm{C}$. Typical bubble 423 nucleation and growth can be seen in Figure 2 and Supplemental Movie 1 of andesitic sample 424 DRB2012-2a, which was heated from $600{ }^{\circ} \mathrm{C}$ to $1100{ }^{\circ} \mathrm{C}$ over a period of $1040 \mathrm{~s}$ (Table 2). No 425 evidence of a significant nucleation delay (i.e., longer than a few seconds) was detected in any sample.

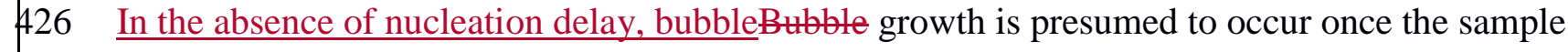

427 temperature exceeds that of the glass transition. The onset of the glass transition temperatures for the 428 samples studied can be estimated using the results of Giordano et al. (2005). The glass transition given 429 in Giordano et al. (2005) for basaltic, trachytic and dacitic compositions determined at their most rapid 430 heating rate of $0.333 \mathrm{~K} \mathrm{~s}^{-1}$ and water concentrations up to $2.5 \mathrm{wt} . \%$ were fit by a power-law, which was

431 chosen because power-laws describe the effect of water addition on melt viscosity (e.g., Shaw 1972).

432 The power-law fit reproduces the calibrating data to within a maximum of $\sim 50 \mathrm{~K}$, as well as

433 reproducing the anhydrous andesite glass transition temperature of Neuville et al. (1993) that was not 
434 used in the calibration. We estimate a minimum onset of the glass transition in our samples with $3 \mathrm{wt} \%$ 435 water (MORB, trachyandesite, and dacite) at $460{ }^{\circ} \mathrm{C}$ and in the andesite with $5 \mathrm{wt} \%$ water at $440{ }^{\circ} \mathrm{C}$.

436 However our heating rates were approximately 3 to 15 times more rapid than used in Giordano et al.'s 437 experiments, thus the observed vesiculation temperatures were significantly above these minimum 438 glass transition temperatures, in the range of 616 to $900{ }^{\circ} \mathrm{C}$ (Table 2). Although these delays may be attributable to a short nucleation delay, we interpret them as being due to our inability to observe the onset of bubble growth in real time during the experiments because of the small size of the initial

441 bubbles. This inability to see the earliest bubbles made it difficult to measure samples at low porosities 442 and only a few data at these conditions were obtained (Table 2).

443 Bubble growth was initially observed as a dense cloud of small bubbles that grew into larger, easily 444 discernible, bubbles that rapidly became-an interconnected foam-(Fig. 2). Typically, these early growth 445 rates were so rapid that they were blurred in the tomographic images, so meaningful quantitative 446 measurements could not be made.

447 Visual inspection of the tomographic reconstructions revealed that early bubbles vary from ellipsoidal 448 to sub-spherical, but within seconds all evolve into sub-spherical to spherical shapes. Bubble growth in 449 all experiments on the basaltic composition was too rapid to quantitatively analyze those samples 450 before termination of the experiment. However, in four cases we extracted limited data from the 451 different stages of foam development (Table 2). Bubbles coalesced and typically grew to a maximum 452 size, creating a foam withef thin-walled bubbles. If the sample was not immediately quenched, the 453 foam contracted and underwent partial collapse, and in some cases collapse occurred before the 454 termination of the experiment. This behavior is attributed to the loss of volatiles, either through failure 455 of the bubble walls or diffusion through them.

456 Not every tomographic reconstruction set could be used for all the quantitative analyses presented in 
457 the Methods section. In some cases, permeabilities could not be determined (e.g., DRB2012-6e-07)

458 because of the large computer memory required to resolve the surface area of the interconnected

459 bubbles.

461 Bubble number density (BND), bubble size distribution (BSD), and pore throat size distribution (PTD)

462 Table 2 presents a summary of our quantitative measurements, and the sizes of all bubbles and pore

463 throats measured in the studied samples are provided as supplementary material (Table S1). All

464 measurements reported in this study were made on volumes far from the edges of the samples and that

465 displayed no visual evidence of anisotropy. The typical distance of the analyzed volume from the

466 sample edges was approximately $500 \mu \mathrm{m}$. Because of the small sample volume, in some cases the

467 number of bubbles and pore throats, as well as their range in size, are small (Table S1). In all but one

468 case for the dacitic composition, DRB2012-6e-10, the largest bubble in the investigated volume was

469 less than $6 \%$ of the sample volume. In DRB32012-6e-10 the largest bubble was $31 \%$ of the volume.

470 In general, in each sample theThe bubble number densities in the foams tend to increase with porosity

471 up to a maximum and decrease at higher porosity (Figs. 3-6, Table 2), although between any two steps

472 the BND can decrease (Table 2). Increasing BNDs are attributed to continuous nucleation and

473 decreasing BNDs to bubble coalescence. The continuous nucleation is notable because many of these

474 experiments were only slightly supersaturated with water by $35 \mathrm{MPa}$, although the andesite experiment

475 was supersaturated by $168 \mathrm{MPa}$ (Table 2). The basaltic foams reached the highest BND of $1.92 \times 10^{4}$

$476 \mathrm{~mm}^{-3}$, whereas the dacite had the lowest BND of only $4.01 \times 10^{3} \mathrm{~mm}^{-3}$ in its final step.

477 The measured bubble size and pore throat size distributions of the experimentsal foams are presented in

478 Figures 3-6. Each figure presents a volume-normalized histogram of the sizes of either the bubbles or

479 the pore throats. None of the distributions are Gaussian, but instead often display "long tails" to large 
bubble and throat sizes, a characteristic indicative of power-law distributions (Newman 2005).

481 Although the bubble-size and throat-size distributions can be fit with power-laws, this was not done in 482 this study due to the small size range-of sizes_in each distribution that results in large uncertainties in 483 the calculated power-law exponent. Despite evidence that the sizes are power-law distributed, and 484 therefore their means and standard deviations do not have the same significance as in Gaussian 485 distributions (Newman 2005), each panel of each figure provides the mean diameter and the standard 486 deviation of either the bubble diameter or pore throat diameter to allow comparison of the 487 measurements with previous studies (e.g., Burgisser et al. 2017). The relative standard deviations 488 about the mean bubble sizes and pore throats are in most samples greater than $25 \%$ and often near $50 \%$. 489 These large standard deviations are consistent with the non-Gaussian distributions of bubble and pore 490 throat sizes (Newman 2005).

491 Bubbles measured in the basaltic composition reach a size of approximatelylmest $46200 \mu \mathrm{m}$ in 492 diameter, and the BNDs are in the thousands per cubic millimeter (Fig. 3a, Table 2). The two samples 493 with the lowest porosities $(0.52$ and 0.50$)$ are from the same experiment and demonstrate that with time 494 the number of small bubbles in the 5 to $10 \mu \mathrm{m}$ range increases, which indicates continuing bubble 495 nucleation and growth, as does that of larger bubbles in the 40 to $70 \mu \mathrm{m}$ range, which provides 496 evidence of continued growth. The disappearance of bubbles in the size range of 55 to $60 \mu \mathrm{m}$ of the 497 second sample $(\Phi=0.50)$ is interpreted as a consequence of bubble coalescence, and its lower porosity 498 is probably due to bubble collapse that occurred between these two heating experiments (Table 2).

499 Comparison of the average bubble size in each experiment does not indicate any significant trend with 500 porosity (Fig. 3a).

501 The number of pore throats in the basalt varies from about $6.40 \times 10^{3} \mathrm{~mm}^{-3}$ at the lowest porosity to 502 approximately $7.22 \times 10^{4} \mathrm{~mm}^{-3}$ at the highest porosity. The two low-porosity $(0.50$ and 0.52$)$ 
503 measurements of pore throats from the same experiment demonstrate only moderate increases in the 504 number and size of pore throats (Fig. 3b, Table 2). The experiment with 0.55 porosity contains one 505 pore throat that is anomalously larger then the next largest one (Fig. 3b), and our experimental

506 techniques for measuring the pore diameter may produce large-valued outliers in the pore throat size 507 distributions. Whereas we cannot completely discard the possibility that the largest pore throat in this

508 sample is an artefact, we think that size difference between the largest pore throat and the others in the 509 distribution (Fig. 3b) is not sufficiently large to discard the measurement. _However, growth in the 510 number of smaller pore threats and loss of pore throats in the 45 to $50 \mu \mathrm{m}$ size range are evident.

511 Because the basaltic results for the two highest porosity measurements $(0.55$ and 0.73$)$ are from

512 different experiments, it is difficult to investigate the evolution of BSD and PTD with increasing

$\$ 13$ porosity. Nevertheless, if these two samples are interpreted as an evolutionary trend, there appears to

514 be a trend of an increasing number of pore throats with increasing porosity (Fig 3b, Table 2).

516 In contrast to the basaltic experiments, all of the andesite data (Fig. 4a, b, c, Table 2) were collected

517 from the same experiment; however the location of the sample volume quantified varied slightly

$\$ 18$ between different time steps in order to choose the most representative volumes near the center of the

519 samples and to avoid volumes containing motion artefacts and/oreeunting anomalously large or small

520 bubbles. The maximum bubble size was between 215 and $220 \mu \mathrm{m}$ (Fig. 4c). All andesite BSDs are

$\$ 21$ dominated by bubbles in the 5 to $20 \mu \mathrm{m}$ size range; consequently between porosities of 0.17 to 0.59 the 522 average bubble diameter only increases from 10.1 to $13.6 \mu \mathrm{m}$, and only reaches a maximum of $21.5 \mu \mathrm{m}$ 523 in the last experiment_(Fig. 4a, b, c). The large number of small bubbles in all but the final porosity is 524 interpreted to reflect a process of continuing nucleation and growth of new bubbles. The appearance of 525 larger bubbles, greater than $40 \mu \mathrm{m}$, at porosities above 0.41 is caused by the expansion of gas within 
them during heating, and by coalescence. Evidence of coalescence is seen in the decreasing number of

527 intermediate-sized bubbles with the appearance of larger bubbles in the highest porosity samples (cf.,

$\$ 28$ Gaonac'h et al. 1996).

_The PTD distributions demonstrate the growth of throat sizes and number with increasing porosity

530 (Figs. 4d, e, f). Because the number of pore throats is dominated by smaller ones, the average throat

531 diameter only increases from 8.0 to $10.6 \mu \mathrm{m}$ in the experiment; however $\mathrm{t}$ The largest pore - pore throat

532 size grows from 10-15 $\mu \mathrm{m}$ to a maximum of 50-55 $\mu \mathrm{m}$. In comparison to the basalt, there are fewer

533 pore throats greater than $30 \mu \mathrm{m}$.

534 The BSDs for the trachyandesite at the two highest porosities display broad peaks in the distribution

535 without long tails to large bubble sizes seen in some of the andesite and basalt BSDs (Fig. 5a, Table 2).

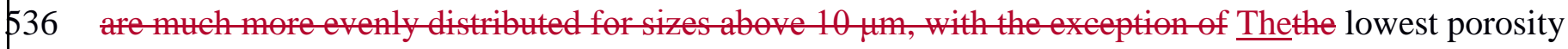

537 sample hadwith only a single bubble and no pore throats in a volume of $0.0506 \mathrm{~mm}^{3}$ (Fig. 5a, b, Table

538 2). The average bubble size grows from 8.2 to $46.2 \mu \mathrm{m}$ with increasing porosity, and $\mathrm{t}$ The maximum

539 bubble measured was in the 95 to $100 \mu \mathrm{m}$ size class. Although no bubbles less than $10 \mu \mathrm{m}$ were

$\$ 40$ observed at a porosity of 0.61 , one appears at $\Phi=0.64$, suggestingproviding evidence of continuing

541 bubble nucleation. Bubble coalescence between the same two porosities is indicated by the decreasing

$\$ 42$ number of bubbles with sizes between 10 and $75 \mu \mathrm{m}$ and increasing numbers of larger-sized bubbles in

543 the same sized volume (Fig. 5a).

544 _ The PTDs of the trachyandesite show a growth in the density of pore throats between 0.30 and 0.61

porosity followed by a decline in densities between 0.61 and 0.64 porosity with an increase in the

$\$ 46$ number of larger pore throats (Fig. 5b.). The average size of the pore throats are similar to the other

547 eompositions studied, and only vary from 8.1 to $10.3 \mu \mathrm{m}$.

548 The dacite BSDs demonstrate the growth of larger bubbles with increasing porosity, up to a maximum 
of almost $400 \mu \mathrm{m}$ at $\Phi=0.84$ (Fig. 6a). The change in the BSDs with poresity results in the average 553 1996).

554 The PTDs of the dacitic sample demonstrate growth of larger pore throats up to a porosity of 0.87 555 556

bubble diameter increasing from $11.4 \mu \mathrm{m}$ to $25.9 \mu \mathrm{m}$ at the highest porosity. Bubbles in the size range of $10-15 \mu \mathrm{m}$ increase in number between porosities of 0.38 and 0.79 , and subsequently decrease as larger bubbles appear at higher porosities, a behavior we attribute to coalescence (cf., Gaonac'h et al. followed by a decrease in that value as the sample porosity at the end of the experiment toreached $\Phi=$ 0.84 (Fig 6b). Although the largest pore throats reach $70 \mu \mathrm{m}$, the distributions are dominated by smaller pore throats that result in average pore throat diameters only increasing from 7.9 to $10.9 \mu \mathrm{m}$ with growing poresity. The loss of the large pore throats seen in the distribution for $\Phi=0.84$ is associated with the growth of the large bubbles in this sample (Fig. 6a), which are presumed to have incorporated the larger pore throats into them during growth.

\section{Connectivity, coordination number, and tortuosity}

In all cases, the connected porosity is similar to the total porosity (Table 2); however the connectivity, $\beta$ (Eqn. 1), varies significantly (Table 2, Supplementary Fig. 1). The $\beta$ values in each type of foam increase from values in the hundreds to thousands per cubic millimeter with increasing porosity (Table 2) up to maximums in the tens to hundreds of thousands per cubic millimeter and then, with the exception of the basaltic composition, decrease at higher porosities (Table 2). This trend is similar to those observed for the BNDs and the PTDs (Table 2, Figs. 3-6).

The average coordination number (or number of interconnected bubbles surrounding a specified bubble) for each porosity (Table 2) of an individual melt composition is similar and varies between $\sim 4$ and 6, with a few outliers reaching values near 7 (DRB2012-7c-f, DRB2012-2a-18, DRB2012-6e-10) 
572 and one almost reaching 8 (DRB2012-2a-19). These average values are far below the maximum value

573 of equal-volume, deformable bubbles surrounding a central bubble (the kissing number) of 32 (Cox and

574 Graner 2004). However, the standard deviations about the average for each sample are often as large

575 as, or even larger than, the mean, and the maximum coordination numbers are often near 100 and can

576 reach almost 600 (Table 2). The bubbles with such high coordination numbers are large and

577 surrounded by a network of small bubbles. (A two-dimensional image of such a bubble can be seen in

578 Fig. 1.) Such high values of the kissing number are not inconsistent with simulations of polydisperse

579 foams that display average coordination numbers between 11 and 14, but contain some foam polyhedra

580 with coordinations approaching 100 (Kraynik et al. 2004).

581 The tortuosity, $\tau$, of the foams varies from a low of 1.09 (dacites DRB2012-06e-8 and DRB2012-6e-9)

582 to a high of 1.72 (andesite DRB2012-2a-9); however, most tortuosity values fall between 1.1 and 1.3

583 (Table 2). The relationship between increasing porosity and decreasing tortuosity in the studied

584 samples can be described by (cf., Wright et al. 2009, Degruyter et al. 2010):

585

586

587

588

589

590

\section{Permeability}

592 The lattice-Boltzmann (LB), viscous permeabilities, $\mathrm{k}_{1}$, of the samples vary from $3 \times 10^{-15}$ to greater 593 than $5 \times 10^{-11} \mathrm{~m}^{2}$ (Table 2, Fig. 7). However, ourNote that simulations failed-for twoone samples, 
594 (DRB2012-6e-7,EFJ-8a-06), failed due to its extraordinarily fine structure, andso theirits permeability

could not be determined using lattice-Boltzmann simulations (discussed above). Another sample, EFJ08a-06, had only one bubble and its permeability also could not be determined.

The data sets in Figure 7 were fit with a power law because both the Carman-Kozeny relation (Carman 1937) and percolation theory (Stauffer and Aharony, 1994) predict a power-law relationship between porosity and permeability. Formally, the percolation theory relationship is expected to be $k_{1} \sim$ $\left(\Phi-\Phi_{c}\right)^{\mu}$, where, $k_{l}$ is the viscous permeability, $\Phi_{c}$ is the critical porosity threshold where the sample becomes permeable, and $\mu$ is an exponent that depends upon the system dimensionality (Stauffer and Aharony, 1994). The critical porosity threshold for monodisperse spheres in three dimensions is $\sim 0.29$ (Domb 1972; Lorenz and Ziff 2001); however, as discussed in detail below, the critical porosity threshold is a function of the size distribution and shape of the vesicles. Permeability thresholds in natural and experimental magmatic foams can vary from below 0.03 (e.g., Saar and Manga 1999; Bai et al. 2010) to values in excess of 0.63 (Takeuchi et al. 2008, 2009; Lindoo et al. 2016). The critical porosity threshold for any given sample is unknown, and the porosity-permeability relationships in some magmatic foams have been empirically fit with a power law of the form $k_{1}=\mathrm{A} \Phi^{B}$, where $\mathrm{A}$ and B are fitting constants (e.g., Klug and Cashman 1996; Bai et al. 2010), although in some other studieseases the estimated critical porosity threshold (typically 0.3 ) has been included in permeabilityporosity relationships (e.g., Saar and Manga 1999; Blower 2001; Rust and Cashman 2004, 2011).

Our most complete data set on the andesitic composition foam displays a power-law relationship between porosity, $\Phi$, and LB permeability of $k_{1}=1.68 \times 10^{-11} \Phi^{5}$ (Fig. 8). Assuming a critical porosity of 0.010 below the minimum porosity at which a LB permeability was determinedmeasured, or 0.15 , produces a percolation theory power law fit of $k_{1}=5.79 \times 10^{-12}\left(\Phi-\Phi_{c}\right)^{2.1}$. At porosities up to $\sim 0.60$, the measurements are similar to the Bai et al. (2010) fit to permeabilities of silicic rocks 
617 measured by Klug and Cashman (1996). The LB permeabilities of andesitic foams at porosities greater

618 than $~ 0.60$ diverge from Bai et al.'s (2010) fit to silicic foams leading to an order of magnitude

619 difference between the two at a porosity of 0.90 (Fig. 7).

620 The LB permeabilitiespermeability of two basaltic foams at $\sim 0.50$ porosity-_bracket the fit to the 621 andesitic foam but the LB permeability increases to a value that is an order of magnitude greater at a 622 porosity of $\sim 0.55$. The permeability values at $\sim 0.50$ porosity (DRB2012-07a-3) may be artificially low

623 because of bubble bursting that was observed at the end of previous experiment (DRB2012-07a-2), and 624 some of the porosity and pore throats may have been lost. These measurements at 0.50 and 0.55

625 porosity were made on two different chips of basaltic melt; this complicates the interpretation because

626 the two samples have slightly different time-temperature histories, which together with the bubble

627 popping in the sample at 0.50 porosity may might create differences in foam porosity and permeability.

628 Thus, we doubt that the factor of ten increase in permeability between $\sim 0.50$ and 0.55 porosity for

629 these basaltic samples is significant. The LB permeabilities at 0.55 and 0.73 porosity are similar to

630 those found by Bai et al. (2010) on a high-K composition basaltic foam from Stromboli, as shown by

631 the fit to their data in Figure 7.

632 The trachyandesitic foam displays a unique behavior. At low porosities, near 0.30, its LB permeability 633 is significantly above that of the andesiteic experiment, but at higher porosities the LB permeability 634 falls to values similar to the andesitic composition (Fig. 7).

635 The LB permeability of the dacitic composition at 0.79 porosity cannot be distinguished from that of 636 the similarly porous andesiteic experiment, but the two higher porosity dacitic foams surprisingly 637 display significantly higher LB permeabilities than expected from the trend described by the lower 638 porosity dacitic and andesitic formexperimentss (Fig. 7).

639 An obvious and significant difference in the physical properties of the studied compositions is their 
viscosity (Table 1). However, a simple correlation between melt viscosity and permeability at a

641 specific porosity does not exist. The lack of such a correlation is illustrated by the permeability

642 determinations of the trachyandesitic foam at 0.35 porosity and the two highest permeabilities of the

643 dacitic foams (Fig. 7). Although the viscosities of the melts that form these trachyandesitic foams,

644 either with their initial water concentration or anhydrous (Table 1), are at least an order of magnitude

645 higher than basaltic melts, their permeabilities are similar to those of basaltic foams. Comparison of

646 melt viscosities with the average pore-throat diameters in the foams (Figs. 3-6) does not provide clear

647 evidence of a positive correlation between these two properties (cf., Polacci et al. 2014).

648 The experimental results demonstrate that both average bubble sizes and pore throat sizes increase with

649 increasing vesicularity; however, the increase in pore throat sizes is less than that of the bubbles (Figs.

650 3-6). The maximum bubble sizes are observed in the dacitic composition, reaching nearly $400 \mu \mathrm{m}$ in

651 diameter, whereas the maximum bubble sizes for the basaltic, andesitic, and trachyandesitic

652 compositions are 180,220 , and $100 \mu \mathrm{m}$, respectively. The maximum throat diameters are near $100 \mu \mathrm{m}$

653 in the basaltic foam, but only $55 \mu \mathrm{m}$ in the andesitic foam, $35 \mu \mathrm{m}$ in the trachyandesitic foam, and 65 in

654 the dacitic foam. Increasing vesicularity increases connectivity and decreases tortuosity. All of these

655 changes in foam structure result in higher permeabilities that are not simply related to the melt

656 compositions investigated.

\section{Discussion}

659 Permeability is not a simple function of porosity

660 Porosity is often considered the primary control of permeability; in most cases increasing porosity

661 results in higher permeabilities (e.g., Fig. 7), but it has long been understood that other variables such

662 as bubble sizes, composition, connectivity, pore throat diameter, tortuosity etc. significantly influence 
663 permeability (e.g., Carman 1937; Archie 1942; Rust and Cashman 2004; Mueller et al. 2005; Bai et al.

664 2010; Degruyter et al. 2010; Polacci et al. 2012, 2014; Lindoo et al. 2016; Burgisser et al. 2017;

665 Colombier et al. 2017). Our results demonstrate that at $\sim 0.5$ poresity basalts can have permeabilities

666 similar to andesites, and at porosities approaching 0.9 the permeabilities of basalts and dacites are

667 similar (Fig. 7).

668 An obvious and significant difference in the physical properties of the studied melt compositions is

669 their viscosity (Table 1). However, a simple correlation between melt viscosity and permeability at a

670 specific porosity does not exist. The experimental results using crystal-free samples demonstrate that

671 at $\sim 0.5$ porosity basalts can have LB permeabilities similar to andesites, and at porosities approaching

6720.9 the lattice-Boltzmann permeabilities of basalts and dacites are similar (Fig. 7) despite these melts

673 displaying orders-of-magnitude differences in their viscosities (Table 1). Comparison of melt

674 viscosities with the average pore-throat diameters (Figs. 3-6) does not provide clear evidence of a

675 positive correlation between these two properties either (cf., Polacci et al. 2014).

676 Thus, enur study provides no evidence that composition alone controls the permeability-porosity

677 relationship. Connectivity also does not provide a simple predictor of permeability, as evidenced by an

678 order of magnitude difference in the lattice-Boltzmann permeability of samples with similar

679 connectivity and porosity (Table 2, Fig. 7, Supplementary Figure 1). Tortuosity also does not appear to

680 directly correlate with the porosity-permeability relations of the foamssamples studied (Table 2).

681 However, the bubble- and pore throat-size distributions (Figs. 3 - 6) suggest that larger-pores- bubbles

682 and larger pore throats play a significant role in influencing the permeabilities of magmatic foams.

683 Before quantitatively investigating the role of bubble- and pore throat sizes in controlling the

684 permeability of magmatic foams, we_firstcompare our results with those from previous studies. 
687 Our lattice-Boltzmann permeability determinations are compared with-many previous measurements of 688 porosity and permeability from aphyric to low-crystallinity natural and experimental samples in Figure 8. These dataplotted in Figure 8 were taken from published studies to illustrate the distribution of measured permeabilities and porosities. The lattice-Boltzmann permeability determinations of the experiments in this study are consistent with previous measurements of similar composition samples

692 (Fig. 8), even though in some cases our experimental samples are orders of magnitude smaller than 693 natural samples. At any given porosity the combined data set demonstrates that permeability can vary 694 by orders of magnitude; nevertheless, the data define significant trends as porosity increases from 695 negligible values up-to near 1.0 (Fig. 8).

696 At low porosities, the few permeability determinations in the region of 0.02 to 0.18 porosity show no

697 evidence of significant compositional dependence at equivalent porosity (Fig. 8). - Most viscous 698 permeabilities increase from $\sim 10^{-17} \mathrm{~m}^{2}$ at porosities near 0.01 to $\sim 10^{-13} \mathrm{~m}^{2}$ at 0.20 to 0.30 porosity, 699 although some rhyolites at these porosities have permeabilities of only $10^{-15} \mathrm{~m}^{2}$ (Fig. 8). At porosities 700 above $\sim 0.3$, the sphere percolation threshold (Lorenz and Ziff 2001), the permeabilities continue to 701 increase but at a slower rate than observed at lower porosities (Fig. 8). For porosities between 0.5 and 7020.9 , the permeabilities in Figure 8 range from values as low as $\sim 10^{-14} \mathrm{~m}^{2}$ (Lindoo et al. 2016) to as 703 high as $10^{-10} \mathrm{~m}^{2}$ (Bai et al. 2010). The slow increase in permeability at porosities above 0.3 suggests 704 that once a permeable pathway is created, the addition of other pathways for gas transport at higher 705 porosities (as shown by higher values of $\beta$ and lower values of tortuosity) increases the permeability 706 much less significantly than the first pathway. Most lattice-Boltzmann permeabilities measured in this 707 study are near the center of the trend in Figure 8, with the exceptions of the highest permeability

708 basaltic and dacitic melts.; and some basaltic samples with only a few percent porosity have 
709 permeabilities between $10^{-14}$ and $10^{-12} \mathrm{~m}^{2}$ (Fig. 8).

710 At porosities from 0.18 to 0.30 the permeability porosity curves begin to flatten (Fig. 8). The extensive

711 data sets of Farquharson et al. (2015) and Kushnir et al. (2016) on andesitic compositions create a sub-

712 horizontal swath in Figure 8, but are consistent with a trend of increasing permeability for poresities

713 from 0.18 to 0.30 . Interestingly, these two studies of andesite each demonstrate almost a two order-of

714 magnitude variation in permeability at similar porosities (Fig. 8). Our permeability determinations on

715 the andesitic melt show an order-of-magnitude increase in permeability from 0.17 to 0.33 porosity.

716 The trachyandesitic sample with 0.35 poresity has a permeability close to the value predicted by the

717 Bai et al. (2010) basalt line (Fig. 7). The observation that this foam containing 58 wt. \% SiOz has a

718 permeability more similar to basaltic compositions than other foams with similar compesitions (Table

719 1) is inconsistent with a model that simply uses melt composition or melt viscosity to explain the

720 porosity permeability relationship (e.g., Bai et al. 2010). The trachyandesitic foam with 0.35 poresity

721 is notable for its high bubble number density and pore throat density, which appears to account for its

722 high permeability (Fig. 5a, b).

723 In general, silicic foams have lower permeabilities and mafic foams higher permeabilities, but the

724 dacitic foams with greater than 0.80 porosity measured in this study have lattice-Boltzmann

725 permeabilities similar to basaltic foams with similar porosities (Saar and Manga 1999; Bai et al. 2010).

726 Rust and Cashman's (2004) permeabilities of rhyolite, pumice and obsidian as well as Farquharson et

727 al.'s (2015) permeabilities of pumiceous andesite demonstrate almost a two order-of-magnitude

728 variation at similar porosities (Fig. 8). Thus the influence of composition on any porosity permeability

729 relationship appears to be weak.

730 The porosity-permeability data set contains many determinations at porosities below the nominal

731 critical value for mono-disperse spheres. Each specific sample may have its own critical porosity based 
732 upon the size distribution and shape of the bubbles and pore throats. Because of our lack of knowledge

733 of the critical porosity for each sample, the data were fit by empirical power laws without including a

734 critical porosity. Although the data in Figure 8 can be empirically fit with a single power law, $k_{1}=$

$7356.0 \times 10^{-12} \Phi^{4.0}$, the dispersion of the permeabilities around this average fit is orders of magnitude at

porosities from 0.15 to 0.90 (Fig. 8). The range of permeabilities displayed in Figure 8 also can be

737 bound by two power-law fits. The lower bound is constrained by permeability measurements below

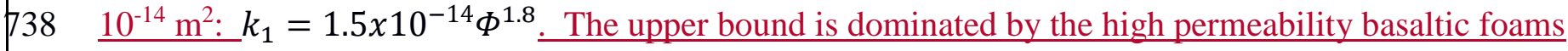

739 of Saar and Manga (1999) and of Bai et al. (2010), and can be described by a power law of $k_{1}=$

$7401.5 \times 10^{-10} \Phi^{4.0}$. More than $90 \%$ of the viscous permeability determinations in Figure 8, including

741 those of this study, fall within the boundaries defined by these two power-laws.

742 Percolation theory can explain Tthe great increase in permeability between 0 and 0.30 porosity.seen

743 for most permeability determinations can be explained by percelation theory. This theory predicts that

744 at about 0.29 porosity an ensemble of inter-penetrating, equivalent diameter spheres that are randomly

745 distributed in three-dimensional space will create a spanning, permeable network of connected porosity

746 (Domb 1972; Lorenz and Ziff 2001). The permeability at porosities below 0.3 and the lack of

747 permeability in some foams at porosities as high as $\sim 0.86$ (e.g., Takeuchi et al. 2008, 2009; Lindoo et

748 al. 2016; Burgisser et al. 2017) mayean_reflect the observations that statistical nature of the percolation

749 threshold in finite systems, which does not necessarily occur at a specified porosity for a non-infinite

750 system (Stauffer and Aharony 1994; Colombier et al. 2017). Possibly the permeabilities at low

751 porosities are controlled by the percolation threshold for hard spheres, which has been determined at a

752 porosity of 0.1938 (Ogata et al. 2005) or 0.1990 (Ziff and Torquato 2017). Another possibility is that a

753 non-constant size distribution of bubbles will affect the percolation threshold. However, $\underline{\text { Consiglio et }}$

754 al. (2003) Ogata et al. (2005) and Ogata et al. (2005) demonstrated that the effect of the non-uniform 
755 765 threshold.

size distributions they investigated on the percolation threshold was not significant for interpenetrating spheres and hard spheres, respectively. demonstrate that for either a uniform size distribution or a lognormal distribution the effect of size is minor and only decreases the percolation threshold of hard spheres by far less than one percent of the porosity for the size distributions they studied. Consiglio et al. (2003) investigated the percolation of inter penetrating spheres of two different sizes and found that by varying the ratio of the two different-sized spheres the porosity at which percolation occurred could increase by less than 0.01 when compared to the results for spheres of a single size. Thus, the size distribution of spherical bubbles is expected to have a minor effect on the percelation threshold However, Burgisser et al. (2017) demonstrated with their experimental samples that the separation distance between bubbles weighted by the polydispersity of the bubble sizes affected the percolation

766 On the other hand, bubble shape plays a significant role in controlling the percolation threshold.

767 Garboczi et al. (1995) demonstrated that the continuum percolation threshold for randomly oriented, 768 inter-penetrating, prolate ellipsoids decreased from the porosity value for spheres of 0.29 (e.g., Lorenz 769 and Ziff 2001) to 0.26 for an aspect ratio of 2 , to 0.18 for an aspect ratio of 4 , to 0.09 for an aspect ratio 770 of 10 , to 0.007 for an aspect ratio of 100 , and to 0.0001 for an aspect ratio of 500 . Thus, the presence 771 of a small fraction of ellipsoidal bubbles (e.g., tube pumices) or cracks in a sample (e.g., tube pumices) 772 could provide an explanation of the permeabilities of volcanic foams whose porosities are below the 773 percolation threshold for mono-disperse spheres.

774 Almost all permeability determinations for porosities between 0.30 and 0.50 cluster between $10^{-14}$ and $77510^{-12} \mathrm{~m}^{2}$ (Fig. 8). Each individual study of permeability porosity relationships plotted in Figure 8 776 shows only modest increases (one to two orders-of-magnitude) in permeability between 0.30 and 0.50 777 porosity, and then only about an order-of-magnitude increase between 0.50 and the maximum poresity 
778

779

780

781

782

783

784

785

786

787

788

789

much less significantly than the first pathway.

790 For porosities between 0.5 and 0.9 , the permeabilities in Figure 8 range from values as low as $\sim 10^{-14}$

$791 \mathrm{~m}^{2}$ (Lindoo et al. 2016) to as high at $10^{-10} \mathrm{~m}^{2}$ (Bai et al. 2010). In general, silicic foams have lower

792 permeabilities and mafic melts higher permeabilities, but the dacitic foams with greater than 0.80

793 porosity measured in this study have permeabilities similar to basaltic foams with similar porosities

794 (Sarr and Manga 1999; Baiet al. 2010).

795

796

797

798 Because of our lack of knowledge of the critical porosity for each sample, the data were fit by

799

800

trachyandesitic foams in this porosity region display only modest increases; however, the basaltic

foams have an apparent factor of ten increase in the permeability between -0.50 and 0.55 porosity

(Table 2, Fig. 7). Note that these measurements were made on two different chips of basaltic melt;

which complicates the interpretation because the two samples have slightly different time-temperature

histories, and bubble popping was observed in the experiment with 0.50 porosity, both of which might

ereate small differences in foam porosity and permeability. Thus, we doubt that the factor of ten

increase in permeability between $\sim 0.50$ and 0.55 porosity for these basaltic samples is significant.

The slow increase in permeability at porosities above the percolation threshold at $\sim 0.3$ indicates that once a permeable pathway is created, the addition of other pathways for gas transport at higher porosities (as shown by higher valnes of $\beta$ and lower values of tortmosity) increases the permeability

\author{
(Seme
}

The peresity permeability data set contains many determinations at poresities below the nominat

eritical value for mono-disperse spheres. Each specific sample may have its own critical porosity based upon the size distribution and shape of the bubbles and pore throats in the sample, as discussed above.

99 empirical power laws without including a critical porosity. Although the data in Figure 8 can be empirically fit with a single power law, the dispersion of the permeabilities around this average fit is 1 
801

802 803 808 law equations.

809

810

orders of magnitude at porosities from 0.15 to 0.90 (Fig. 8). The range of permeabilities displayed in

Figure 8 also can be bound by two power law fits. The lower bound is constrained by the low porosity, low permeability measurements of Saar and Manga (1999) on basaltic foams, and rhyolitic porosities and permeabilities determined by Lindoo et al. (2016) and Burgisser et al. (2017): . The upper bound is dominated by the high permeability basaltic foams of Saar and Manga (1999) and of Bai et al. (2010), and can be described by a power law of. More than $92 \%$ of the viscous permeability determinations in Figure 8, including those of this study, fall within the envelope of these two power-

Although these simple power law relationships between porosity and permeability support $\underline{\text { Although }}$ percolation theory and the simple power-law relationships between porosity and permeability support the expected relationship between these two foam properties, permeability variations of up to 4 ordersof-magnitude at similar porosities clearly indicate that, in addition to porosity, there are other properties of the foams, such as the size distributions of bubbles and pore throats, influencing their permeability, as discussed above.

$A$ further control on permeability-poresity relations is the presence of crystals. $\underline{\text { Both crystallinity and }}$ bubble anisotropy have been shown to influence the permeability of natural and experimental $\underline{\text { magmatic foams, however these influences were not investigated in this study. Degruyter et al. (2010), }}$ $\underline{\text { Schneider et al. (2012), and Burgisser et al. (2017) provide multiple examples of the effects of }}$ preferred bubble orientation on magma permeability. The effect of crystals was not investigated in this study, but interested readers can find research-on permeability development in erystal-bearing magmatic foams was investigated experimentally in Bai et al. (2011) and Lindoo et al. (2017).

22 Nevertheless, the permeabilities of crystal-rich magmatic samples determined by Saar and Manga 23 (1999), Mueller et al (2008), Bai et al. (2011), Farquharson et al. (2015), Kushnir et al. (2016), and 
\$24 Lindoo et al. (2017) also plot in the same region as the aphyric-to-low-crystallinity samples, but are not

825 shown in Figure 8.

827 Comparison of measurements with the models of Degruyter et al. (2010) and Burgisser et al. (2017)

828 The complexity of the relationship between porosity and permeability displayed in Figure 8 has been

829 noted before by many authors, and many models for the calculation of permeability have been

830 constructed and demonstrated to reproduce the results of the individual studies (e.g., Saar and Manga

831 1999; Mueller et al., 2005; Polacci et al., 2008; Bai et al., 2010; Degruyter et al., 2010; Lindoo et al.,

832 2016; Burgisser et al. 2017; LaSpina et al. 2017). Most recently, Burgisser et al. (2017) developed a

833 model for permeability calculations based on modifications and extensions of concepts and equations

834 developed in Degruyter et al. (2010) and successfully applied their new model to multiple data sets.

835 Burgisser et al. (2017) used a modification of the channel-based Carman-Kozeny relationship to model

836 viscous permeability:

$$
k_{1}=\frac{\Phi_{c}^{n} d_{t}^{2}}{16 \times \tau^{2}},
$$

839 where $\phi_{c}^{n}$ is the connected porosity raised to the nth power, $d_{t}$ is the characteristic diameter of pore

840 throats, $\chi$ is the channel circularity:

$$
\chi=\left(\frac{r^{2}}{l^{2}}+\frac{l^{2}}{r^{2}}\right),
$$

842 where $r$ is the equivalent circle radius of the throat and 1 is its major axis $(\chi=2$ for circular pore

843 throats, Degruyter et al. 2010), and $\tau$ is the tortuosity. Degruyter et al. (2010) set $\mathrm{n}=1$ in this equation 844 for their study, but Burgisser et al. (2017) fit their data with this equation and determined a value of $n=$ 
845 2.49. Burgisser et al. (2017) found that this fit reproduced 26 of their 28 viscous permeability

846 measurements to within one log unit.

847 Equation $\underline{6} 3$ was applied to the foamsamples produced in this study for which the appropriate variables

848 were measured (Table 2) to predict permeabilities using both the value of $n=1$ from Degruyter et al.

849 (2010) and of $n=2.49$ from Burgisser et al. (2017); in both applications the value of $\chi$ was set to 2

850 (Degruyter et al. 2010). The quality of the model fit to the data was assessed by calculating chi-

851 squared as defined by:

$$
\text { chi }- \text { squared }=\sum \frac{(\log [\text { calculated permeability }]-\log [\text { measured permeability }])^{2}}{((\log [\text { measured permeability }]))} .(\underline{8} 5)
$$

853 Application of the Degruyter et al. (2010) formulation of Equation $\underline{6} z$ with $d_{t}$ equalingequalling the 854 average pore-throat diameter, $\chi=2$ and $n=1$ predicted the permeabilities of 17 out of 23 permeability 855 determinations to within $1 \log$ unit (all were within $1.6 \log$ units) and produced a chi-squared value of 8561.13 (Fig. 9a). With Burgisser et al.'s (2017) value of $n=2.49,19$ permeability predictions were within $8571 \log$ unit of our determinations (all were within $1.4 \log$ units), and the chi-squared value was 1.14 (Fig. 858 9a). The fit of these models to the measurements of this study is impressive when considering the 859 almost 5 orders of magnitude spread in the lattice-Boltzmann measured-permeabilities; however the 860 trend between predicted and measured permeabilities is at high angles to the slope of the perfect 1:1

861 correlation line between model and measurement (Fig. 9a), indicating the need for further model 862 improverefinement.

864 Toward a better model of the viscous permeability of magmatic foams by considering the largest pore 865 throat area

866 We wish to improve upon the models of Degruyter et al. (2010) and Burgisser et al. (2017) using the 
lattice-Boltzmann permeabilities found in this study. However, there are three caveats that must be

868

considered for the model discussed below. The first is that the model is only calibrated for

permeabilities between $\sim 10^{-15}$ and $10^{-10} \mathrm{~m}^{2}$. The second is that we have no technique to determine

when an individual sample becomes permeable, although we can approximate the permeability

$\underline{\text { threshold at } \Phi \sim 0.3 \text { (the percolation threshold for interpenetrating spheres) or use the methods }}$

presented in Burgisser et al. (2017) to estimate the permeability threshold if no other information is

available. The third caveat is that the model is probably only applicable to isotropic, or nearly

isotropic, samples.

Fluid conductivity in porous media can be related to a random resistor network by application of percolation theory (Stauffer and Aharony 1994; Blower et al. 2001). Applying this paradigm, the porous network in foams can be envisioned as resistors interconnected to one another that $\theta$-create a continuous circuit that when the permeability threshold is exceeded and allows fluid cante flow across the sample. The connections between resistors in the network can be either in parallel or in series (Stauffer and Aharony 1994; Blower et al. 2001). The conductivity of the network is related to the specifics of the pore throat cross-sectional area, as expressed in the numerator of the Carmaen-Kozeny relationship as the square of the characteristic throat diameter, $d_{c}{ }^{2}$ (Equation $\left.\underline{6}\right\}$ ). Given a complete description of the lengths and diameters of the pore throats, together with detailed information about their connections in either series or parallel sub-circuits, a complete model of sample permeability should be calculable, but these data are not available. Such a calculation would be tedious and we do not have all of the information required to solve the necessary equations. However, by making the simplifying assumption that the largest pore throat dominates the permeability (as the minimum-valued resistor in a parallel circuit dominates the current flow), the width of that throat, $\mathrm{d}_{\max }$, can be used as the characteristic pore throat diameter in Equation 3․․ Support for the idea of parallel connections 
891 (Table 2). Replacing the average throat diameter with the maximum throat diameter, and leaving $\chi=2$, 892 in Equation $\underline{6} 3$ did not significantly improve the fit of either the Degruyter et al. (2010) or Burgisser et 893 al. (2017) models; however the Burgisser et al. (2017) model yielded calculated permeabilities

894 consistently above the measured ones by a factor of 5. Based upon this observation, an empirical value 895 of $\chi=10$ (or a fitting factor of 5 times the original value of $\chi=2$ ) was chosen, and the resulting fit of 896 the Burgisser et al. model to the data is remarkable (Fig. 9b), with all but one of the measurements 897 reproduced to within one log unit and a chi-squared value of 0.01. The Degruyter et al. (2010) model 898 also reproduces all but one of the measurements to within $1 \log$ unit, but yields a chi-squared value 899 twice that of the Burgisser et al. model, 0.02.

900 The challenge in applying this model to predict permeability from porosity measurements is that the 901 tortuosity and the maximum throat diameter needed for the calculation typically are not known (cf.

902 Burgisser et al. 2017). In most cases, published studies only provide the average bubble diameter and 903 the porosity. However, the relationship found in this study between tortuosity and porosity (Eqn. $\underline{5} z \mathrm{a})$ 904 can be used to estimate the tortuosity. Additionally, we found that the maximum throat diameter can 905 be related to the average bubble size, $\mathrm{d}_{\mathrm{avg}}{ }^{\text {bubble }}(\mathrm{m})$, by

$$
d_{\max }=2.22251 \times 10^{-5} \ln \left(d_{\mathrm{avg}}^{\text {buble }}\right)+2.69501 \times 10^{-4}
$$

908 To test this model, the permeabilities of samples within the range of our calibration, $10^{-15}$ to $10^{-10} \mathrm{~m}^{2}$, 909 from Bai et al. (2010) and the isotropic pumices of Burgisser et al. (2017) were estimated using the 910 correlations between tortuosity and porosity and between the average bubble size and the maximum 911 throat diameter (Fig. 9c). We did not apply the model to the non-isotropic samples of Burgisser et al. 912 (2017) because we are unsure whether our correlations would apply to these samples and lack the 
913 necessary data to test our model on non-isotropic samples.

914 Although there is clearly a degradation in the accuracy of the model when the correlations are used to

915 estimate the tortuosity and maximum throat diameter rather than their measured values (cf. Fig. 9c with

916 Fig. 9b), the permeability of permeability of 11 of the 14 samples from Bai et al. (2010) are

917 reproduced within $1 \log$ unit, and the maximum difference between theall estimated and measured

918 permeabilities is $1.9 \log$ units. The chi-squared value (Eqn. 8) for all of the Bai et al. (2010) and the

919 model is 0.77 . The - The estimated permeabilities of all 13 isotropic samples from Burgisser et al.

920 (2017) are within 0.9 of a $\log$ unit of the measured values, and the chi-squared value is 0.35 . The

921 accuracy of this model is similar to that reported by Burgisser et al. (2017) who found that their fit to

922 Equation 6 could reproduce 26 out of the 28 (isotropic and anisotropic) samples they investigated to

923 within $1 \log$ unit.

924 This test of the model indicates its utility for estimating the permeability of samples with knowledge of 925 only the porosity and the average bubble diameter. However, as shown in Figure 9c, the model has a 926 tendency to overestimate the permeabilities by a factor of $\sim 5$. An ad hoc correction could be made for 927 this overestimation, but even without such correction the test indicates that permeabilities can be 928 calculated to within an order of magnitude with the model.

929 However, there are three caveats that must be considered. The first is that the model is only calibrated 930 for permeabilities between $-10^{-15}$ and $10^{-10} \mathrm{~m}^{z}$. The second is that we have no technique to determine 931 when an individual sample becomes permeable, although we can approximate the permeability 932 threshold at $\Phi-0.3$ or use the methods presented in Burgisser et al. (2017) to estimate the permeability 933 threshold if no other information is available. The third is that the model is probably only applicable to 934 isotropic, or nearly isotropic, samples. 
937 Although we were unable to successfully determine permeabilities at the same porosity from 938 experiments with the same composition at different heating rates, our results agree-we concur with 939 previous studies indicating that bubble growth rates significantly influence permeabilities (Rust and Cashman 2004; Burgisser and Gardner 2004; Mueller al. 2005, 2008; Takeuchi et al. 2009; Castro et al.

941 2012; Lindoo et al. 2016). In particular, Lindoo et al. (2016) noted that increasing decompression rates

942 in their experiments, leading to increasing bubble growth rates, resulted in an increasing percolation

943 threshold. This hypothesis is consistent with two observations on the basaltic composition where the

944 slowly heated experiment DRB2012-7f-10 $\left(1^{\circ} \mathrm{C} \mathrm{s}^{-1}\right)$ with a porosity of 0.55 has a permeability $(1.5 \mathrm{x}$

$\left.94510^{-11} \mathrm{~m}^{2}\right)$ similar to the rapidly heated $\left(6^{\circ} \mathrm{C} \mathrm{s}^{-1}\right), 0.73$ porosity experiment DRB2012-7c-f $\left(2.9 \times 10^{-11}\right.$

$946 \mathrm{~m}^{2}$ ), whereas the fit from Bai et al. (2010) predicts a permeability of at least $5 \times 10^{-11} \mathrm{~m}^{2}$ at a porosity 947 of 0.73 .

948 We also note that there may be a correlation between the bubble growth rate and the size distribution of 949 pore throats that significantly influences permeability. We propose this tentative hypothesis because of 950 the often-lower permeabilities of the rapidly heated $\left(5^{\circ} \mathrm{C} \mathrm{min}^{-1}\right)$ andesitic foam in comparison to the 951 more slowly heated $\left(\sim 1^{\circ} \mathrm{C} \mathrm{min}^{-1}\right)$ trachyandesitic and dacitic foams with similar porosities (Table 2, 952 Figure 7).

953 Despite the need for further experiments to quantitatively constrain the effects of decompression and 954 growth rates on the permeability of silicate foams, we suggest that the orders-of-magnitude variability 955 seen in permeability at similar porosities in Figure 8 is significantly controlled by the bubble growth 956 rate. The formation of a pore throat between two bubbles requires them to partially coalesce; for 957 coalescence to occur the interbubble melt film (IBF) must thin to the point where it fails, estimated to 958 be a thickness of $0.5 \mu \mathrm{m}$ in a rhyolitic melt by Castro et al. (2012). The rate at which the IBF thins is a 
959 function of the surface tension and bubble size (Equation 4), and the timescales of thinning vary from

960 less than a second (basaltic melts) to thousands of seconds (dacitic melts), based upon the analyses of

961 Castro et al. (2012) and Nguyen et al. (2013). In the case where bubbles are growing on a timescale

962 shorter than that of IBF thinning, coalescence is not as effective in creating large pore throats as at

963 slower growth rates, and permeabilities at equal porosities are lower than in the case where bubble

964 growth is slower than IBF thinning. Consideration of the lubrication and drag forces during bubble

965 growth suggests the possibility of a bubble-size-dependence of connectivity (and therefore

966 permeability). These considerations will be explored in future studies.

967 Although we think that the rate of gas exsolution plays a significant role in creating the wide spread of

968 permeability at equivalent porosities, we recognize that other factors may affect the observed variations

969 in permeability, such as the effects of bubble orientation (e.g., Degruyter et al. 2010; Burgisser et al.

970 2017) and the presence of crystals in the magma (e.g.ef., Bai et al. 2011; Lindoo et al. 20167).

972 A brief discussion of inertial permeability

973 Although the inertial permeability, $\mathrm{k}_{2}$, was not investigated in this study, earlier studies have correlated

974 the variation of $k_{2}$-with the viscous permeability, $k_{1}$. Note that such relationships exist because $k_{1}$ and

$975 \mathrm{k}_{2}$ are defined so that they are independent of the fluid (viscosity and density) and its flow regime

976 (Reynolds number), i.e., $k_{4}$ and $k_{z}$-depend only on pore size and shape. Rust and Cashman (2004)

977 found that for silicic foams

978

- , (7)

whereas the measurements on pumice by Yokoyama and Takeuchi (2009) yielded 
981 Bai et al. (2010) provided the following relationship for basaltic foams:

982

983 and Polacci et al. (2014) combined the available data and found

984

$(10)$

985 Most recently, Burgisser et al. (2017) found a relationship almost identical to that of Rust and Cashman 986 (2004):

987

988 Burgisser et al. (2017) also demonstrated the similarity of their relation to previous measurements of 989 visceus and inertial permeabilities.

990 Near $\mathrm{k}_{1}=10^{-12} \mathrm{~m}^{2}$, these relations (Equations 711 ) converge with a $\mathrm{k}_{2}$ range of 1.5 orders of 991 magnitude between the maximum of $7 \times 10^{-8} \mathrm{~m}$ (Bai et al. 2010) and minimum of $2 \times 10^{-9} \mathrm{~m}$

992 (Yokoyama and Takeuchi 2009). The calculated values of $k_{z}$-diverge at higher viscous permeabilities

993 until a value of $\mathrm{k}_{1}=10^{-10} \mathrm{~m}^{2}$ at which the inertial viscosities vary by only by about $\sim 2.5$ orders of 994 magnitude, from 4 × $10^{-7} \mathrm{~m}$ (Yokoyama and Takeuchi 2009) to 1 × $10^{-4} \mathrm{~m}$ (Rust and Cashman 2004).

995 Application of Equation 11 allows the viscous permeabilities, such as those determined in this study, to

996 be used to estimate the inertial permeabilities that are often needed for modeling gas flow in volcanic

997 systems (e.g., Rust and Cashman 2004; Burgisser et al. 2017). However, a single relationship between

$998 k_{4}$ and $k_{2}$ is unlikely to be applicable for all samples and without direct measurements of inertial

999 permeabilities in samples under investigation the estimates may be inaccurate.

1001 The competion between magmatic ascent and outgassing in magmatic systems

1002 The experimental results demonstrate significant differences in the size distributions of bubbles and 
1003 pere threats in the studied compositions. These morphological variations result in permeabilities that

1004 ean vary by at least an order of magnitude as seen in the results of this study and by even greater values

1005 when previous studies are included (Fig. 8). The various permeability porosity relationships shown in

1006 Figure 8 are expected to influence the ascent and eruption of magmas because of the role of gas in

1007 voleanic processes. To evaluate the significance of the differing permeability porosity relationships on

1008 magma ascent we applied the model of Namiki and Manga (2008).

1009 Although subvoleanic magma ascent and voleanic eruptions are driven by rapid volatile exsolution and

1010 formation of magmatic foams, expansion of volcanic foams increases their permeabilities, enhancing

1011 gas transport out of the magma and may slow, or possibly even stop, eruptions. This competition

1012 between the rapid expansion of magmatic foams and their loss of gas affects the intensity of volcanic

1013 eruptions. Combining the perosity permeability relationships in Figure \& with the steady state model

1014 for voleanic eruptions of Namiki and Manga (2008), we investigated the conditions at which porous

1015 flow removes gas from basaltic and rhyolitic magmatic systems at rates greater than the transport of the

1016 magma caused by volatile exsolution and bubble formation.

1017 Namiki and Manga (2008) constructed their model using a constant diameter magmatic conduit in

1018 which a water bearing magma ascends at velocity $v_{\theta}$ until the magma begins to vesiculate, causing the

1019 velocity to become a function of depth, $v(\mathrm{z})$. The ratio $v(\mathrm{z}) / \mathrm{v}_{\theta}$ is calculated from mass conservation:

1021 The porosity as a function of depth, is calculated by subtracting the water-saturation concentration at 1022 each pressure from the initial water concentration of the melt ( $2 \%$ for basalt, $4 \%$ for rhyolite in this 1023 study) to yield the exsolved water concentration, $C_{e}$. Water solubility in basalt was calculated from the 1024 results of Dixon et al. (2005) up to $70 \mathrm{MPa}$ with the addition of a solubility value of 0.1 wt\% $\mathrm{H}_{2} \mathrm{O}$ for 1025 water saturation at 1 bar; water solubility for the rhyolitic system was calculated from the data of Silver 
1026 et al. (1990) and Liu et al. (2008) for pressures to $180 \mathrm{MPa}$. These values were combined with the

1027 density of the melt, $\rho_{\mathrm{m}}\left(2800 \mathrm{~kg} \mathrm{~m}^{-3}\right.$ for basalt and $2400 \mathrm{~kg} \mathrm{~m}^{-3}$ for rhyolite $)$, and the density of water, $1028 \rho_{w}$, to calculate :

1030 where $\rho_{w}$ was calculated assuming ideal gas behavior for water at 1200 and $850^{\circ} \mathrm{C}$ for the basaltic and 1031 rhyolitic systems, respectively. Pressure was converted to depth using:

1033 with $g=9.8 \mathrm{~m} \mathrm{~s}^{2}$. Namiki and Manga (2008) expand the depressurization rate of a parcel of magma as

1035 and they calculate permeable gas flow by (from Ingebritsen and Sanford 1998):

1037 where $\beta$ is the gas compressibility (= $1 / \mathrm{P}$ for an ideal gas), $\mathrm{k}$ is the permeability, and $\eta_{\mathrm{g}}$ is the gas

1038 viscosity $\left(10^{-5} \mathrm{~Pa} \mathrm{~s}\right)$. Combining Equations 15 and 14 produces the criterion that Namiki and Manga 1039 (2008) propose as a measure of efficient outgassing:

1041 To solve this equation, the second derivative of pressure with respect to depth can be replaced by

1043 Tocreate an illustrative model, we considered an initial magma ascent velocity of $0.001 \mathrm{~m} \mathrm{~s}^{-}$, or about

$10440.1 \mathrm{~km} \mathrm{day}^{-}$. This ascent rate is equivalent to the ascent rate of the andesitic experiment's estimated

1045 equivalent decompression rate (discussed in the Methods section) calculated for a constant density of

$10462400 \mathrm{~kg} \mathrm{~m}^{-3}$ and to the effusive ascent rates of natural magmas tabulated by Browne and Szramek 
1047 (2015). The porosity-permeability relationships of both the basaltic magma and rhyolitic magma were modeled with the three fits (upper bound, average, and lower bound) to the porosity-permeability data 1049 in Figure 8.

1050 The results of the different cases considered are plotted in Figure 10, which displays the ratio of the gas 1051 velocity to the melt velocity as a function of depth for the basaltic and rhyolitic magma systems. The 1052 results of the low permeability cases are unsurprising. The gas velocities in neither the basaltic magma 1053 nor the rhyolitic magma approach those of the melt, and therefore gas is not expected to be lost during 1054 ascent (Fig. 10). Although the average permeability fit displays a maximum $\forall_{\text {gas }} / \forall_{\text {melt }}$ that is orders of 1055 magnitude higher than the low permeability fit, the calculations do not reach $v_{\text {gas }} / v_{\text {melt }}=1$ (Fig. 10). 1056 The observation that the Namiki and Manga model predicts that foams whose permeability is defined 1057 by the average fit should not lose the gas driving their transport is consistent with the eruption of the 1058 many natural, vesicular samples for which porosity permeability measurements constrain the average 1059 fit in Figure 8. With no loss of the vesiculating gas, the modeled magmas with either low or average 1060 porosity permeability relations reach velocities similar to the explosive ascent rates of natural magmas 1061 tabulated by Browne and Szramek (2015) of 0.05 to $3 \mathrm{~m} \mathrm{~s}^{-1}$ -

1062 The high permeability cases for both the basaltic and rhyolitic magmas display regions where the gas 1063 velocity exceeds that of the melt and has the potential to escape (Fig. 10), lessening or removing the 1064 driving force for volcanic eruptions. What is most surprising is that both melt compositions cross the 1065 eritical $\vee_{\text {gas }} / v_{\text {melt }}$ ratio of 1 and into the region of potential gas loss at low vesicularities, 0.07 and 0.12 1066 for the basaltic and rhyolitic magmas, respectively, and maintain velocity ratios above 1 until 1067 vesicularities reach $\sim 0.87$ (Fig. 10). This modeling suggests that even low porosity magmas have the 1068 eapacity to lose their driving gases if they are sufficiently permeable. The paucity of natural samples 1069 with porosity permeability relationships similar to those of the high-permeability case is consistent 


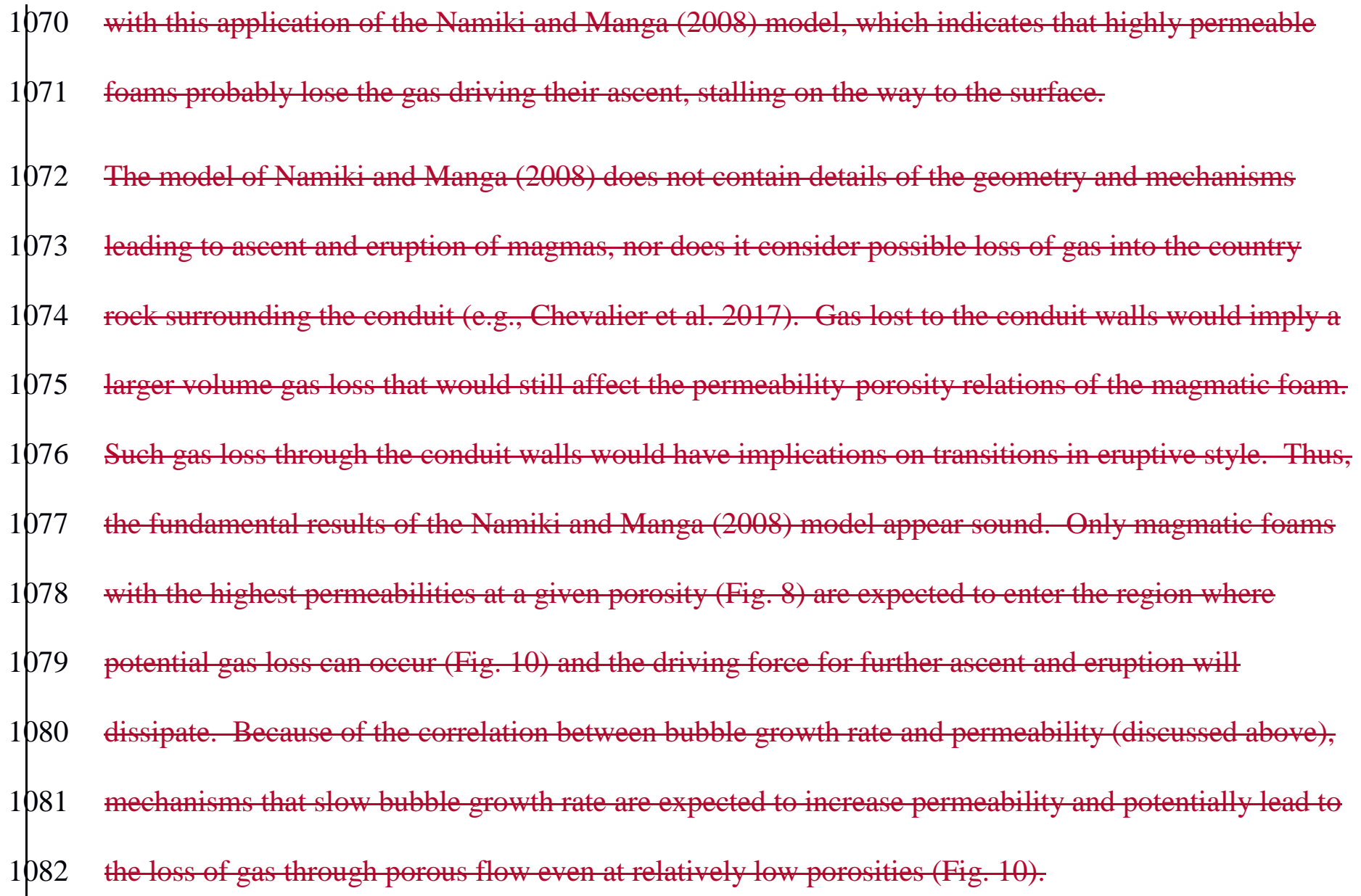

1085 A complete characterization of magmatic foams is required to model permeability because 1086 permeability-porosity relationships alone do not provide sufficient data for accurate modeling and 1087 prediction. Furthermore, average properties of the foam, in particular average pore-throat diameters, 1088 appear to be insufficient to fully characterize permeability. Complete measurements of porosity, 1089 bubble and pore throat size distributions, as well as tortuosity, are required to model accurately the 1090 permeability of magmatic foams. In particular, we stress the apparent importance of the largest pore 1091 throat on the permeability of magmatic foams. We propose one such model, which we consider a step 1092 in the right direction, that can be used to benchmark future studies. Combining $€$ The results of this 
1093 study are consistent with previous work indicatinges the importance of the bubble growth rate on the 1094 permeability of magmatic foams. Higher growth rates appear to produce lower permeabilities, and the 1095 effect of growth rate on permeability may explain a significant portion of the orders-of-magnitude 1096 spread in permeabilities at similar porosity. The model of Namiki and Manga (2008) is consistent with

1097 measurements of natural samples and predicts that only magmatic foams with the highest permeability 1098 values may lose the gases driving their ascent. Remarkably, this model demonstrates that gas loss can 1099 eccur at small porosities as well.

1101 Acknowledgements

1102 D.R. Baker thanks NSERC for their continued support for his research through the Discovery Grant 1103 Program. All of the members of the TOMCAT team and of the Swiss Light Source at the Paul Scherrer 1104 Institut are thanked for their creation of the facility that allowed this study to be done and their 1105 continuing dedication to providing support to external users of the beamline.

1106 We also thank the editor, KE. Cashman and the reviewers H. Wright, L. Chevalier and A. Burgisser for 1107 their detailed and thoughtful comments that significantly improved the presentation of our research in 1108 this contribution.

1110 References

1111 Al-Raoush RI, Madhoun IT (2017) TORT3D: A MATLAB code to compute geometric tortuosity from 1112 3D images of unconsolidated porous media, Powder Tech 320: 99-107

1113 Archie GE (1942) The electrical resistivity log as an aid in determining some reservoir characteristics. $1114 \quad$ Trans Am Inst Mineral Meteorol 146:54-62. 
1115 Bai L, Baker DR, Rivers M (2008) Experimental study of bubble growth in Stromboli basalt melts at 1 atm. Earth Planet Sei Lett 267:533-547

1117 Bai L, Baker DR, Hill RJ (2010) Permeability of vesicular Stromboli basaltic glass: Lattice Boltzmann 1118 simulations and laboratory measurements. J Geophys Res 115:B07201.

1119 doi:10.1029/2009JB007047

1120 Bai L, Baker DR, Polacci M, Hill RJ (2011) In-situ degassing study on crystal-bearing Stromboli

1121 basaltic magmas: Implications for Stromboli Eruptions. Geophys Res Lett 38: L17309

1122 Baker DR (2004) Piston-cylinder calibration at 400 to $500 \mathrm{MPa}$ a comparison of using water solubility 1123 in albite melt and $\mathrm{NaCl}$ melting. Am Mineral 89:1553-1556

1124 Baker DR Eggler DH (1987) Compositions of anhydrous and hydrous melts coexisting with plagioclase, 1125 augite, and olivine or low-Ca pyroxene from $1 \mathrm{~atm}$. to $8 \mathrm{kbar}$ : application to the Aleutian volcanic 1126 center of Atka. Am Mineral 72:12-28

1127 Baker DR, Polacci MR, LaRue A (2011) A study on the reproducibility of counting vesicles in volcanic $1128 \quad$ rocks. Geosphere, doi: 10.1130/GES00553.1

1129 Baker DR, Brun F, O’Shaughnessy C, Mancini L, Fife JL, Rivers M (2012) A four-dimensional X-ray 1130 tomographic microscopy study of bubble growth in basaltic foam. Nat Comm 3: 1135. 1131 doi:10.1038/ncomms2134

1133 Blower JD (2001) Factors controlling permeability-porosity relationships in magmas. Bull Volcanol 1134

1135 Bouvet de Maisonneuve C, Bachmann O, Burgisser A (2008) Characterization of juvenile pyroclasts from 1136 the Kos Plateau Tuff (Aegean Arc): insights into the eruptive dynamics of a large rhyolitic eruption. 
1138 Browne B, Szramek L (2015) Chapter 9: Rates of magma ascent and storage. The Encyclopedia of 1139 Volcanoes. Elsevier Inc. http://dx.doi.org/10.1016/B978-0-12-385938-9.00009-2

1140 Brun F, Dreossi D (2010) Efficient curve-skeleton computation for the analysis of biomedical 3D 1141 images. Biomed Sci Instrum 46:475-480

1142 Brun F, Mancini L, Kasae P, Favretto S, Dreossi D, Tromba G (2010) Pore3D: A software library for 1143 quantitative analysis of porous media. Nucl Instr Methods Phys Res A 615:326-332

1144 Burgisser A, Gardner JE (2004) Experimental constraints on degassing and permeability in volcanic 1145 conduit flow. Bull Volcanol 67:42-56. http://dx.doi.org/10.1007/s00445-004-0359-5

1146 Burgisser A, Chevalier L, Gardner JE, Castro JM (2017) The percolation threshold and permeability 1147 evolution of ascending magmas. Earth Planet Sci Lett 470:37-47

1148 Carman PC (1937) Fluid flow through a granular bed. Trans Inst Chem Eng London, 15:150-156

1149 Castro JM, Burgisser A, Schipper CI, Mancini S (2012) Mechanisms of bubble coalescence in silicic $1150 \quad$ magmas. Bull Volcanol 74:2339-2352

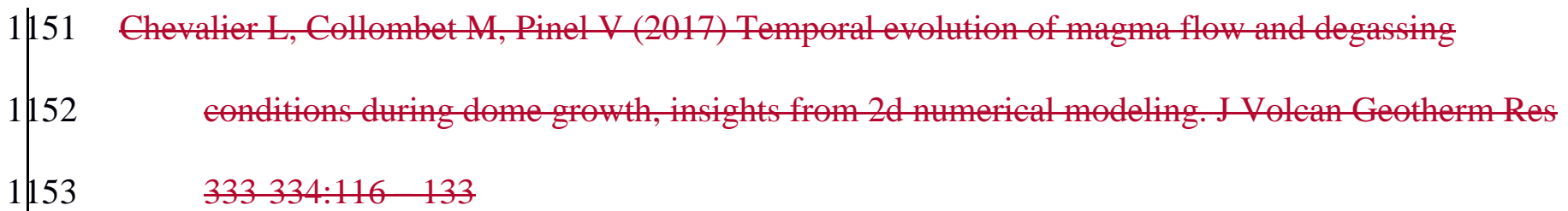

1154 Colombier M, Wadsworth FB, Gurioli L, Scheu B, Kueppers U, Di Muro A, Dingwell DB (2017) The 1155 evolution of pore connectivity in volcanic rocks. Earth Planet Sci Lett 462:99-109

1156 Consiglio R, Baker DR, Paul G, Stanley HE (2003) Continuum percolation thresholds for mixtures of 1157 spheres of different sizes. Physica A 319:49-55

1158 Cox SJ, Graner F (2004) Three-dimensionalThree-dimenstional bubble clusters: Shape, packing, and 
Đixon JE, Stolper EM, Holloway JR (2005) An experimental study of water and carbon dioxide solubilities in mid-ocean ridge basaltic liquids. Part I: Calibration and solubility models. J Petrol 36:1607-1631

1163 Degruyter W, Bachmann O, Burgisser A (2010) Controls on magma permeability in the volcanic

1164 1165

1166 1167 1168 1169 1170 1171 1172 1173 1174 1175 1176 1177 1178 Fife JL, Rappaz M, Pistone M, Celcer T, Mikuljan G, Stampanoni M (2012) Development of a laser1179 1180 conduit during the climatic phase of the Kos PlateauPleateau Tuff eruption (Aegean Arc). Bull Vulcanol 72:63-74

Domb C (1972) A note on the series expansion method for clustering problems. Biometrika 59:209-211

Dowd B, Campbell GH, Marr RB, Nagarkar V, Tipnis S, Axe L, Siddons DP (1999) Developments in synchrotron x-ray computed tomography at the National Synchrotron Light Source. Developments in X-Ray Tomography II, Proc SPIE 3772:224-236

Fagents SA, Gregg TKP, Lopes RMC (2013) Modeling Volcanic Processes: The Physics and Mathematics of Volcanism. Cambridge University Press, Cambridge, $421 \mathrm{p}$

Farquharson J, Heap MJ, Varley NR, Baud P, Reushclé T (2015) Permeability and porosity relationships of edifice-forming andesites: A combined field and laboratory study. Jour Volcan Geotherm Res 297:52-68

Ferguson DJ, Gonnermann HM, Ruprech P, Plank T, Hauri EH, Houghton BF, Swanson DA (2016) Magma decompression rates during explosive eruptions of Kilauea volcano, Hawaii, recorded by melt embayments. Bull Volcanol 78:71 based heating system for in-situ synchrotron-based x-ray tomographic microscopy, J. Synchrotron Radiation 19:352-358 
1181 Fortin M-A, Riddle J, Desjardins-Langlais Y, Baker DR (2015) The effect of water on the sulfur

1182 concentration at sulfide saturation (SCSS) in natural melts. Geochim Cosmochim Acta 160:100-

$1183 \quad 116$

1184 Gaonac'h H, Lovejoy S, Stix J, Scherzter D (1996) A scaling growth model for bubbles in basaltic lava 1185 flows. Earth Planet Sci Lett 139:395-409.

1186 Garboczi EJ, Snyder KA, Douglas JF, Thorpe MF (1995) Geometrical percolation threshold of 1187 overlapping ellipsoids. Physical review E 52:819-828

1188 Giordano D, Nichols ARL, Dingwell DB (2005) Glass transition temperatures of natural hydrous 1189 melts: a relationship with shear viscosity and implications for the welding process. J Volcan $1190 \quad$ Geotherm Res 142: 105-118

1191 Giordano D, Russell JK, Dingwell DB (2008) Viscosity of magmatic liquids: A model. Earth Planet $1192 \quad$ Sci Lett 278:123-134

1193 Hildebrand T, Rüegsegger P (1997) A new method for the model-independent assessment of thickness 1194 in three-dimensional images. J Microscopy 185:67-75

1195 Hill RJ, Koch DL,Ladd AJC (2001) The first effects of fluid inertia on flows in ordered and random 1196 arrays of spheres. J Fluid Mech 448:213-241. doi:Ziff10.1017/S0022112001005948

1197 Hill RJ, Koch DL (2002) The transition from steady to weakly turbulent flow in a close-packed ordered $1198 \quad$ array of spheres. J Fluid Mech 465:59-97. doi:10.1017/S0022112002008947

1199 Humphreys MCS, Menand T, Blundy JD, Klimm K (2008) Magma ascent rates in explosive eruptions: $1200 \quad$ Constraints from $\mathrm{H}_{2} \mathrm{O}$ diffusion in melt inclusions. Earth Planet Sci Lett 270:25-40

1201 Ingebritsen SE, Sanford WE (1998) Groundwater in Geologic Processes. Cambridge University Press, $1202 \quad$ Cambridge 
1203 Klug C, Cashman KV (1996) Permeability development in vesiculating magmas: implications for 1204 fragmentation. Bull Volcan 58:87-100

1206 Kraynik AM, Reinelt DA, van Swol F (2004) Structure of random foam. Phys Rev Lett 93: 208301, 1207 10.1103/PhysRevLett.93.208301

1208 Kushnir ARL, Martel C, Bourdier J-L, Heap MJ, Reushclé T, Erdmann S, Komorowski J-C, Cholik N 1209 (2016) Probing permeability and microstructure: Unravelling the role of a low-permeability 1210 dome on the explosivity of Merapi (Indonesia). Jour Volcan Geotherm Res 316:56-71

1211 LaRue A (2012) Bubble size distributions and magma-water interaction at Eyjafjallajökull volcano, 1212 Iceland. M.Sc. Thesis, McGill University

1213 La Spina G, Polacci M, Burton M, de’ Michieli Vitturi M (2017) Numerical investigation of 1214 permeability models for low viscosity magmas: Application to the 2007 Stromboli effusive 1215 eruption. Earth Planet Sci Lett 473:279-290. https://doi.org/10.1016/j.eps1.2017.06.013

1216 Lindoo A, Larsen JF, Cashman KV, Dunn AL, Neill OK (2016) An experimental study of permeability 1217 development as a function of crystal-free melt viscosity. Earth Planet Sci Lett 435:45-54

1218 Lindoo A, Larsen JF, Cashman KV, Oppenheimer J (2017) Crystal controls on permeability 1219 development and degassing in basaltic andesite magma. Geology 45: 831-834

1220 Lindquist WB (2002) Quantitative analysis of three dimensional X-ray tomographic images. Proc. $1221 \quad$ SPIE 4503:103-115

1222 Lindquist WB, Lee SM (1996) Medial axis analysis of void structure in three-dimensional tomographic 1223 images of porous media. J. Geophys Res 101:8297-8310

1224 Liu Y, Samaha N-T, Baker DR (2007) Sulfur concentration at sulfide saturation (SCSS) in magmatic 
1226 Liu Y, Zhang Y, Behrens $\mathrm{H}$ (2005) Solubility of $\mathrm{H}_{2} \mathrm{O}$ in rhyolitic melts at low pressures and a new 1227 empirical model for mixed $\mathrm{H}_{2} \mathrm{O}-\mathrm{CO}_{2}$ solubility in rhyolitic melts. J Volcan Geotherm Res $1228 \quad 143: 219-235$

1229 Lorenz CD, Ziff RM (2001) Precise determination of the critical percolation threshold for the three1230 dimensional 'Swiss cheese' model using a growth algorithm. J Chem Phys 114:3659-3661

1231 Marone F, Stampanoni M (2012) Regridding reconstruction algorithm for real time tomographic 1232 imaging. J Synchrotron Rad 19:1029-1037

1233 Mokso R, Marone F, Stampanoni M (2010) Real time tomography at the Swiss Light Source. AIP Conf $1234 \quad$ Proc $1234: 87-90$

1235 Mokso R, Schlepuetz CM, Theidel G, Billich H, Schmid E, Celcer T, Mikuljan G, Sala L, Marone F, 1236 Schlumpf N, Stampanoni M (2017) GigaFRoST: The Gigabit Fast Readout System for 1237 Tomography. Jour Synchrotron Rad doi.org/10.1107/S1600577517013522

1238 Mueller S, Melnik O, Spieler O, Scheu B, Dingwell DB (2005) Permeability and degassing of dome 1239 lavas undergoing rapid decompression: An experimental determination. Bull Volcan 67:526-538

1240 Mueller S, Scheu B, Spieler O, Dingwell DB (2008) Permeability control on magma fragmentation. $1241 \quad$ Geology 36:499-402

1242 Navon O, Lyakhovsky, V, (1998) Vesiculation processes in silicic magmas. In: Gilbert, J.S., Sparks, $1243 \quad$ R.S.J. (Eds.), The Physics of Explosive Volcanic Eruptions. Geol Soc Spec Pub Lond 145: 27$1244 \underline{50 .}$

1245 Namiki A, Manga, M (2008) Transition between fragmentation and permeable outgassing of low 1246 viscesity magmas. J Volcan Geotherm Res 169:48-60 
1248 Neuville DR, Courtial P, Dingwell DB, Richet P (1993) Thermodynamic and rheological properties of 1249 rhyolite and andesite melts. Contrib Mineral Petrol 113:572-581

1250 Newman MEJ (2005) Power laws, Pareto distributions and Zipt's law. Contemporary Physics 46:323$1251 \quad 351$

1252 Nguyen CT, Gonnermann HM, Chen Y, Huber C, Maiorano AA, Gouldstone A, Dufek J (2013) Film 1253 drainage and the lifetime of bubbles. Geochem Geophys Geosys 14: 10.1002/ggge.20198

1254 Ni H, Zhang L (2018) A general model of water diffusivity in calcalkaline silicate melts and glasses. $1255 \quad$ Chem Geol 478:60-68.

1256 Odgaard A, Gundersen HJG (1993) Quantification of connectivity in cancellous bone, with special 1257 emphasis on 3-D reconstructions. Bone 14:173-182

1258 Ogata R, Odagaki T, Okazaki K (2005) Effects of poly-dispersity on continuum percolation. J Phs 1259 Condens Matter 17:4531-4538

1260 Papale P, Moretti R, Barbato D (2006) The compositional dependence of the saturation surface of $\mathrm{H}_{2} \mathrm{O}$ $1261+\mathrm{CO}_{2}$ fluids in silicate melts. Chem Geol 229:78-95

1262 Polacci M, Baker DR, Bai L, Mancini L (2008) Large vesicles record pathways of degassing at basaltic 1263 volcanoes. Bull Volcanol 70:1023-1029. https://doi.org/10.1007/s00445-007-0184-8

1264 Polacci M, Baker DR, La Rue A, Mancini L, Allard P (2012) Degassing behaviour of vesiculated 1265 basaltic magmas: an example from Ambrym volcano, Vanuatu Arc. Jour Volcan Geotherm Res $1266 \quad 233-234: 55-64$.

1267 Polacci M, Bouvet de Maisonneuve C, Giordano D, Piochi M, Mancini L, Degruyter W, Bachmann O 1268 (2014) Permeability measurements of Campi Flegrei pyroclastic products: An example from the 

Campanian Ignimbrite and Monte Nuovo eruptions. Jour Volcan Geotherm Res 2272:16-22.

1270 Rivers ML, Wang Y (2006) Recent developments in microtomography at GeoSoilEnviroCARS. 1271 Developments in X-Ray Tomography V, Proc SPIE 6318:J3180. doi: 10.1117/12.681144

1272 Rust AC, Cashman KV (2004) Permeability of vesicular silicic magma: Inertial and hysteresis effects. $1273 \quad$ Earth Planet Sci Lett 228:93-107

1274 Rust AC, Cashman KV (2011) Permeability controls on expansion and size distributions of pyroclasts. $1275 \quad$ J Geophys Res 116:B11202

1276 Saar MO, Manga M (1999) Permeability-porosity relationship in vesicular basalts. Geophys Res Lett $1277 \quad 26: 111-114$

$\underline{\text { Schneider A, Rempel AW, Cashman KV. (2012) Conduit degassing and thermal controls on eruption }}$ styles at Mount St. Helens. Earth Planet Sci Lett 357-358: 347-354

1280 Shaw HR (1972) Viscosities of magmatic silicate liquids: an empirical method of prediction. Am J Sci 272:870-893

Silver LA, Ihinger PD, Stolper E (1990) The influence of bulk composition on the speciation of water in silicate glasses. Con Min Petrol 104:142 162

1284 Sparks RSJ (2003) Dynamics of magma degassing. In: Oppenheimer, C., Pyle, D.M., and Barclay, J. 1285 (eds) Volcanic Degassing. Geological Society, London, Special Publications 213:5-22

1286 Spieler O, Kennedy B, Kueppers U, Dingwell DB, Scheu B, Taddeucci J (2004) The fragmentation 1287 threshold of pyroclastic rocks. Earth Planet Sci Lett 226:139-148

1288 Stauffer D, Aharony A. (1994) Introduction to Percolation Theory, $2^{\text {nd }}$ Edition. Taylor \& Francis, $1289 \quad$ London, $181 \mathrm{p}$

1290 Takeuchi S, Nakashima S, Tomiya A (2008) Permeability measurements of natural and experimental 
$1291 \quad$ volcanic materials with a simple permeameter: Toward and understanding of magmatic degassing processes. J Volcan Geotherm Res 177:329-339.

1293 Takeuchi S, Tomiya A, Shinohara H (2009) Degassing conditions for permeable silicic magmas:

1294 implications from decompression experiments with constant rates. Earth Planet Sci Lett 283:1011295 110. http://dx.doi.org/10.1016/j.epsl.2009

1296 Thovert J.F., Salles J., Adler P.M. (1993) Computerized characterization of the geometry of real porous 1297 media: their discretization, analysis and interpretation. J. Microscopy 170:65-79

1298 Tomasi C, Manduchi R (1998) Bilateral filtering for gray and color images. Sixth Internat Conf Comp 1299 Vision: 839-846

1300 Wright HMN, Cashman KV, Gottesfeld EH, Roberts JJ (2009) Pore structure of volcanic clasts:

1301 Measurements of permeability and electrical conductivity. Earth Planet Sci Lett 280:93-104

1302 Yokoyama T, Takeuchi S (2009) Porosimetry of vesicular volcanic products by a water-expulsion 1303 method and the relationship of pore characteristics to permeability. J Geophys Res 114:B0221. 1304 doi:10.1029/2008JB005758

1305 Zandomeneghi D, Voltolini M, Mancini L, Brun F, Dreossi D, Polacci M (2010) Quantitative analysis 1306 of X-ray microtomography images of geomaterials: Application to volcanic rocks. Geosphere $1307 \quad 6: 793-804$

$1 \beta 08$ Zhou J-Q, Chen Y-F, Wang L, Rayani Cardenas M. (2019) Universal relationship between viscous and inertial permeability of geologica porous media. Geophys Res Lett 46:1441-1448.

1310 Ziff RM, Torquato S (2017) Percolation of disorderd jammed sphere packings. J Phys A Math Theor 1311 50: 085001, doi:10.1088/1751-8121/aa5664

1312 


\section{Figure Captions}

1314 Figure 1 a) Slice of complete sample DRB2012_6e_10. The $256 \times 256$ pixel region in this slice 1315 sampled for analysis is shown by the dashed lines. b) Detailed image of the portion of the $256 \times$ 1316256 slice sampled from the larger image. c) Thresholded image of (b) with a smaller $160 \times 160$ 1317 pixel region used for quantitative measurements shown enclosed by the solid lines. d) $1 \$ 18$ Reconstruction for DRB2012-2a-19, with the one-voxel-wide-skeleton in yellow and the nodes 1319 (intersections of the skeleton branches) in red and orange, demonstrating the effect of only

\section{Figure 2} $\mathrm{a}, \mathrm{b}, \mathrm{c})$ Selected $\underline{3 \mathrm{D}}$ renderings of andesitic experiment DRB2012-2a during vesiculation. In the earliest image the sample is approximately $1 \times 1 \times 2 \mathrm{~mm}$ in size. Due to the 


\begin{tabular}{|c|c|}
\hline 336 & perspective projections of these renderings the scale bars are only approximate. Representative \\
\hline 337 & interior sections of these samples were chosen for quantitative analysis. $\underline{\mathrm{d}, \mathrm{e}, \mathrm{fb}}$ ) Corresponding \\
\hline 38 & thresholdedThresholded tomographic slices (axial slice number 128 near the center of the \\
\hline 39 & tomegraphic reconstruction) from each of the renderings shown in-part panels $a_{2} b$ and $c$, in \\
\hline 340 & which black is the melt and white is either the bubbles in the samples or the air around samples \\
\hline $1 \beta 41$ & (seen in panels e and fthe early images only). The $500 \mu \mathrm{m}$ scale bar in panel d also applies to \\
\hline 1342 & panels e and f. - Melt viscosities are calculated using Giordano et al. (2008) with 5 wt. $\% \mathrm{H}_{2} \mathrm{O}$ in \\
\hline 1343 & the melt at $900{ }^{\circ} \mathrm{C}$ and an estimated $1 \mathrm{wt} . \% \mathrm{H}_{2} \mathrm{O}$ at $994^{\circ} \mathrm{C}$; at $1089{ }^{\circ} \mathrm{C}$, near the end of bubble \\
\hline 134 & growth, the melt is assumed to be anhydrous for the viscosity calculation. The numbers in each \\
\hline $1 \beta 45$ & panel refer to the time in seconds after the start of data acquisition whose qQuantitative \\
\hline 1346 & measurements of the experiment are provided in Table 2 and Supplemental Data Table 1. Please \\
\hline & data for a movie of this sample during bubble growth. \\
\hline
\end{tabular}

1348 Figure $3 \quad$ a) Bubble size distributions in basaltic sample DRB2012-7. The figure contains 1349 subpanels with bubble size distributions from different experiments with porosities from 0.50 to 1350 0.73. All panels in this and subsequent plots of bubble and pore-throat size distributions are $1351 \quad$ plotted in order of increasing experimental duration and temperature. Note that in this figure the sample with a porosity 0.50 was made by reheating the sample with 0.52 porosity (please see Table 2 and the text for further discussion). In each subpanel a volume-normalized histogram of the sizes of the bubbles (the bar graphs) is presented together with the cumulative distribution of the bubble sizes (solid black line). The porosity is given in the upper right corner of each subpanel. The mean bubble diameter, $\mathrm{d}$, and one-standard deviation about the mean is given inen the left side of each subpanel. Bin sizes are $5 \mu \mathrm{m}$. b) Corresponding pore throat size distributions in basaltic sample DRB2012-7. The information on the pore-throat distributions is 
presented in the same manner as the bubble size distributions in Part a. Bin sizes are $5 \mu \mathrm{m}$.

1360 Figure 4 Bubble and pore-throat distributions for andesitic experiment DRB2012-2a. a) Bubble 1361 size distributions for porosities between 0.17 and 0.33 . Bin sizes are $5 \mu \mathrm{m}$. b) Bubble size 1362 distributions for porosities between 0.41 and 0.59 . Bin sizes are $5 \mu \mathrm{m}$. c) Bubble size 1363 distributions for porosities between 0.71 and 0.80 . Bin sizes are $5 \mu \mathrm{m}$. d) Pore throat size 1364 distributions for porosities between 0.17 and 0.33 . Bin sizes are $5 \mu \mathrm{m}$. e) Pore throat size 1365 distributions for porosities between 0.41 and 0.59 . Bin sizes are $5 \mu \mathrm{m}$. f) Pore throat size 1366 distributions for porosities between 0.71 and 0.80 . Bin sizes are $5 \mu \mathrm{m}$. Please see caption to 1367 Figure 3 for a complete description of figure.

1368 Figure 5 Bubble and pore throat distributions for trachyandesitic experiment EFJ-8a. a) Bubble 1369 size distributions for porosities between 0.09 and 0.64 . Bin sizes are $5 \mu \mathrm{m}$. b) Corresponding 1370 pore throat distributions. Bin sizes are $5 \mu \mathrm{m}$. Please see caption to Figure 3 for a complete 1371 description of figure.

1372 Figure 6 Bubble and pore throat distributions for dacitic experiment DRB2012-6e. a) Bubble size 1373 distributions for porosities between 0.38 and 0.84 . Bin sizes are $5 \mu \mathrm{m}$. b) Corresponding pore 1374 throat distributions. Bin sizes are $5 \mu \mathrm{m}$. Please see caption to Figure 3 for a complete description 1375 of figure.

1376 Figure $7 \quad$ Experimental foam permeabilities determined by lattice-Boltzmann simulations plotted 1377 as a function of porosity. Uncertainties in the measured porosity and in the permeability are 1378 estimated at 20 relative percent and typically the same size as, or smaller than, the symbols. The solid blue line is the power-law fit to the lattice-Boltzmann permeabilities of the andesitic composition. The proposed relationships between porosity and permeability for basaltic and for silicic compositions from Bai et al. (2010) are also plotted. (Color on lineenline) Please see text 


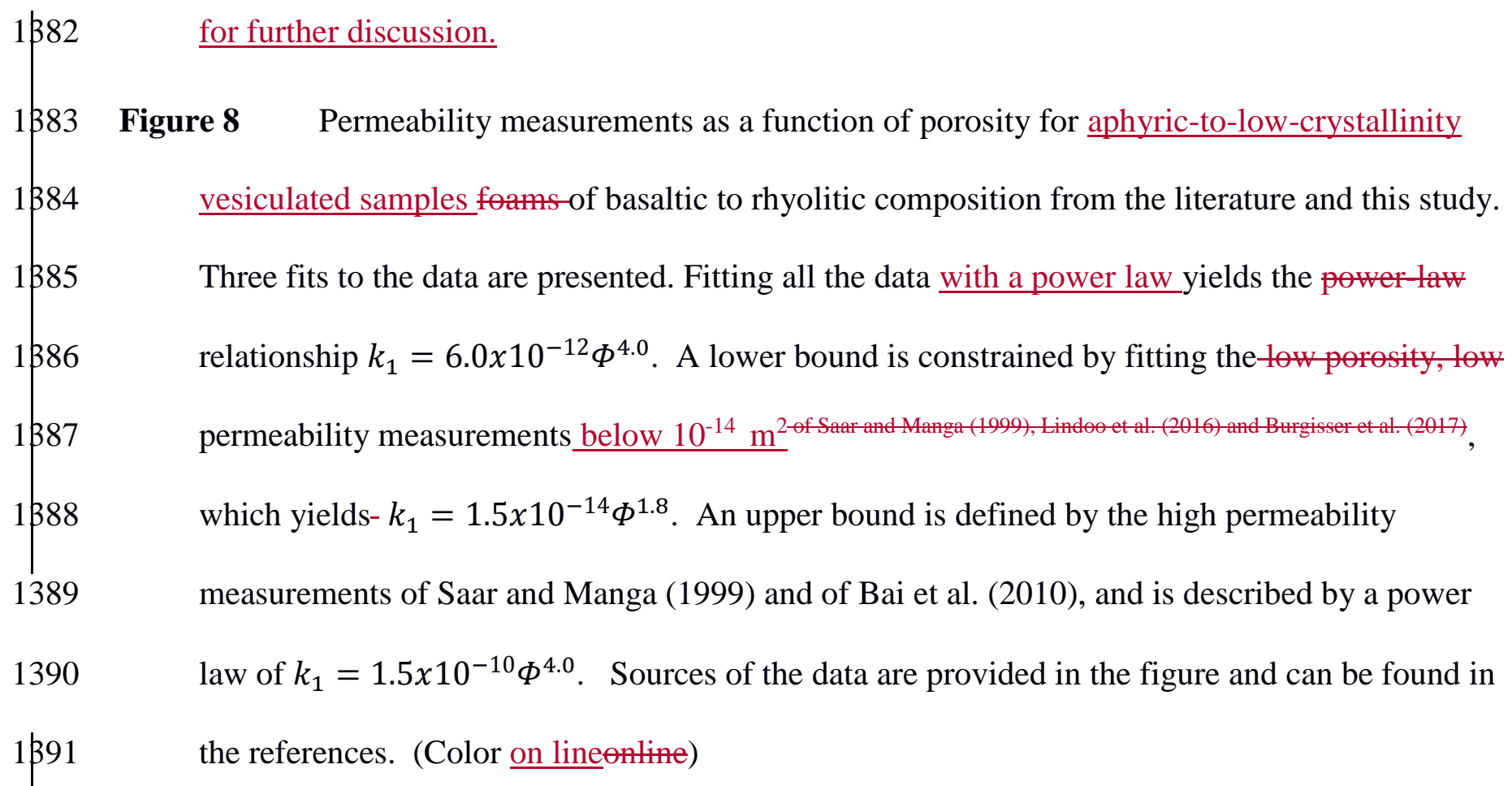

1392 Figure $9 \quad$ a) Application of the models for the prediction of permeability using the Carman1393 Kozeny equations of Degruyter et al. (2010) and of Burgisser et al. (2017). The line labeelled 1:1 represents a perfect fit of athe model to the data; the line labeled " $5 x$ " represents the modeled values multiplied by 5 and the line labeled " $0.2 x$ " represents the modeled values multiplied by 0.2. In these models the average pore throat value (Table 2) was used as the characteristic diameter of pore throats. b) Modification of the models of Degruyter et al. and of Burgisser et al. by using the maximum pore throat as the characteristic diameter and an empirical value of $\chi=10$. Note the excellent fit of the Burgisser et al. model to the measurements. c) Comparison of the modified Burgisser et al. model and measured permeabilities of samples from Bai et al. (2010) and of the isotropic samples from Burgisser et al. (2017) using relationships between the average bubble diameter and the largest throat diameter and between the porosity and the tortuosity, as determined in this study. The data from Bai et al. (2010) and from Burgisser et al. (2017) were not used to calibrate the model. Please see text for further discussion. (Color on lineenline) 


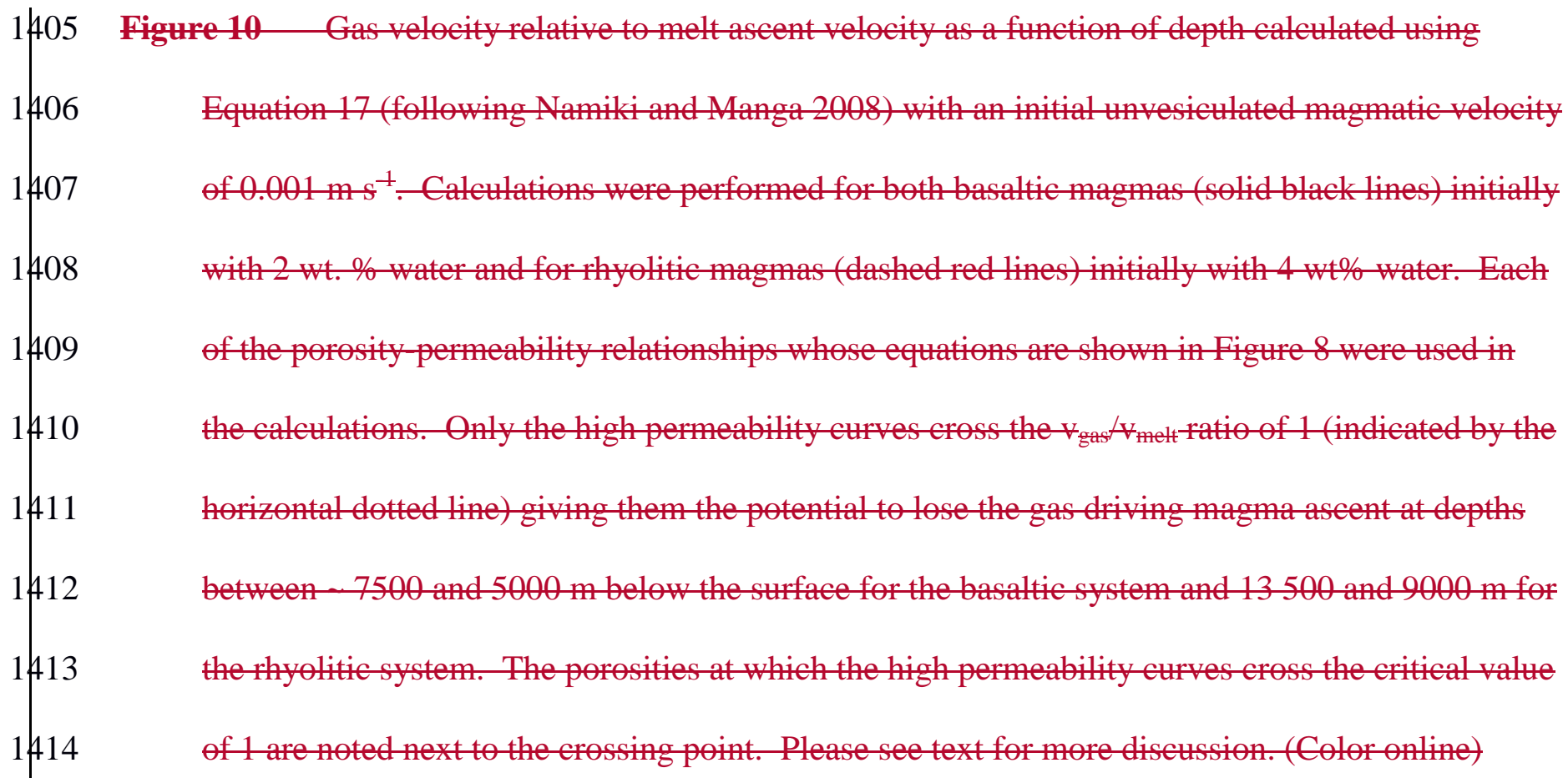

1416 Table $1 \quad$ Starting glass compositions

1417 Table $2 \quad$ Experimental results

1418 Supplementary Movie Bubble growth in DRB2012-2a

1419 Supplementary Data Table ___ Measured diameters of bubbles and pore throats in the 1420 experiments.

1421 Supplementary Figure 1 Connectivity versus porosity in the experimental samples (in color 1422 online). 


\section{The importance of pore throats in controlling the permeability of magmatic foams}

$14{ }^{4}$ Previously with: Laboratory for Synchrotron Radiation, Swiss Light Source, Paul Scherrer Institut, 155232 Villigen, Switzerland

16 Currently: Department of Earth Sciences, University of Toronto, 22 Russell St., Toronto, M5S 3B1

$17{ }^{6}$ Department of Chemical Engineering, McGill University, 3610 rue University, Montreal, QC H3A 18 0E5, Canada.

$19{ }^{7}$ School of Earth and Environmental Sciences, University of Manchester, Manchester, U.K.
Corresponding author: don.baker@mcgill.ca

1-514-398-7485
BUVO-S-18-00171

Revised 3 May 2019 


\section{Abstract}

45 Foam formation during vesiculation of hydrous magmatic melts at 1 atm was studied in situ by

46 synchrotron X-ray tomographic microscopy at the TOMCAT beamline of the Swiss Light Source

47 (Villigen, Switzerland). Four different compositions were studied; basaltic, andesitic, trachyandesitic

48 and dacitic hydrous glasses were synthesized at high pressures as starting materials and then laser

49 heated on the beamline. The porosity, bubble number density, size distributions of bubbles and pore

50 throats, as well as the tortuosity and connectivity of bubbles in the foams, were measured in three

51 dimensions based on tomographic reconstructions of sample volumes. The reconstructed volumes

52 were also used in lattice-Boltzmann simulations to determine viscous permeabilities of the samples.

53 Connectivity of bubbles by pore throats varied from $\sim 100$ to $10^{5} \mathrm{~mm}^{-3}$, and for each sample correlated

54 positively with porosity and permeability. Although permeability increased with porosity, the

55 relationship is complex; consideration of the results of this and previous studies of the viscous

56 permeabilities of aphyric and crystal-poor magmatic samples demonstrated that at similar porosities the

57 permeability could vary by many orders of magnitude, even in similar composition samples. More

58 than $90 \%$ of these permeabilities are bounded by two empirical power laws, neither of which identifies

59 a percolation threshold.

60 Comparison of the permeability relationships from this study with previous models (Degruyter et al.

61 2010; Burgisser et al. 2017) relating porosity, characteristic pore-throat diameters and tortuosity

62 demonstrated good agreement. However, modifying the Burgisser et al. (2017) model by using the

63 maximum measured pore-throat diameter, instead of the average diameter, as the characteristic

64 diameter produced a model that reproduced the lattice-Boltzmann permeabilities to within 1 order of

65 magnitude. Measured correlations between the average bubble diameter and the maximum pore-throat

66 diameter as well as between porosity and tortuosity in our experiments produced relationships that 
67 allow application of the modified Burgisser et al. model to predict permeability based only upon the

68 average bubble diameter and porosity. The experimental results are consistent with previous studies

69 suggesting that increasing bubble growth rates result in decreasing permeability of equivalent porosity

70 foams. The effect of growth rate on permeability is hypothesized to substantially contribute to the

71 multiple orders-of-magnitude variations in the permeabilities of natural magmatic samples at similar

72 porosities.

73 Keywords: magmatic foam, permeability, bubble and pore throat sizes, bubble connectivity, 74 synchrotron X-ray tomography

76 Introduction

77 The competition between magma expansion due to gas exsolution and expansion, and gas loss created

78 by vesiculation in volcanic conduits, exerts a significant control on the explosivity of volcanic

79 eruptions (e.g., Sparks 2003; Spieler et al. 2004, Mueller et al. 2005, 2008). This competition is

80 profoundly influenced by the permeability of the magmatic foam (a mixture of gas-filled bubbles and

81 melt that may contain crystals and if quenched would produce a scoria or a pumice). Relatively

82 impermeable magmas can lead to violent eruptions, whereas permeable ones may not (Sparks 2003;

83 Mueller et al. 2005, 2008). Understanding the development of porosity, $\Phi$, and permeability, k, during

84 magma vesiculation is one of the keys to quantitative modeling of volcanic processes that hold the

85 promise of a better understanding of volcanic eruptions and their precursors (Fagents et al. 2013). Due

86 to the significance of permeability, many studies characterized the porosities and permeabilities of

87 natural samples and experimental run products and demonstrated orders-of-magnitude differences in

88 permeability at similar porosities (e.g., Klug and Cashman 1996; Saar and Manga 1999; Blower 2001;

89 Rust and Cashman 2004; Mueller et al. 2005, 2008; Bouvet de Maisonneuve et al. 2008; Wright et al. 
90 2009; Degruyter et al. 2010; Bai et al. 2010; Polacci et al. 2014; Farquharson et al. 2015; Kushnir et al.

91 2016; Lindoo et al. 2016; Burgisser et al. 2017).

92 The structural details of porous media, such as tortousity and the size of the bubbles and pore throats

93 that connect them have long been known to significantly influence permeability (Carman 1937; Archie

94 1942). Polacci et al. (2008) suggested that "a few large vesicles, exhibiting mostly irregular, tortuous,

95 channel-like textures" in scoria from Stromboli volcano (Italy) were the preferential pathways used for

96 gas escape from the magma. Degruyter et al. (2010) and Burgisser et al. (2017) both demonstrated that

97 the tortuosity of the sample and the characteristic diameter of the pore throats played significant roles

98 in controlling magmatic permeability. These publications demonstrated that the size of the bubbles, the

99 pore throats that connect them, and the ways in which bubbles are interconnected (tortuosity and either

100 in a series or parallel configuration) are significant controls on gas transport in magmatic systems.

101 Here we report results of a series of high-temperature, in situ X-ray tomographic microscopy

102 experiments studying the development of crystal-free, vesiculating samples of silicate melt at 1 atm.

103 Although in the experiments bubbles are formed during heating at constant pressure (rather than during

104 decompression at approximately constant temperature in volcanic systems), the development of the

105 interconnections between bubbles provides important information on the formation of magmatic foams

106 and development of their permeability. Four melt compositions were studied: a mid-ocean ridge basalt

107 (MORB), a trachyandesite, an andesite, and a dacite The viscous permeabilities of the foams were

108 determined by using tomographic reconstructions of sample volumes as the input for lattice-Boltzmann

109 simulations of fluid flow (Hill et al. 2001; Hill and Koch 2002). We concentrated on the viscous

110 permeability $\mathrm{k}_{1}$, and the applicability of the Carman-Kozeny equation to magmatic foams (Carman

111 1937). Although we have not investigated the inertial permeabilities, $\mathrm{k}_{2}$, in our samples, relationships

112 between viscous and inertial permeability have been previously determined (e.g., Rust and Cashman 
113 2004; Yokoyama and Takeuchi 2009; Bai et al. 2010; Polacci et al. 2014; Burgisser et al. 2017). Most

114 recently Zhou et al. (2019) proposed a universal power-law equation relating viscous and inertial

115 permeabilities for all geologic porous media with parameters equivalent to those previously published

116 by Polacci et al. (2014) for volcanic samples. Thus, knowledge of the viscous permeability allows

117 calculation of the inertial permeability using the relationships in Polacci et al. (2014) and Zhou et al.

118 (2019).

119

120 Methods

121 Hydrous glass preparation

122 Samples of MORB, trachyandesite, andesite, and dacite were chosen for these experiments (Table 1).

123 The MORB is a dredge haul sample graciously donated by C. Langmuir; the trachyandesite is a scoria

124 from the 2010 eruption of Eyjafjallajökull, Iceland, and the andesite and dacite compositions were from

125 Atka Island, Alaska, USA. Each sample was ground to less than $50 \mu \mathrm{m}$ in diameter and dried at $110{ }^{\circ} \mathrm{C}$

126 before use. Approximately $70 \mathrm{mg}$ of powder plus distilled water were loaded into $3 \mathrm{~mm}$ diameter Pt

127 capsules and welded closed in a water bath without volatile loss. Water concentrations are based upon

128 the water added to the capsules and was $3 \mathrm{wt} \%$ in successful experiments with MORB, trachyandesite

129 and dacite. The only successful experiment with the andesite contained 5 wt $\%$ water. The rock plus

130 water mixtures were melted above their liquidi in a piston-cylinder apparatus at a temperature of 1250

$131{ }^{\circ} \mathrm{C}$ or $1200{ }^{\circ} \mathrm{C}$ (the trachyandesite only), and a pressure of $1.0 \mathrm{GPa}$ for a duration of $2 \mathrm{~h}$ or of $1 \mathrm{~h}$ (again

132 only the trachyandesite) in $19.1 \mathrm{~mm} \mathrm{NaCl}$-pyrex assemblies (Baker 2004) and quenched isobarically.

133 Subsamples with volumes of approximately $\sim 1$ to $2 \mathrm{~mm}^{3}$ of these crystal-free glasses were used for the

134 synchrotron X-ray tomographic microscopy experiments. 
137 In situ synchrotron X-ray tomographic microscopy was performed at the TOMCAT beamline of the 138 Swiss Light Source at the Paul Scherrer Institut (Villigen, Switzerland) using a laser-based heating 139 system (Fife et al. 2012) and the ultra-fast endstation (Mokso et al. 2010, see Mokso et al. 2017 for 140 current capabilities of the ultra-fast endstation that differ from the description below and allow more 141 rapid acquisition of images than possible when this study was performed). The laser system comprises

142 two, class four diode lasers of $980 \mathrm{~nm}$ wavelength on opposite sides of, and $40 \mathrm{~mm}$ away from, the 143 sample; these each provide up to $150 \mathrm{~W}$ of power to heat the sample. A pyrometer was used to 144 measure the temperature. The ultra-fast endstation incorporated a pco.DIMAX camera, which acquires 145 and transfers data orders of magnitude faster than traditional CCD technology (Mokso et al. 2010). To 146 reach the highest possible temperature, the lasers were pointed just below the sample holder on the 147 zirconia rod that connected the sample holder to the rotation stage below. The temperature was 148 increased until it reached approximately $600{ }^{\circ} \mathrm{C}$ at which point the sample was lowered into the laser 149 beams. Samples were then heated at either $1^{\circ} \mathrm{C} \mathrm{s}^{-1}$ or $6^{\circ} \mathrm{C} \mathrm{s}^{-1}$ to the maximum temperature of the 150 experiment, resulting in sample vesiculation and creation of a silicate foam under open-system 151 conditions such that the sample was free to expand and exsolved gas escaped the system. Initially a 152 programmed heating rate of $\sim 6^{\circ} \mathrm{C} \mathrm{s}^{-1}$ was chosen as the best compromise between instantaneous 153 heating of the sample and the need for bubbles to grow slowly enough to be successfully imaged. Due 154 to many experimental failures, a slower programmed heating rate of $\sim 1{ }^{\circ} \mathrm{C} \mathrm{s}^{-1}$ was found to produce 155 more successful experiments (Table 2). Measurement of the time-temperature histories of the 156 experiments demonstrated that the heating rates were often $\sim 20$ percent slower than the programmed 157 ones (Table 2).

158 Data acquisition was initiated at the first visible onset of vesiculation, such as bubble formation and 
sample expansion. During data acquisition, samples reached a maximum temperature between 950

$160{ }^{\circ} \mathrm{C}$ and $\sim 1200{ }^{\circ} \mathrm{C}$. Polychromatic X-rays were filtered to $5 \%$ power, generating 3 ms exposure times, 161 and 701 projections were captured over an angular range of 180 degrees during continuous rotation.

162 The microscope used for these scans incorporated a specially designed, high numerical aperture 163 objective lens with four-fold magnification. This corresponded to a $2.89 \mu \mathrm{m} \times 2.89 \mu \mathrm{m}$ pixel size and 164 a $5.83 \mathrm{~mm} \times 5.83 \mathrm{~mm}$ field of view. The optics were coupled to a LuAG:Ce $100 \mu \mathrm{m}$ thick scintillator screen. Reconstructions were performed using a modified GRIDREC algorithm (Dowd et al. 1999;

166 Rivers and Wang 2006; Marone and Stampanoni 2012) coupled with Parzen filtering of the sinograms.

167 Many bubble-growth experiments were performed, but only a few were successful. The most

168 significant problem was image blurring due to sample motion caused by rapid vesiculation and bubble

169 growth that rendered the tomographic reconstructions useless for this study. Other problems were 170 samples that failed to heat to temperatures high enough to vesiculate (which included all rhyolitic 171 samples investigated) and samples that cracked into small pieces during heating.

172 Of the 62 experiments performed, only one dynamic experiment on the andesitic composition, one on 173 the trachyandesitic composition, and one on the dacitic composition yielded 3D reconstructions that 174 could be used to extract quantitative data. Bubble growth in all dynamic experiments on the MORB 175 composition was so rapid and the motion artifacts so severe that no successful reconstructions were 176 made. However, the final steps of 4 experiments on the MORB composition were successfully imaged 177 as bubble growth slowed or stopped due to gas loss from the sample. These experiments span much of 178 the range of porosities measured in the successful tomographic scans on the andesite, trachyandesite, 179 and dacite, and allow comparison between the four different melt compositions. Even the successful 180 experiments contained some image artefacts due to sample movement during bubble growth. These 181 aretefacts were avoided during the sample analysis discussed below. 


\section{Image analysis and quantification}

184 The bubble distributions in the samples were not homogeneous because of thermal gradients in the

185 laser furnace. Thus, only representative central portions of the samples, far from their edges and the

186 capsule walls, were analyzed, and the measurements reported are not representative of the entire

187 sample, but only of the volume investigated. The tomographic reconstructions were inspected with

188 ImageJ and subvolumes from the most vesicular portions of the samples were chosen; in most cases

189 they were $256 \times 256 \times 256$ voxels in volume (Fig. 1a-c). Because these volumes were too large for

190 lattice-Boltzmann determination of their permeability (discussed below) representative subvolumes of

$191370 \times 370 \times 370 \mu \mathrm{m}^{3}\left(128 \times 128 \times \square 128\right.$ voxels, trachyandesite EFJ-8a), or $462 \times 462 \times 462 \mu \mathrm{m}^{3}(160$

$192 \times 160 \times 160$ voxels, andesite DRB2012-2a, dacite DRB2012-6e-8, -9, -10, MORB DRB2012-7a-2, -3,

193 -cf $)$ or $578 \times 578 \times 578 \mu \mathrm{m}^{3}(200 \times 200 \times 200$ voxels, dacite DRB2012-6e-07, MORB DRB2012-7f-

194 10) were used for all quantitative analyses with the Pore $3 D$ software library (Brun et al. 2010;

195 Zandomeneghi et al. 2010). An edge-preserving smoothing filter (Tomasi and Manduchi 1998) was

196 applied followed by a 3D, manually selected, global fixed threshold to separate pore space from glass.

197 This segmentation process was adopted for all datasets.

198 The vesicularity was computed as the number of voxels belonging to the pore space with respect to the 199 total number of voxels in the object. Objects, bubbles and pore throats, were not counted unless their

200 size was greater than two voxels, where each voxel was $2.89 \times 2.89 \times 2.89 \mu \mathrm{m}^{3}$ in volume. Baker et

201 al.'s (2011) study of the reproducibility of porosity measurements using X-ray microtomography

202 demonstrated that the precision of vesicularity measurements is approximately 0.01 . We expect similar

203 uncertainties for pore throats because the same techniques were used for measurements of vesicles and

204 pore throats. The interconnected porosity was determined from the same images using the 
205 ObjectCounter3D plugin in ImageJ (https://imagej.net/3D_Objects_Counter).

206 A family of descriptors based on skeleton analysis (Lindquist and Lee 1996) was applied to derive

207 bubble number and pore throat number density as well as bubble- and pore throat-size distributions. As

208 in our previous research (Baker et al. 2012), the skeletonization algorithm of Brun and Dreossi (2010)

209 was applied. Each bubble diameter and the minimum thickness of each pore throat was computed using

210 the concept of a maximal inscribed sphere, which was moved through the pore throat along the

211 skeleton to find its minimum diameter (Hildebrand and Rüegsegger 1997). The skeletonization

212 algorithm used in this study offers a tuning parameter to control the amount of branches in the output

213 skeleton. Figures 1d \& e present the differences between a case where only the most significant (and

214 larger) interconnects are considered and a case where the smallest interconnects are considered and a

215 skeleton branch is added to the skeleton network for each of them. This parameter is tuned to select the

216 maximum number of branches in this study.

217 The geometrical determination of bubbles and throats is difficult because there is no unambiguous

218 geometrical definition of where a bubble ends and a connecting channel begins. Conceptually, the

219 skeleton nodes correspond to bubbles, and the branches of the pore-space skeleton correspond to the

220 channels (or paths) connecting the bubbles. However, a typical pore/node correction has to be applied

221 because several skeleton nodes may occur in the same bubble body (Lindquist 2002), as can be seen in

222 Figures 1d and 1e. In this work, a criterion based on the isotropic inflation of spheres centered on each

223 node was used. If two or more spheres overlap, they are considered part of the same bubble. The

224 number of identified bubbles is actually the number of independent clusters of overlapping inflated

225 bubbles. The amount of inflation can be controlled and acts as a parameter for the merging criterion.

226 The steps in this process are graphically shown in the example presented in Figures 1d-1h. An

227 insufficient inflation value overestimates the number of pores, while the maximum inflation to fill the 
228 entire bubble might underestimate this value if several spurious branches result after skeletonization.

229 In this work a level of inflation equal to $85 \%$ of the maximum was used. Variations around this value

230 were also considered, and it was found that this parameter only weakly affects the computed values of

231 the bubble and pore throat numbers and sizes, as seen in Figures 1f \& $\mathrm{g}$ that present two examples with

232 different levels of inflation.

233 Some concerns still remain for the throat size determinations; while very short branches are usually

234 disregarded, because some maximal spheres centered at the skeleton nodes may completely enclose the

235 short branches, incorrect channels may still be considered. Practically, this means that the throat-size

236 distribution may present some large-valued outliers due to the consideration of branches that may not

237 represent physical channels.

238 The final assessment of bubble size is based on the diameter of the maximal inscribed sphere placed at

239 the center of mass of the cluster of the overlapping inflated bubbles (Fig. 1h ). The bubble number

240 density was calculated by dividing the number of inscribed spheres (bubbles) identified after

241 skeletonization by the investigated volume. The uncertainties in the numbers of bubbles and of pore

242 throats can be estimated as the square root of the number of each object type counted by application of

243 Poisson statistics.

244 The connectivity, $\beta$, is a standard topological property that measures the number of interconnections (in

245 this study pore throats) between objects (in this case bubbles). Connectivity analysis of tomographic

246 reconstructions was pioneered in studies of bone structure (Odgaard and Dundersen 1993) and of

247 porous media (Thovert et al. 1993). Following these two publications we calculate the connectivity

248 per unit volume ( $\mathrm{mm}^{3}$ in this study), $\beta$ or connective density, as done by Odgaard and Gundersen

249 (1993) using: 
251 This topological measure may yield both negative and positive values per unit volume. As an

252 illustration, consider a simplified example of 4 bubbles in a volume of $0.5 \mathrm{~mm}^{3}$. In the case of no

253 interconnections, or pore throats, between the bubbles, the value of $\beta$ is $-6 \mathrm{~mm}^{-3}$. In cases where only

254 single connections are allowed between bubbles (i.e., two pore throats cannot connect the same two

255 bubbles), a system with two interconnections yields a $\beta$ value of $-2 \mathrm{~mm}^{-3}$, if there are three

256 interconnections $\beta=0 \mathrm{~mm}^{-3}$, and if there are four $\beta=2 \mathrm{~mm}^{-3}$. Thus, a $\beta$ value of $0 \mathrm{~mm}^{-3}$ is the

257 minimum threshold at which the pore throats interconnect the bubbles in this example. This threshold

258 value can exceed 0 if we relax the constraint that bubbles can only be connected by a single pore throat

259 or that all pore throats must interconnect bubbles within the volume of interest. Following basic

260 statistical rules, the uncertainties in the values of $\beta$ are calculated from the uncertainty in the number of

261 bubbles, $\delta$ bubbles, and the uncertainty in the number of pore throats, $\delta$ pore throats, and the sample

262 volume by

$$
\delta \beta=\frac{\sqrt{(\delta \text { bubbles })^{2}+(\delta \text { pore throats })^{2}}}{\text { sample volume }} .
$$

264 Tortuosity in this research is defined as the average distance a particle would travel between two

265 opposite sides of the sample by the pore network divided by the Euclidean distance between the

266 opposite sides. The MATLAB® code TORT3D (Al-Raoush and Madhoun 2017) was used for all

267 tortuosity measurements. The tortuosity was determined in three orthogonal directions and then

268 averaged; the average values are reported in Table 2.

269 The 3D visualization of reconstructed and analyzed volumes was obtained by the commercial software

270 VGStudio Max 2.0 (Volume Graphics). 
273 Because of the dynamic nature of the experiments and the collapse of the samples with loss of

274 vesicularity near to, or at, their termination, sample permeabilities could not be measured directly.

275 Instead, lattice-Boltzmann modeling of permeabilities was performed using a modified version of an

276 established lattice-Boltzmann code (Hill et al. 2001; Hill and Koch 2002). Details of the permeability

277 modeling applied to tomographic reconstructions can be found in Bai et al. (2010), in which the

278 accuracy of the viscous permeabilities calculated by modeling was directly compared against measured

279 permeabilities and shown to typically be within a factor of 11 for porosities from 0.05 to 0.87 .

280 Bai et al. (2010) demonstrated that the ratio of the tomographic reconstruction resolution (voxel edge

281 length) to the lattice size (edge length of subvolume) and the simulation size could influence the

282 calculated permeability. The resolution-to-lattice-size ratios used in this study are in the range where

283 Bai et al. (2010) demonstrated that the value of the ratio had no effect on the calculated permeability.

284 Bai et al. (2010) found that decreasing the lattice size below $762 \mu \mathrm{m}$ increased the calculated

285 permeability by a factor of less than 20. Analysis of Bai et al.'s (2010) results indicates that using the 286 smallest lattice size in this study $(370 \mu \mathrm{m})$ may, at most, overestimate the permeability by a factor of

2873 for low porosity samples. The other, larger, simulations used in this study are expected to have 288 uncertainties less than $20 \%$ (Bai et al. 2010).

289 Permeabilities in the three orthogonal directions of each subvolume were calculated. Because the 290 maximum difference between the lattice-Boltzmann permeabilities measured in three orthogonal 291 directions of the samples was less than a factor of 2, we concluded that there was no significant 292 variation in the permeability as a function of direction and report the average permeability values for 293 each sample in Table 2. This lack of orientation effects supports the modeling of our samples as 294 random networks of bubbles interconnected by pore throats. 


\section{Bubble growth during isobaric heating versus isothermal decompression}

297 Experiments in this study were performed by isobaric heating at atmospheric pressure because a high-

298 pressure furnace was not available on the TOMCAT beamline. The resulting time-temperature-

299 pressure path in these experiments is distinctly different from bubble formation during near-isothermal 300 decompression in natural systems and in many experiments (e.g., Burgisser and Gardner 2004; Lindoo 301 et al 2016, 2017; Mueller et al. 2005; Spieler et al. 2004; Takeuchi et al. 2009).

302 Although bubble nucleation and growth during isothermal decompression and isobaric heating 303 experiments are both driven by supersaturation of the melt with a volatile, the viscosity of the melt 304 increases during isothermal decompression (due to water loss to the bubbles) and may either increase 305 (due to water loss) or decrease (due to increasing temperature) during isobaric heating. For example, in

306 these experiments the andesitic melt begins vesiculation at $900{ }^{\circ} \mathrm{C}$ with a water concentration of $5 \mathrm{wt} \%$ 307 and a viscosity of $\sim 750 \mathrm{~Pa}$ s (calculated following Giordano et al. 2008). If this melt lost all its water

308 by the end of the experiment at $1100{ }^{\circ} \mathrm{C}$, the viscosity would be $\sim 6700 \mathrm{~Pa}$ s. Although it is difficult to 309 estimate the water concentration in the melt during vesiculation, we estimate that approximately $1 \mathrm{wt} \%$ 310 water remains in the melt at $\sim 1000{ }^{\circ} \mathrm{C}$, which would yield a melt viscosity $\sim 3100$ Pa s. During these

311 experiments the diffusivity of water in the melt also is controlled by a combination of heating and

312 dehydration. Using the equations of $\mathrm{Ni}$ and Zhang (2018), the water diffusivity at the start of

313 vesiculation is expected to be $\sim 3 \times 10^{-13} \mathrm{~m}^{2} \mathrm{~s}^{-1}$, decreasing to $\sim 1 \times 10^{-14} \mathrm{~m}^{2} \mathrm{~s}^{-1}$ when water is lost. On

314 the contrary, for isothermal decompression at $1100{ }^{\circ} \mathrm{C}$ of the same melt composition, the viscosity 315 would increase from $32 \mathrm{~Pa} \mathrm{~s}$ at the start of vesiculation to $\sim 6700 \mathrm{~Pa}$ s if all of the water is exsolved

316 from the melt, and the water diffusivity would decrease from $\sim 3 \times 10^{-12} \mathrm{~m}^{2} \mathrm{~s}^{-1}$ to $\sim 1 \times 10^{-14} \mathrm{~m}^{2} \mathrm{~s}^{-1}$.

317 These differences in the history of the melt viscosity and diffusivity will influence the rates of bubble 
318 growth and coalescence of neighboring bubbles. Following Navon and Lyakhovsky (1998) the radius

319 of an individual bubble during its initial stages of growth will be significantly affected by the melt

320 viscosity:

$$
r \propto \exp \left[\frac{\Delta P}{4 \eta} t\right]
$$

322 where $\mathrm{r}$ is the bubble radius, $\Delta \mathrm{P}$ is the supersaturation pressure, $\eta$ is the melt viscosity, and $\mathrm{t}$ is the time.

323 As the bubble grows and supersaturation decreases, the bubble radius is described by a law containing

324 the square root of the product of the volatile diffusivity in the melt and time (Equation 36 of Navon and

325 Lyakhovsky 1998). Melt viscosity also exerts control on the time necessary for interacting bubble

326 walls to fail and coalescence to begin, $\tau_{\mathrm{df}}$ :

$$
\tau_{d f}=\frac{3 \eta r^{2}}{4 \Delta P h_{\min }^{2}}
$$

328 where $h_{\min }$ is the critical thickness at which the walls fail (Navon and Lyakhovsky 1998). Equations 3 329 and 4 demonstrate that the rates of bubble growth and coalescence during the early stages of isobaric

330 heating experiments should be slower than those in isothermal decompression experiments because of

331 the higher viscosities and lower water diffusivities in the melt early in the isobaric heating experiments.

332 However, as both types of experiments reach the end of bubble growth and water loss, the rates at

333 which bubble growth and coalescence occur should converge.

334 Both isothermal decompression and isobaric heating experiments are expected to produce similar, 335 random bubble, or foam, structures, due to the stochasticity in both the location and timing of bubble 336 nucleation in the melts. Because of the higher viscosities and lower diffusivities of isobaric heating 337 experiments, the vesicularity and interconnectivity of bubbles (due to coalescence) may be expected to 338 be smaller than in isothermal decompression experiments of similar, short durations (Equations 3 and 339 4). Because of these differences between isothermal decompression and isobaric heating experiments 
340 we did not study bubble growth and coalescence rates, but instead concentrated on the development of

341 porosity and permeability of the foams, while fully recognizing that the values we determined may be 342 minimal ones.

343 Despite the differences between isothermal decompression and isobaric heating experiments, we can

344 make a comparison between the rates of magma ascent and the rates of degassing during isobaric

345 heating experiments by dividing the melt supersaturation pressure (Table 2) by the duration of the

346 isobaric heating (Table 2) to yield equivalent decompression rates of approximately 0.1 to $2 \mathrm{MPa} \mathrm{s}^{-1}$.

347 Although these equivalent decompression rates are only rough approximations, the low values are

348 similar to decompression rates found by Ferguson et al. (2016) for eruptive products of Kilauea

349 volcano and the high values to decompression rates found by Humphreys et al. (2008) for the May 18, 3501980 plinian eruption of Mt. St. Helens.

\section{Results}

\section{Visual observations}

354 Bubble growth in the samples occurred rapidly at temperatures above $600{ }^{\circ} \mathrm{C}$. Typical bubble 355 nucleation and growth can be seen in Figure 2 and Supplemental Movie 1 of andesitic sample 356 DRB2012-2a, which was heated from $600{ }^{\circ} \mathrm{C}$ to $1100{ }^{\circ} \mathrm{C}$ over a period of $100 \mathrm{~s}$ (Table 2).

357 In the absence of nucleation delay, bubble growth is presumed to occur once the sample temperature 358 exceeds that of the glass transition. The onset of the glass transition temperatures for the samples 359 studied can be estimated using the results of Giordano et al. (2005). The glass transition given in 360 Giordano et al. (2005) for basaltic, trachytic and dacitic compositions determined at their most rapid

361 heating rate of $0.333 \mathrm{~K} \mathrm{~s}^{-1}$ and water concentrations up to $2.5 \mathrm{wt}$. \% were fit by a power-law, which was 
362 chosen because power-laws describe the effect of water addition on melt viscosity (e.g., Shaw 1972).

363 The power-law fit reproduces the calibrating data to within a maximum of $\sim 50 \mathrm{~K}$, as well as

364 reproducing the anhydrous andesite glass transition temperature of Neuville et al. (1993) that was not

365 used in the calibration. We estimate a minimum onset of the glass transition in our samples with 3 wt $\%$

366 water (MORB, trachyandesite, and dacite) at $460{ }^{\circ} \mathrm{C}$ and in the andesite with 5 wt $\%$ water at $440{ }^{\circ} \mathrm{C}$.

367 However our heating rates were approximately 3 to 15 times more rapid than used in Giordano et al.'s

368 experiments, thus the observed vesiculation temperatures were significantly above these minimum

369 glass transition temperatures, in the range of 616 to $900{ }^{\circ} \mathrm{C}$ (Table 2). Although these delays may be

370 attributable to a short nucleation delay, we interpret them as being due to our inability to observe the

371 onset of bubble growth in real time during the experiments because of the small size of the initial

372 bubbles. This inability to see the earliest bubbles made it difficult to measure samples at low porosities

373 and only a few data at these conditions were obtained (Table 2).

374 Bubble growth was initially observed as a dense cloud of small bubbles that grew into larger, easily

375 discernible, bubbles that rapidly became interconnected (Fig. 2). Typically, these early growth rates

376 were so rapid that they were blurred in the tomographic images, so meaningful quantitative

377 measurements could not be made.

378 Visual inspection of the tomographic reconstructions revealed that early bubbles vary from ellipsoidal

379 to sub-spherical, but within seconds all evolve into sub-spherical to spherical shapes. Bubble growth in

380 all experiments on the basaltic composition was too rapid to quantitatively analyze those samples

381 before termination of the experiment. However, in four cases we extracted limited data from the

382 different stages of foam development (Table 2). Bubbles coalesced and typically grew to a maximum

383 size, creating a foam with thin-walled bubbles. If the sample was not immediately quenched, the foam

384 contracted and underwent partial collapse, and in some cases collapse occurred before the termination 
385 of the experiment. This behavior is attributed to the loss of volatiles, either through failure of the

386 bubble walls or diffusion through them.

387 Not every tomographic reconstruction set could be used for all the quantitative analyses presented in

388 the Methods section. In some cases, permeabilities could not be determined (e.g., DRB2012-6e-07)

389 because of the large computer memory required to resolve the surface area of the interconnected

390 bubbles.

392 Bubble number density (BND), bubble size distribution (BSD), and pore throat size distribution (PTD)

393 Table 2 presents a summary of our quantitative measurements, and the sizes of all bubbles and pore

394 throats measured in the studied samples are provided as supplementary material (Table S1). All

395 measurements reported in this study were made on volumes far from the edges of the samples and that

396 displayed no visual evidence of anisotropy. The typical distance of the analyzed volume from the

397 sample edges was approximately $500 \mu \mathrm{m}$. Because of the small sample volume, in some cases the

398 number of bubbles and pore throats, as well as their range in size, are small (Table S1). In all but one

399 case for the dacitic composition, DRB2012-6e-10, the largest bubble in the investigated volume was

400 less than $6 \%$ of the sample volume. In DRB32012-6e-10 the largest bubble was $31 \%$ of the volume.

401 In general, in each sample the bubble number densities tend to increase with porosity up to a maximum

402 and decrease at higher porosity (Figs. 3-6, Table 2), although between any two steps the BND can

403 decrease (Table 2). Increasing BNDs are attributed to continuous nucleation and decreasing BNDs to

404 bubble coalescence.

405 The measured bubble size and pore throat size distributions of the experiments are presented in Figures

406 3-6. Each figure presents a volume-normalized histogram of the sizes of either the bubbles or the pore 
407 throats. None of the distributions are Gaussian, but instead often display "long tails" to large bubble 408 and throat sizes, a characteristic indicative of power-law distributions (Newman 2005). Although the 409 bubble-size and throat-size distributions can be fit with power-laws, this was not done in this study due 410 to the small size range in each distribution that results in large uncertainties in the calculated power-law 411 exponent. Despite evidence that the sizes are power-law distributed, and therefore their means and 412 standard deviations do not have the same significance as in Gaussian distributions (Newman 2005), 413 each panel of each figure provides the mean diameter and the standard deviation of either the bubble 414 diameter or pore throat diameter to allow comparison of the measurements with previous studies (e.g., 415 Burgisser et al. 2017). The relative standard deviations about the mean bubble sizes and pore throats 416 are in most samples greater than $25 \%$ and often near $50 \%$. These large standard deviations are 417 consistent with the non-Gaussian distributions of bubble and pore throat sizes (Newman 2005).

418 Bubbles measured in the basaltic composition reach a size of approximately $460 \mu \mathrm{m}$ in diameter, and 419 the BNDs are in the thousands per cubic millimeter (Fig. 3a, Table 2). The two samples with the 420 lowest porosities $(0.52$ and 0.50$)$ are from the same experiment and demonstrate that with time the 421 number of small bubbles in the 5 to $10 \mu \mathrm{m}$ range increases, which indicates continuing bubble 422 nucleation and growth, as does that of larger bubbles in the 40 to $70 \mu \mathrm{m}$ range, which provides 423 evidence of continued growth. The disappearance of bubbles in the size range of 55 to $60 \mu \mathrm{m}$ of the 424 second sample $(\Phi=0.50)$ is interpreted as a consequence of bubble coalescence, and its lower porosity 425 is probably due to bubble collapse that occurred between these two heating experiments (Table 2).

426 Comparison of the average bubble size in each experiment does not indicate any significant trend with 427 porosity (Fig. 3a).

428 The number of pore throats in the basalt varies from about $6.40 \times 10^{3} \mathrm{~mm}^{-3}$ at the lowest porosity to 429 approximately $7.22 \times 10^{4} \mathrm{~mm}^{-3}$ at the highest porosity. The two low-porosity $(0.50$ and 0.52$)$ 
measurements of pore throats experiment demonstrate only moderate increases in the number and size

431 of pore throats (Fig. 3b, Table 2 ). The experiment with 0.55 porosity contains one pore throat that is

432 anomalously larger then the next largest one (Fig. 3b), and our experimental techniques for measuring

433 the pore diameter may produce large-valued outliers in the pore throat size distributions. Whereas we

434 cannot completely discard the possibility that the largest pore throat in this sample is an artefact, we

435 think that size difference between the largest pore throat and the others in the distribution (Fig. 3b) is

436 not sufficiently large to discard the measurement. Because the basaltic results for the two highest

437 porosity measurements $(0.55$ and 0.73$)$ are from different experiments, it is difficult to investigate the

438 evolution of BSD and PTD with increasing porosity. Nevertheless, if these two samples are interpreted

439 as an evolutionary trend, there appears to be an increasing number of pore throats with increasing

440 porosity (Fig 3b, Table 2).

441 In contrast to the basaltic experiments, all of the andesite data (Fig. 4a, b, c, Table 2) were collected

442 from the same experiment; however the location of the sample volume quantified varied slightly

443 between different time steps in order to choose the most representative volumes near the center of the

444 samples and to avoid volumes containing motion artefacts and/or anomalously large or small bubbles.

445 The maximum bubble size was between 215 and $220 \mu \mathrm{m}$ (Fig. 4c). All andesite BSDs are dominated

446 by bubbles in the 5 to $20 \mu \mathrm{m}$ size range (Fig. $4 \mathrm{a}, \mathrm{b}, \mathrm{c}$ ). The large number of small bubbles in all but the

447 final porosity is interpreted to reflect a process of continuing nucleation and growth of new bubbles.

448 The appearance of larger bubbles, greater than $40 \mu \mathrm{m}$, at porosities above 0.41 is caused by the

449 expansion of gas within them during heating, and by coalescence. Evidence of coalescence is seen in

450 the decreasing number of intermediate-sized bubbles with the appearance of larger bubbles in the

451 highest porosity samples (cf., Gaonac'h et al. 1996). The PTD distributions demonstrate the growth of

452 throat sizes and number with increasing porosity (Figs. 4d, e, f). The largest pore throat size grows 
453 from $10-15 \mu \mathrm{m}$ to a maximum of $50-55 \mu \mathrm{m}$. In comparison to the basalt, there are fewer pore throats 454 greater than $30 \mu \mathrm{m}$.

455 The BSDs for the trachyandesite at the two highest porosities display broad peaks in the distribution 456 without long tails to large bubble sizes seen in some of the andesite and basalt BSDs (Fig. 5a, Table 2).

457 The lowest porosity sample had only a single bubble and no pore throats in a volume of $0.0506 \mathrm{~mm}^{3}$

458 (Fig. 5a, b, Table 2). The maximum bubble measured was in the 95 to $100 \mu \mathrm{m}$ size class. Although no 459 bubbles less than $10 \mu \mathrm{m}$ were observed at a porosity of 0.61 , one appears at $\Phi=0.64$, suggesting 460 continuing bubble nucleation. Bubble coalescence between the same two porosities is indicated by the 461 decreasing number of bubbles with sizes between 10 and $75 \mu \mathrm{m}$ and increasing numbers of larger-sized 462 bubbles in the same sized volume (Fig. 5a). The PTDs of the trachyandesite show a growth in the 463 density of pore throats between 0.30 and 0.61 porosity followed by a decline in densities between 0.61 464 and 0.64 porosity with an increase in the number of larger pore throats (Fig. 5 b.).

465 The dacite BSDs demonstrate the growth of larger bubbles with increasing porosity, up to a maximum 466 of almost $400 \mu \mathrm{m}$ at $\Phi=0.84$ (Fig. 6a). Bubbles in the size range of $10-15 \mu \mathrm{m}$ increase in number 467 between porosities of 0.38 and 0.79 , and subsequently decrease as larger bubbles appear at higher 468 porosities, a behavior we attribute to coalescence (cf., Gaonac'h et al. 1996). The PTDs of the dacitic 469 sample demonstrate growth of larger pore throats up to a porosity of 0.87 followed by a decrease in 470 porosity at the end of the experiment to 0.84 (Fig 6b). Although the largest pore throats reach $70 \mu \mathrm{m}$, 471 the distributions are dominated by smaller pore throats. The loss of the large pore throats seen in the 472 distribution for $\Phi=0.84$ is associated with the growth of the large bubbles in this sample (Fig. 6a), 473 which are presumed to have incorporated the larger pore throats into them during growth. 
In all cases, the connected porosity is similar to the total porosity (Table 2); however the connectivity,

$477 \beta$ (Eqn. 1), varies significantly (Table 2, Supplementary Fig. 1). The $\beta$ values in each type of foam

478 increase from hundreds to thousands per cubic millimeter with increasing porosity (Table 2) up to

479 maximums in the tens to hundreds of thousands per cubic millimeter and then, with the exception of the

480 basaltic composition, decrease at higher porosities (Table 2). This trend is similar to those observed for

481 the BNDs and the PTDs (Table 2, Figs. 3-6).

482 The average coordination number (or number of interconnected bubbles surrounding a specified

483 bubble) for each porosity (Table 2) of an individual melt composition is similar and varies between $\sim 4$

484 and 6, with a few outliers reaching values near 7 (DRB2012-7c-f, DRB2012-2a-18, DRB2012-6e-10)

485 and one almost reaching 8 (DRB2012-2a-19). These average values are far below the maximum value

486 of equal-volume, deformable bubbles surrounding a central bubble (the kissing number) of 32 (Cox and

487 Graner 2004). However, the standard deviations about the average for each sample are often as large

488 as, or even larger than, the mean, and the maximum coordination numbers are often near 100 and can

489 reach almost 600 (Table 2). The bubbles with such high coordination numbers are large and

490 surrounded by a network of small bubbles. (A two-dimensional image of such a bubble can be seen in

491 Fig. 1.) Such high values of the kissing number are not inconsistent with simulations of polydisperse

492 foams that display average coordination numbers between 11 and 14, but contain some foam polyhedra

493 with coordinations approaching 100 (Kraynik et al. 2004).

494 The tortuosity, $\tau$, of the foams varies from a low of 1.09 (dacites DRB2012-06e-8 and DRB2012-6e-9)

495 to a high of 1.72 (andesite DRB2012-2a-9); however, most tortuosity values fall between 1.1 and 1.3

496 (Table 2). The relationship between increasing porosity and decreasing tortuosity in the studied

497 samples can be described by (cf., Wright et al. 2009, Degruyter et al. 2010):

$$
\tau=(1.0487 \pm 1.0201)(\Phi)^{-0.3192 \pm 0.0252},
$$


and a correlation between increasing connectivity and decreasing tortuosity was also found:

$$
\tau=(2.4376 \pm 1.1044)(\beta)^{-0.0687 \pm 0.0102} .
$$

501 Although the uncertainties in the fitting parameters are large, these equations provide useful

502 relationships between these different measures of foam properties, as will be shown below.

504 Permeability

505 The lattice-Boltzmann (LB), viscous permeabilities, $\mathrm{k}_{1}$, of the samples vary from $3 \times 10^{-15}$ to greater 506 than $5 \times 10^{-11} \mathrm{~m}^{2}$ (Table 2, Fig. 7). Note that simulations for one sample, DRB2012-6e-7 failed due to

507 its extraordinarily fine structure, so its permeability could not be determined (discussed above).

508 Another sample, EFJ08a-06, had only one bubble and its permeability also could not be determined.

509 The data sets in Figure 7 were fit with a power law because both the Carman-Kozeny relation (Carman

510 1937) and percolation theory (Stauffer and Aharony, 1994) predict a power-law relationship between

511 porosity and permeability. Formally, the percolation theory relationship is expected to be $k_{1} \sim$

$512\left(\Phi-\Phi_{c}\right)^{\mu}$, where, $k_{l}$ is the viscous permeability, $\Phi_{c}$ is the critical porosity threshold where the sample

513 becomes permeable, and $\mu$ is an exponent that depends upon the system dimensionality (Stauffer and

514 Aharony, 1994). The critical porosity threshold for monodisperse spheres in three dimensions is $\sim 0.29$

515 (Domb 1972; Lorenz and Ziff 2001); however, as discussed in detail below, the critical porosity

516 threshold is a function of the size distribution and shape of the vesicles. Permeability thresholds in

517 natural and experimental magmatic foams can vary from below 0.03 (e.g., Saar and Manga 1999; Bai

518 et al. 2010) to values in excess of 0.63 (Takeuchi et al. 2008, 2009; Lindoo et al. 2016). The critical

519 porosity threshold for any given sample is unknown, and the porosity-permeability relationships in 520 some magmatic foams have been empirically fit with a power law of the form $k_{1}=\mathrm{A} \Phi^{B}$, where $\mathrm{A}$ and 
521 B are fitting constants (e.g., Klug and Cashman 1996; Bai et al. 2010), although in some other studies

522 the estimated critical porosity threshold (typically 0.3 ) has been included in permeability-porosity

523 relationships (e.g., Saar and Manga 1999; Blower 2001; Rust and Cashman 2004, 2011).

524 Our most complete data set on the andesitic composition foam displays a power-law relationship

525 between porosity, $\Phi$, and LB permeability of $k_{1}=1.68 \times 10^{-11} \Phi^{5}$ (Fig. 8). Assuming a critical

526 porosity of 0.010 below the minimum porosity at which a LB permeability was determined, or 0.15 ,

527 produces a percolation theory power law fit of $k_{1}=5.79 \times 10^{-12}\left(\Phi-\Phi_{c}\right)^{2.1}$. At porosities up to

528 0.60, the measurements are similar to the Bai et al. (2010) fit to permeabilities of silicic rocks measured

529 by Klug and Cashman (1996). The LB permeabilities of andesitic foams at porosities greater than

$530 \quad 0.60$ diverge from Bai et al.'s (2010) fit to silicic foams leading to an order of magnitude difference

531 between the two at a porosity of 0.90 (Fig. 7).

532 The LB permeabilities of two basaltic foams at $\sim 0.50$ porosity bracket the fit to the andesitic foam but

533 the LB permeability increases to a value that is an order of magnitude greater at a porosity of $\sim 0.55$.

534 The permeability values at $\sim 0.50$ porosity (DRB2012-07a-3) may be artificially low because of bubble

535 bursting that was observed at the end of previous experiment (DRB2012-07a-2), and some of the

536 porosity and pore throats may have been lost. These measurements at 0.50 and 0.55 porosity were

537 made on two different chips of basaltic melt; this complicates the interpretation because the two

538 samples have slightly different time-temperature histories, which together with the bubble popping in

539 the sample at 0.50 porosity may might create differences in foam porosity and permeability. Thus, we

540 doubt that the factor of ten increase in permeability between $\sim 0.50$ and 0.55 porosity for these basaltic

541 samples is significant. The LB permeabilities at 0.55 and 0.73 porosity are similar to those found by

542 Bai et al. (2010) on a high-K composition basaltic foam from Stromboli, as shown by the fit to their

543 data in Figure 7. 
544 The trachyandesitic foam displays a unique behavior. At low porosities, near 0.30, its LB permeability

545 is significantly above that of the andesitic experiment, but at higher porosities the LB permeability falls

546 to values similar to the andesitic composition (Fig. 7).

547 The LB permeability of the dacitic composition at 0.79 porosity cannot be distinguished from that of

548 the similarly porous andesitic experiment, but the two higher porosity dacitic foams display

549 significantly higher LB permeabilities than expected from the trend described by the lower porosity

550 dacitic and andesitic experiments (Fig. 7).

551 The experimental results demonstrate that both average bubble sizes and pore throat sizes increase with 552 increasing vesicularity; however, the increase in pore throat sizes is less than that of the bubbles (Figs.

553 3-6). The maximum bubble sizes are observed in the dacitic composition, reaching nearly $400 \mu \mathrm{m}$ in

554 diameter, whereas the maximum bubble sizes for the basaltic, andesitic, and trachyandesitic

555 compositions are 180,220 , and $100 \mu \mathrm{m}$, respectively. The maximum throat diameters are near $100 \mu \mathrm{m}$

556 in the basaltic foam, but only $55 \mu \mathrm{m}$ in the andesitic foam, $35 \mu \mathrm{m}$ in the trachyandesitic foam, and 65 in

557 the dacitic foam. Increasing vesicularity increases connectivity and decreases tortuosity. All of these

558 changes in foam structure result in higher permeabilities that are not simply related to the melt

559 compositions investigated.

560

\section{Discussion}

562 Permeability is not a simple function of porosity

563 Porosity is often considered the primary control of permeability; in most cases increasing porosity

564 results in higher permeabilities (e.g., Fig. 7), but it has long been understood that other variables such

565 as bubble sizes, composition, connectivity, pore throat diameter, tortuosity etc. significantly influence 
566 permeability (e.g., Carman 1937; Archie 1942; Rust and Cashman 2004; Mueller et al. 2005; Bai et al.

567 2010; Degruyter et al. 2010; Polacci et al. 2012, 2014; Lindoo et al. 2016; Burgisser et al. 2017;

568 Colombier et al. 2017).

569 An obvious and significant difference in the physical properties of the studied melt compositions is

570 their viscosity (Table 1). However, a simple correlation between melt viscosity and permeability at a

571 specific porosity does not exist. The experimental results using crystal-free samples demonstrate that

572 at $\sim 0.5$ porosity basalts can have LB permeabilities similar to andesites, and at porosities approaching

5730.9 the lattice-Boltzmann permeabilities of basalts and dacites are similar (Fig. 7) despite these melts

574 displaying orders-of-magnitude differences in their viscosities (Table 1). Comparison of melt

575 viscosities with the average pore-throat diameters (Figs. 3-6) does not provide clear evidence of a

576 positive correlation between these two properties either (cf., Polacci et al. 2014).

577 Our study provides no evidence that composition alone controls the permeability-porosity relationship.

578 Connectivity also does not provide a simple predictor of permeability, as evidenced by an order of

579 magnitude difference in the lattice-Boltzmann permeability of samples with similar connectivity and

580 porosity (Table 2, Fig. 7, Supplementary Figure 1). Tortuosity also does not appear to directly

581 correlate with the porosity-permeability relations of the samples studied (Table 2). However, the

582 bubble- and pore throat-size distributions (Figs. 3 -6) suggest that larger bubbles and larger pore

583 throats play a significant role in influencing the permeabilities of magmatic foams. Before

584 quantitatively investigating the role of bubble- and pore throat sizes in controlling the permeability of

585 magmatic foams, we compare our results with those from previous studies.

\section{Porosity-permeability trends compared to previous determinations}

588 Our lattice-Boltzmann permeability determinations are compared with previous measurements of 
589 porosity and permeability from aphyric to low-crystallinity natural and experimental samples in Figure

590 8. These data were taken from published studies to illustrate the distribution of measured

591 permeabilities and porosities. The lattice-Boltzmann permeability determinations of the experiments in

592 this study are consistent with previous measurements of similar composition samples (Fig. 8), even

593 though in some cases our experimental samples are orders of magnitude smaller than natural samples.

594 At any given porosity the combined data set demonstrates that permeability can vary by orders of

595 magnitude; nevertheless, the data define significant trends as porosity increases from negligible values

596 to near 1.0 (Fig. 8).

597 Most viscous permeabilities increase from $\sim 10^{-17} \mathrm{~m}^{2}$ at porosities near 0.01 to $\sim 10^{-13} \mathrm{~m}^{2}$ at 0.20 to 0.30

598 porosity, although some rhyolites at these porosities have permeabilities of only $10^{-15} \mathrm{~m}^{2}$ (Fig. 8). At

599 porosities above $\sim 0.3$, the sphere percolation threshold (Lorenz and Ziff 2001), the permeabilities

600 continue to increase but at a slower rate than observed at lower porosities (Fig. 8). For porosities

601 between 0.5 and 0.9, the permeabilities in Figure 8 range from values as low as $\sim 10^{-14} \mathrm{~m}^{2}$ (Lindoo et

602 al. 2016) to as high as $10^{-10} \mathrm{~m}^{2}$ (Bai et al. 2010). The slow increase in permeability at porosities above

6030.3 suggests that once a permeable pathway is created, the addition of other pathways for gas transport

604 at higher porosities (as shown by higher values of $\beta$ and lower values of tortuosity) increases the

605 permeability much less significantly than the first pathway. Most lattice-Boltzmann permeabilities

606 measured in this study are near the center of the trend in Figure 8, with the exceptions of the highest

607 permeability basaltic and dacitic melts.

608

609 In general, silicic foams have lower permeabilities and mafic foams higher permeabilities, but the

610 dacitic foams with greater than 0.80 porosity measured in this study have lattice-Boltzmann

611 permeabilities similar to basaltic foams with similar porosities (Saar and Manga 1999; Bai et al. 2010). 
612 Rust and Cashman's (2004) permeabilities of rhyolite, pumice and obsidian as well as Farquharson et

613 al.'s (2015) permeabilities of pumiceous andesite demonstrate almost a two order-of-magnitude

614 variation at similar porosities (Fig. 8). Thus the influence of composition on any porosity permeability

615 relationship appears to be weak.

616 The porosity-permeability data set contains many determinations at porosities below the nominal

617 critical value for mono-disperse spheres. Each specific sample may have its own critical porosity based

618 upon the size distribution and shape of the bubbles and pore throats. Because of our lack of knowledge

619 of the critical porosity for each sample, the data were fit by empirical power laws without including a

620 critical porosity. Although the data in Figure 8 can be empirically fit with a single power law, $k_{1}=$

$6216.0 \times 10^{-12} \Phi^{4.0}$, the dispersion of the permeabilities around this average fit is orders of magnitude at

622 porosities from 0.15 to 0.90 (Fig. 8). The range of permeabilities displayed in Figure 8 also can be

623 bound by two power-law fits. The lower bound is constrained by permeability measurements below

$62410^{-14} \mathrm{~m}^{2}: k_{1}=1.5 \times 10^{-14} \Phi^{1.8}$. The upper bound is dominated by the high permeability basaltic foams

625 of Saar and Manga (1999) and of Bai et al. (2010), and can be described by a power law of $k_{1}=$

$6261.5 \times 10^{-10} \Phi^{4.0}$. More than $90 \%$ of the viscous permeability determinations in Figure 8 , including

627 those of this study, fall within the boundaries defined by these two power-laws.

628 Percolation theory can explain the great increase in permeability between 0 and 0.30 porosity. This

629 theory predicts that at about 0.29 porosity an ensemble of inter-penetrating, equivalent diameter

630 spheres that are randomly distributed in three-dimensional space will create a spanning, permeable

631 network of connected porosity (Domb 1972; Lorenz and Ziff 2001). The permeability at porosities

632 below 0.3 and the lack of permeability in some foams at porosities as high as $\sim 0.8$ (e.g., Takeuchi et al.

633 2008, 2009; Lindoo et al. 2016; Burgisser et al. 2017) may reflect the observations that the percolation

634 threshold in finite systems does not necessarily occur at a specified porosity for a non-infinite system 
635 (Stauffer and Aharony 1994; Colombier et al. 2017). Possibly the permeabilities at low porosities are 636 controlled by the percolation threshold for hard spheres, which has been determined at a porosity of 6370.1938 (Ogata et al. 2005) or 0.1990 (Ziff and Torquato 2017). Another possibility is that a non638 constant size distribution of bubbles will affect the percolation threshold. However, Consiglio et al. 639 (2003) and Ogata et al. (2005) demonstrated that the effect of the non-uniform size distributions they 640 investigated on the percolation threshold was not significant for interpenetrating spheres and hard 641 spheres, respectively. However, Burgisser et al. (2017) demonstrated with their experimental samples 642 that the separation distance between bubbles weighted by the polydispersity of the bubble sizes affected 643 the percolation threshold.

644 On the other hand, bubble shape plays a significant role in controlling the percolation threshold.

645 Garboczi et al. (1995) demonstrated that the continuum percolation threshold for randomly oriented, 646 inter-penetrating, prolate ellipsoids decreased from the porosity value for spheres of 0.29 (e.g., Lorenz 647 and Ziff 2001) to 0.26 for an aspect ratio of 2 , to 0.18 for an aspect ratio of 4 , to 0.09 for an aspect ratio 648 of 10 , to 0.007 for an aspect ratio of 100 , and to 0.0001 for an aspect ratio of 500 . Thus, the presence 649 of a small fraction of ellipsoidal bubbles (e.g., tube pumices) or cracks in a sample could provide an 650 explanation of the permeabilities of volcanic foams whose porosities are below the percolation 651 threshold for mono-disperse spheres.

652 Although percolation theory and the simple power-law relationships between porosity and permeability 653 support the expected relationship between these two foam properties, permeability variations of up to 4 654 orders-of-magnitude at similar porosities clearly indicate that, in addition to porosity, there are other 655 properties of the foams, such as the size distributions of bubbles and pore throats, influencing their 656 permeability, as discussed above.

657 Both crystallinity and bubble anisotropy have been shown to influence the permeability of natural and 
658 experimental magmatic foams, however these influences were not investigated in this study. Degruyter 659 et al. (2010), Schneider et al. (2012), and Burgisser et al. (2017) provide multiple examples of the 660 effects of preferred bubble orientation on magma permeability. The effect of crystals on permeability

661 development in magmatic foams was investigated experimentally in Bai et al. (2011) and Lindoo et al. 662 (2017). Nevertheless, the permeabilities of crystal-rich magmatic samples determined by Saar and 663 Manga (1999), Mueller et al (2008), Bai et al. (2011), Farquharson et al. (2015), Kushnir et al. (2016), 664 and Lindoo et al. (2017) also plot in the same region as the aphyric-to-low-crystallinity samples, but are 665 not shown in Figure 8.

666

667 Comparison of measurements with the models of Degruyter et al. (2010) and Burgisser et al. (2017)

668 The complexity of the relationship between porosity and permeability displayed in Figure 8 has been

669 noted before by many authors, and many models for the calculation of permeability have been

670 constructed and demonstrated to reproduce the results of the individual studies (e.g., Saar and Manga

671 1999; Mueller et al., 2005; Polacci et al., 2008; Bai et al., 2010; Degruyter et al., 2010; Lindoo et al.,

672 2016; Burgisser et al. 2017; LaSpina et al. 2017). Most recently, Burgisser et al. (2017) developed a

673 model for permeability calculations based on modifications and extensions of concepts and equations

674 developed in Degruyter et al. (2010) and successfully applied their new model to multiple data sets.

675 Burgisser et al. (2017) used a modification of the channel-based Carman-Kozeny relationship to model 676 viscous permeability:

677

678

$$
k_{1}=\frac{\Phi_{c}^{n} d_{t}^{2}}{16 \chi \tau^{2}}
$$

679 where $\phi_{c}^{n}$ is the connected porosity raised to the $n$th power, $d_{t}$ is the characteristic diameter of pore 
throats, $\chi$ is the channel circularity:

$$
\chi=\left(\frac{r^{2}}{l^{2}}+\frac{l^{2}}{r^{2}}\right)
$$

682 where $r$ is the equivalent circle radius of the throat and 1 is its major axis $(\chi=2$ for circular pore 683 throats, Degruyter et al. 2010), and $\tau$ is the tortuosity. Degruyter et al. (2010) set $\mathrm{n}=1$ in this equation 684 for their study, but Burgisser et al. (2017) fit their data with this equation and determined a value of $\mathrm{n}=$ 685 2.49. Burgisser et al. (2017) found that this fit reproduced 26 of their 28 viscous permeability 686 measurements to within one log unit.

687 Equation 6 was applied to the samples produced in this study for which the appropriate variables were 688 measured (Table 2) to predict permeabilities using both the value of $n=1$ from Degruyter et al. (2010) 689 and of $n=2.49$ from Burgisser et al. (2017); in both applications the value of $\chi$ was set to 2 (Degruyter 690 et al. 2010). The quality of the model fit to the data was assessed by calculating chi-squared as defined 691 by:

692 chi - squared $=\sum \frac{(\log [\text { calculated permeability }]-\log [\text { measured permeability }])^{2}}{((\log [\text { measured permeability }]))} .(8)$

693 Application of the Degruyter et al. (2010) formulation of Equation 6 with $d_{t}$ equaling the average pore694 throat diameter, $\chi=2$ and $n=1$ predicted the permeabilities of 17 out of 23 permeability 695 determinations to within $1 \log$ unit (all were within $1.6 \log$ units) and produced a chi-squared value of 6961.13 (Fig. 9a). With Burgisser et al.'s (2017) value of $n=2.49,19$ permeability predictions were within $6971 \log$ unit of our determinations (all were within $1.4 \log$ units), and the chi-squared value was 1.14 (Fig. 698 9a). The fit of these models to the measurements of this study is impressive when considering the 699 almost 5 orders of magnitude spread in the lattice-Boltzmann permeabilities; however the trend 700 between predicted and measured permeabilities is at high angles to the slope of the perfect 1:1 701 correlation line between model and measurement (Fig. 9a), indicating the need for further model 
refinement.

703

704 Toward a better model of the viscous permeability of magmatic foams by considering the largest pore

705 throat area

706 We wish to improve upon the models of Degruyter et al. (2010) and Burgisser et al. (2017) using the

707 lattice-Boltzmann permeabilities found in this study. However, there are three caveats that must be

708 considered for the model discussed below. The first is that the model is only calibrated for

709 permeabilities between $\sim 10^{-15}$ and $10^{-10} \mathrm{~m}^{2}$. The second is that we have no technique to determine

710 when an individual sample becomes permeable, although we can approximate the permeability

711 threshold at $\Phi \sim 0.3$ (the percolation threshold for interpenetrating spheres) or use the methods

712 presented in Burgisser et al. (2017) to estimate the permeability threshold if no other information is

713 available. The third caveat is that the model is probably only applicable to isotropic, or nearly

714 isotropic, samples.

715 Fluid conductivity in porous media can be related to a random resistor network by application of

716 percolation theory (Stauffer and Aharony 1994; Blower et al. 2001). Applying this paradigm, the

717 porous network can be envisioned as resistors interconnected to one another that create a continuous

718 circuit when the permeability threshold is exceeded and fluid can flow across the sample. The

719 connections between resistors in the network can be either in parallel or in series (Stauffer and Aharony

720 1994; Blower et al. 2001). The conductivity of the network is related to the specifics of the pore throat

721 cross-sectional area, as expressed in the numerator of the Carman-Kozeny relationship as the square of

722 the characteristic throat diameter, $\mathrm{d}_{\mathrm{c}}{ }^{2}$ (Equation 6). Given a complete description of the lengths and

723 diameters of the pore throats, together with detailed information about their connections in either series

724 or parallel sub-circuits, a complete model of sample permeability should be calculable, but these data 
725 are not available. However, by making the simplifying assumption that the largest pore throat

726 dominates the permeability (as the minimum-valued resistor in a parallel circuit dominates the current

727 flow), the width of that throat, $d_{\max }$, can be used as the characteristic pore throat diameter in Equation 6.

728 Support for the idea of parallel connections between bubbles is provided by the high values of the 729 connective density, ranging from $10^{3}$ to $10^{4} \mathrm{~mm}^{-3}$ (Table 2). Replacing the average throat diameter

730 with the maximum throat diameter, and leaving $\chi=2$, in Equation 6 did not significantly improve the

731 fit of either the Degruyter et al. (2010) or Burgisser et al. (2017) models; however the Burgisser et al.

732 (2017) model yielded calculated permeabilities consistently above the measured ones by a factor of 5.

733 Based upon this observation, an empirical value of $\chi=10$ (or a fitting factor of 5 times the original

734 value of $\chi=2$ ) was chosen, and the resulting fit of the Burgisser et al. model to the data is remarkable

735 (Fig. 9b), with all but one of the measurements reproduced to within one log unit and a chi-squared

736 value of 0.01 . The Degruyter et al. (2010) model also reproduces all but one of the measurements to

737 within $1 \log$ unit, but yields a chi-squared value twice that of the Burgisser et al. model, 0.02.

738 The challenge in applying this model to predict permeability from porosity measurements is that the

739 tortuosity and the maximum throat diameter needed for the calculation typically are not known (cf.

740 Burgisser et al. 2017). In most cases, published studies only provide the average bubble diameter and

741 the porosity. However, the relationship found in this study between tortuosity and porosity (Eqn. 5a)

742 can be used to estimate the tortuosity. Additionally, we found that the maximum throat diameter can

743 be related to the average bubble size, $\mathrm{d}_{\mathrm{avg}}$ bubble $(\mathrm{m})$, by

$$
d_{\max }=2.22251 \times 10^{-5} \ln \left(d_{\mathrm{avg}}^{\text {buble }}\right)+2.69501 \times 10^{-4}
$$

745 To test this model, the permeabilities of samples within the range of our calibration, $10^{-15}$ to $10^{-10} \mathrm{~m}^{2}$,

746 from Bai et al. (2010) and the isotropic pumices of Burgisser et al. (2017) were estimated using the

747 correlations between tortuosity and porosity and between the average bubble size and the maximum 
748 throat diameter (Fig. 9c). We did not apply the model to the non-isotropic samples of Burgisser et al.

749 (2017) because we are unsure whether our correlations would apply to these samples and lack the

750 necessary data to test our model on non-isotropic samples.

751 Although there is clearly a degradation in the accuracy of the model when the correlations are used to

752 estimate the tortuosity and maximum throat diameter rather than their measured values (cf. Fig. 9c with

753 Fig. 9b), the permeability of 11 of the 14 samples from Bai et al. (2010) are reproduced within $1 \log$

754 unit, and the maximum difference between all estimated and measured permeabilities is $1.9 \log$ units.

755 The chi-squared value (Eqn. 8) for all of the Bai et al. (2010) and the model is 0.77. The estimated

756 permeabilities of all 13 isotropic samples from Burgisser et al. (2017) are within 0.9 of a log unit of the

757 measured values, and the chi-squared value is 0.35 . The accuracy of this model is similar to that

758 reported by Burgisser et al. (2017) who found that their fit to Equation 6 could reproduce 26 out of the

75928 (isotropic and anisotropic) samples they investigated to within $1 \log$ unit.

760 This test of the model indicates its utility for estimating the permeability of samples with knowledge of

761 only the porosity and the average bubble diameter. However, as shown in Figure 9c, the model has a

762 tendency to overestimate the permeabilities by a factor of $\sim 5$. An ad hoc correction could be made for

763 this overestimation, but even without such correction the test indicates that permeabilities can be

764 calculated to within an order of magnitude with the model.

765

766 Role of bubble growth rate on permeability

767 Although we were unable to successfully determine permeabilities at the same porosity from

768 experiments with the same composition at different heating rates, our results agree with previous

769 studies indicating that bubble growth rates significantly influence permeabilities (Rust and Cashman

770 2004; Burgisser and Gardner 2004; Mueller al. 2005, 2008; Takeuchi et al. 2009; Castro et al. 2012; 
771 Lindoo et al. 2016). In particular, Lindoo et al. (2016) noted that increasing decompression rates in

772 their experiments, leading to increasing bubble growth rates, resulted in an increasing percolation

773 threshold. This hypothesis is consistent with two observations on the basaltic composition where the

774 slowly heated experiment DRB2012-7f-10 $\left(1^{\circ} \mathrm{C} \mathrm{s}^{-1}\right)$ with a porosity of 0.55 has a permeability $(1.5 \mathrm{x}$

$\left.77510^{-11} \mathrm{~m}^{2}\right)$ similar to the rapidly heated $\left(6^{\circ} \mathrm{C} \mathrm{s}^{-1}\right), 0.73$ porosity experiment DRB2012-7c-f $\left(2.9 \times 10^{-11}\right.$

$776 \mathrm{~m}^{2}$ ), whereas the fit from Bai et al. (2010) predicts a permeability of at least $5 \times 10^{-11} \mathrm{~m}^{2}$ at a porosity

777 of 0.73 .

778 We also note that there may be a correlation between the bubble growth rate and the size distribution of

779 pore throats that significantly influences permeability. We propose this tentative hypothesis because of

780 the often-lower permeabilities of the rapidly heated $\left(5^{\circ} \mathrm{C} \mathrm{min}^{-1}\right)$ andesitic foam in comparison to the

781 more slowly heated $\left(\sim{ }^{\circ} \mathrm{C} \mathrm{min}^{-1}\right)$ trachyandesitic and dacitic foams with similar porosities (Table 2,

782 Figure 7).

783 Despite the need for further experiments to quantitatively constrain the effects of decompression and

784 growth rates on the permeability of silicate foams, we suggest that the orders-of-magnitude variability

785 seen in permeability at similar porosities in Figure 8 is significantly controlled by the bubble growth

786 rate. The formation of a pore throat between two bubbles requires them to partially coalesce; for

787 coalescence to occur the interbubble melt film (IBF) must thin to the point where it fails, estimated to

788 be a thickness of $0.5 \mu \mathrm{m}$ in a rhyolitic melt by Castro et al. (2012). The rate at which the IBF thins is a

789 function of the surface tension and bubble size (Equation 4), and the timescales of thinning vary from

790 less than a second (basaltic melts) to thousands of seconds (dacitic melts), based upon the analyses of

791 Castro et al. (2012) and Nguyen et al. (2013). In the case where bubbles are growing on a timescale

792 shorter than that of IBF thinning, coalescence is not as effective in creating large pore throats as at

793 slower growth rates, and permeabilities at equal porosities are lower than in the case where bubble 
794 growth is slower than IBF thinning. Consideration of the lubrication and drag forces during bubble

795 growth suggests the possibility of a bubble-size-dependence of connectivity (and therefore

796 permeability). These considerations will be explored in future studies.

797 Although we think that the rate of gas exsolution plays a significant role in creating the wide spread of

798 permeability at equivalent porosities, we recognize that other factors may affect the observed variations

799 in permeability, such as the effects of bubble orientation (e.g., Degruyter et al. 2010; Burgisser et al.

800 2017) and the presence of crystals in the magma (e.g., Bai et al. 2011; Lindoo et al. 2017).

801

802 Conclusions

803 A complete characterization of magmatic foams is required to model permeability because

804 permeability-porosity relationships alone do not provide sufficient data for accurate modeling and

805 prediction. Furthermore, average properties of the foam, in particular average pore-throat diameters,

806 appear to be insufficient to fully characterize permeability. Complete measurements of porosity,

807 bubble and pore throat size distributions, as well as tortuosity, are required to model accurately the

808 permeability of magmatic foams. In particular, we stress the apparent importance of the largest pore

809 throat on the permeability of magmatic foams. We propose one such model, which we consider a step

810 in the right direction, that can be used to benchmark future studies. The results of this study are

811 consistent with previous work indicating the importance of the bubble growth rate on the permeability

812 of magmatic foams. Higher growth rates appear to produce lower permeabilities, and the effect of

813 growth rate on permeability may explain a significant portion of the orders-of-magnitude spread in

814 permeabilities at similar porosity.

815 


\section{Acknowledgements}

817 D.R. Baker thanks NSERC for their continued support for his research through the Discovery Grant

818 Program. All of the members of the TOMCAT team and of the Swiss Light Source at the Paul Scherrer

819 Institut are thanked for their creation of the facility that allowed this study to be done and their

820 continuing dedication to providing support to external users of the beamline.

821 We also thank the editor, K. Cashman and the reviewers H. Wright, L. Chevalier and A. Burgisser for

822 their detailed and thoughtful comments that significantly improved the presentation of our research in

823 this contribution.

\section{References}

825 Al-Raoush RI, Madhoun IT (2017) TORT3D: A MATLAB code to compute geometric tortuosity from 826 3D images of unconsolidated porous media, Powder Tech 320: 99-107

827 Archie GE (1942) The electrical resistivity log as an aid in determining some reservoir characteristics.

$828 \quad$ Trans Am Inst Mineral Meteorol 146:54-62.

829 Bai L, Baker DR, Hill RJ (2010) Permeability of vesicular Stromboli basaltic glass: Lattice Boltzmann $830 \quad$ simulations and laboratory measurements. J Geophys Res 115:B07201.

831 doi:10.1029/2009JB007047

832 Bai L, Baker DR, Polacci M, Hill RJ (2011) In-situ degassing study on crystal-bearing Stromboli

833 basaltic magmas: Implications for Stromboli Eruptions. Geophys Res Lett 38: L17309

834 Baker DR (2004) Piston-cylinder calibration at 400 to $500 \mathrm{MPa}$ : a comparison of using water solubility 835 in albite melt and $\mathrm{NaCl}$ melting. Am Mineral 89:1553-1556

836 Baker DR Eggler DH (1987) Compositions of anhydrous and hydrous melts coexisting with plagioclase, 837 augite, and olivine or low-Ca pyroxene from 1 atm. to 8 kbar: application to the Aleutian volcanic 
839 Baker DR, Polacci MR, LaRue A (2011) A study on the reproducibility of counting vesicles in volcanic rocks. Geosphere, doi: 10.1130/GES00553.1

841 Baker DR, Brun F, O’Shaughnessy C, Mancini L, Fife JL, Rivers M (2012) A four-dimensional X-ray 842 tomographic microscopy study of bubble growth in basaltic foam. Nat Comm 3: 1135. doi: $10.1038 /$ ncomms 2134

844 Blower JD (2001) Factors controlling permeability-porosity relationships in magmas. Bull Volcanol 63:497-504

846 Bouvet de Maisonneuve C, Bachmann O, Burgisser A (2008) Characterization of juvenile pyroclasts from 847 the Kos Plateau Tuff (Aegean Arc): insights into the eruptive dynamics of a large rhyolitic eruption. Bull Volcanol doi: 10.1007/s00445-008-0250-x

Browne B, Szramek L (2015) Chapter 9: Rates of magma ascent and storage. The Encyclopedia of

851 Brun F, Dreossi D (2010) Efficient curve-skeleton computation for the analysis of biomedical 3D 852 images. Biomed Sci Instrum 46:475-480

853 Brun F, Mancini L, Kasae P, Favretto S, Dreossi D, Tromba G (2010) Pore3D: A software library for 854 quantitative analysis of porous media. Nucl Instr Methods Phys Res A 615:326-332

855 Burgisser A, Gardner JE (2004) Experimental constraints on degassing and permeability in volcanic 856 conduit flow. Bull Volcanol 67:42-56. http://dx.doi.org/10.1007/s00445-004-0359-5

857 Burgisser A, Chevalier L, Gardner JE, Castro JM (2017) The percolation threshold and permeability evolution of ascending magmas. Earth Planet Sci Lett 470:37-47

859 Carman PC (1937) Fluid flow through a granular bed. Trans Inst Chem Eng London, 15:150-156 
860 Castro JM, Burgisser A, Schipper CI, Mancini S (2012) Mechanisms of bubble coalescence in silicic

$861 \quad$ magmas. Bull Volcanol 74:2339-2352

862 Colombier M, Wadsworth FB, Gurioli L, Scheu B, Kueppers U, Di Muro A, Dingwell DB (2017) The 863 evolution of pore connectivity in volcanic rocks. Earth Planet Sci Lett 462:99-109

864 Consiglio R, Baker DR, Paul G, Stanley HE (2003) Continuum percolation thresholds for mixtures of $865 \quad$ spheres of different sizes. Physica A 319:49-55

866 Cox SJ, Graner F (2004) Three-dimensional bubble clusters: Shape, packing, and growth rate. Phys 867 Rev E 69: 031409

868

869

870 $72: 63-74$

871

Domb C (1972) A note on the series expansion method for clustering problems. Biometrika 59:209-211

872

873

874

Dowd B, Campbell GH, Marr RB, Nagarkar V, Tipnis S, Axe L, Siddons DP (1999) Developments in synchrotron x-ray computed tomography at the National Synchrotron Light Source. Developments in X-Ray Tomography II, Proc SPIE 3772:224-236

875 Fagents SA, Gregg TKP, Lopes RMC (2013) Modeling Volcanic Processes: The Physics and 876 Mathematics of Volcanism. Cambridge University Press, Cambridge, $421 \mathrm{p}$

877 Farquharson J, Heap MJ, Varley NR, Baud P, Reushclé T (2015) Permeability and porosity 878 879 relationships of edifice-forming andesites: A combined field and laboratory study. Jour Volcan Geotherm Res 297:52-68

880 Ferguson DJ, Gonnermann HM, Ruprech P, Plank T, Hauri EH, Houghton BF, Swanson DA (2016) 881 Magma decompression rates during explosive eruptions of Kilauea volcano, Hawaii, recorded by 
883 Fife JL, Rappaz M, Pistone M, Celcer T, Mikuljan G, Stampanoni M (2012) Development of a laser884 based heating system for in-situ synchrotron-based x-ray tomographic microscopy, J. Synchrotron Radiation 19:352-358

886 Fortin M-A, Riddle J, Desjardins-Langlais Y, Baker DR (2015) The effect of water on the sulfur concentration at sulfide saturation (SCSS) in natural melts. Geochim Cosmochim Acta 160:100-

Gaonac'h H, Lovejoy S, Stix J, Scherzter D (1996) A scaling growth model for bubbles in basaltic lava flows. Earth Planet Sci Lett 139:395-409.

891 Garboczi EJ, Snyder KA, Douglas JF, Thorpe MF (1995) Geometrical percolation threshold of overlapping ellipsoids. Physical review E 52:819-828

893 Giordano D, Nichols ARL, Dingwell DB (2005) Glass transition temperatures of natural hydrous 894 melts: a relationship with shear viscosity and implications for the welding process. J Volcan $895 \quad$ Geotherm Res 142: 105-118

896 Giordano D, Russell JK, Dingwell DB (2008) Viscosity of magmatic liquids: A model. Earth Planet 897 Sci Lett 278:123-134

898 Hildebrand T, Rüegsegger P (1997) A new method for the model-independent assessment of thickness 899 in three-dimensional images. J Microscopy 185:67-75

900 Hill RJ, Koch DL,Ladd AJC (2001) The first effects of fluid inertia on flows in ordered and random $901 \quad$ arrays of spheres. J Fluid Mech 448:213-241. doi:Ziff10.1017/S0022112001005948

902 Hill RJ, Koch DL (2002) The transition from steady to weakly turbulent flow in a close-packed ordered 903 array of spheres. J Fluid Mech 465:59-97. doi:10.1017/S0022112002008947 
904 Humphreys MCS, Menand T, Blundy JD, Klimm K (2008) Magma ascent rates in explosive eruptions:

905 Constraints from $\mathrm{H}_{2} \mathrm{O}$ diffusion in melt inclusions. Earth Planet Sci Lett 270:25-40

906 Klug C, Cashman KV (1996) Permeability development in vesiculating magmas: implications for 907 fragmentation. Bull Volcan 58:87-100

908 Kraynik AM, Reinelt DA, van Swol F (2004) Structure of random foam. Phys Rev Lett 93: 208301, $909 \quad$ 10.1103/PhysRevLett.93.208301

910 Kushnir ARL, Martel C, Bourdier J-L, Heap MJ, Reushclé T, Erdmann S, Komorowski J-C, Cholik N

911 (2016) Probing permeability and microstructure: Unravelling the role of a low-permeability 912 dome on the explosivity of Merapi (Indonesia). Jour Volcan Geotherm Res 316:56-71

913 LaRue A (2012) Bubble size distributions and magma-water interaction at Eyjafjallajökull volcano, 914 Iceland. M.Sc. Thesis, McGill University

915 La Spina G, Polacci M, Burton M, de’ Michieli Vitturi M (2017) Numerical investigation of 916 permeability models for low viscosity magmas: Application to the 2007 Stromboli effusive 917 eruption. Earth Planet Sci Lett 473:279-290. https://doi.org/10.1016/j.eps1.2017.06.013

918 Lindoo A, Larsen JF, Cashman KV, Dunn AL, Neill OK (2016) An experimental study of permeability 919 development as a function of crystal-free melt viscosity. Earth Planet Sci Lett 435:45-54

920 Lindoo A, Larsen JF, Cashman KV, Oppenheimer J (2017) Crystal controls on permeability

921 development and degassing in basaltic andesite magma. Geology 45: 831-834

922 Lindquist WB (2002) Quantitative analysis of three dimensional X-ray tomographic images. Proc.

923 SPIE 4503:103-115

924 Lindquist WB, Lee SM (1996) Medial axis analysis of void structure in three-dimensional tomographic 925 images of porous media. J. Geophys Res 101:8297-8310 
926 Liu Y, Samaha N-T, Baker DR (2007) Sulfur concentration at sulfide saturation (SCSS) in magmatic 927 silicate melts. Geochim Cosmochim Acta 71:1783-1799

928 Liu Y, Zhang Y, Behrens $\mathrm{H}$ (2005) Solubility of $\mathrm{H}_{2} \mathrm{O}$ in rhyolitic melts at low pressures and a new 929 empirical model for mixed $\mathrm{H}_{2} \mathrm{O}-\mathrm{CO}_{2}$ solubility in rhyolitic melts. J Volcan Geotherm Res $930 \quad 143: 219-235$

931 Lorenz CD, Ziff RM (2001) Precise determination of the critical percolation threshold for the three932 dimensional 'Swiss cheese' model using a growth algorithm. J Chem Phys 114:3659-3661

933 Marone F, Stampanoni M (2012) Regridding reconstruction algorithm for real time tomographic 934 imaging. J Synchrotron Rad 19:1029-1037

935 Mokso R, Marone F, Stampanoni M (2010) Real time tomography at the Swiss Light Source. AIP Conf $936 \quad$ Proc $1234: 87-90$

937 Mokso R, Schlepuetz CM, Theidel G, Billich H, Schmid E, Celcer T, Mikuljan G, Sala L, Marone F, 938 Schlumpf N, Stampanoni M (2017) GigaFRoST: The Gigabit Fast Readout System for 939 Tomography. Jour Synchrotron Rad doi.org/10.1107/S1600577517013522

940 Mueller S, Melnik O, Spieler O, Scheu B, Dingwell DB (2005) Permeability and degassing of dome 941 lavas undergoing rapid decompression: An experimental determination. Bull Volcan 67:526-538

942 Mueller S, Scheu B, Spieler O, Dingwell DB (2008) Permeability control on magma fragmentation. $943 \quad$ Geology 36:499-402

944 Navon O, Lyakhovsky, V, (1998) Vesiculation processes in silicic magmas. In: Gilbert, J.S., Sparks, 945 R.S.J. (Eds.), The Physics of Explosive Volcanic Eruptions. Geol Soc Spec Pub Lond 145: 2794650.

947 Neuville DR, Courtial P, Dingwell DB, Richet P (1993) Thermodynamic and rheological properties of 
949 Newman MEJ (2005) Power laws, Pareto distributions and Zipt's law. Contemporary Physics 46:323$950 \quad 351$

951 Nguyen CT, Gonnermann HM, Chen Y, Huber C, Maiorano AA, Gouldstone A, Dufek J (2013) Film 952 drainage and the lifetime of bubbles. Geochem Geophys Geosys 14: 10.1002/ggge.20198

953 Ni H, Zhang L (2018) A general model of water diffusivity in calcalkaline silicate melts and glasses. $954 \quad$ Chem Geol 478:60-68.

955 Odgaard A, Gundersen HJG (1993) Quantification of connectivity in cancellous bone, with special 956 emphasis on 3-D reconstructions. Bone 14:173-182

957 Ogata R, Odagaki T, Okazaki K (2005) Effects of poly-dispersity on continuum percolation. J Phs $958 \quad$ Condens Matter 17:4531-4538

959 Papale P, Moretti R, Barbato D (2006) The compositional dependence of the saturation surface of $\mathrm{H}_{2} \mathrm{O}$ $960+\mathrm{CO}_{2}$ fluids in silicate melts. Chem Geol 229:78-95

961 Polacci M, Baker DR, Bai L, Mancini L (2008) Large vesicles record pathways of degassing at basaltic 962 volcanoes. Bull Volcanol 70:1023-1029. https://doi.org/10.1007/s00445-007-0184-8

963 Polacci M, Baker DR, La Rue A, Mancini L, Allard P (2012) Degassing behaviour of vesiculated 964 basaltic magmas: an example from Ambrym volcano, Vanuatu Arc. Jour Volcan Geotherm Res $965 \quad 233-234: 55-64$.

966 Polacci M, Bouvet de Maisonneuve C, Giordano D, Piochi M, Mancini L, Degruyter W, Bachmann O 967 (2014) Permeability measurements of Campi Flegrei pyroclastic products: An example from the 968 Campanian Ignimbrite and Monte Nuovo eruptions. Jour Volcan Geotherm Res 2272:16-22.

969 Rivers ML, Wang Y (2006) Recent developments in microtomography at GeoSoilEnviroCARS. 
971 Rust AC, Cashman KV (2004) Permeability of vesicular silicic magma: Inertial and hysteresis effects. $972 \quad$ Earth Planet Sci Lett 228:93-107

973 Rust AC, Cashman KV (2011) Permeability controls on expansion and size distributions of pyroclasts. J Geophys Res 116:B11202 26:111-114

Schneider A, Rempel AW, Cashman KV. (2012) Conduit degassing and thermal controls on eruption styles at Mount St. Helens. Earth Planet Sci Lett 357-358: 347-354

Shaw HR (1972) Viscosities of magmatic silicate liquids: an empirical method of prediction. Am J Sci 272:870-893

981

982

983

984

985

986

987

988

989

990

991

Sparks RSJ (2003) Dynamics of magma degassing. In: Oppenheimer, C., Pyle, D.M., and Barclay, J. (eds) Volcanic Degassing. Geological Society, London, Special Publications 213:5-22

Spieler O, Kennedy B, Kueppers U, Dingwell DB, Scheu B, Taddeucci J (2004) The fragmentation threshold of pyroclastic rocks. Earth Planet Sci Lett 226:139-148

Stauffer D, Aharony A. (1994) Introduction to Percolation Theory, $2^{\text {nd }}$ Edition. Taylor \& Francis, London, $181 \mathrm{p}$

Takeuchi S, Nakashima S, Tomiya A (2008) Permeability measurements of natural and experimental volcanic materials with a simple permeameter: Toward and understanding of magmatic degassing processes. J Volcan Geotherm Res 177:329-339.

Takeuchi S, Tomiya A, Shinohara H (2009) Degassing conditions for permeable silicic magmas: implications from decompression experiments with constant rates. Earth Planet Sci Lett 283:101- 
993 Thovert J.F., Salles J., Adler P.M. (1993) Computerized characterization of the geometry of real porous 994 media: their discretization, analysis and interpretation. J. Microscopy 170:65-79

995 Tomasi C, Manduchi R (1998) Bilateral filtering for gray and color images. Sixth Internat Conf Comp 996 Vision: 839-846

997 Wright HMN, Cashman KV, Gottesfeld EH, Roberts JJ (2009) Pore structure of volcanic clasts:

998 Measurements of permeability and electrical conductivity. Earth Planet Sci Lett 280:93-104

999 Yokoyama T, Takeuchi S (2009) Porosimetry of vesicular volcanic products by a water-expulsion 1000 method and the relationship of pore characteristics to permeability. J Geophys Res 114:B0221. 1001 doi:10.1029/2008JB005758

1002 Zandomeneghi D, Voltolini M, Mancini L, Brun F, Dreossi D, Polacci M (2010) Quantitative analysis 1003 of X-ray microtomography images of geomaterials: Application to volcanic rocks. Geosphere $1004 \quad 6: 793-804$

1005 Zhou J-Q, Chen Y-F, Wang L, Rayani Cardenas M. (2019) Universal relationship between viscous and 1006 inertial permeability of geologica porous media. Geophys Res Lett 46:1441-1448.

1007 Ziff RM, Torquato S (2017) Percolation of disorderd jammed sphere packings. J Phys A Math Theor 1008 50: 085001, doi:10.1088/1751-8121/aa5664

1009

1010 Figure Captions

1011 Figure 1 a) Slice of complete sample DRB2012_6e_10. The $256 \times 256$ pixel region in this slice 1012 sampled for analysis is shown by the dashed lines. b) Detailed image of the portion of the $256 \times$ 1013256 slice sampled from the larger image. c) Thresholded image of (b) with a smaller $160 \times 160$ 
1014 pixel region used for quantitative measurements shown enclosed by the solid lines. d)

1015 Reconstruction for DRB2012-2a-19, with the one-voxel-wide-skeleton in yellow and the nodes

1016 (intersections of the skeleton branches) in red and orange, demonstrating the effect of only

1017 considering larger interconnections between bubbles by merging voxels together and taking their

1018 average. e) The same reconstruction also considering smaller interconnections based upon the

1019 finest voxel resolution. In this work the choice was made to count both large and small

1020 interconnections of the skeleton and maximize the number of possible skeleton nodes (or bubble

1021 centers) and pore throats (the skeleton connecting the nodes) measured. However this procedure

1022 can over-count the number of nodes; to reduce the number of over-counted nodes, spheres are

1023 centered at each node and isotropically inflated by differing degrees. f) This image demonstrates

1024 the effect of less inflation, producing a higher bubble count, and g) demonstrates the effect of

1025 more inflation resulting in the merger of more spheres and a smaller number of counted bubbles.

1026 The merger is best seen in the small sphere on the left-hand side of the big bubble that is not

1027 merged in (f) and would be counted as a separate bubble, but is merged in (g) and becomes part

1028 of the large bubble. h) The size of the bubble to be counted is determined by a maximal inscribed

1029 sphere that is centered on the center of mass determined from the merged bubbles. Please see

1030 text for further discussion. (Color on line)

1031 Figure 2 a, b, c) Selected 3D renderings of andesitic experiment DRB2012-2a during

1032 vesiculation. In the earliest image the sample is approximately $1 \times 1 \times 2 \mathrm{~mm}$ in size. Due to the

1033 perspective projections of these renderings the scale bars are only approximate. Representative

1034 interior sections of these samples were chosen for quantitative analysis. d, e, f) Corresponding

1035 thresholded tomographic slices (axial slice number 128 near the center of the reconstruction)

1036 from each of the renderings shown in panels $\mathrm{a}, \mathrm{b}$ and $\mathrm{c}$, in which black is the melt and white is 
either the bubbles in the samples or the air around samples (seen in panels e and f). The $500 \mu \mathrm{m}$ scale bar in panel d also applies to panels e and f. Melt viscosities are calculated using Giordano et al. (2008) with 5 wt. $\% \mathrm{H}_{2} \mathrm{O}$ in the melt at $900{ }^{\circ} \mathrm{C}$ and an estimated 1 wt. $\% \mathrm{H}_{2} \mathrm{O}$ at $994{ }^{\circ} \mathrm{C}$; at $1089{ }^{\circ} \mathrm{C}$, near the end of bubble growth, the melt is assumed to be anhydrous for the viscosity calculation. Quantitative measurements of the experiment are provided in Table 2 and Supplemental Data Table 1. Please see Supplemental data for a movie of this sample during 1044 Figure $3 \quad$ a) Bubble size distributions in basaltic sample DRB2012-7. The figure contains 1045 subpanels with bubble size distributions from different experiments with porosities from 0.50 to 1046 0.73. All panels in this and subsequent plots of bubble and pore-throat size distributions are 1047 plotted in order of increasing experimental duration and temperature. Note that in this figure the 1048 sample with a porosity 0.50 was made by reheating the sample with 0.52 porosity (please see 1049 Table 2 and the text for further discussion). In each subpanel a volume-normalized histogram of 1050 the sizes of the bubbles (the bar graphs) is presented together with the cumulative distribution of 1051 the bubble sizes (solid black line). The porosity is given in the upper right corner of each 1052 1053 1054 1055 subpanel. The mean bubble diameter, $\mathrm{d}$, and one-standard deviation about the mean is given in each subpanel. Bin sizes are $5 \mu \mathrm{m}$. b) Corresponding pore throat size distributions in basaltic sample DRB2012-7. The information on the pore-throat distributions is presented in the same manner as the bubble size distributions in Part a. Bin sizes are $5 \mu \mathrm{m}$.

1056 Figure 4 Bubble and pore-throat distributions for andesitic experiment DRB2012-2a. a) Bubble 1057 size distributions for porosities between 0.17 and 0.33 . Bin sizes are $5 \mu \mathrm{m}$. b) Bubble size 1058 distributions for porosities between 0.41 and 0.59 . Bin sizes are $5 \mu \mathrm{m}$. c) Bubble size 1059 distributions for porosities between 0.71 and 0.80 . Bin sizes are $5 \mu \mathrm{m}$. d) Pore throat size 

distributions for porosities between 0.17 and 0.33 . Bin sizes are $5 \mu \mathrm{m}$. e) Pore throat size 1061 distributions for porosities between 0.41 and 0.59 . Bin sizes are $5 \mu \mathrm{m}$. f) Pore throat size 1062 distributions for porosities between 0.71 and 0.80 . Bin sizes are $5 \mu \mathrm{m}$. Please see caption to $1063 \quad$ Figure 3 for a complete description of figure.

1064 Figure 5 Bubble and pore throat distributions for trachyandesitic experiment EFJ-8a. a) Bubble 1065 size distributions for porosities between 0.09 and 0.64 . Bin sizes are $5 \mu \mathrm{m}$. b) Corresponding 1066 pore throat distributions. Bin sizes are $5 \mu \mathrm{m}$. Please see caption to Figure 3 for a complete 1067 description of figure.

1068 Figure 6 Bubble and pore throat distributions for dacitic experiment DRB2012-6e. a) Bubble size 1069 distributions for porosities between 0.38 and 0.84 . Bin sizes are $5 \mu \mathrm{m}$. b) Corresponding pore $1070 \quad$ throat distributions. Bin sizes are $5 \mu \mathrm{m}$. Please see caption to Figure 3 for a complete description 1071 of figure.

1072 Figure 7 Experimental foam permeabilities determined by lattice-Boltzmann simulations plotted 1073 as a function of porosity. Uncertainties in the measured porosity and in the permeability are 1074 estimated at 20 relative percent and typically the same size as, or smaller than, the symbols. The 1075 solid blue line is the power-law fit to the lattice-Boltzmann permeabilities of the andesitic 1076 composition. The proposed relationships between porosity and permeability for basaltic and for 1077 silicic compositions from Bai et al. (2010) are also plotted. (Color on line) Please see text for 1078 further discussion.

1079 Figure 8 Permeability measurements as a function of porosity for aphyric-to-low-crystallinity 1080 vesiculated samples of basaltic to rhyolitic composition from the literature and this study. Three 1081 fits to the data are presented. Fitting all the data with a power law yields the relationship $k_{1}=$ $6.0 \times 10^{-12} \Phi^{4.0}$. A lower bound is constrained by fitting the permeability measurements below 
1087 Figure $9 \quad$ a) Application of the models for the prediction of permeability using the Carman-

1088 Kozeny equations of Degruyter et al. (2010) and of Burgisser et al. (2017). The line labelled 1:1

1089 represents a perfect fit of a model to the data; the line labeled " $5 x$ " represents the modeled values 1090 multiplied by 5 and the line labeled " $0.2 x$ " represents the modeled values multiplied by 0.2 . In 1091 these models the average pore throat value (Table 2) was used as the characteristic diameter of 1092 pore throats. b) Modification of the models of Degruyter et al. and of Burgisser et al. by using 1093 the maximum pore throat as the characteristic diameter and an empirical value of $\chi=10$. Note 1094 the excellent fit of the Burgisser et al. model to the measurements. c) Comparison of the 1095 modified Burgisser et al. model and measured permeabilities of samples from Bai et al. (2010) 1096 and of the isotropic samples from Burgisser et al. (2017) using relationships between the average 1097 bubble diameter and the largest throat diameter and between the porosity and the tortuosity, as 1098 determined in this study. The data from Bai et al. (2010) and from Burgisser et al. (2017) were 1099 not used to calibrate the model. Please see text for further discussion. (Color on line)

1101 Table 1

1102 Table 2

1103 Supplementary Movie

1104 Supplementary Data Table

1105 experiments.
Starting glass compositions

Experimental results

Bubble growth in DRB2012-2a

Measured diameters of bubbles and pore throats in the 
1106 Supplementary Figure 1 1107 online).
Connectivity versus porosity in the experimental samples (in color 
ف
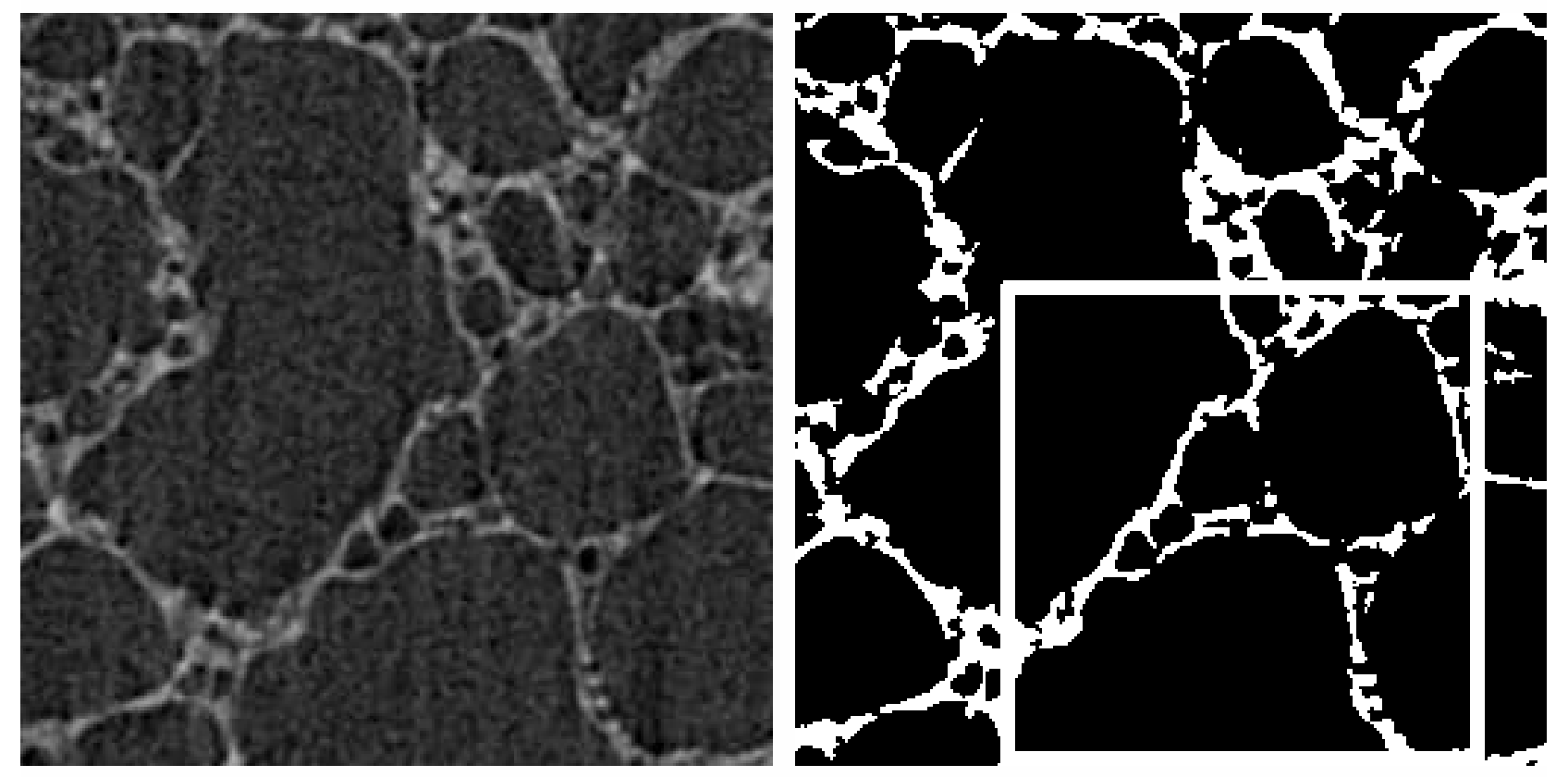

ఠ

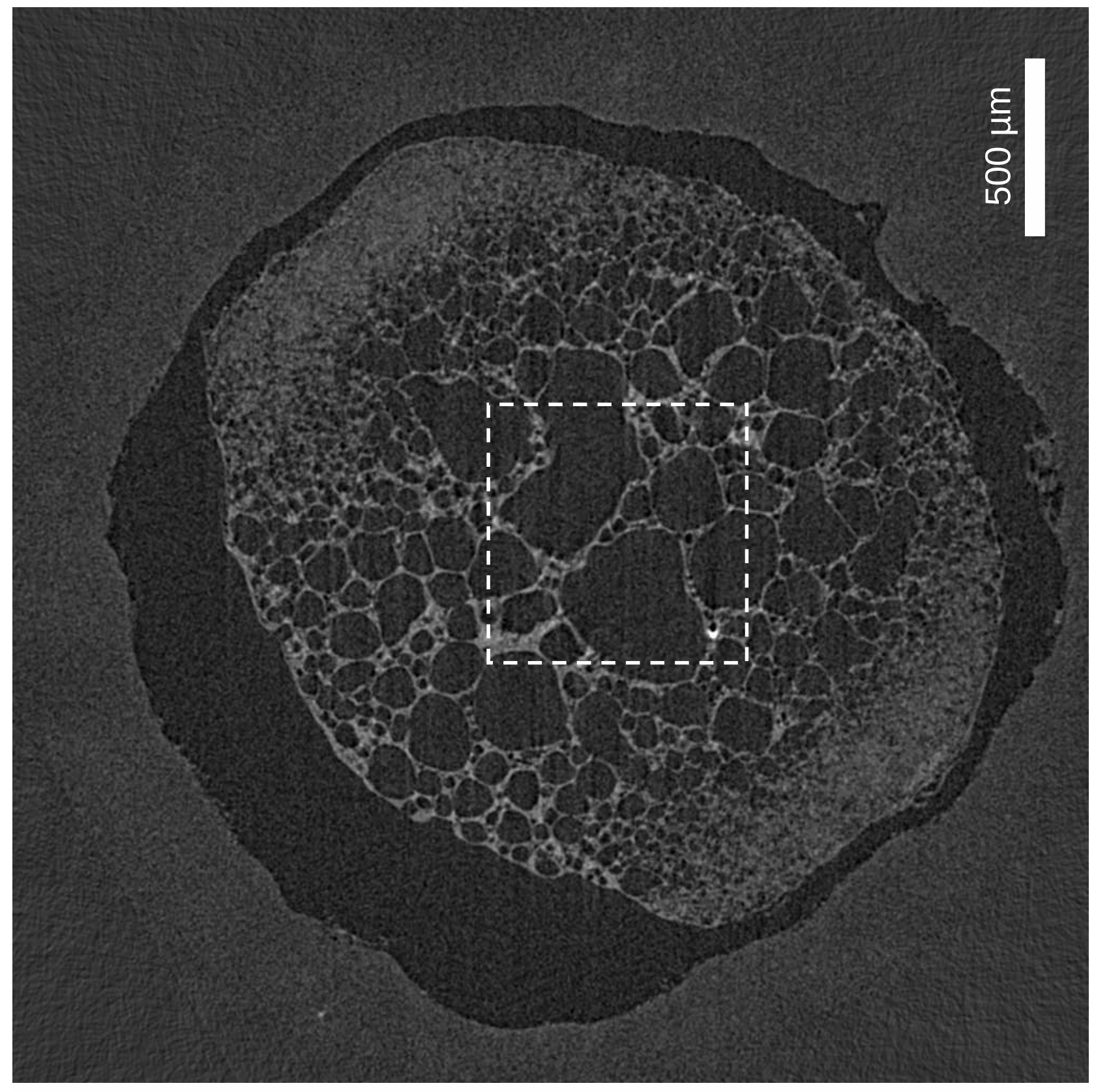



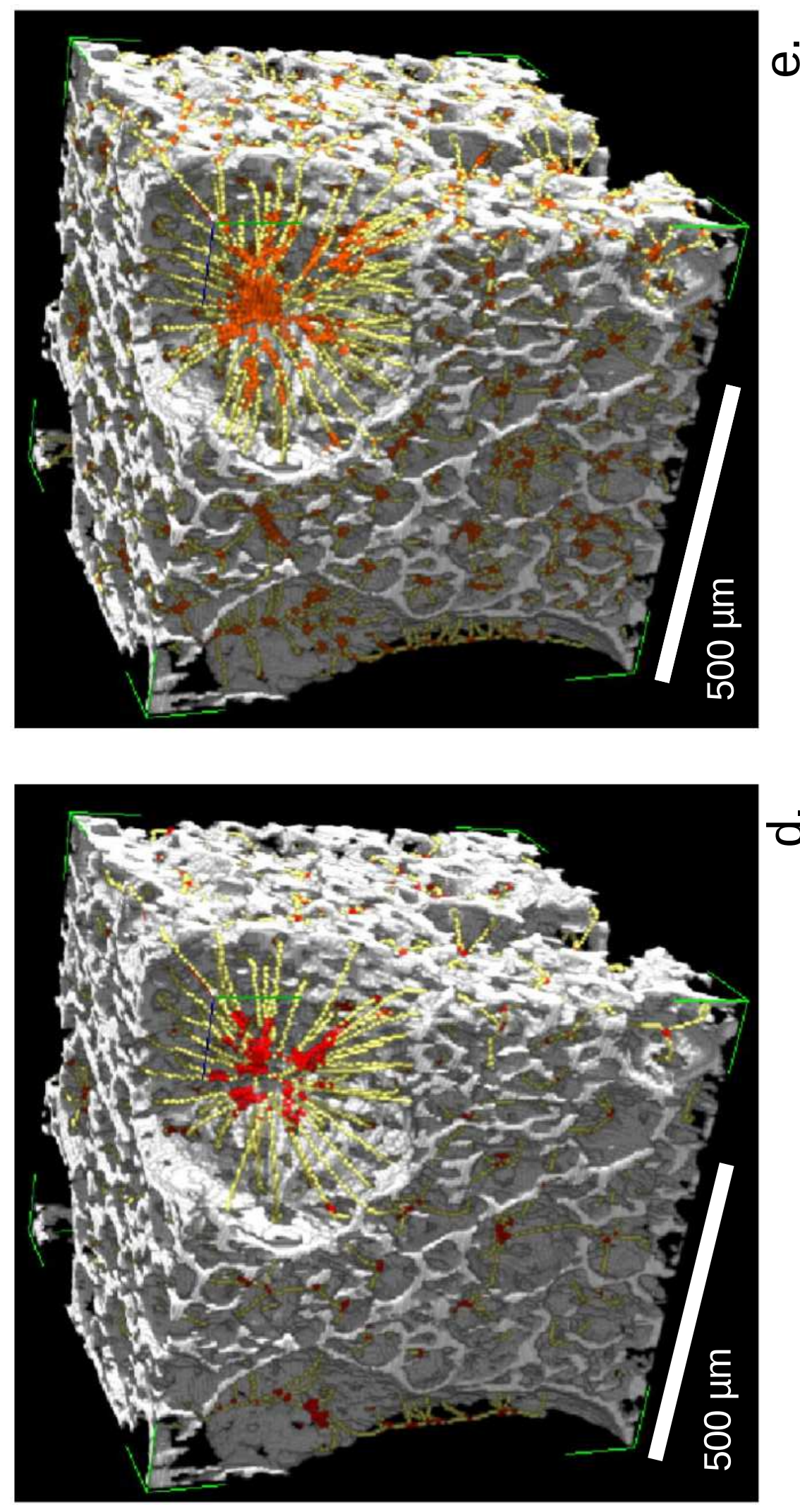

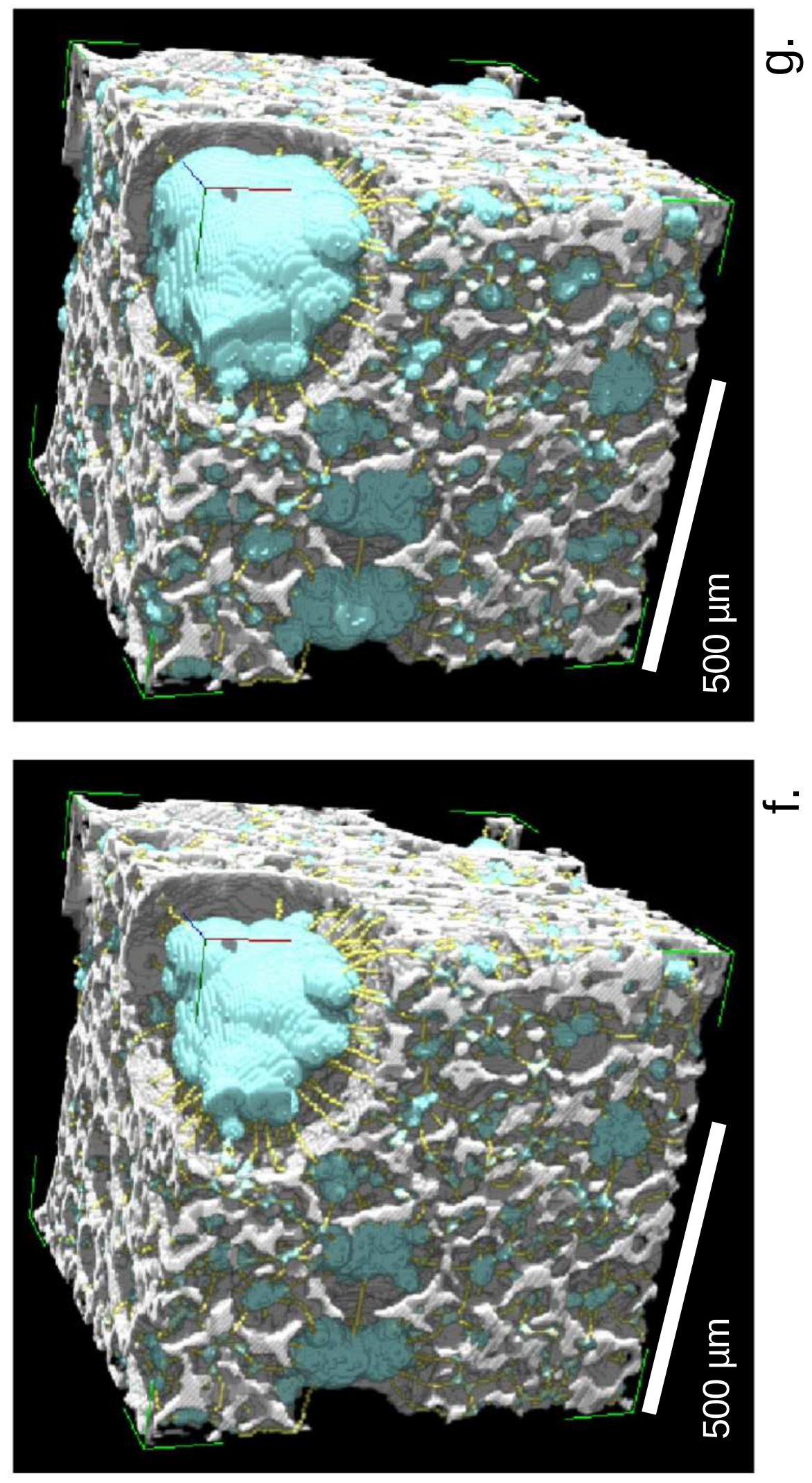


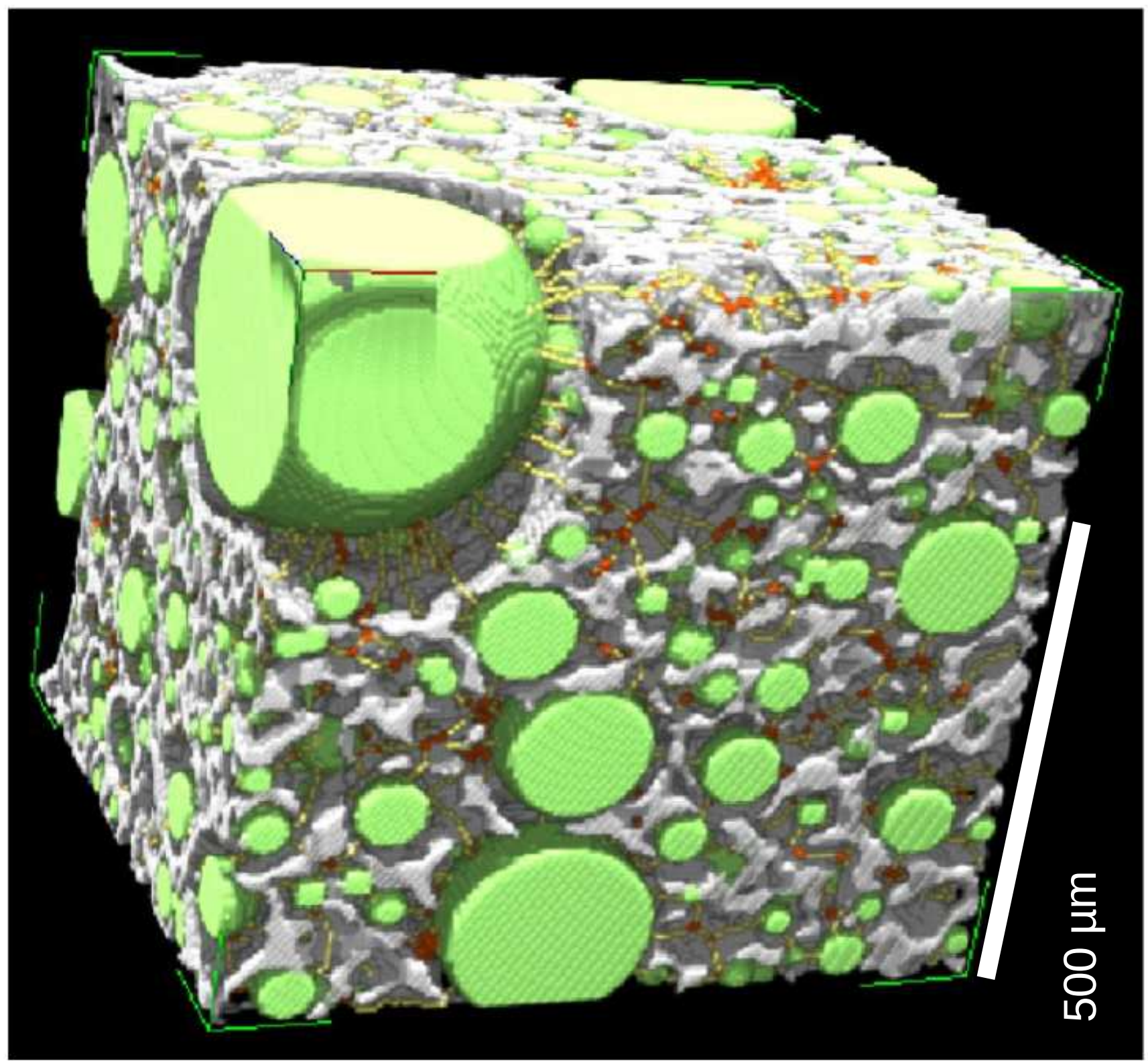

드 


$$
\begin{gathered}
900{ }^{\circ} \mathrm{C}, \text { viscosity } \sim 7.5 \times 10^{2} \mathrm{~Pa} \mathrm{~s}, \Phi=0.17 \\
\left(60 \mathrm{~s} \text { duration above } 600{ }^{\circ} \mathrm{C}\right)
\end{gathered}
$$
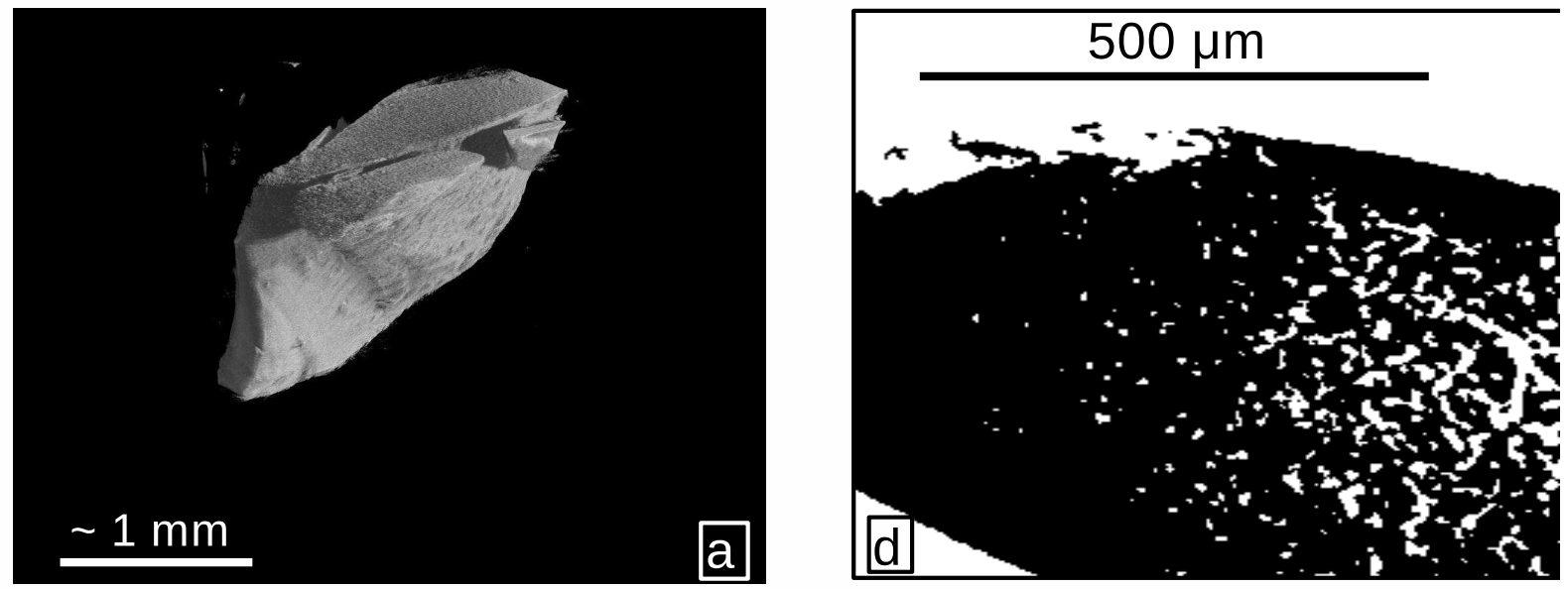

$994{ }^{\circ} \mathrm{C}$ viscosity $\sim 3.1 \times 10^{3} \mathrm{~Pa} \mathrm{~s}, \quad \Phi=0.28$ (79 s duration above $600^{\circ} \mathrm{C}$ )
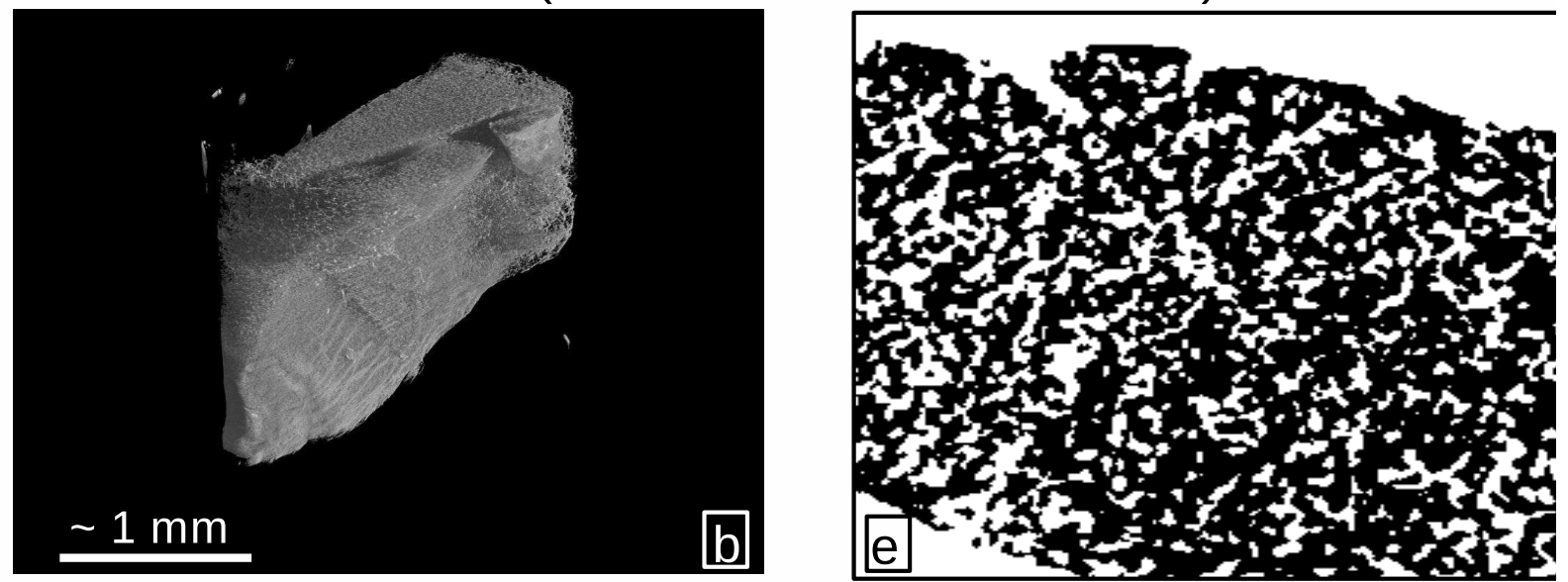

$1089^{\circ} \mathrm{C}$ Viscosity

$6.7 \times 10^{3} \mathrm{~Pa} \mathrm{~S}, \Phi=0.79$ (98 s duration above $600^{\circ} \mathrm{C}$ )
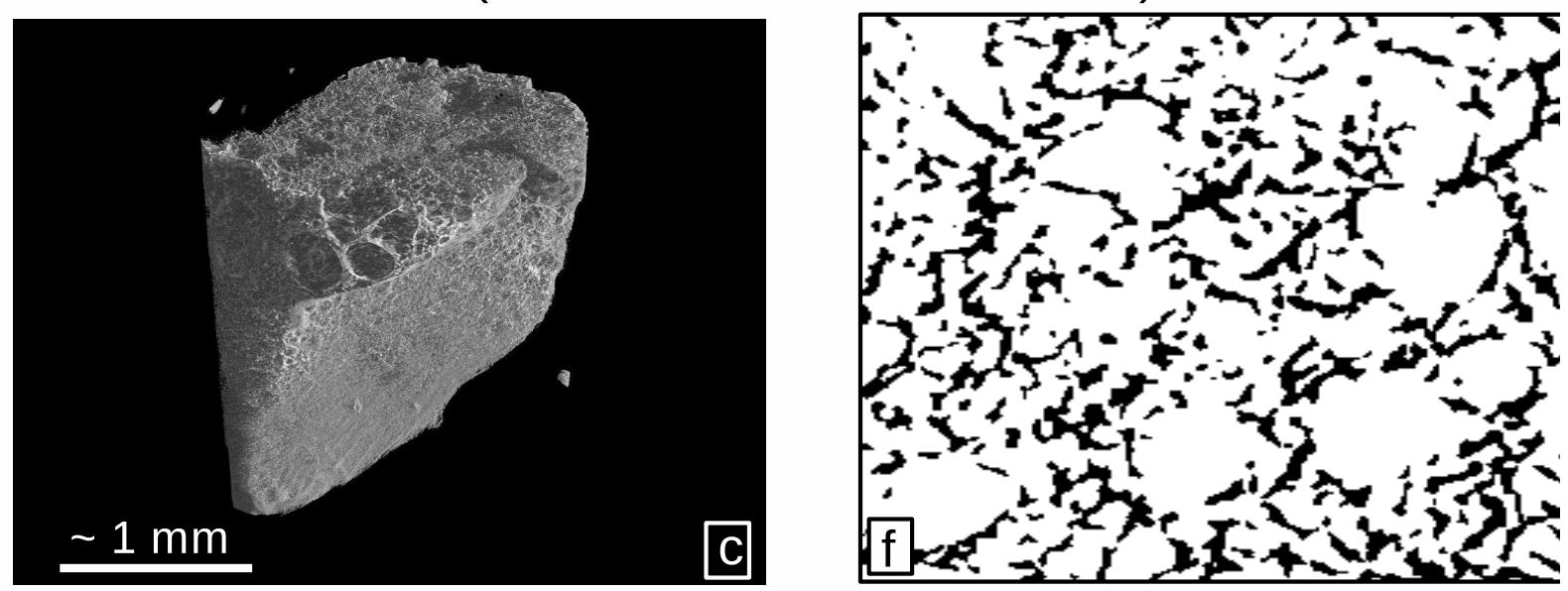


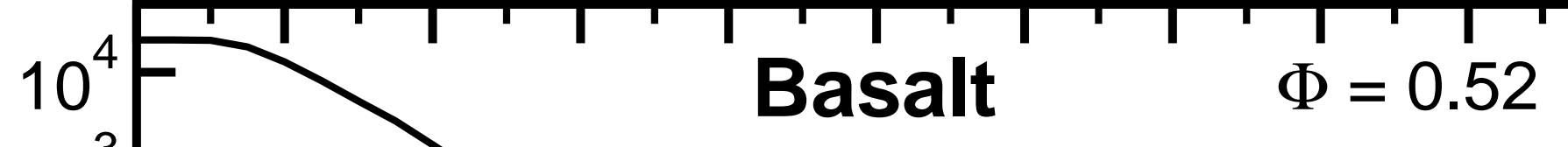

m

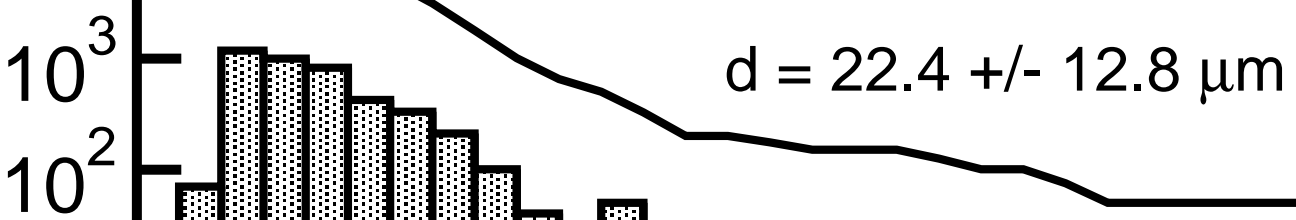

$10^{1}$

$10^{\circ}$

020

4060
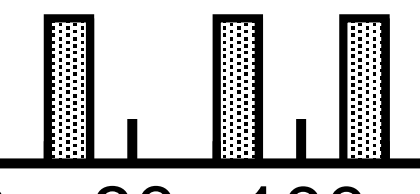

000100120140160180200
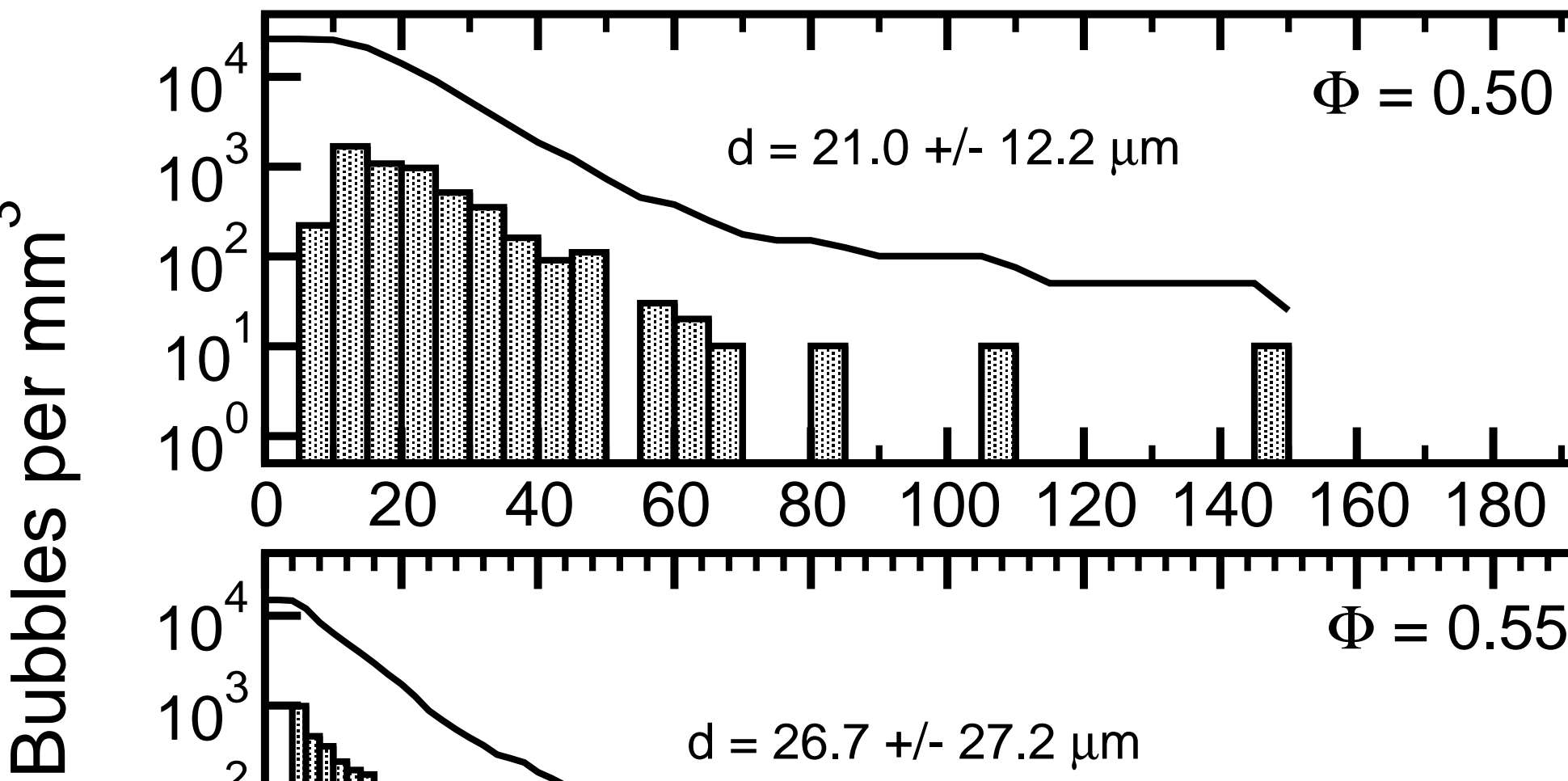

$10^{1}$

$10^{0}$

0
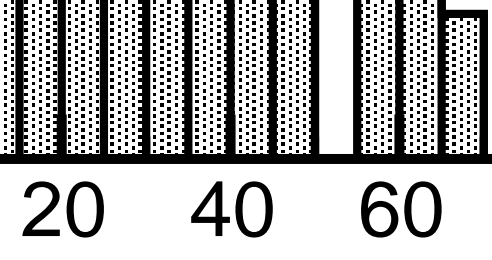

11

III

$60 \quad 80 \quad 100 \quad 120140 \quad 160 \quad 180 \quad 200$

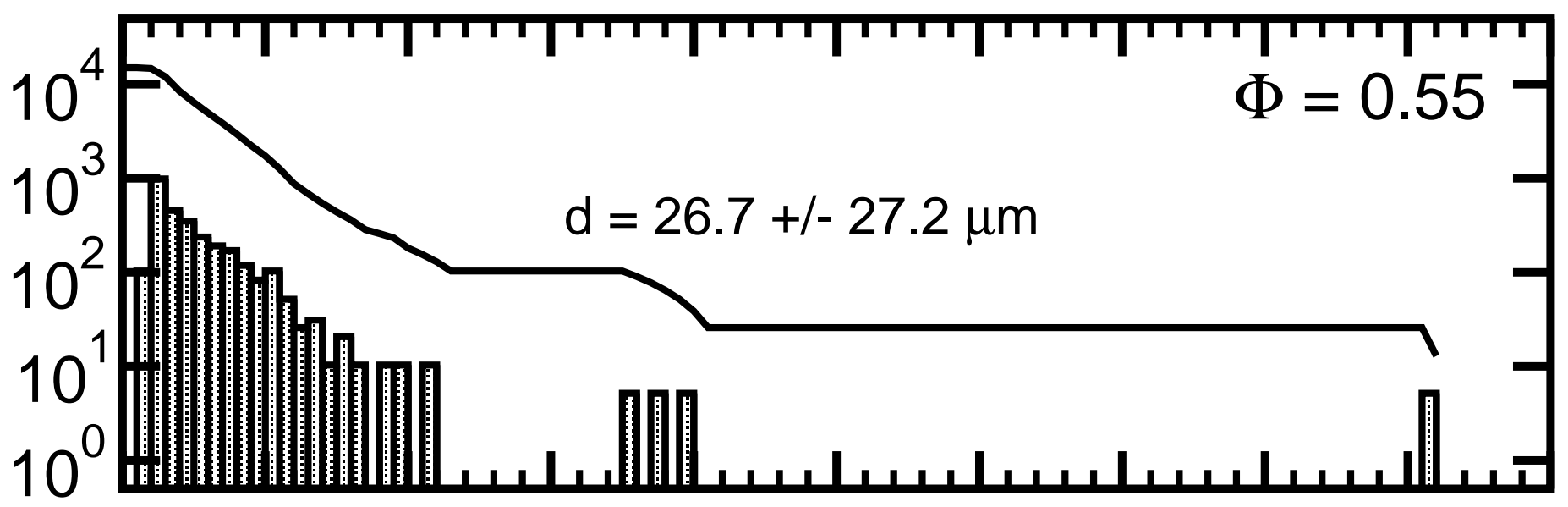

$0 \quad 50 \quad 100 \quad 150200250300 \quad 350 \quad 400450500$

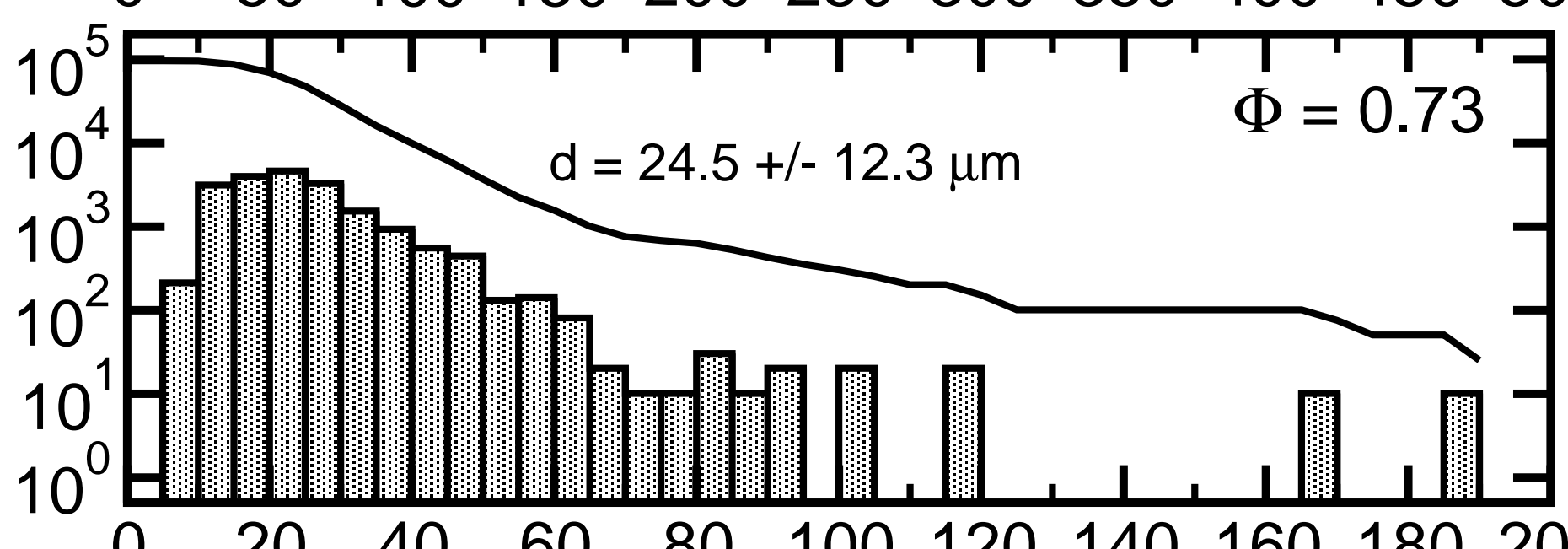

$\begin{array}{lllllllllll}0 & 20 & 40 & 60 & 80 & 100 & 120 & 140 & 160 & 180 & 200\end{array}$ Bubble diameter ( $\mu \mathrm{m})$ 


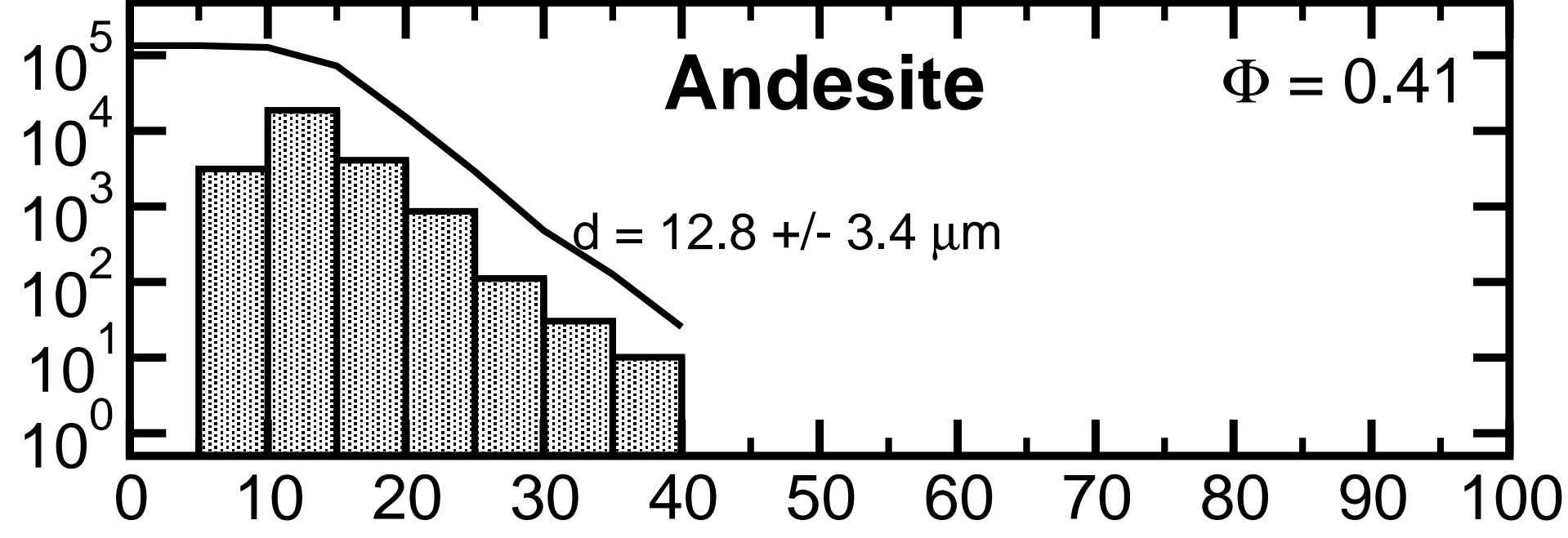

${ }^{n} \varepsilon$

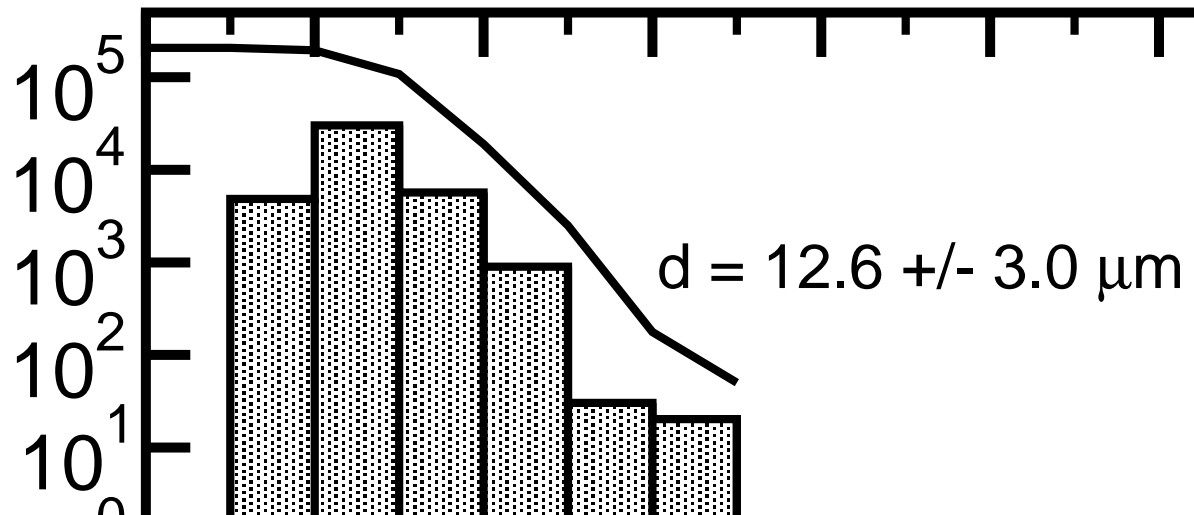

$\Phi=0.46$

$\begin{array}{lllllllllll}0 & 10 & 20 & 30 & 40 & 50 & 60 & 70 & 80 & 90 & 100\end{array}$
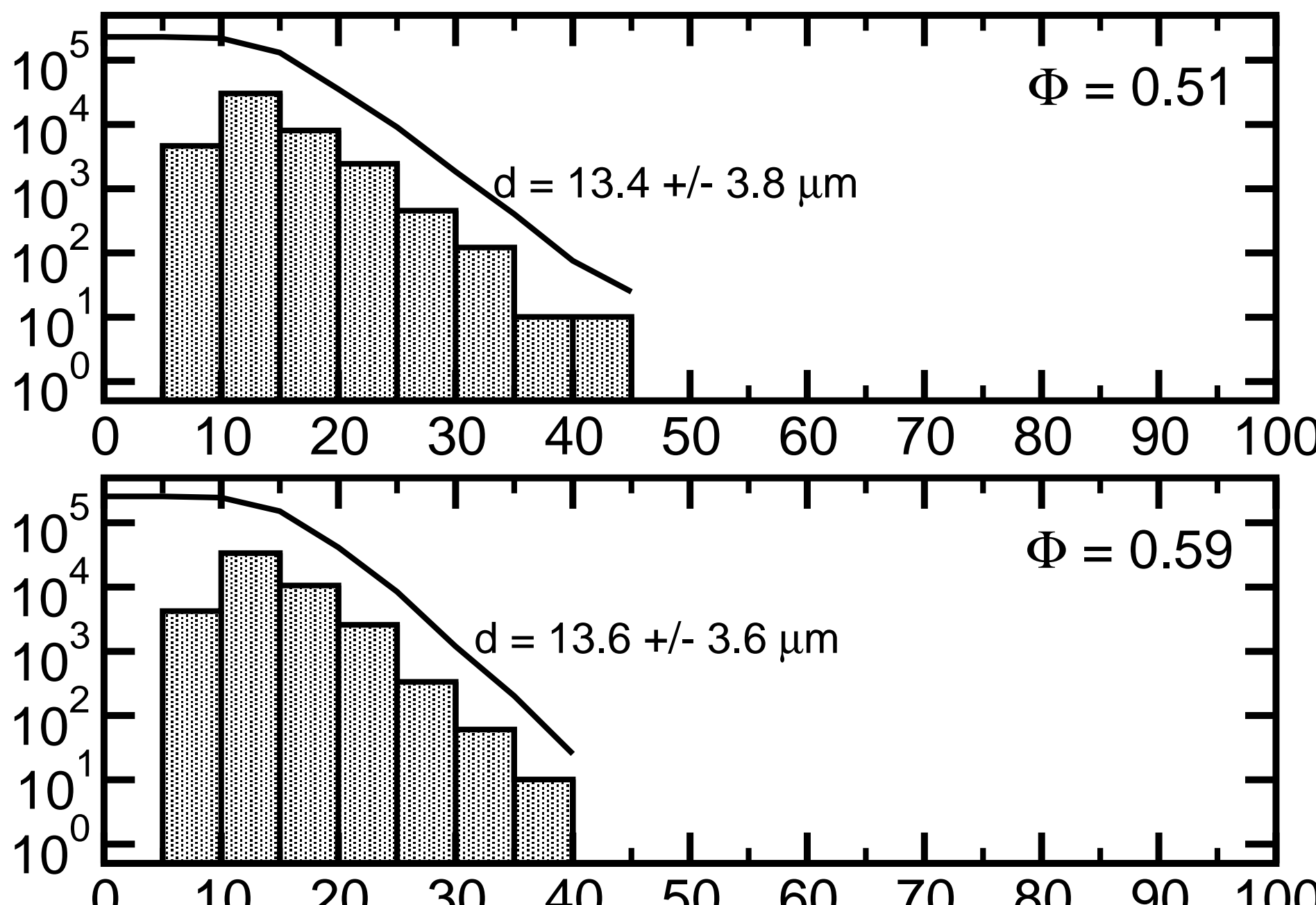

$\begin{array}{lllllllllll}0 & 10 & 20 & 30 & 40 & 50 & 60 & 70 & 80 & 90 & 100\end{array}$ Bubble diameter $(\mu \mathrm{m})$ 
m

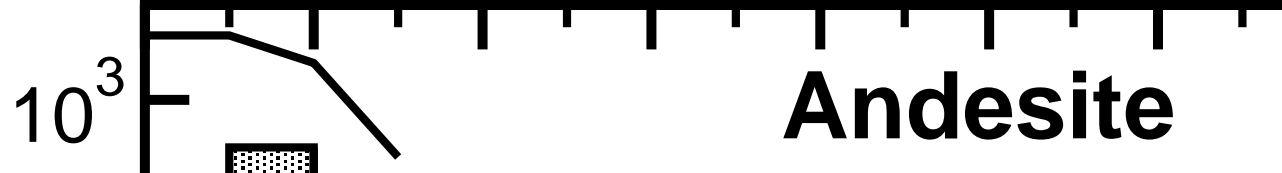

$\Phi=0.17$

$$
\mathrm{d}=8.0+/-2.1 \mu \mathrm{m}
$$

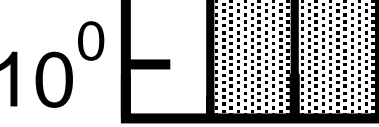

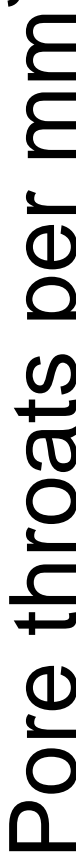

$d=8.0+/-2.2 \mu \mathrm{m}$

$10^{0}$

0

10

$\begin{array}{lllllllll}20 & 30 & 40 & 50 & 60 & 70 & 80 & 90 & 100\end{array}$

$10^{3}$

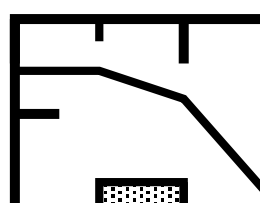

$10^{2}$

$10^{1}-$

$d=8.8+/-2.3 \mu m$

$\Phi=0.28$

$10^{0}$

$0 \quad 10$

$104 \overbrace{10}$ $\Phi=0.33$

$10^{3}$

$10^{2}$

$10^{1}$

$\mathrm{d}=8.0+/-2.1 \mu \mathrm{m}$

$10^{\circ} \mathrm{E}$

010

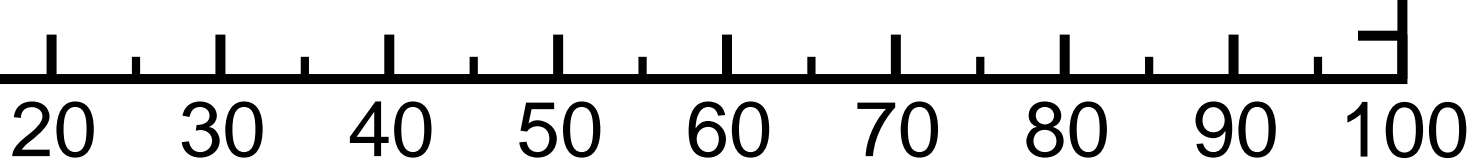
Pore throat diameter ( $\mu \mathrm{m})$ 


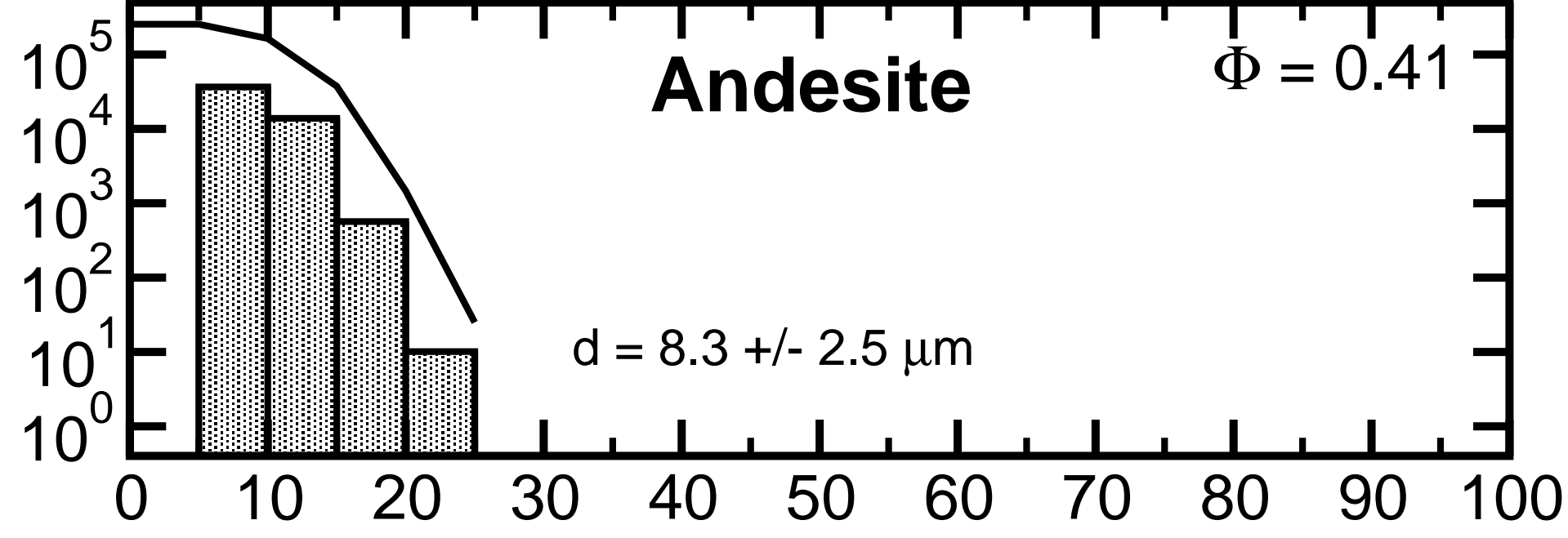

๓

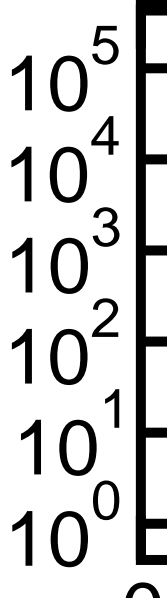

ه্

$\mathrm{d}=8.3+/-2.4 \mu \mathrm{m}$ $\Phi=0.45$
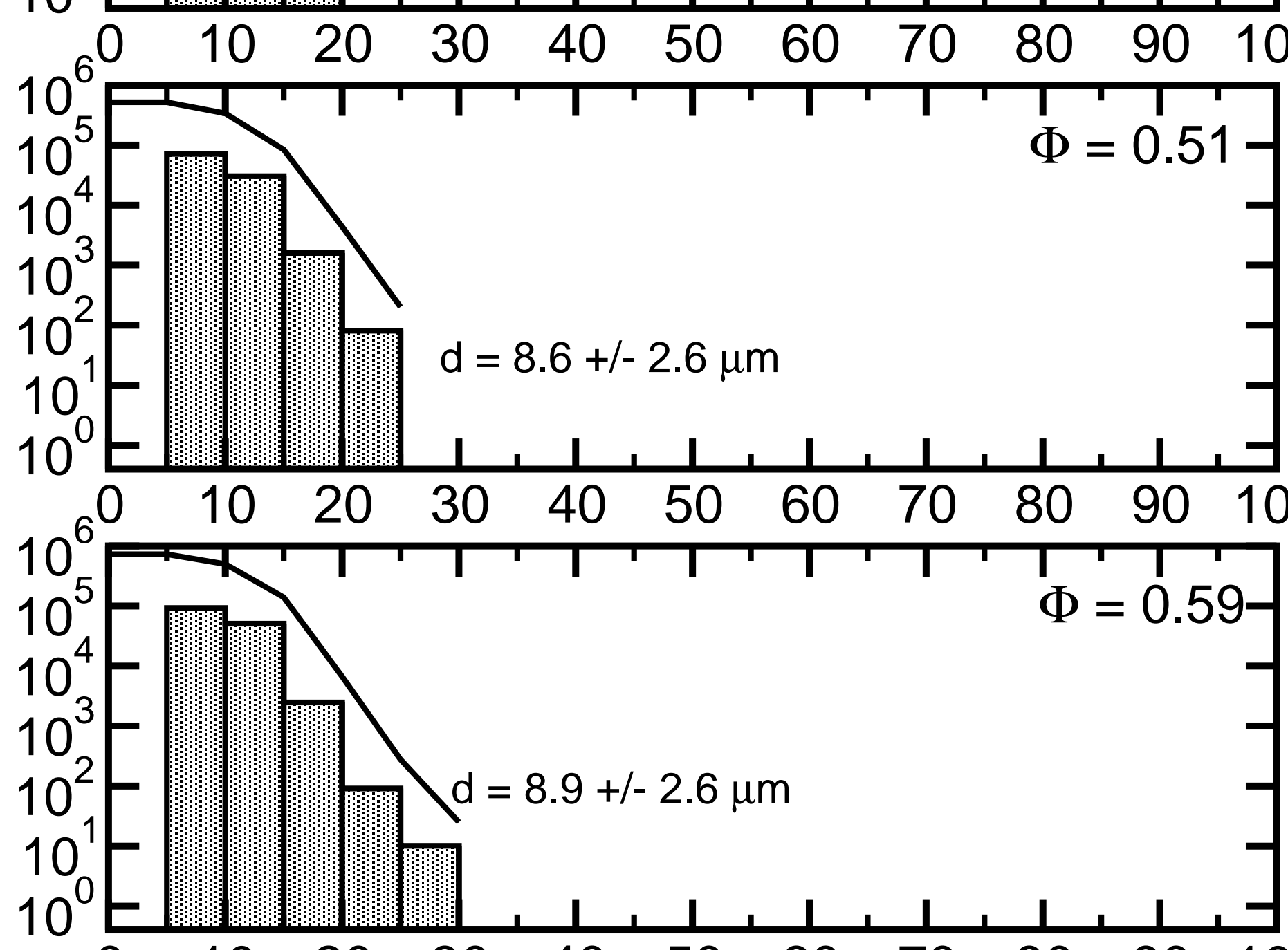

$\begin{array}{lllllllllll}0 & 10 & 20 & 30 & 40 & 50 & 60 & 70 & 80 & 90 & 100\end{array}$ Pore throat diameter ( $\mu \mathrm{m})$ 
$10^{6}$

$10^{5}$

$10^{4}$

$10^{3}$

$10^{2}$

$10^{1}$

$10^{\circ}$

$m$

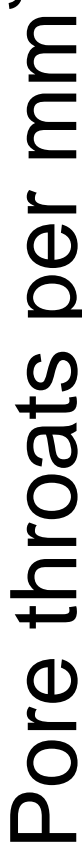

${ }_{10}{ }_{6}^{6} \begin{array}{ccccccccccccc} & 10 & 20 & 30 & 40 & 50 & 60 & 70 & 80 & 90 & 100\end{array}$

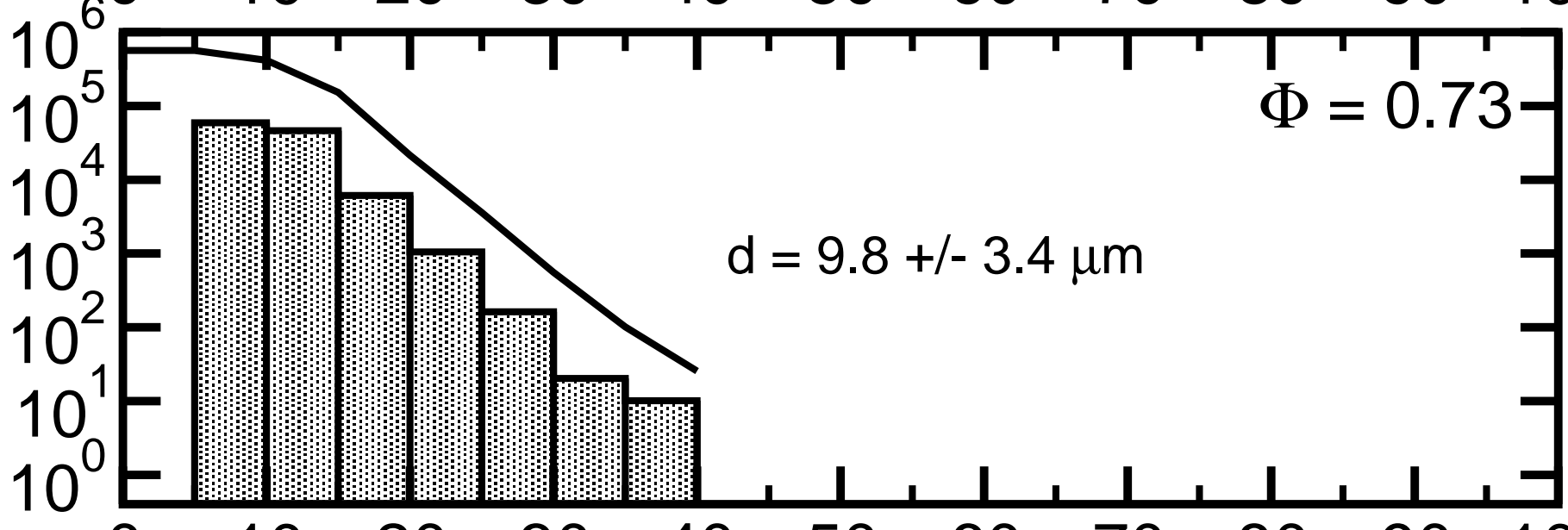

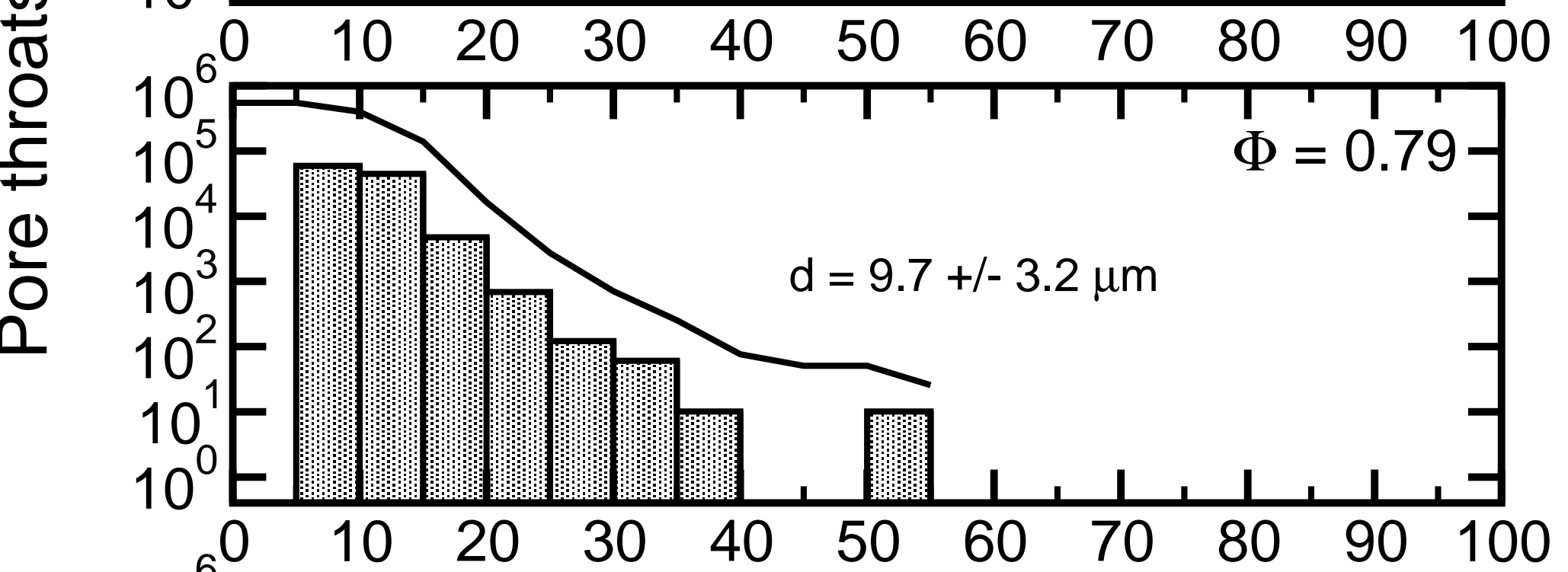

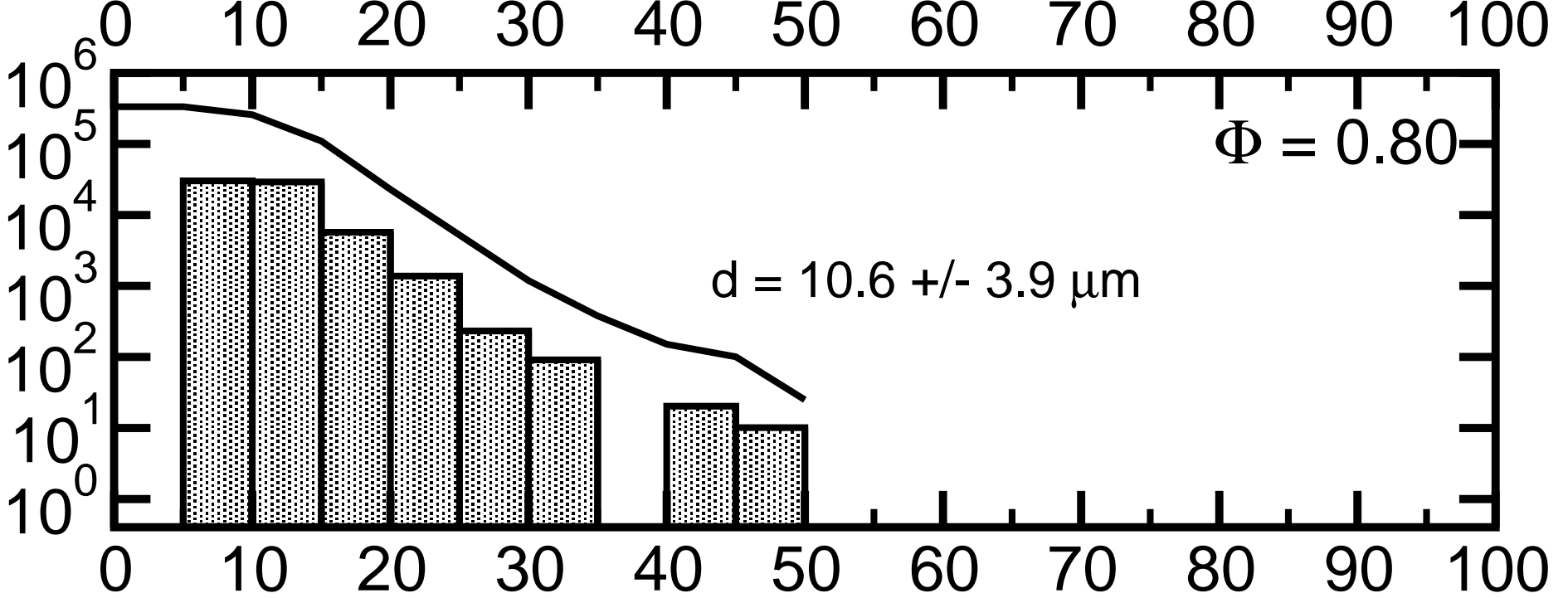
Pore throat diameter $(\mu \mathrm{m})$ 
$10^{4}$

$10^{3}-$

Trachyandesite
$\Phi=0.09$

m

$10^{2}$

$10^{1}-\sqrt{m} \quad d=8.2 \mu \mathrm{m}$

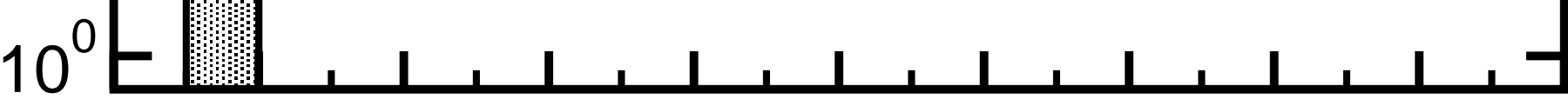

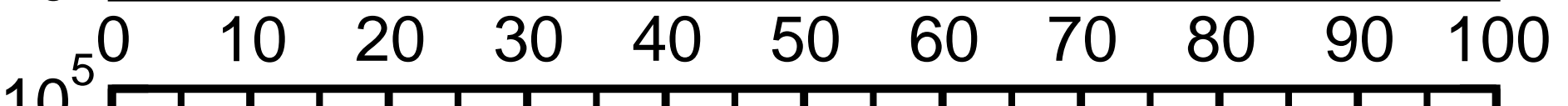

$\frac{E}{E}$

$10^{4}-$
$10^{3}-$

E $10^{2}-$

$10^{1}-$

$10^{0} \mathrm{E}$

$10^{5}$

$\begin{array}{lll}10 & 20 & 30\end{array}$

$40 \quad 50$

6070

$\Phi=0.35$

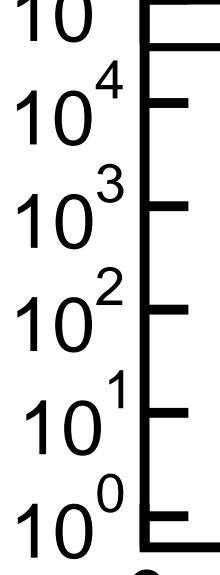

$d=17.8+/-7.1 \mu \mathrm{m}$

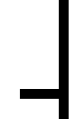

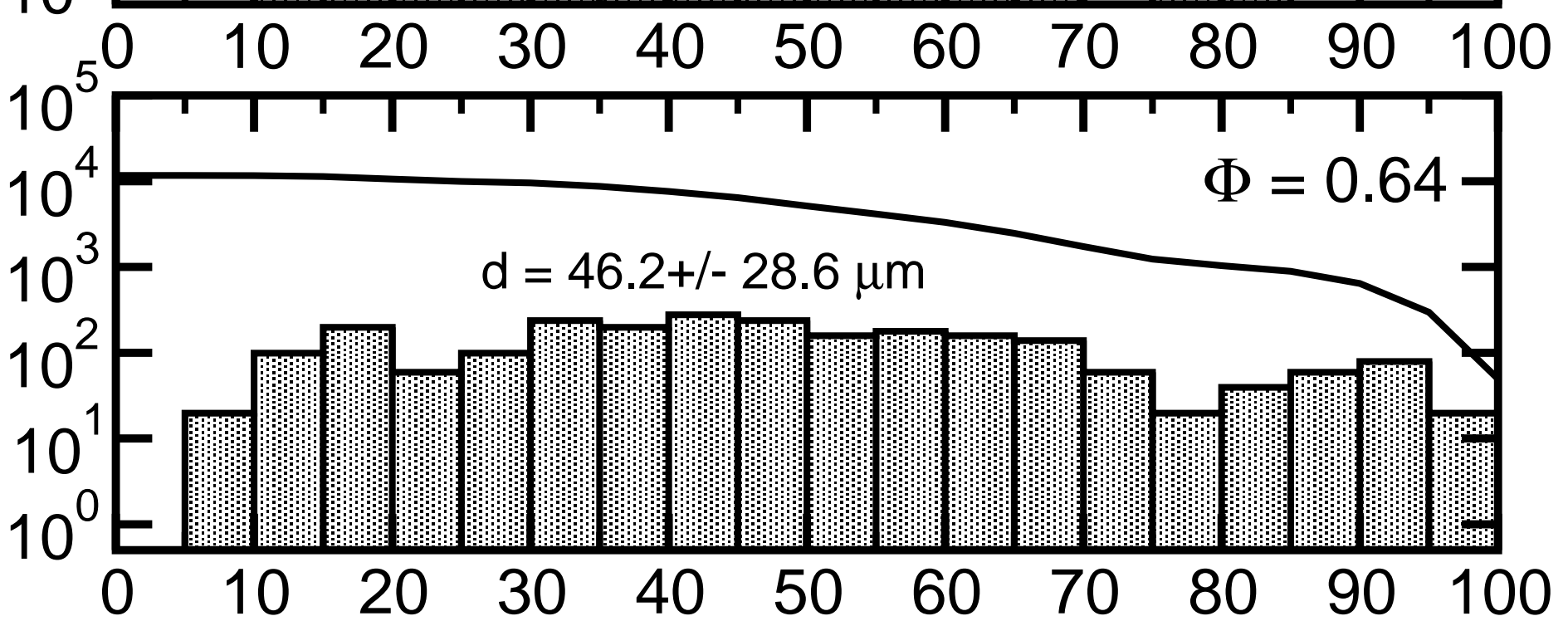
Bubble diameter $(\mu \mathrm{m})$ 


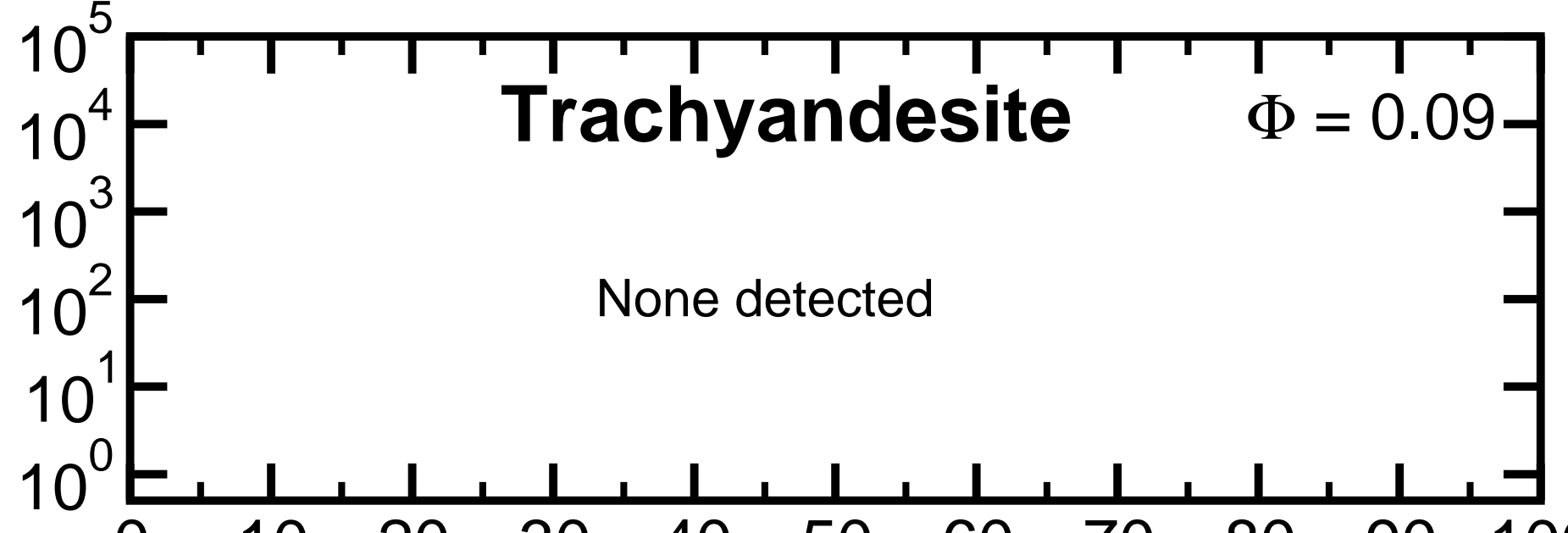

$m$

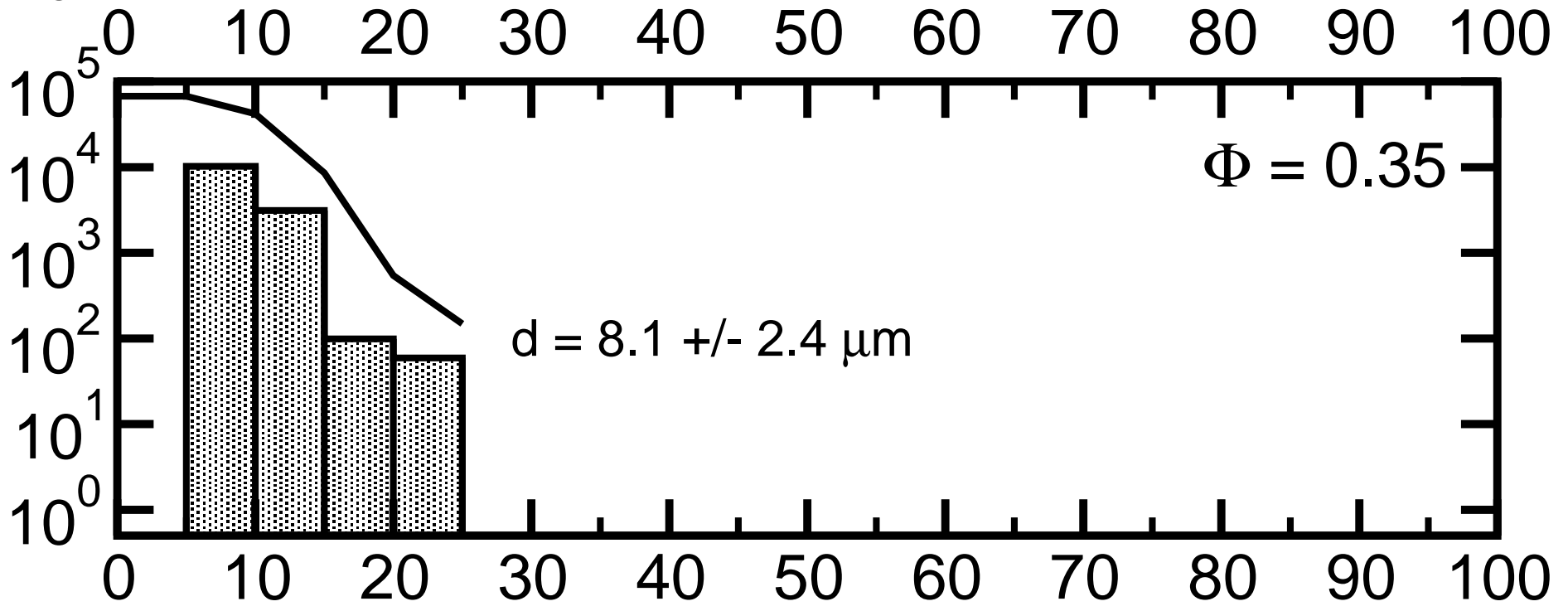

$\frac{0}{5}$

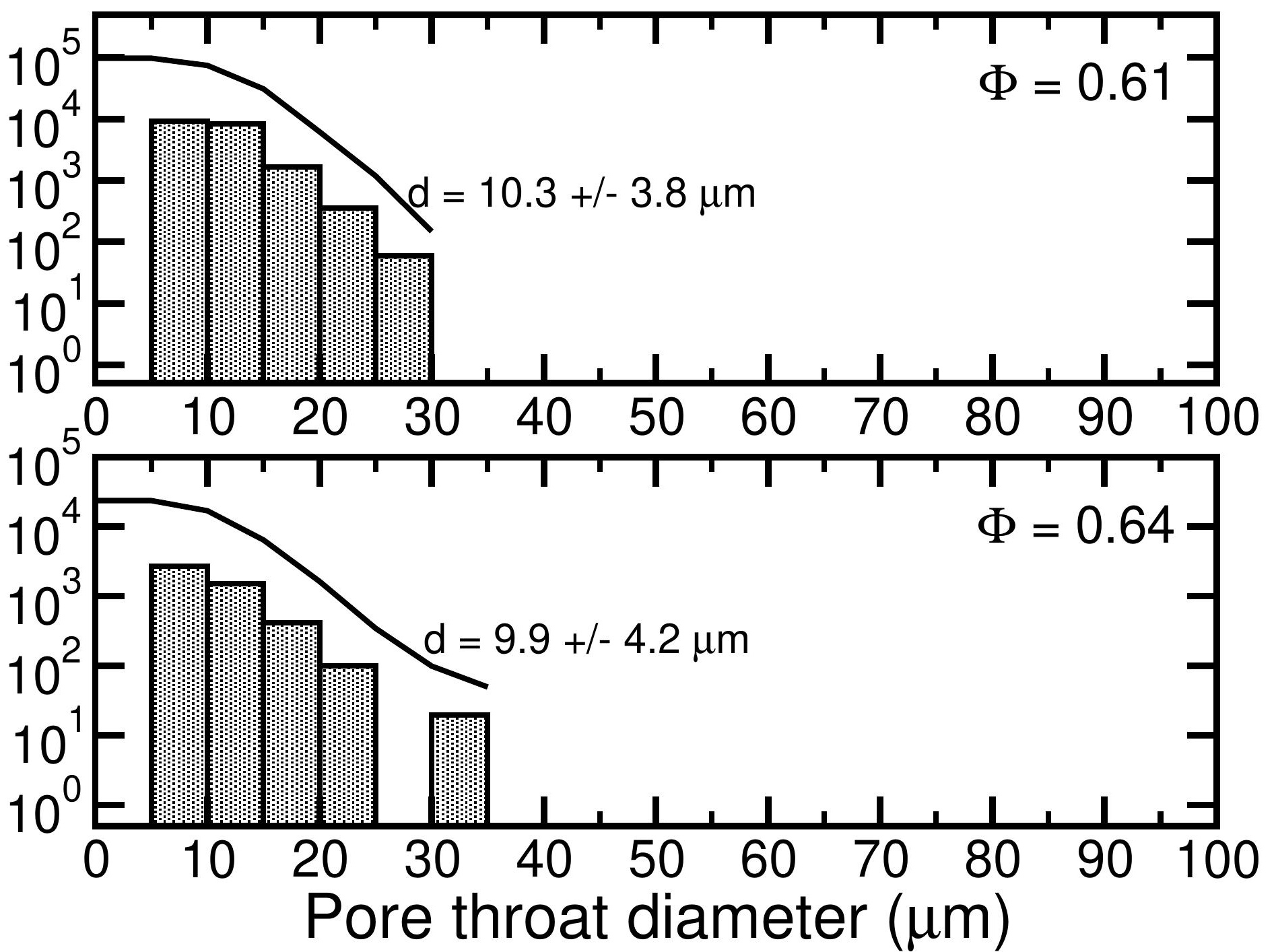




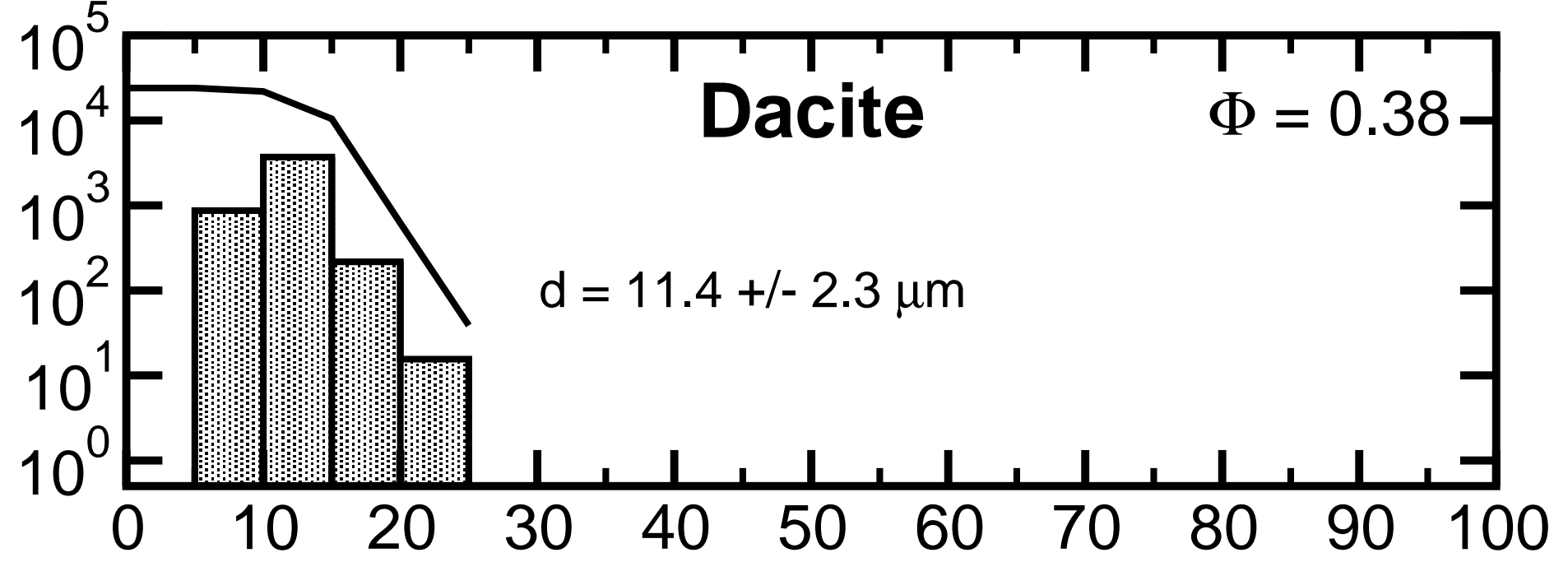

r.

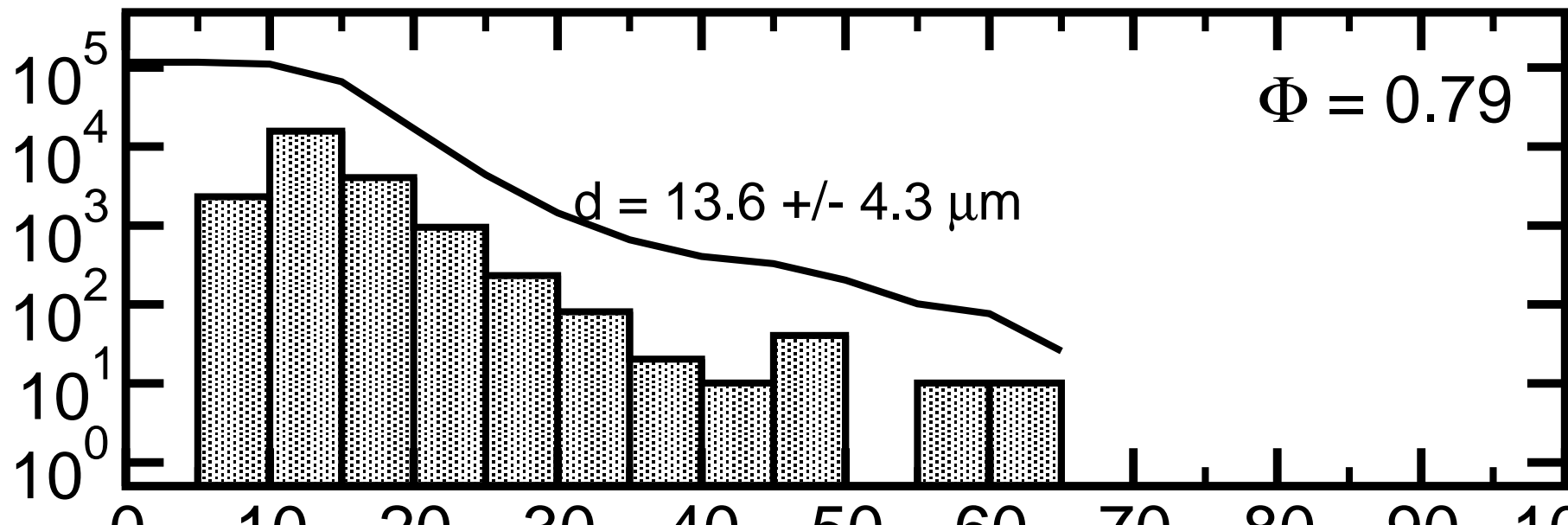

웅

0

$\begin{array}{lll}10 & 20 & 30\end{array}$ $40 \quad 50 \quad 60$ T1
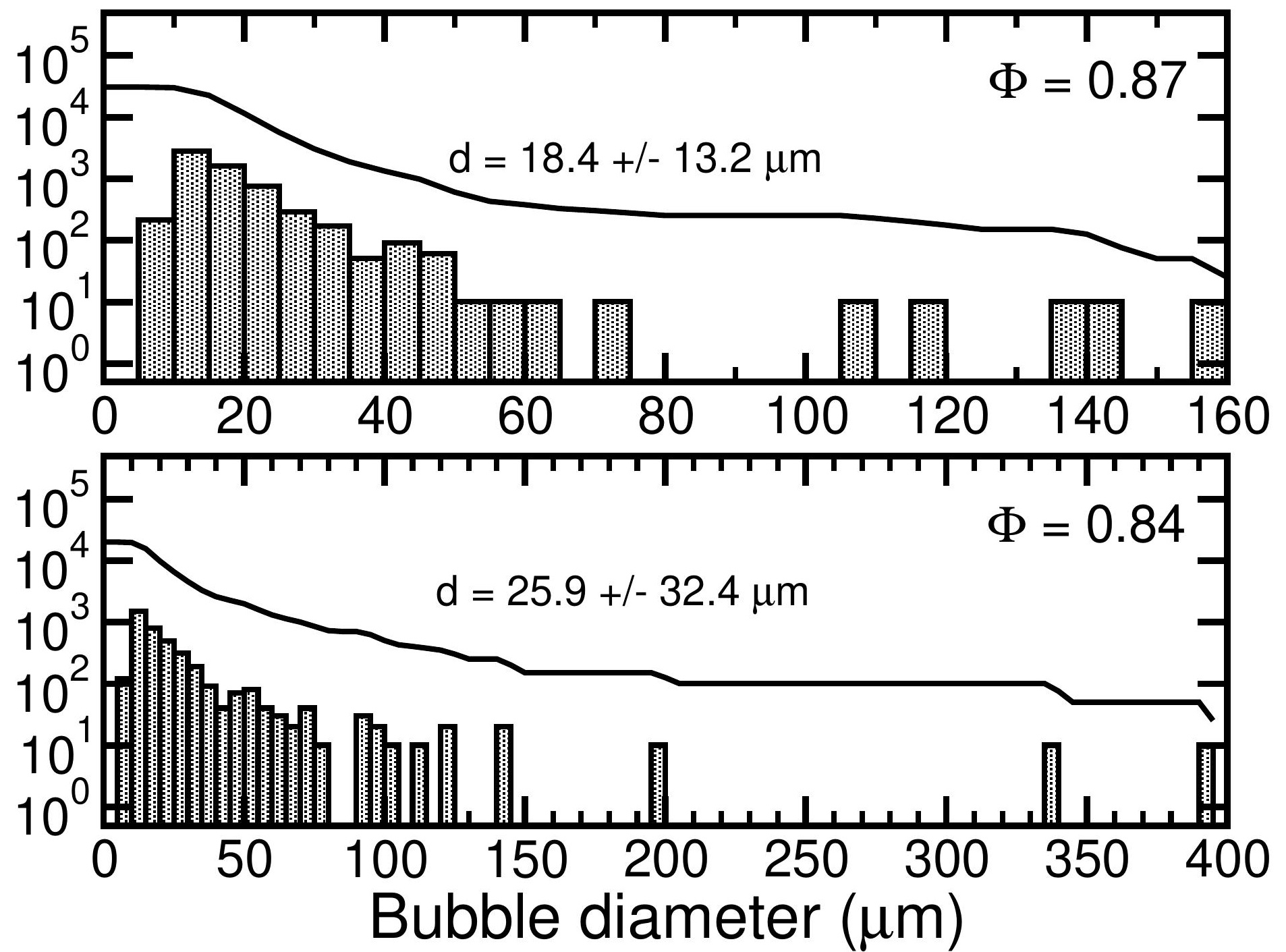


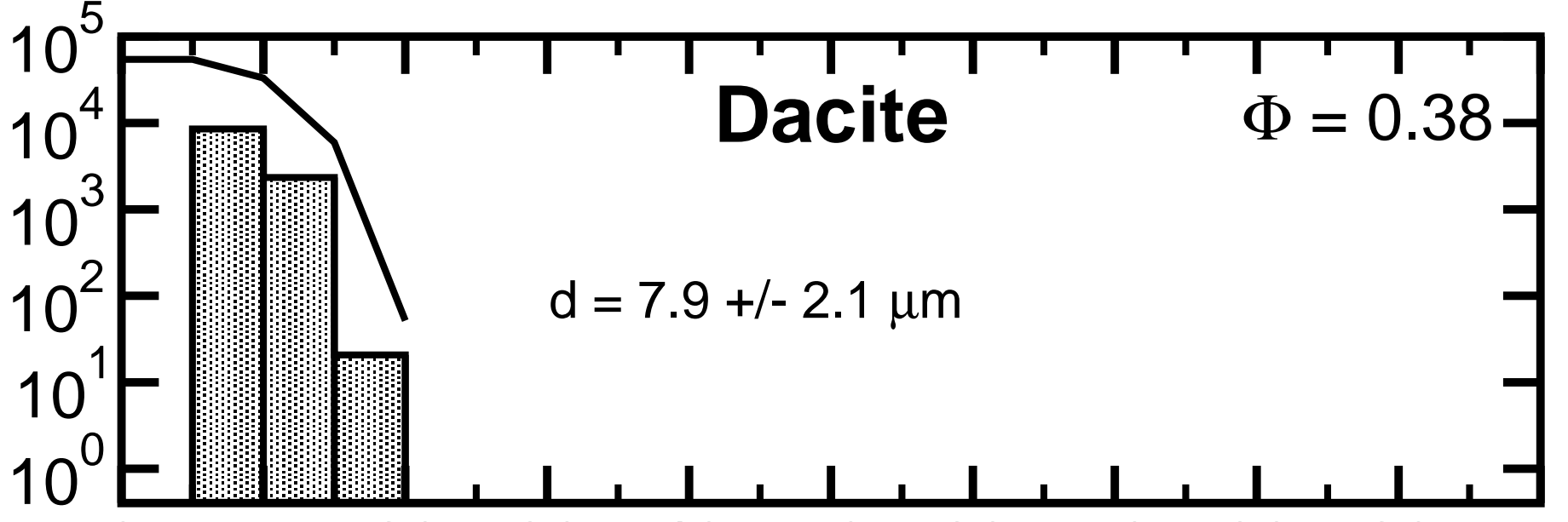

m $10^{6} \begin{array}{lllllllllll}0 & 10 & 20 & 30 & 40 & 50 & 60 & 70 & 80 & 90 & 100\end{array}$

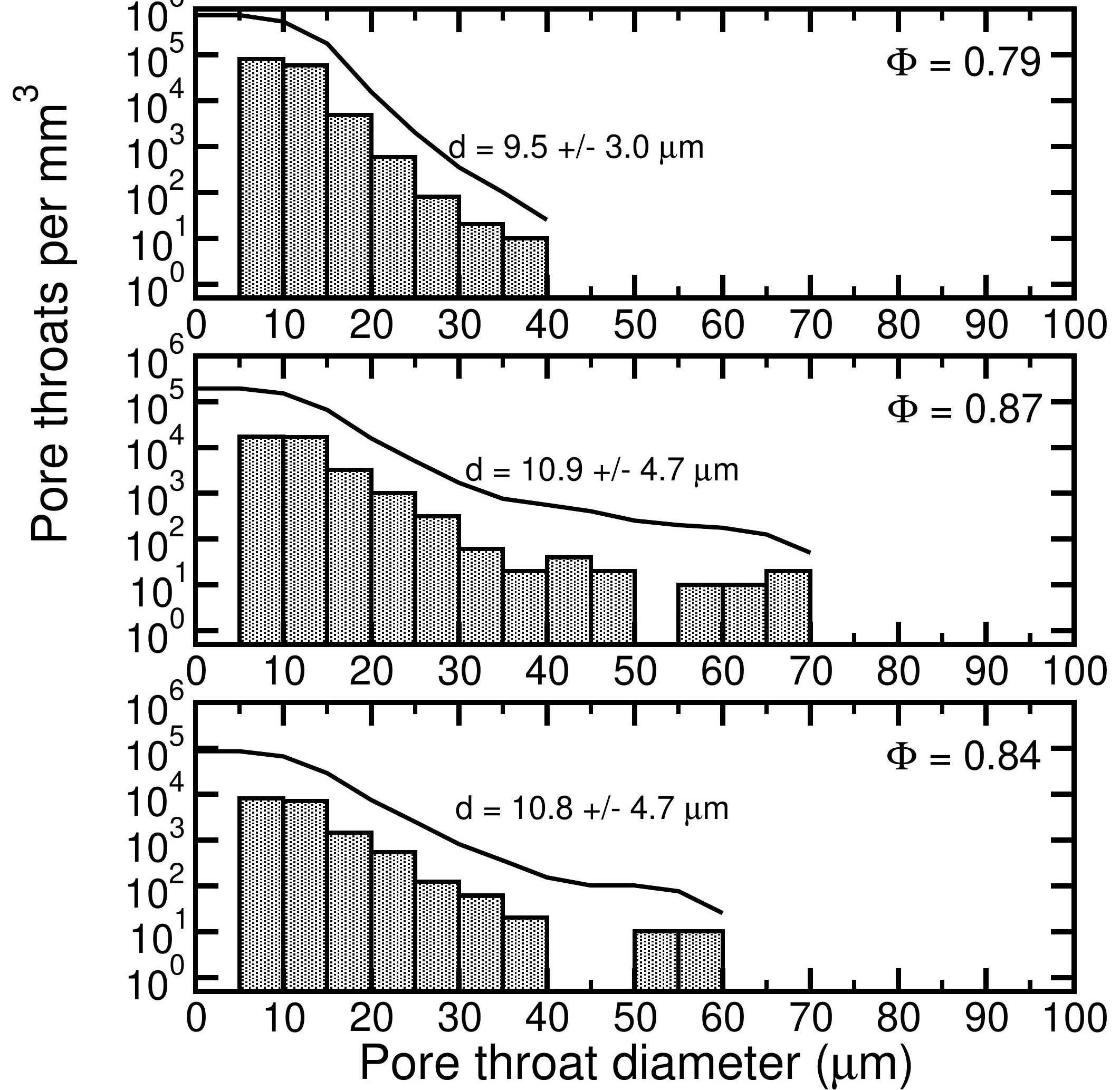




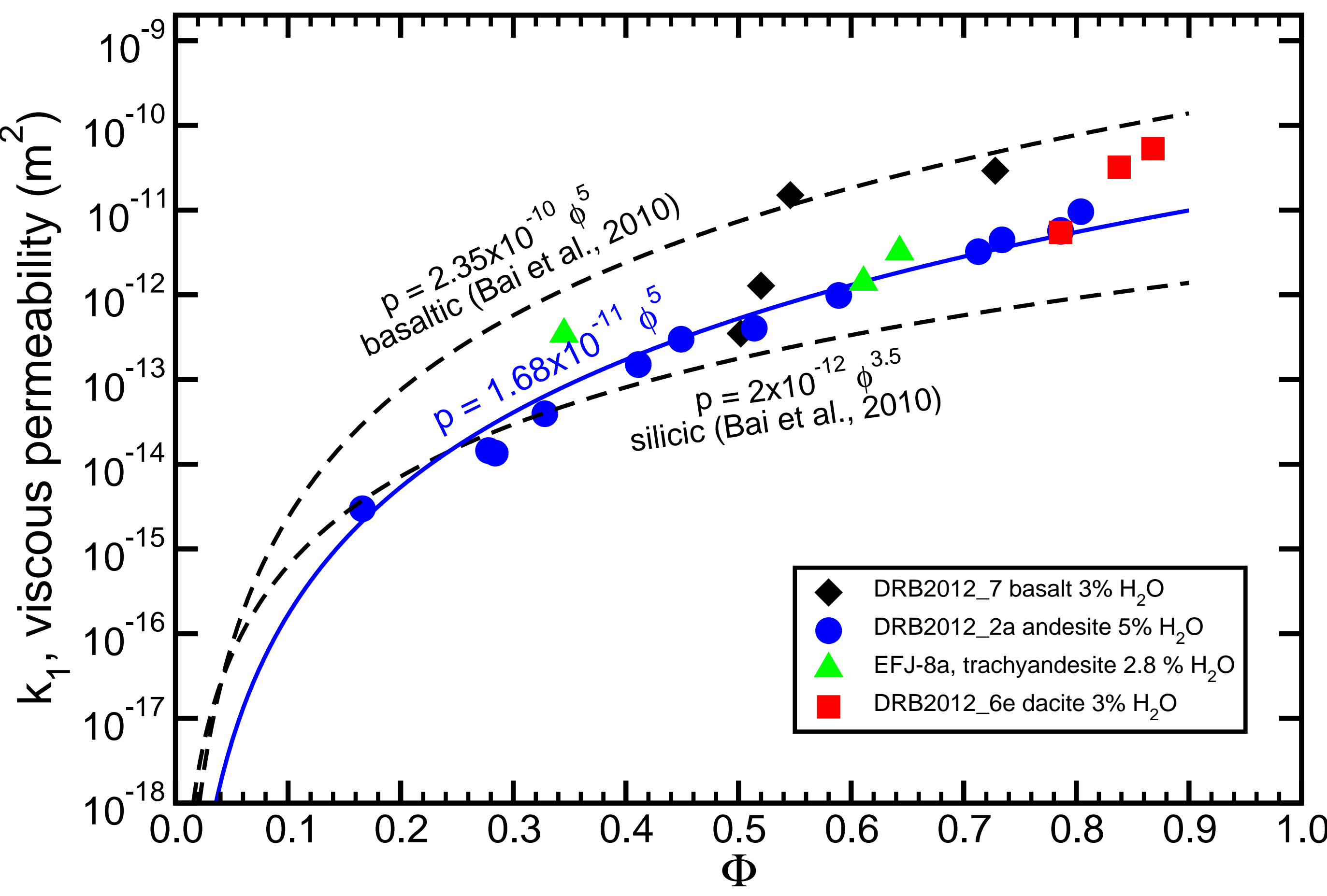




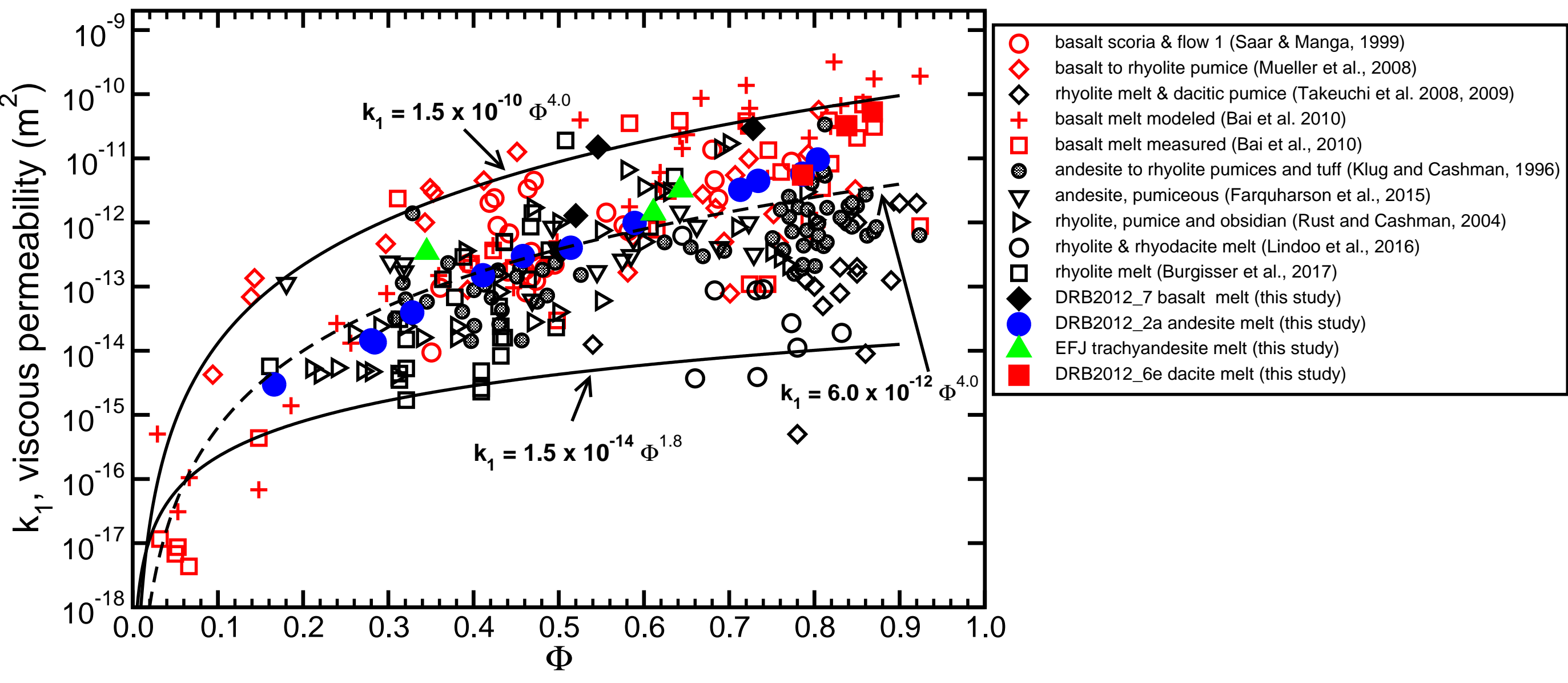




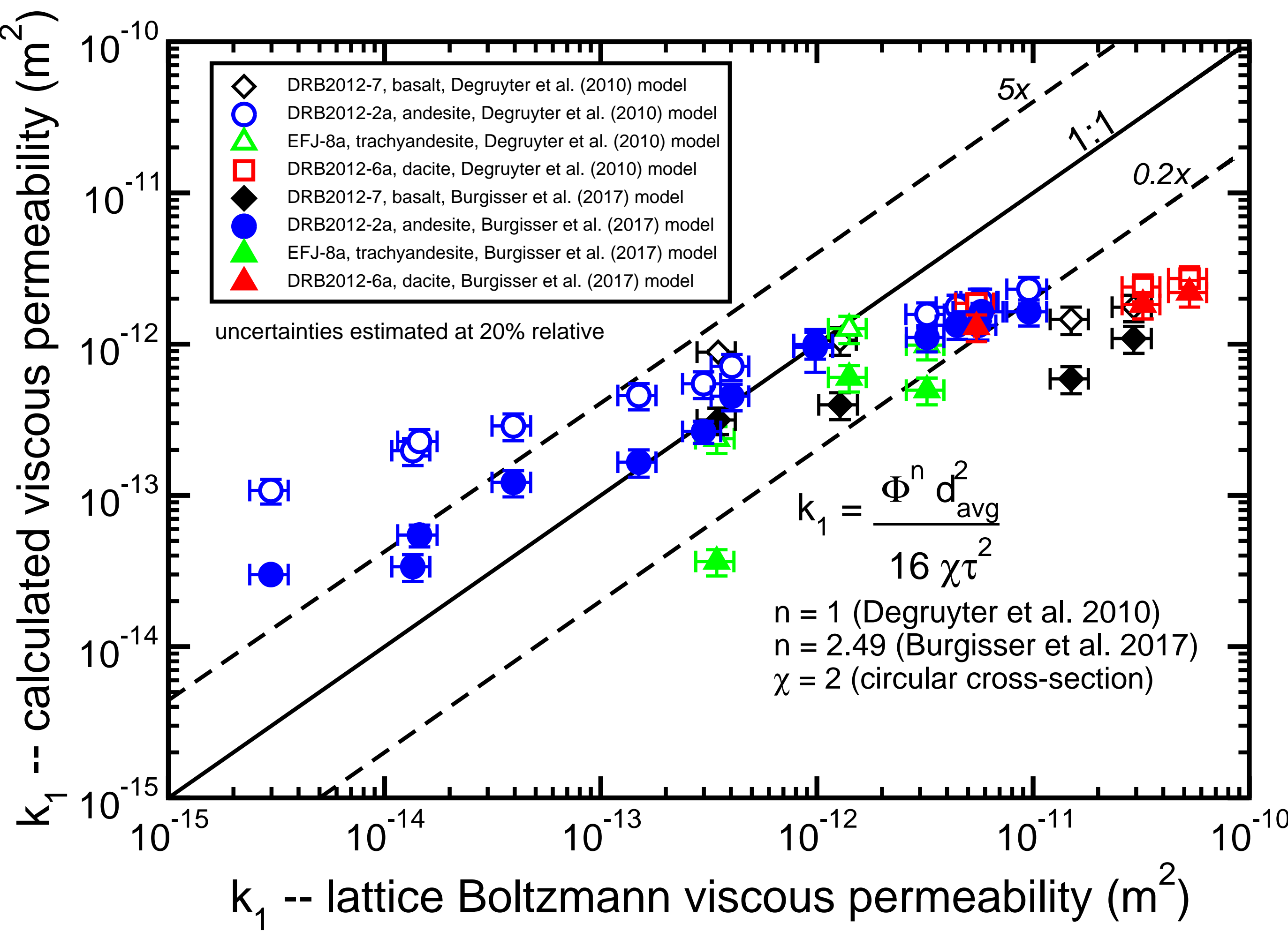




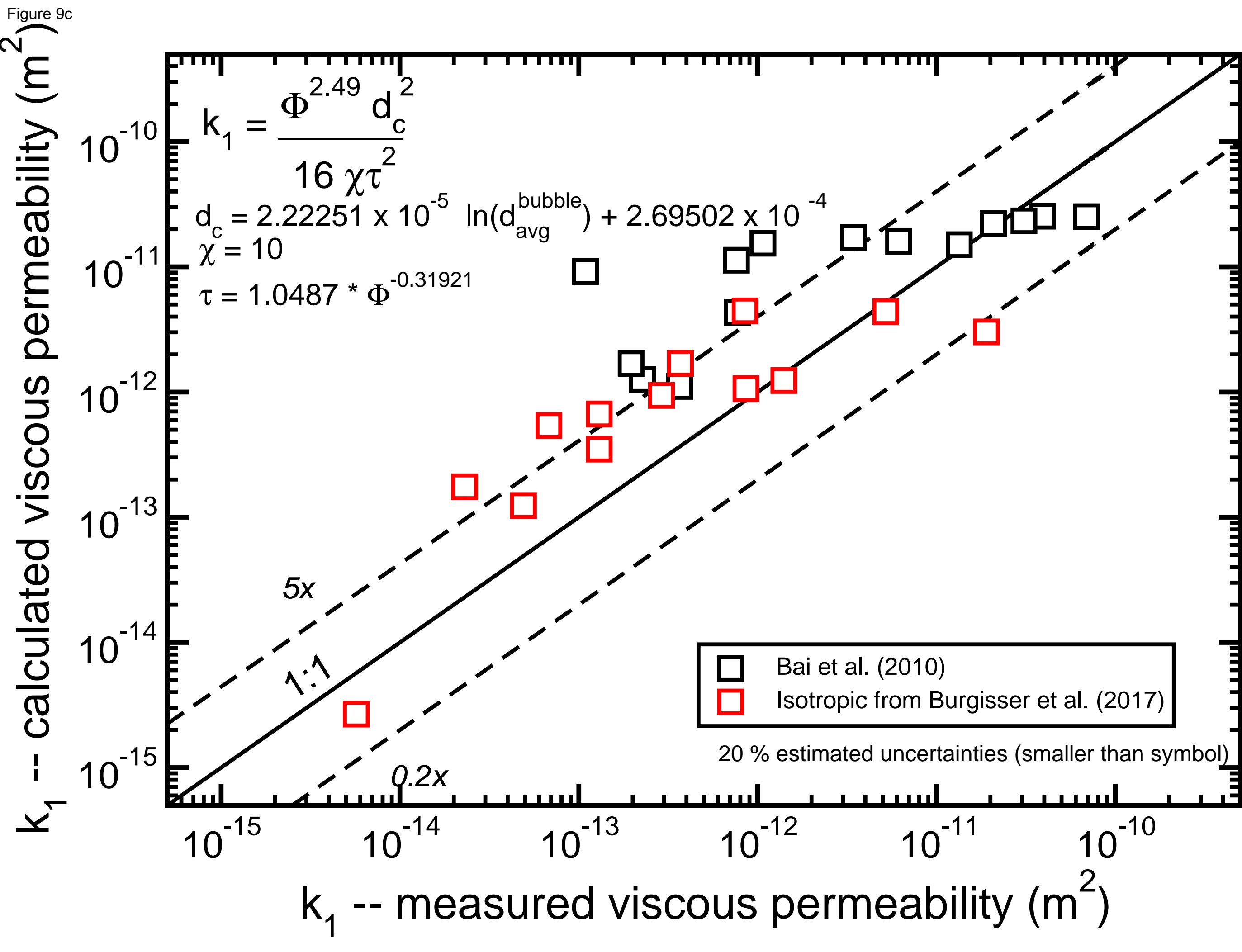


Sheet1

Table 1: Starting compositions

\begin{tabular}{|c|c|c|c|c|}
\hline & MORB' ${ }^{1}$ & $\begin{array}{l}\text { Andesite }^{2} \\
\text { AT-29 }\end{array}$ & $\begin{array}{c}\text { Trachyandesite }^{3} \\
\text { EFJ }\end{array}$ & $\begin{array}{l}\text { Dacite }^{4} \\
\text { AT-150 }\end{array}$ \\
\hline $\mathrm{SiO}_{2}$ & 49.7 & 56.8 & 58.5 & 64.1 \\
\hline $\mathrm{TiO}_{2}$ & 1.41 & 1.01 & 1.55 & 0.61 \\
\hline $\mathrm{Al}_{2} \mathrm{O}_{3}$ & 16.1 & 16.9 & 14.6 & 16.4 \\
\hline $\mathrm{FeO}^{\text {total }}$ & 10.3 & 8.03 & 9.35 & 5.19 \\
\hline MnO & 0.17 & 0.17 & 0.24 & \\
\hline $\mathrm{MgO}$ & 7.8 & 3.09 & 2.97 & 2.00 \\
\hline $\mathrm{CaO}$ & 10.8 & 7.05 & 4.84 & 4.78 \\
\hline $\mathrm{Na}_{2} \mathrm{O}$ & 2.65 & 3.99 & 5.19 & 4.45 \\
\hline $\mathrm{K}_{2} \mathrm{O}$ & 0.1 & 2.05 & 1.83 & 1.98 \\
\hline $\mathrm{P}_{2} \mathrm{O}_{5}$ & 0.11 & 0.28 & 0.47 & \\
\hline Total & 99.14 & 99.37 & 99.52 & 99.51 \\
\hline $\mathrm{H}_{2} \mathrm{O}$ & 2.9 & 5.0 & 2.8 & 3.0 \\
\hline Viscosity (Pa s) ${ }^{5}$ & $14 / 234$ & $32 / 6685$ & $115 / 7505$ & $359 / 61126$ \\
\hline
\end{tabular}

${ }^{1}$ Fortin et al. (2015)

${ }^{2}$ AT-29 from Baker and Eggler (1987)

${ }^{3}$ Average of compositions in LaRue, M.Sc. Thesis (2012)

${ }^{4}$ Liu et al. (2007)

$51100{ }^{\circ} \mathrm{C}$ melt viscosity at the initial water concentration followed by viscosity

in the anhdyrous melt calculated following Giordano et al. (2008) 
Table 1: Starting compositions

\begin{tabular}{|c|c|c|c|}
\hline & MORB $^{1}$ & $\begin{array}{l}\text { Andesite }^{2} \\
\text { AT-29 }\end{array}$ & $\begin{array}{c}\text { Trachyandesite }^{3} \\
\text { EFJ }\end{array}$ \\
\hline $\mathrm{SiO}_{2}$ & 49.7 & 56.8 & 58.5 \\
\hline $\mathrm{TiO}_{2}$ & 1.41 & 1.01 & 1.55 \\
\hline $\mathrm{Al}_{2} \mathrm{O}_{3}$ & 16.1 & 16.9 & 14.6 \\
\hline $\mathrm{FeO}^{\text {total }}$ & 10.3 & 8.03 & 9.35 \\
\hline MnO & 0.17 & 0.17 & 0.24 \\
\hline $\mathrm{MgO}$ & 7.8 & 3.09 & 2.97 \\
\hline $\mathrm{CaO}$ & 10.8 & 7.05 & 4.84 \\
\hline $\mathrm{Na}_{2} \mathrm{O}$ & 2.65 & 3.99 & 5.19 \\
\hline $\mathrm{K}_{2} \mathrm{O}$ & 0.1 & 2.05 & 1.83 \\
\hline $\mathrm{P}_{2} \mathrm{O}_{5}$ & 0.11 & 0.28 & 0.47 \\
\hline Total & 99.14 & 99.37 & 99.52 \\
\hline $\mathrm{H}_{2} \mathrm{O}$ & 2.9 & 5.0 & 2.8 \\
\hline Viscosity (Pa s) ${ }^{5}$ & $14 / 234$ & $32 / 6685$ & $115 / 7505$ \\
\hline
\end{tabular}

${ }^{1}$ Fortin et al. (2015)

${ }^{2}$ AT-29 from Baker and Eggler (1987)

${ }^{3}$ Average of compositions in LaRue, M.Sc. Thesis (2012)

${ }^{4}$ Liu et al. (2007)

${ }^{5} 1100{ }^{\circ} \mathrm{C}$ melt viscosity at the initial water concentration followed by viscosity in the anhdyrous melt calculated following Giordano et al. (2008) 
Sheet1

\begin{tabular}{c} 
Dacite $^{4}$ \\
AT-150 \\
\hline 64.1 \\
0.61 \\
16.4 \\
5.19 \\
\\
2.00 \\
4.78 \\
4.45 \\
1.98 \\
\\
99.51 \\
3.0 \\
\\
\end{tabular}

Page 2 
Sample Volun

\section{Basalt, $3 \% \mathrm{H}_{2} \mathrm{O}, \Delta P=68 \mathrm{MPa}^{8}$
DRB2012-07a-2 0.0989}

DRB2012-07a-3 $\quad 0.0989 \quad 0.52$

DRB2012-7f-10

0.1931

0.50
0.55
0.73

Onnected
Porosity

Trachyandesite, $2.8 \% \mathrm{H}_{2} \mathrm{O}, \triangle \mathrm{P}=35 \mathrm{MPa}$

$\begin{array}{lll}\text { EFJ-8a-06 } & 0.0506 & 0.09 \\ \text { EFJ-8a-07 } & 0.0506 & 0.35 \\ \text { EFJ-8a } 08 & 0.0506 & 0.6 \\ \text { EFJ-8a-09 } & 0.0506 & 0.64 \\ & \\ & \end{array}$

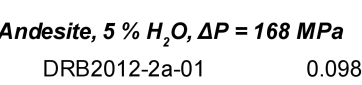

$\begin{array}{lll}\text { DRB2012-2a-01 } & 0.0989 & 0.17 \\ \text { DRB2212-2a-08 } & 0.0989 & 0.22 \\ \text { DRB2012-2a-09 } & 0.0989 & 0.28 \\ \text { DRB2012-2a-10 } & 0.0989 & 0.33 \\ \text { DRB2012-2a-11 } & 0.0989 & 0.41 \\ \text { DRB2012-2a-12 } & 0.0989 & 0.46 \\ \text { DRB2012-2a-13 } & 0.0989 & 0.51 \\ \text { DRB2012-2a-14 } & 0.0989 & 0.59 \\ \text { DRB2012-2a-15 } & 0.0989 & 0.77 \\ \text { DRB2012-2a-16 } & 0.0989 & 0.73 \\ \text { DRB2012-2a-17 } & 0.0989 & 0.79 \\ \text { DRB2212-2a-18 } & 0.0989 & 0.79 \\ \text { DRB2012-2a-19 } & 0.0989 & 0.80\end{array}$

Dacite, $3 \% \mathrm{H}_{2} \mathrm{O}, \triangle \mathrm{P}=74 \mathrm{MPa}$

$\begin{array}{lll}\text { DRB2012-6e-7 } & 0.1931 & 0.37 \\ \text { DRB2012-6e-8 } & 0.0989 & 0.79 \\ \text { DRB2012-6e-9 } & 0.0989 & 0.87 \\ \text { DRB2012-6e-10 } & 0.0989 & 0.84\end{array}$

0.55 $\begin{array}{ll}0.52 & 4309 \\ 0.50 & 5290\end{array}$

5290
3009
19228

6403
8092
7706

8092
7706
72157

1.486
1.530
2.561
3.753

Average bubble Median bubble Average throat
diameter $(m)$ $\begin{gathered}\text { Median throat } \\ \text { diameter }(m)\end{gathered} \begin{gathered}\text { diameter }(m) \\ \text { diameter }(m)\end{gathered}$

$\underset{(m)}{(m)}$

$\begin{array}{llll}2.24 \times 10^{-5} & 1.92 \times 10^{-5} & 1.08 \times 10^{-5} & 8.2 \times 10^{-6} \\ 2.10 \times 10^{-5} & 1.73 \times 10^{-5} & 1.01 \times 10^{-5} & 8.2 \times 10^{-6}\end{array}$

$2.10 \times 10^{-5}$
$2.67 \times 10^{-5}$

$1.73 \times 10^{-5}$
$1.91 \times 10^{-5}$

$1.01 \times 10^{-5}$

$8.2 \times 10^{-6}$
$8.2 \times 10^{-6}$

$\begin{array}{lll}5.36 \times 10^{-5} & 2.10 \times 10^{3} & 1.33 \pm 0.10 \\ 536 \times 10^{-5} & 2.10 & \end{array}$

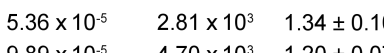

$6.8 \pm 6.2$

$1.28 \times 10^{-12}$
$3.49 \times 10^{-13}$
$1.50 \times 10^{-1}$

$1.50 \times 1011$

$8.20 \times 10^{-6}$

$1.78 \times 10^{-5}$
$3.59 \times 10^{-5}$

$1.73 \times 10^{-5}$

$18.0 \times 100$

\section{$8.2 \times 10^{\circ}$}

$216 \times 10^{-5} \quad 452 \times 1$

$\begin{array}{llll}0.33 & 8989 & 13493 & 1.501 \\ 0.61 & 6934 & 19419 & 2.801 \\ 0.64 & 2331 & 474 & 2.034\end{array}$

$4.62 \times 10^{-5}$

$4.44 \times 10^{-5}$

$9.90 \times 10^{-6}$

$8.2 \times 10^{-6}$
$1.00 \times 10^{-5}$

$2.16 \times 105$

0
$4.52 \times 1$
$1.25 \times 10$

n.d.

not connected

$3.8 \pm 1.4$
$5.2+22$
$39 \pm 1.1$

$3.2 \pm 2.2$
$3.9 \pm 1.1$

$4.23 \times 10^{-16}$
$3.44 \times 10^{-13}$
$1.41 \times 10^{-12}$

$1.41 \times 10^{12}$

$3.23 \times 10^{12}$

$\begin{array}{ll}1.01 \times 10^{-5} & 1.16 \times 10^{-5} \\ 1.26 \times 10^{-5} & 1.29 \times 10^{5} \\ 121 \times 10^{5} & 1.16 \times 10^{5}\end{array}$

$8.00 \times 10^{-6}$

$8.2 \times 10^{-6}$

$4.0 \pm 2.3$

$4.1 \pm 3.4$

$\begin{array}{llll}8.2 \times 10^{-6} & 1.29 \times 10^{-5} & 3.74 \times 10^{2} & 1.68 \pm 0.20 \\ 8.2 \times 10^{-6} & 1.42 \times 10^{-5} & 5.8610^{2} & 1.99 \pm 051 \\ 82 \times 10^{-0} & 1.29 \times 10^{5} & 151 \times 10^{-} & 1.72 \pm 051\end{array}$

$\begin{array}{llll}8.2 \times 10^{-6} & 1.29 \times 10^{-5} & 1.51 \times 10^{3} & 1.72 \pm 0.5 \\ 82 \times 10^{-6} & 1.2 \times 10 \times 10^{5} & 1.75 \times 10^{8} & 1.51 \pm 0\end{array}$

$\begin{array}{llll}8.2 \times 10^{-6} & 2.16 \times 10^{-5} & 2.40 \times 10^{4} & 1.39 \pm 0.25 \\ 82 \times 10^{6} & 1.23 \times 10^{5} & 424 \times 10^{4} & 1.33 \pm 0.22\end{array}$

$8.2 \times 10^{-6}$

$8.2 \times 10^{-6}$

$8.2 \times 10^{-6}$

$2.16 \times 10^{-5}$
$1.83 \times 10^{-5}$

$3.8 \pm 1.0$

$3.6 \pm 1.4$
$40+24$
$42 \pm 3$.

$4.0 \pm 2.4$
$4.2 \pm 3.1$
$4.4 \pm 38$

$2.16 \times 10^{-5} \quad 5.73 \times 10^{4} \quad 1.29 \pm 0.20$

$2.83 \times 10^{-5} \quad 9.43 \times 10^{4} \quad 1.21 \pm 0.13$

$\begin{array}{llll}8.2 \times 10^{-6} & 2.89 \times 10^{-5} & 1.05 \times 10^{5} & 1.13 \pm 0.07 \\ 8.2 \times 10^{-6} & 3.56 \times 10^{-5} & 8.68 \times 10^{4} & 1.12 \pm 0.06 \\ 82 \times 10^{-1} & 5.04 \times 10^{5} & 800 \times 10^{4} & 1.10 \pm 005\end{array}$

$\begin{array}{lllll}8.2 \times 10^{-6} & 5.04 \times 10^{-5} & 8.46 \times 10^{4} & 1.10 \pm 0.05 \\ 82 \times 10^{-5} & 3.92 \times 10^{5} & 8.00 \times 10^{-1} & 1.0 \pm 0.0\end{array}$

$\begin{array}{ll}1.36 \times 10^{-5} & 1.29 \times 10^{-5} \\ 1.46 \times 10^{-5} & 1.29 \times 10^{5} \\ 1.40 \times 15 & 1.5 \times 10^{5}\end{array}$

$140 \times 10^{-5}-1.25 \times 10^{5}-9.50 \times 10^{5}-1.00 \times 10^{5}-9.00 \times 10^{6}$

$1.56 \times 10^{-5} \quad 1.42 \times 10^{-5} \quad 9.70 \times 10^{-5}$

$\begin{array}{lll}1.65 \times 10^{-5} & 1.42 \times 10^{-5} & 9.87 \times 10^{\circ} \\ 2.14 \times 10^{-5} & 1.73 \times 10^{-5} & 1.06 \times 10^{5}\end{array}$

$100 \times 10^{-5}$

$\begin{array}{lll}3.92 \times 10^{-5} & 8.00 \times 10^{4} & 1.10 \pm 0.05 \\ 4.51 \times 10^{-5} & 5.17 \times 10^{4} & 1.10=0.04\end{array}$

$4.4 \pm 3.8$
$5.2 \pm 7.8$

$5.5 \pm 12.3$

$5.2 \pm 7.1$

$6.3 \pm 12.7$

$7.7 \pm 12.7$

$2.98 \times 10^{11}$

$1.35 \times 10^{-14}$

$1.45 \times 10^{-14}$
$3.94 \times 10^{-14}$

$1.50 \times 10^{-13}$
$2.99 \times 10^{-13}$

$4.04 \times 10^{-13}$

$9.82 \times 10^{13}$

$3.23 \times 10^{-12}$

$4.46 \times 10^{12}$

$5.60 \times 10^{12}$

$9.56 \times 10^{-12}$

$\begin{array}{lll}1.14 \times 10^{-5} & 1.16 \times 10^{-5}-7.9 \times 10^{-6} \\ 1.36 \times 10^{5} & 1.29 \times 10^{5} & 90 \times 10^{5}\end{array}$

$\begin{array}{llll}8.2 \times 10^{-6} & 1.64 \times 10^{-5} & 6.09 \times 10^{3} & 1.48 \pm 0.31 \\ 82 \times 10^{-6} & 352 \times 10^{-5} & 124 \times 10^{5} & 1.09 \pm 006\end{array}$

$4.5 \pm 6.8$

$\begin{array}{lll}3.52 \times 10^{-5} & 1.24 \times 10^{5} & 1.09 \pm 0.06 \\ 6.96 \times 10^{-5} & 3.25 \times 10^{4} & 1.09 \pm 0.03\end{array}$

$4.5 \pm 6.8$
$4.7 \pm 4.6$

no results

$5.47 \times 10^{-12}$

$\begin{array}{ll}4.7 \pm \pm .0 \pm 11.5 & 5.22 \times 10^{-11} \\ & \end{array}$

Vesiculation conditions

'Volume used for all analyses

Wosties and connected porosity in these columns were determined on the volumes used for permeability measurements. See text for discussion Bubble number density (BND)

SPore throat density (PTD) divided by bubble number density

${ }^{6} \beta$, or connective density, is defined in Equation 1

Time-temperature histories and heating rates for experiments EF-8a, DRB2012-2a, and DRB2012-6e were extracted from time-temperature charts recorded during the experiments.

Time is in seconds after heating above $600^{\circ} \mathrm{C}$. Precision of time is within $\sim 2 \mathrm{~s}$ and of temperature within $10^{\circ} \mathrm{C}$, athough temperature fluctuations as great as $\pm 50^{\circ} \mathrm{C}$ were sometimes observed (DRB2012-6e-10)
o Supersaturation pressure at start of experiment calculated using Papale et al (2006) as implemented on hitp://melts. ofm-research.org/CORBA_CTserver/Papale/Papale.php 
Table 2: Results of bubble growth experiments

\begin{tabular}{|c|c|c|c|c|c|}
\hline Sample & $\begin{array}{c}\text { Volume }{ }^{1} \\
\left(\mathrm{~mm}^{3}\right)\end{array}$ & Porosity ${ }^{2}$ & $\begin{array}{c}\text { Connected } \\
\text { Porosity }^{2}\end{array}$ & $\operatorname{BND}^{3}\left(\mathrm{~mm}^{-3}\right)$ & $\operatorname{PTD}^{4}\left(\mathrm{~mm}^{-3}\right)$ \\
\hline
\end{tabular}

Basalt, $3 \% \mathrm{H}_{2} \mathrm{O}, \Delta P=68 \mathrm{MPa}^{8}$

$\begin{array}{cccccc}\text { DRB2012-07a-2 } & 0.0989 & 0.52 & 0.52 & 4309 & 6403 \\ \text { DRB2012-07a-3 } & 0.0989 & 0.50 & 0.50 & 5290 & 8092 \\ \text { DRB2012-7f-10 } & 0.1931 & 0.55 & 0.55 & 3009 & 7706 \\ \text { DRB2012-7c-f } & 0.0989 & 0.73 & 0.73 & 19228 & 72157\end{array}$

Trachyandesite, $2.8 \% \mathrm{H}_{2} \mathrm{O}, \Delta P=35 \mathrm{MPa}$

$\begin{array}{llllcc}\text { EFJ-8a-06 } & 0.0506 & 0.09 & 0.04 & 20 & 0 \\ \text { EFJ-8a-07 } & 0.0506 & 0.35 & 0.33 & 8989 & 13493 \\ \text { EFJ-8a-08 } & 0.0506 & 0.61 & 0.61 & 6934 & 19419 \\ \text { EFJ-8a-09 } & 0.0506 & 0.64 & 0.64 & 2331 & 4741\end{array}$

Andesite, $5 \% \mathrm{H}_{2} \mathrm{O}, \Delta P=168 \mathrm{MPa}$

$\begin{array}{llllcc}\text { DRB2012-2a-01 } & 0.0989 & 0.17 & 0.15 & 233 & 597 \\ \text { DRB2012-2a-08 } & 0.0989 & 0.28 & 0.28 & 506 & 1082 \\ \text { DRB2012-2a-09 } & 0.0989 & 0.28 & 0.28 & 273 & 415 \\ \text { DRB2012-2a-10 } & 0.0989 & 0.33 & 0.33 & 3216 & 4956 \\ \text { DRB2012-2a-11 } & 0.0989 & 0.41 & 0.41 & 26915 & 50876 \\ \text { DRB2012-2a-12 } & 0.0989 & 0.46 & 0.46 & 41601 & 83971 \\ \text { DRB2012-2a-13 } & 0.0989 & 0.51 & 0.51 & 46112 & 103381 \\ \text { DRB2012-2a-14 } & 0.0989 & 0.59 & 0.59 & 51453 & 145761 \\ \text { DRB2012-2a-15 } & 0.0989 & 0.71 & 0.71 & 31841 & 136324 \\ \text { DRB2012-2a-16 } & 0.0989 & 0.73 & 0.74 & 26035 & 112797 \\ \text { DRB2012-2a-17 } & 0.0989 & 0.79 & 0.79 & 25003 & 109611 \\ \text { DRB2012-2a-18 } & 0.0989 & 0.79 & 0.79 & 22768 & 102774 \\ \text { DRB2012-2a-19 } & 0.0989 & 0.80 & 0.80 & 15131 & 66847\end{array}$


Dacite, $3 \% \mathrm{H}_{2} \mathrm{O}, \Delta P=74 \mathrm{MPa}$

\begin{tabular}{|c|c|c|c|c|c|}
\hline DRB2012-6e-7 & 0.1931 & 0.37 & 0.37 & 4806 & 10891 \\
\hline DRB2012-6e-8 & 0.0989 & 0.79 & 0.79 & 23344 & 147612 \\
\hline DRB2012-6e-9 & 0.0989 & 0.87 & 0.87 & 6160 & 38688 \\
\hline DRB2012-6e-10 & 0.0989 & 0.84 & 0.84 & 4015 & 17205 \\
\hline
\end{tabular}

${ }^{1}$ Volume used for all analyses

${ }^{2}$ Porosities and connected porosity in these columns were determined on the volumes used for permeabil ${ }^{3}$ Bubble number density (BND)

${ }^{4}$ Pore throat density (PTD)

${ }^{5}$ Pore throat density (PTD) divided by bubble number density

${ }^{6} \beta$, or connective density, is defined in Equation 1

${ }^{7}$ Time-temperature histories and heating rates for experiments EFJ-8a, DRB2012-2a, and DRB2012-6e $\mathrm{n}$ Time is in seconds after heating above $600^{\circ} \mathrm{C}$. Precision of time is within $\sim 2 \mathrm{~s}$ and of temperature with

${ }^{8}$ Supersaturation pressure at start of experiment calculated using Papale et al. (2006) as implemented on 


$\begin{array}{llllllll}1.486 & 2.24 \times 10^{-5} & 1.92 \times 10^{-5} & 1.08 \times 10^{-5} & 8.2 \times 10^{-6} & 5.36 \times 10^{-5} & 2.10 \times 10^{3} & 1.33 \pm 0.10 \\ 1.530 & 2.10 \times 10^{-5} & 1.73 \times 10^{-5} & 1.01 \times 10^{-5} & 8.2 \times 10^{-6} & 5.36 \times 10^{-5} & 2.81 \times 10^{3} & 1.34 \pm 0.10 \\ 2.561 & 2.67 \times 10^{-5} & 1.91 \times 10^{-5} & 1.11 \times 10^{-5} & 8.2 \times 10^{-6} & 9.89 \times 10^{-5} & 4.70 \times 10^{3} & 1.20 \pm 0.07 \\ 3.753 & 2.45 \times 10^{-5} & 2.31 \times 10^{-5} & 1.01 \times 10^{-5} & 8.2 \times 10^{-6} & 3.37 \times 10^{-5} & 5.29 \times 10^{4} & 1.15 \pm 0.08\end{array}$

$0.000 \quad 8.20 \times 10^{-6}$

$8.2 \times 10^{-6}$ $3.56 \times 10^{-5}$ $1.03 \times 10^{-5}$ 
lity measurements. See text for discussion

ıere extracted from time-temperature charts recorded during the experiments.

in $\sim 10^{\circ} \mathrm{C}$, although temperature fluctuations as great as $\pm 50^{\circ} \mathrm{C}$ were sometimes observed (DRB2012-6e-10)

। http://melts.ofm-research.org/CORBA_CTserver/Papale/Papale.php 


\section{Permeability}

$\begin{array}{lll}\text { Coordination \# } & \left(\mathrm{m}^{2}\right) & \text { Vesiculation conditions }^{7}\end{array}$

$\begin{array}{ll}4.4 \pm 6.8 & 1.28 \times 10^{-12} \\ 4.3 \pm 7.1 & 3.49 \times 10^{-13} \\ 5.2 \pm 6.8 & 1.50 \times 10^{-11} \\ 6.8 \pm 6.2 & 2.92 \times 10^{-11}\end{array}$

not connected $\quad 4.23 \times 10^{-16}$ $3.8 \pm 1.4 \quad 3.44 \times 10^{-13}$ $5.2 \pm 2.2 \quad 1.41 \times 10^{-12}$ $3.9 \pm 1.1 \quad 3.23 \times 10^{-12}$

$\begin{array}{cl}4.0 \pm 2.3 & 2.98 \times 10^{-15} \\ 4.1 \pm 3.4 & 1.35 \times 10^{-14} \\ 3.8 \pm 1.0 & 1.45 \times 10^{-14} \\ 3.6 \pm 1.4 & 3.94 \times 10^{-14} \\ 4.0 \pm 2.4 & 1.50 \times 10^{-13} \\ 4.2 \pm 3.1 & 2.99 \times 10^{-13} \\ 4.4 \pm 3.8 & 4.04 \times 10^{-13} \\ 5.2 \pm 7.8 & 9.82 \times 10^{-13} \\ 5.5 \pm 12.3 & 3.23 \times 10^{-12} \\ 5.2 \pm 7.1 & 4.46 \times 10^{-12} \\ 6.3 \pm 12.7 & 5.60 \times 10^{-12} \\ 6.8 \pm 12.7 & 5.76 \times 10^{-12} \\ 7.7 \pm 12.7 & 9.56 \times 10^{-12}\end{array}$

Manual heating to $970{ }^{\circ} \mathrm{C}$, hold $75 \mathrm{~s}, 1^{\text {st }}$ bubbles: $616{ }^{\circ} \mathrm{C}$ Re-heating of DRB 2012-07a-2, quenched $1^{\circ} \mathrm{C} \mathrm{s}^{-1}$ from 600 to 1150 , hold $150 \mathrm{~s}, 1$ st vesiculation: $740^{\circ} \mathrm{C}$ $6{ }^{\circ} \mathrm{C} \mathrm{s}^{-1}$ from 600 to 1150 , hold $40 \mathrm{~s}$, 1 st vesiculation: $880^{\circ} \mathrm{C}$

$0.8^{\circ} \mathrm{C} \mathrm{s}^{-1}$ from 600 to 950 , hold $150 \mathrm{~s}, 1$ st vesiculation: $720^{\circ} \mathrm{C}$ $325 \mathrm{~s}, 860{ }^{\circ} \mathrm{C}$ ( 1 bubble found)
$413 \mathrm{~s}, 930{ }^{\circ} \mathrm{C}$
$380 \mathrm{~s}, 980{ }^{\circ} \mathrm{C}$
$471 \mathrm{~s}, 950{ }^{\circ} \mathrm{C}$

$5{ }^{\circ} \mathrm{C} \mathrm{s}^{-1}$ from 600 to 1100 , hold $15 \mathrm{~s}$, 1 st vesiculation: $900^{\circ} \mathrm{C}$ $60 \mathrm{~s}, 900{ }^{\circ} \mathrm{C}$ $77 \mathrm{~s}, 984^{\circ} \mathrm{C}$ $79 \mathrm{~s}, 994^{\circ} \mathrm{C}$ $81 \mathrm{~s}, 1005{ }^{\circ} \mathrm{C}$ $83 \mathrm{~s}, 1016{ }^{\circ} \mathrm{C}$ $85 \mathrm{~s}, 1026{ }^{\circ} \mathrm{C}$ $87 \mathrm{~s}, 1037{ }^{\circ} \mathrm{C}$ $89 \mathrm{~s}, 1047^{\circ} \mathrm{C}$ $92 \mathrm{~s}, 1057{ }^{\circ} \mathrm{C}$ $94 \mathrm{~s}, 1168{ }^{\circ} \mathrm{C}$ $96 \mathrm{~s}, 1179{ }^{\circ} \mathrm{C}$ $98 \mathrm{~s}, 1089{ }^{\circ} \mathrm{C}$ $100 \mathrm{~s}, 1100{ }^{\circ} \mathrm{C}$ 
$1{ }^{\circ} \mathrm{C} \mathrm{s}^{-1}$ from 600 to 1150 , hold $150 \mathrm{~s}, 1$ st vesiculation: $790^{\circ} \mathrm{C}$

$\begin{array}{llc}4.5 \pm 6.8 & \text { no results } & 373 \mathrm{~s}, 980^{\circ} \mathrm{C} \\ 4.7 \pm 4.6 & 5.47 \times 10^{-12} & 446 \mathrm{~s}, 1050^{\circ} \mathrm{C} \\ 4.7 \pm 5.6 & 5.29 \times 10^{-11} & 504 \mathrm{~s}, 1110^{\circ} \mathrm{C} \\ 7.0 \pm 11.5 & 3.22 \times 10^{-11} & 585 \mathrm{~s}, \sim 1200{ }^{\circ} \mathrm{C}\end{array}$


Click here to access/download Supplementary Material SupplementaryMovie_DRB2012_2a_Rendering.gif 
Click here to access/download Supplementary Material SupplementaryDataPoreAndThroatSizes.xls 
Click here to access/download Supplementary Material SupplementaryFigure1.eps 